\title{
Pressure and Flow Residence Time Effects on Soot Properties in Counterflow Non-Premixed Hydrocarbon-Air Flames
}

A Dissertation
Presented to
the Faculty of the School of Engineering and Applied Science
University of Virginia

\author{
In Partial Fulfillment \\ of the Requirements for the Degree of \\ Doctor of Philosophy in Mechanical and Aerospace Engineering
}

\author{
by \\ Brendyn G. Sarnacki
}

May 2014 


\section{Approval Sheet}

This dissertation is submitted in partial fulfillment of the requirements for the degree of Doctor of Philosophy in Mechanical and Aerospace Engineering

Brendyn G. Sarnacki

This dissertation has been read and approved by the Examining Committee:

Prof. Eric Loth, Committee Chairman

Prof. Harsha K. Chelliah, Dissertation Advisor

Dr. Roland H. Krauss

Prof. Gabriel Laufer

Prof. Charles A. Sackett

Accepted for the School of Engineering and Applied Science:

Dean, School of Engineering and Applied Science

May 2014 


\section{Abstract}

Numerous studies have reported the adverse health, environmental, and climatic effects of aerosol or soot particulate emissions from the combustion of hydrocarbon fuels in boilers, furnaces, gas turbines and other internal combustion engines. Considering the significant dependence that our modern society places on hydrocarbon fuels, it is of ethical interest to reduce or mitigate the resulting pollutants. With advanced laser based diagnostic techniques under development, the potential for future regulation on particulate emissions provides further motivation. While production of some pollutant species is well understood, knowledge of soot particulate nucleation and growth remains in its infancy. Precise synthesis of flame generated carbon nanoparticles may also prove useful as an industrial and technical commodity to increase efficiency and reduce cost for a variety of applications. One of the most elementary and important effects on soot formation and growth relevant to modern combustion engines is that of pressure. Utilizing the simple laminar, steady counterflow burner configuration, the goal of this work is to investigate the effect of elevated pressure on the soot nucleation, growth, and oxidation mechanisms of hydrocarbon combustion over a wide range of flow residence times.

An absolute irradiance calibrated two-color time resolved Laser Induced Incandescence (LII) technique was developed and utilized to collect quantitative soot incandescence data for determination of soot particle temperature, primary particle size, soot volume fraction, and number density. The approach requires a comprehensive LII nano-scale heat transfer model with coupled extinction and elastic light scattering submodels. Thermophoretic soot 
sampling and Transmission Electron Microscopy (TEM) analysis were conducted to provide further insight into soot structure and morphology. Particle Image Velocimetry (PIV) was also employed to quantify flow residence time. All sources of uncertainty from both measured experimental variables and assumed heat transfer model variables were considered and included in a detailed uncertainty analysis to determine total uncertainties on all LII extracted soot quantities. The uncertainty analysis of PIV measurements was also presented. The resulting total standard uncertainties of soot volume fraction, primary particle size, and soot number density are reported. Several improvements to the LII procedure are presented. The results indicate that uncertainty associated with the local gas temperature, soot absorption function, and thermal accommodation are the dominant variables dictating uncertainty on all LII extracted quantities. Experimental repeatability uncertainty was found significantly lower than the combined total standard measurement uncertainty for soot volume fraction and primary particle diameter indicating additional research into key heat transfer model parameters is still needed to reduce the overall uncertainty of LII measurements.

The results of TEM analysis on thermophoretically sampled soot are presented. An extensive analysis of number density, soot volume fraction, and primary particle size is conducted. Particle nucleation is identified as the driver of total soot loading in the counterflow configuration and exhibits a clear pressure and temperature dependency. The results indicate that increased combustion pressure should be targeted to reduce total soot loading while allowing for increased combustion temperatures before soot particle nucleation is initiated. From thermodynamic first principles, increased pressure and temperature are directly related to improved engine efficiency and energy density. The finding is encouraging for practical application in industry. 


\section{Acknowledgements}

To my friends and loved ones for their support and guidance. My sincerest gratitude to my advisor Dr. Harsha Chelliah and committee members Dr. Eric Loth, Dr. Roland Krauss, Dr. Gabriel Laufer, and Dr. Charles Sackett for their sage advice. This work was supported by Rolls-Royce and the Commonwealth Center for Aerospace Propulsion Systems, with Dr. MS Anand at Rolls-Royce as the technical monitor. 


\section{Contents}

List of Figures $\quad$ iv

List of Tables

1 Introduction 1

1.1 Motivation . . . . . . . . . . . . . . . . . . 1

1.2 Selection of Equipment . . . . . . . . . . . . . . 4

1.2.1 Selection of Burner Configuration . . . . . . . . . . . . 4

1.2.2 Selection of Measurement System . . . . . . . . . . . . . . 6

1.3 Historical Background . . . . . . . . . . . . . . . . 9

1.3.1 A Brief History of Soot Research . . . . . . . . . . . . . . 9

1.3.2 A Brief History of Laser Induced Incandescence . . . . . . . . . . . 12

1.4 Dissertation Objectives . . . . . . . . . . . . . . . . . . . . . . . . . . . . . . . . . 14

1.5 Dissertation Outline . . . . . . . . . . . . . . . . . . . . . . . . 14

2 Research Design and Methodology 16

2.1 General Experimental Methodology . . . . . . . . . . . . . . . . . 16

2.2 Supporting Numerical Results . . . . . . . . . . . . . . . . 20

2.3 Laser Induced Incandescence Methodology . . . . . . . . . . . . . . . 21

2.3.1 Absolute Irradiance Calibration of ICCD Camera . . . . . . . . . . 21

2.3.2 Beam Profile and Fluence Calibration . . . . . . . . . . . . . 23

2.3.3 Particle Temperature . . . . . . . . . . . . . . . . 24

2.3.4 Primary Particle Size . . . . . . . . . . . . . 26

2.3.5 Soot Volume Fraction . . . . . . . . . . . . . . . 27

2.3.6 Number Density . . . . . . . . . . . . . . . . . . 28

2.3.7 Heat Transfer Model . . . . . . . . . . . . . . . . . . . . . . 29

2.3.8 Heat Transfer Model Validation . . . . . . . . . . . . . . . . . . . . 34

2.4 Elastic Light Scattering Aggregate Submodel . . . . . . . . . . . . . . 37

2.5 Extinction Correction . . . . . . . . . . . . . . . . . 41

2.6 Thermophoretic Sampling Probe . . . . . . . . . . . . . 45

3 Uncertainty Analysis

3.1 LII Uncertainty . . . . . . . . . . . . . . . . . . . . . . . 47

3.1.1 Detailed LII Model Parameter Uncertainty _ . . . . . . . . . . . . . . . 47

3.1.2 Uncertainty Analysis of LII Measurements . . . . . . . . . . . 56 
3.1.3 Mixture Averaged Thermal Accommodation Coefficient, $\alpha_{T}$. . . 58

3.1.4 Estimation of Spatially Varying Absorption Function . . . . . . . . . . 66

3.1.5 Final LII Measurement Uncertainty . . . . . . . . . . . . . . . 71

3.1 .6 Repeatability Uncertainty . . . . . . . . . . . . . . 73

3.1.7 Local Gas Properties and Other Uncertainties . . . . . . . . . . . . 76

3.2 Particle Image Velocimetry Uncertainty . . . . . . . . . . . . . . . 77

3.2.1 Measurement Uncertainty . . . . . . . . . . . . . . . . . 77

3.2.2 Uncertainty of the Regression Model . . . . . . . . . . . . . . . 80

3.2.3 Repeatability . . . . . . . . . . . . . . . 81

3.2 .4 Total Uncertainty . . . . . . . . . . . . . . . . 82

4 Experimental Results $\quad \mathbf{8 3}$

4.1 Transmission Electron Microscopy Results . . . . . . . . . . . . . 83

4.1.1 Fractal Aggregate Dimensions . . . . . . . . . . . . . . . . 84

4.1.2 Primary Particle Diameter and Aggregate Size Distributions . . . . 87

4.1.3 Soot Particle Morphology . . . . . . . . . . . . . . . . . 88

4.2 Soot Particle Temperature . . . . . . . . . . . . . . . . . . 91

4.3 Soot Particle Number Density . . . . . . . . . . . . . . . . . . . . . . . . . . . . . . . . . . .

4.4 Soot Volume Fraction . . . . . . . . . . . . . . . . . . . . . . . . . . . . . . . . . . . . . . . . .

4.5 Primary Soot Particle Diameter . . . . . . . . . . . . . . . . . . . 114

4.6 Elastic Light Scattering Results . . . . . . . . . . . . . . . . . . . 120

4.7 Sooting Limits . . . . . . . . . . . . . . . . . . 123

5 Summary and Recommendations $\quad 128$

$\begin{array}{ll}\text { Bibliography } & 133\end{array}$ 


\section{List of Figures}

1.1 World total energy consumption. . . . . . . . . . . . . . . 1

1.2 A conceptual representation of soot formation. . . . . . . . . . . . 3

1.3 Illustration of different burner configurations. . . . . . . . . . 5

2.1 High pressure counterflow burner with assembled LII system. . . . . . . . . 17

2.2 Counterflow burner nozzle assembly. . . . . . . . . . . . . . . . . 18

2.3 Schematic and general layout of absolute irradiance calibrated LII and PIV systems. . . . . . . . . . . . . . . . . . . . . . . 19

2.4 Gamma Scientific RS-10D calibrated light source used for imaging irradiance onto intensified camera. . . . . . . . . . . . . . . . . . . . . 22

2.5 Profile of laser sheet used in LII studies. . . . . . . . . . . . . . . . . 23

2.6 Fluence calibrated laser beam sheet width profiles for laser settings from low to high fluence LII. . . . . . . . . . . . . . . . . . . . . . . . . . 24

2.7 Example of iterative least-squares fitting of model and experimentally measured particle temperature decays. . . . . . . . . . . . . . . . 27

2.8 Illustration of the heat transfer mechanisms involved in the soot particle energy balance. . . . . . . . . . . . . . . . . . . . 29

2.9 Nusselt numbers calculated from several heat conduction models. . . . . . 35

2.10 Comparison of modeled temperature and LII signal decay from the semiconstrained independent models. . . . . . . . . . . . . . 36

2.11 Flame geometry for calculating laser and signal extinction. . . . . . . . . . 42

2.12 Mechanical device and electrical circuit constructed for thermophoretic soot sampling. . . . . . . . . . . . . . . . . . 45

3.1 Profiles of temperature and major species mole fractions for samples at $4.065 \mathrm{~atm}$ and $14 \mathrm{~atm}$. . . . . . . . . . . . . . . . . 60

3.2 LII fitting results of spatially varying absorption function $E(m)$ for a sample at $1 \mathrm{~atm} . \ldots \ldots \ldots$. . . . . . . . . . . . . 70

3.3 A comparison of LII determined normalized volume fraction with assumptions of constant and spatially varying absorption function $E(m) \ldots \ldots$. . 71

3.4 Soot volume fraction repeatability study. . . . . . . . . . . . . . 74

3.5 Soot primary particle diameter repeatability study. . . . . . . . . . . . 75

3.6 Soot number density repeatability study. . . . . . . . . . . . . 76

3.7 Example of a reacting counterflow axial velocity profile across the burner axial centerline. . . . . . . . . . . . . . 78 
4.1 TEM aggregate images for a sample at a pressure of $8.45 \mathrm{~atm} . \quad \ldots . . . . \quad 84$

4.2 Number of primary particles per aggregate $N_{p}$ as a function of maximum aggregate length and primary particle diameter $L / d_{p}$. . . . . . . . . . . 86

4.3 Histogram of normalized primary particle diameter $d_{p}$ and number of primary soot particles per aggregate $N_{p} \ldots \ldots \ldots$. . . . . . 88

4.4 High resolution TEM images of soot structure for a sample at $8.45 \mathrm{~atm}$. . . 90

4.5 Temperature profiles for several local strain rates at $4.065 \mathrm{~atm}$ and $14 \mathrm{~atm}$. . 92

4.6 Normalized soot particle number density for several local strain rates at

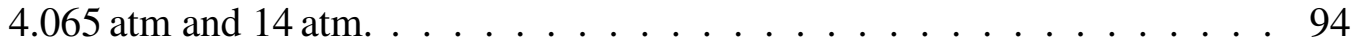

4.7 LII measured normalized maximum soot particle number density as a function of 1/RT over a range of pressures from 1 to $30 \mathrm{~atm}$ and a range of strain rates. . . . . . . . . . . . . . . . . . . . 98

4.8 Normalized soot particle number density as a function of particle residence time for several local strain rates at $4.065 \mathrm{~atm}$ and $14 \mathrm{~atm}$. . . . . . . . . . . 101

4.9 Normalized soot particle number density growth rates as a function of particle residence time for several local strain rates at $4.065 \mathrm{~atm}$ and $14 \mathrm{~atm} . ~ . ~ 103$

4.10 Normalized soot volume fraction for several local strain rates at $4.065 \mathrm{~atm}$ and 14 atm. . . . . . . . . . . . . . . . . . . . 107

4.11 LII measured normalized maximum soot particle number density as a function of volume fraction over a range of pressures from 1 to $30 \mathrm{~atm}$ and a range of strain rates. . . . . . . . . . . . . . . . . . 108

4.12 Normalized soot volume fraction as a function of particle residence time for several local strain rates at $4.065 \mathrm{~atm}$ and $14 \mathrm{~atm}$. . . . . . . . . . . . . 110

4.13 Normalized soot volume fraction growth rates as a function of particle residence time for several local strain rates at $4.065 \mathrm{~atm}$ and $14 \mathrm{~atm}$. . . . . . . 112

4.14 Normalized soot primary particle diameter for several local strain rates at $4.065 \mathrm{~atm}$ and $14 \mathrm{~atm}$. . . . . . . . . . . . . . . . . 116

4.15 Normalized primary soot particle diameter as a function of particle residence time for several local strain rates at 4.065 atm and 14 atm. . . . . . 118

4.16 Normalized primary soot particle volumetric growth rates as a function of particle residence time for several local strain rates at $4.065 \mathrm{~atm}$ and $14 \mathrm{~atm} .119$

4.17 Normalized primary soot particle volumetric growth rates per surface area as a function of particle residence time for several local strain rates at $4.065 \mathrm{~atm}$ and $14 \mathrm{~atm}$. . . . . . . . . . . . . . . . . . . . 120

4.18 LII extracted measurements of particle temperature and normalized soot volume fraction, primary particle diameter, and number density for a sample at 8.45 atm. . . . . . . . . . . . . . . . . . . . 122

4.19 LII and ELS extracted measurements of the normalized number of primary particles per aggregate, soot volume fraction, primary particle diameter, and number density for a sample at 8.45 atm. . . . . . . . . . . . . 123

4.20 LII measured normalized maximum soot volume fraction as a function of local strain rate over a range of pressures from 1 to $30 \mathrm{~atm}$. . . . . . . . . 125 


\section{List of Tables}

2.1 Parameters for heat transfer model . . . . . . . . . . . . . . . . . . 34

3.1 List of parameters involved in Laser Induced Incandescent analysis and their associated uncertainties. Experimental and model variables/parameters are listed at the top and the bottom, respectively. . . . . . . . . . . . . 57

3.2 Uncertainties in Laser Induced Incandescence extracted variables. . . . . . 58

3.3 Relevant species thermal accommodation coefficients $\left(\alpha_{T, i}\right)$ and documented uncertainties from literature. . . . . . . . . . . . . . . . . 64

3.4 List of top 10 most important species thermal accommodation coefficients $\left(\alpha_{T, i}\right)$ and uncertainty. . . . . . . . . . . . . . . . 65

3.5 Revised uncertainties in Laser Induced Incandescence extracted variables. . 72

3.6 Estimated PIV measurement uncertainty. . . . . . . . . . . . . . . . 79 


\section{Nomenclature}

$\alpha$
$\alpha_{M}$
$\alpha_{T, i}$
$\beta_{0}$
$\beta_{1}$
$\beta_{2}$
$\Delta \beta_{1, k}$
$\Delta \beta_{2, k}$
$\Delta a_{l, k}$
$\Delta a_{l, \text { reg }}$
$\Delta a_{l, \text { rep }}$
$\Delta a_{l, \text { tot }}$
$\Delta a_{l}$
$\Delta v_{P I V}$
$\Delta v_{\text {slip }}$
$\Delta v_{\text {tot }}$
$\Delta x_{i}$
$\Delta y_{i, j}$
$\delta$
$\Delta \beta_{0}$
$\Delta \beta_{1}$
$\dot{Q}_{a b s o r p t i o n}$
$\dot{Q}_{\text {conduction }}$
$\dot{Q}_{C}$
$\dot{Q}_{F M}$
$\dot{Q}_{\text {radiation }}$
$\dot{Q}_{\text {sublimation }}$
$\epsilon_{\lambda}$
$\eta_{i}$
$d \sigma_{N_{2}}$
$\frac{d M}{d t}$
$\gamma$
$\gamma_{h}$
$\kappa$
$\kappa_{h}$

Gas mixture thermal diffusivity.

Mass accommodation coefficient.

Individual species thermal accommodation coefficient.

Zeroth order regression coefficient.

First order regression coefficient.

Second order regression coefficient.

Uncertainty of first order regression coefficient of $k$-th experiment.

Uncertainty of second order regression coefficient of $k$-th experiment.

Local strain rate uncertainty of $k$-th experiment.

Local strain rate uncertainty from regression.

Local strain rate uncertainty from repeatability over $k$ experiments.

Total local strain rate uncertainty.

Local strain rate uncertainty.

PIV measured velocity uncertainty.

Particle slip velocity uncertainty.

Total velocity uncertainty.

Length of travel through the $i$ th pixel in the $x$-dimension.

Length of travel through the $j$-th pixel in the $y$-dimension.

Reaction layer thickness.

Uncertainty in zeroth order regression coefficient.

Uncertainty in first order regression coefficient.

Rate of pulsed laser energy absorbed by soot particle.

Rate of energy lost from soot particle by conduction.

Continuum heat conduction rate.

Free molecular heat conduction rate.

Rate of energy lost from soot particle by radiation.

Rate of energy lost from soot particle by sublimation.

Soot emissivity.

CCD camera calibration factor.

Differential scattering cross section of nitrogen.

Mass loss rate of carbon from soot particle.

Local gas species average heat capacity ratio.

Harmonic mean specific heat capacity ratio.

Local gas species average thermal conductivity.

Harmonic mean thermal conductivity. 


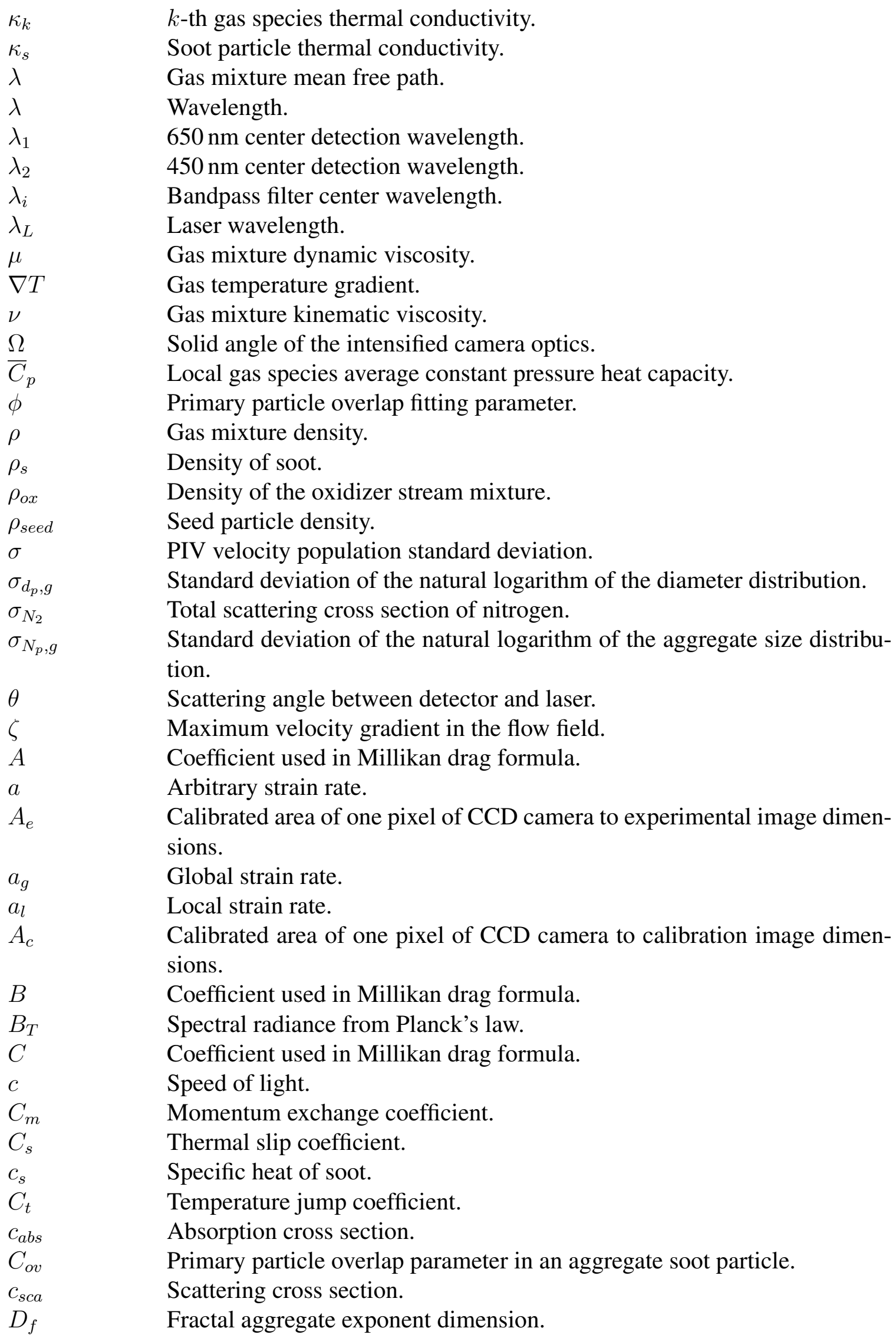


$d_{s}$

$D_{\text {eff }}$

$D_{f L}$

$d_{p}$

$d v_{z} / d z$

$E(m)$

$E_{a}$

$f$

$F_{0}$

$F_{T}$

$F_{\text {ext }}$

$f_{v}$

$h$

$I_{c}\left(\lambda_{i}\right)$

$I_{m}\left(\lambda_{i}\right)$

$I_{\text {inc }}$

$I_{L I I, 0}(i)$

$I_{L I I, \text { ext }}(i)$

$I_{L I I, n}\left(\lambda_{i}\right)$

$I_{L I I, p}\left(\lambda_{i}\right)$

$I_{L I I}$

$I_{L S}$

$I_{m, p}\left(\lambda_{i}\right)$

$I_{\text {sca }, 0}(i)$

$I_{s c a, c}$

$I_{\text {sca,ext }}(i)$

$I_{\text {sca }}$

$k_{B}$

$k_{f}$

$k_{L}$

$L$

$m$

$N$

$N_{p}$

$N_{a g}$

$N_{N_{2}}$

$p_{0}$

$p_{v}$

$p_{\text {ref }}$

$q$

$q(t) / q_{1}$
Seed particle diameter.

Effective particle diameter due to surface shielding for conduction heat loss.

Projected aggregate slope parameter.

Primary particle diameter.

Axial velocity gradient.

Absorption function of soot.

Activation energy of a global reaction.

Eucken formula $f=(9 \gamma-5) / 4$.

Laser fluence.

Thermophoretic force acting on soot particles from the counterflow flame temperature gradient.

Laser fluence after extinction through soot layer.

Soot volume fraction.

Planck constant.

Absolute spectral irradiance at a bandpass filter center wavelength.

Modeled incandescent signal at a center detection wavelength.

Incident laser intensity.

Measured LII signal before extinction correction.

LII signal corrected for extinction through soot layer.

Incandescent signal after peak signal at a center detection wavelength.

Peak incandescent signal at a center detection wavelength.

LII intensity.

Average energy response from calibrated irradiance light source imaged onto each CCD pixel.

Modeled peak incandescent signal at a center detection wavelength.

Measured scattering signal before extinction correction.

Scattering intensity from a calibration gas, nitrogen in this study.

Scattering signal corrected for extinction through soot layer.

Scattering intensity.

Boltzmann constant.

Fractal aggregate prefactor dimension.

Projected aggregate prefactor parameter.

Nozzle separation distance.

Complex index of refraction of soot $m=n+i \kappa$.

Soot number density.

Number of primary soot particles per aggregate.

Number density of aggregate particles.

Number density of nitrogen.

Gas pressure.

Partial pressure of sublimed carbon.

Reference pressure used in Clausius-Clapeyron equation.

Scattering wave vector.

Normalized laser temporal profile where integration over all time is equal to unity. 


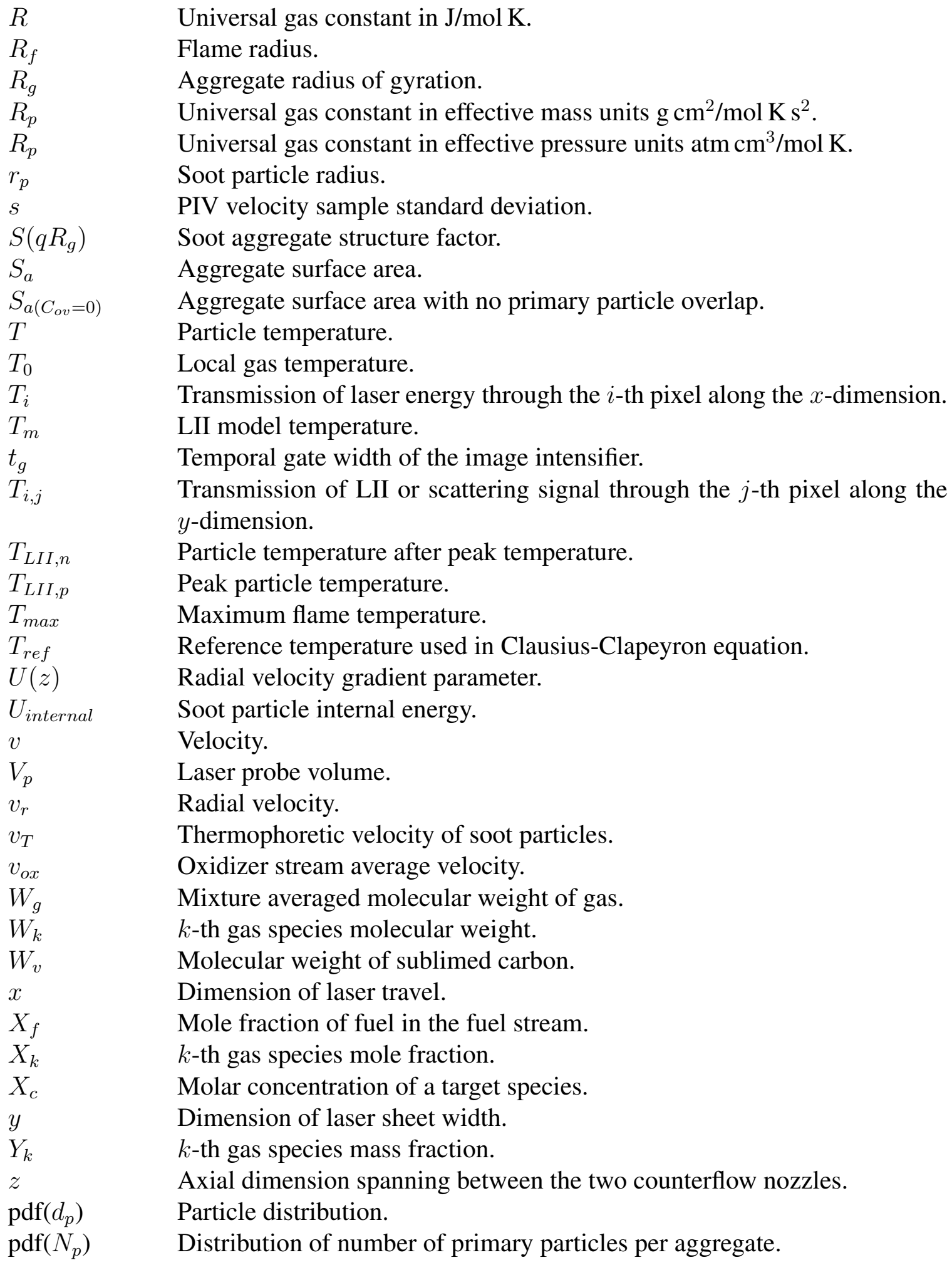




\section{Chapter 1}

\section{Introduction}

\subsection{Motivation}

The majority of domestic and international energy consumption results from the combustion of hydrocarbon fuels. Internationally, in 2010 total final energy consumption composed of $84 \%$ hydrocarbon fuels. Regardless of future climate and emission policy, international energy consumption is expected to continue to grow at an annual rate of $1.6 \%$ with hydrocarbon based energy continuing to satisfy a large portion of future demand [1]. It is well known that hydrocarbon combustion produces various pollutants [2]. Numerous studies have reported the adverse health, environmental, and climatic effects of pollutants including $\mathrm{CO}$, ozone, NOx and aerosol or soot particulate emissions from the combus-

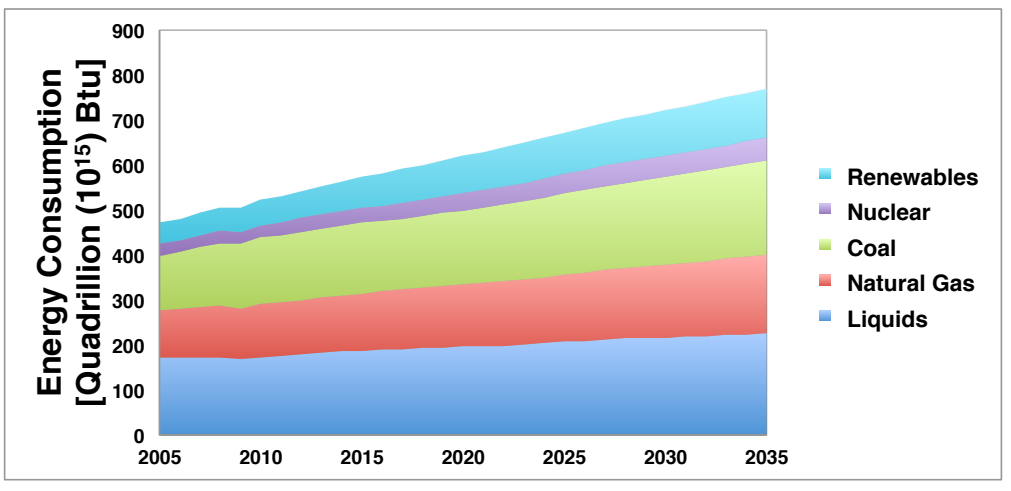

Figure 1.1: World total energy consumption. 
tion of hydrocarbons in boilers, furnaces, gas turbines and other internal combustion engines [3-5]. The free radical nature of soot is theorized to be a cause of cloud nucleation. Inhalation of free radicals into the human respiratory system is considered damaging and significantly increases the risk of lung cancer [6]. Studies also suggest a complex interplay between soot and other pollutants in some cases where soot is thought to play a role in reduction of the pollutant nitric oxide [7]. The US Clean Air Act of 1970 and later amendments and regulations by the EPA have published emission limits in the transportation and energy sectors on various pollutants including $\mathrm{CO}$, ozone, NOx, and particulate matter as low as 2.5 microns in diameter. Similar standards have been established through legislation by the European Commission and are implemented by many countries outside of the United States. The European standards include additional air quality objectives for 2.5 micron fine particle emissions [8]. From an ethical standpoint, effort dedicated to continuing research, technology advancements, and careful combustion optimization, should be conducted to further reduce or mitigate many known pollutants including fine particulate emissions. Additional advancements in technology for measuring and controlling fine particulate emissions and knowledge into the adverse health effects intensifies the potential for future pollutant regulations especially on soot particulate emissions below 2.5 microns. Concerted efforts are underway to better characterize fine soot particle emissions and understand the soot particle formation and oxidation mechanisms in combustion devices. Extensive experimental and modeling efforts have been undertaken by the scientific community and the transportation and energy sectors including the commercial aerospace industry over the last several decades [6,9-17]. A conceptual representation of the steps involved in soot formation are shown in Fig. 1.2 and described as follows [6, 17, 18]. Gas phase chemistry initiates polycyclic aromatic hydrocarbon (PAH) formation. Higher order planar PAH structures are formed by PAH chemistry and physical bonding mechanisms. The planar PAH structures combine to form three-dimensional stacked clusters which coalesce into nascent soot particles on the order of 3-5 $\mathrm{nm}$ with additional reaction time. Con- 


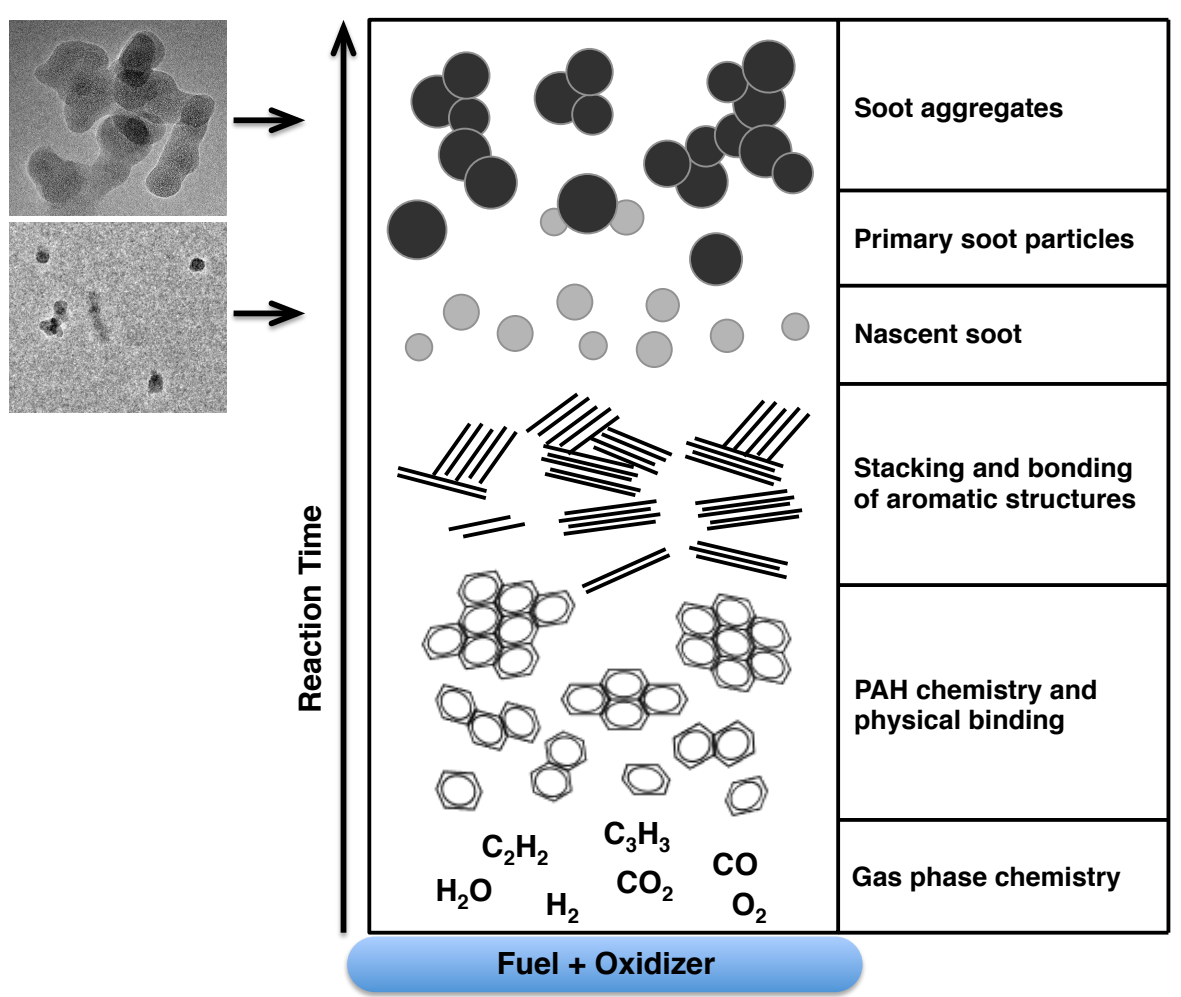

Figure 1.2: A conceptual representation of soot formation.

tinued particle surface growth leads to larger mature primary soot particles approximately 10-30 $\mathrm{nm}$ in size. With sufficient residence time, primary particles collide due to Brownian motion and coagulate to form aggregates.

Aside from the negative externalities associated with soot production and pollution from the burning of hydrocarbon fuels, there are numerous positive aspects to the synthesis of nanoscale carbon particles for several industrial applications. Recently, strategies were developed for using carbon nanoparticles in dye sensitized solar cells in an attempt to reduce cost and improve light absorption efficiencies [19]. The field of nanofluid research have proposed utilizing carbon nanoparticles for improved efficiency in heat exchange style solar collectors [20]. The increased efficiency is realized by introducing highly broadband light absorbing nanoparticles into the working fluid to increase solar light absorption. Other research has explored the application of carbon nanoparticles as electrodes for supercapacitors due to their large surface area to volume ratios for storing charge from a surrounding 
electrolyte [21]. Carbon nanoparticle research possibilities are exciting yet many challenges remain associated with measurement and production of nanoparticle products. New breakthroughs may one day make it possible to fine tune properties like flame generated particle size, structure, and optical properties to satisfy a range of industrial and technical applications.

\subsection{Selection of Equipment}

\subsubsection{Selection of Burner Configuration}

The chemical and physical processes of soot nucleation, growth, and oxidation are highly complex functions of macroscopic combustion properties like gas and particle flow residence time, temperature, fuel-oxidizer mixing, and pressure. While progress has been made towards understanding these processes, many of the details remain speculative or unknown $[6,7,22]$. Any design modifications to gas turbines or any other internal combustion engines to meet potential new regulations are expected to be led by computational fluid dynamics (CFD) modeling efforts. This will require soot kinetic models well validated by experimental data targeted at fundamental soot characteristics with well-defined uncertainties. While real world combustion flow fields are generally complex, and exhibit complicated turbulence and fuel-air mixing, laboratory burner configurations can employ

much simpler laminar flow field geometries. Canonical burner configurations significantly simplify the flow field characteristics by which flow field and chemical effects on soot nucleation, growth, and oxidation can be separately identified and accurately analyzed. Common laminar laboratory burner configurations include premixed burner stabilized flames, non-premixed co-flow diffusion flames, and counterflow flames [23-26].

All these burner configurations suffer from some degree of instability issues at increasing pressure due to turbulent transition. These instabilities are generally remedied by introducing a helium dilution to reduce gas density and delay transition according to 


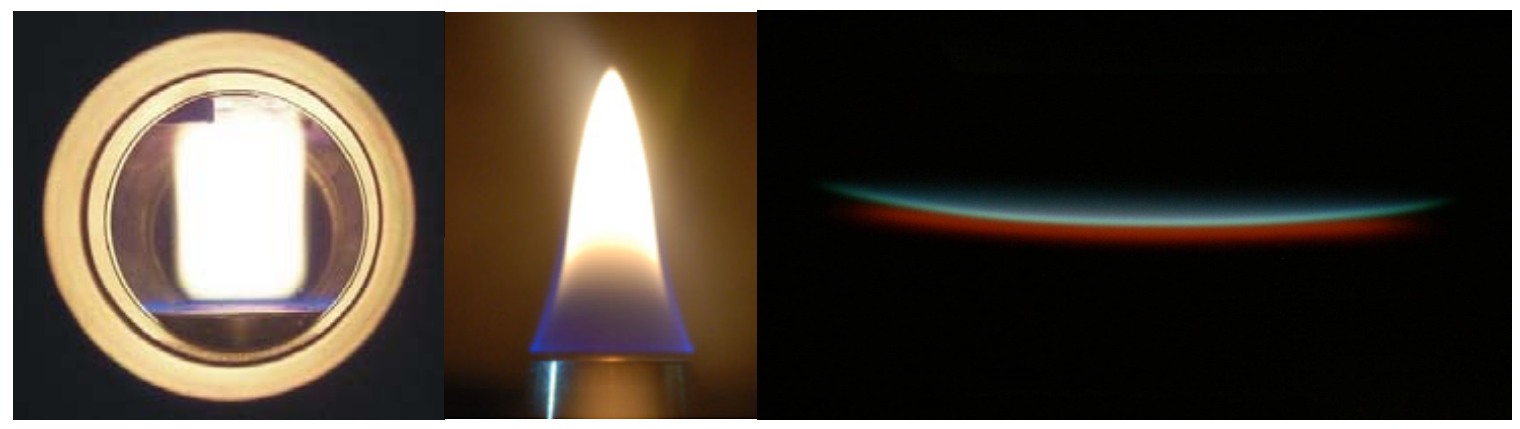

Figure 1.3: Left: Premixed burner stabilized flame originating from the burner surface near the bottom of the image [27]. Center: Non-premixed co-flow diffusion flame with the fuel nozzle shown in the middle of a surrounding air flow. [26]. Right: counterflow non-premixed flame with air flowing down from the top nozzle and fuel flowing up from the bottom nozzle.

Reynolds number. Both premixed burner stabilized flames and non-premixed co-flow diffusion flames are driven by buoyancy and suffer from buoyancy induced shear vortex instabilities especially at high pressure [28]. CFD models required to represent non-premixed co-flow diffusion flames are two-dimensional and require significant computational resources to accurately represent the flow field, leaving less resources available to include detailed chemical models. Models required to represent premixed burner stabilized flames are inherently one-dimensional and require modest computational resources to accurately resolve the reacting flow field. However, both configurations suffer from a lack of ability to control soot propensity. This stems from an inability to control the flow field residence time due to the buoyancy driven nature of the burners. Some measure of control usually resorts to the approach of perturbing the fuel-oxidizer ratio or fuel and oxidizer dilutions to change soot conditions. A lack of flexibility on flow residence time also results in a reduction in control of soot nucleation, growth, and oxidation. These flames generally exhibit the full range of soot particulates from early inception to agglomeration of particulates into very large aggregates. While a full range of soot formation steps is intuitive in its own right, a single step in the soot formation process cannot be individually targeted due to a lack of control of flow residence time. Additionally, the issue of agglomerated particles can add significant complexity to experimental measurement techniques. 
In contrast, counterflow flames are largely unaffected by buoyancy instabilities and the flow field can be accurately characterized through a one-dimensional similarity solution, leaving ample computational resources for analyzing detailed chemical models [28]. Flow instabilities from the counterflow fluid streams are generally found negligible in the practical sense. The configuration does suffer from a lack of spatial resolution in that the flame and soot layer formed is thin due to the chemical mixing layer and becomes thinner with increased flow strain (shorter flow residence time) and pressure. This drawback can be partially remedied by replacing nitrogen with helium as a diluent gas in the fuel and oxidizer streams. However, flow residence times and fuel-oxidizer compositions (diluted, non-premixed, partially premixed, or fully premixed) can be readily adjusted to control and examine a wide range of soot conditions. Most importantly and of great value to the research community, soot nucleation and early growth can be easily singled out and investigated. Due to its numerous advantages, use of the counterflow configuration will be the focus of this work.

\subsubsection{Selection of Measurement System}

With choice of the burner configuration also comes the careful selection of relevant measurement techniques for characterizing the physical-chemical processes of soot inception and growth. Various diagnostic tools have been developed to characterize combustion characteristics. Reviews of optical and intrusive techniques applicable to sooting flames are available in literature $[9,29]$. In terms of intrusive probing methods, Gas Chromatography/Mass Spectroscopy (GC/MS) is a technique used to collect gas species concentrations from a local probe region by first separating species via gas chromatography then measuring the species using mass spectroscopy. Scanning Mobility Particle Sizing (SMPS) is used to physically collect soot particles from a flame environment and measure particle sizes and distributions based on their aerodynamic size. However, both techniques suffer from spatial resolution and flow disturbance issues from physical probing under large spatial gradients 
of temperature and species concentrations like those found in counterflow flames.

In terms of optical diagnostics, Laser Induced Fluorescence (LIF), Tunable Diode Laser Absorption Spectroscopy (TDLAS), and Raman Scattering are commonly used to measure species concentrations and temperature. Rayleigh (Elastic) Light Scattering (ELS), Line of Sight Extinction or Attenuation (LOSA), Spectral Soot Emission (SSE), and Laser Induced Incandescence (LII) are common diagnostics for measuring soot properties. Rayleigh scattering is advantageous for acquiring a two dimensional elastic scattering irradiance signal from soot particles proportional to soot number density and particle size. Yet the technique suffers from extreme sensitivity to background scattered light and several complexities involving the structure of particles, insensitivity under some aggregate sizes ranges, and erroneous results due to particle polydispersity [30]. Several diagnostics used to measure soot properties take advantage of a unique property of soot compared to other combustion products. Soot exhibits a significant complex index of refraction over a large wavelength range and therefore readily absorbs electromagnetic radiation. Line of sight extinction is commonly utilized as a point measurement diagnostic by quantifying the total path integrated extinction of a laser beam due to soot and is utilized effectively in many studies [31-34]. However, the line of sight extinction technique is not well suited to the counterflow configuration especially at high pressure due to spatial resolution limits and large temperature and species concentration gradients causing beam steering. Spectral Soot Emission is a line of sight technique capable of measuring local flame temperature and soot volume fraction where soot is present by measuring the blackbody radiation emitted from soot at the local flame temperature. The results require an inversion technique to resolve a cross section. Laser Induced Incandescence is a nonintrusive measurement technique capable of measuring various soot properties. LII utilizes electromagnetic energy from a pulsed laser, some of which is absorbed by soot particles and converted to internal energy. The particles heat up; incandesce at elevated temperature governed by Planck's radiation law and cool through several heat transfer mechanisms. Soot volume fraction is determined from the absolute 
soot incandescence intensity while information on the soot particle size is inherent in the particle temperature decay. With a carefully designed LII system, the complex index of refraction of soot allows for measurement and analysis of soot properties filtered out from all other interfering signals including chemiluminescence, laser induced fluorescence, and elastic scattering. The issues of spatial resolution and flow disturbances from various measurement techniques using the counterflow configuration are acknowledged. As such, a high-resolution two-dimensional non-intrusive Laser Induced Incandescence system is the diagnostic measurement technique of choice for this investigation. The LII system developed in this study is capable of measuring particle temperature $(T)$, soot volume fraction $\left(f_{v}\right)$, primary particle size $\left(d_{p}\right)$, and number density $(N)$ across a thin non-premixed counterflow flame structure.

As previously mentioned flow field residence time plays a significant role in the processes of soot nucleation and growth. Velocity profiles and boundary conditions must be measured and specified accurately for comparison against modeled soot results. Improper boundary conditions have been shown to result in significant deviations between measured and modeled combustion characteristics [35]. For the characterization of flow residence time via velocity measurement, several diagnostics exist, most notably Laser Doppler Velocimetry (LDV) and Particle Image Velocimetry (PIV). Both diagnostics have been extensively implemented and tested in previous work [35]. Utilization of PIV was chosen as the desired diagnostic tool due to several factors including its high data collection rate, high spatial resolution potential, and ability to simultaneously collect two-dimensional flow field measurements. 


\subsection{Historical Background}

\subsubsection{A Brief History of Soot Research}

Soot investigations have been largely dominated by non-premixed co-flow diffusion flames $[11,26,32,34,36-40]$ and premixed burner stabilized flames [12, 24, 41-43], most likely

due in part to relaxed spatial resolution requirements for point measurement diagnostics. Utilizing the non-premixed co-flow configuration, a sampling of soot research conducted at atmospheric pressure is as follows. Kang et al. [32] examined soot volume fraction, number density, and primary particle size with point measurements of line of sight extinction and elastic scattering while also quantifying polycyclic aromatic hydrocarbon (PAH) fluorescence and soot limits and reported the influence of flame structure on soot properties. In one of the first practical utilizations of laser induced incandescence, Vander Wal et al. [36] examined the potential of LII signal decay point measurements to determine primary soot particle size and reported successful results. More recently, Mikofski et al. [37] examined and reported two-dimensional planar profiles of the structure of $\mathrm{OH}$ and $\mathrm{PAH}$ fluorescence signals and laser induced incandescence signals of soot from inverse diffusion flames. The group reported spatial and temperature ranges of soot inception and growth and the relationship with PAH species. At elevated pressures McCrain and Roberts [38] measured and compared integrated soot with line of sight extinction and soot volume fraction with LII at pressures up to $2.5 \mathrm{MPa}$ and reported variations between pressure scaling of the two measured parameters. Kim et al. [39] measured species and soot concentrations, soot temperature, and structure to determine soot growth and oxidation rates for pressure up to $8 \mathrm{~atm}$. Thomson et al. [34] examined and compared soot morphology including volume fraction and particle size using LII and line of sight extinction point measurements at pressures as high as $4 \mathrm{MPa}$. Several researchers have utilized spectral soot emission at ambient flame temperature to determine soot temperatures and soot volume fractions at pressures up to $35 \mathrm{~atm}[11,26,40]$. Joo [44] extended the pressure range applied to co- 
flow diffusion flames in a $\mathrm{PhD}$ dissertation and examined soot volume fraction and flame temperature for methane-oxygen flames up to $100 \mathrm{~atm}$ using spectral soot emission. This remains one of the highest known pressures investigated. While some of these studies have proved pioneering to the research community, the issue of the two-dimensional nature of the flow field ads unnecessary computational burden and none of these studies adequately addressed measurement complexities due to aggregate formation.

Utilizing the premixed burner stabilized flame configuration, atmospheric pressure soot studies have been conducted by several research groups. Xu et al. [41] measured species concentrations, soot volume fraction, and soot primary particle size with an array of diagnostic methods to determine soot nucleation and surface growth rates with good correlation found to commonly proposed growth mechanisms. Inal and Senkan [42] focused on measurements of aromatic and PAH species concentrations using gas chromatography-mass spectrometry (GCMS) while also measuring soot volume fraction and primary particle size with line of sight extinction and scattering. However, a key particle size assumption made in this study self admittedly introduced large uncertainties in the regime of aggregate particles. Also in a novel study, Ergut et al. [43] investigated the effect of temperature on soot and PAH concentrations using GC and GCMS analysis and reported that hotter flames exhibited reduced soot loading due to increased oxidation beyond the rate of soot formation. At elevated pressure, Tsurikov et al. [12] reported soot volume fraction and temperature measurements for flames up to 5 bar using LII and Coherent anti-Stokes Raman Spectroscopy (CARS). Hoffman et al. [24] investigated the use of LII at pressures up to 10 bar and reported data for measured soot volume fractions and primary particle sizes with attention given to aggregate soot particles. Good agreement was reported with thermophoretically sampled soot analyzed using Transmission Electron Microcopy (TEM). While the premixed burner stabilized configuration offers computational simplicity over non-premixed co-flow flames due to their one-dimensional nature, as previously mentioned, the issues of a lack of control on flow residence time, aggregated particles, and buoyancy instabilities 
remain.

Less effort has focused on the use of the counterflow configuration for soot research. A comprehensive review of research at atmospheric pressure resulted in only a handful of studies known to this author. Mcnesby et al. [45] investigated the influence of fuel composition on soot formation and $\mathrm{OH}$ concentration using elastic scattering and $\mathrm{OH}$ fluorescence in two-dimensions. Leusden and Peters [33] used quartz probe sampling and GC analysis to measure various species at low strain rate and atmospheric pressure to achieve acceptable resolution for probing while minimizing the effects of flow disturbance. Yamamoto et al. [46] studied the effect of strain rate on PAH formation using GCMS. Lin and Faeth [47] reported hydrodyamic sooting limits for a range of fuels and mixture fractions. Of great importance, the counterflow burner configuration was utilized to target soot nucleation and growth. Several researchers primarily used one-dimensional point or line of sight diagnostics of combined extinction and scattering or laser induced incandescence at atmospheric pressure where the flame thickness is conducive to point measurements. Soot limits, soot volume fraction, and particle sizes were measured to determine soot growth rates for comparison to soot growth mechanisms [25,32,48]. Visible fluorescence was also used to observe relative PAH concentrations. At elevated pressure, counterflow studies related to soot formation are even less prevalent. To date, only two studies were found in literature. Du et al. [10] investigated sooting limits at pressures up to 2.5 atm utilizing elastic scattering while Sung et al. [49] reported visual sooting limits with Raman scattering species concentration measurements at pressures up to $5 \mathrm{~atm}$. The highest reported pressures investigated using the counterflow configuration, while not specifically related to soot research, were $15 \mathrm{~atm}$ [50] and $30 \mathrm{~atm}$ [28]. Results applicable to soot studies were reported by Figura and Gomez [28] who achieved favorable spatial resolution and flame stability by diluting flames with helium at high pressure. Lack of research at elevated pressure utilizing the counterflow burner configuration, its computational one-dimensionality, and its ability to target early soot nucleation and growth while avoiding complexities due to particle ag- 
gregation is evident. A detailed and complete dataset on the effects of pressure and flow residence time on soot characteristics is also lacking in literature and is a clear motivation for this work.

\subsubsection{A Brief History of Laser Induced Incandescence}

Equally important to this work is the research leading up to the laser induced incandescence system utilized in this study. In 1974 Weeks and Duley [51] developed the first basic nanoscale particle heat balance and showed size information could be determined from the incandescent signal decay of aerosols. In 1977 Eckbreth [52] observed interference in Raman scattering measurements due to soot incandescence and developed an incandescence model to improve the signal to noise ratio of Raman measurements. In 1984 work by Melton [53] first exhibited the potential of LII as a measurement technique and reported proportionality between the soot incandescent signal and soot volume fraction. While providing the first relation between incandescent signal and volume fraction, the analysis only applied to the high laser fluence, high soot vaporization regime where considerable uncertainties exist on the vaporization heat transfer term and enthalpy of formation of sublimed carbon species. The formulation also requires an assumption or measurement of particle size due to a nonlinear dependence on volume fraction. Bad assumptions or inaccurate measurements of particle size would result in large errors on soot volume fraction. Laser induced incandescence was largely overlooked as a practical measurement technique until the 1990s when in 1991 Dec et al. [54] reported the first qualitative measurements of in-cylinder soot concentrations from a diesel engine.

The use of laser induced incandescence for soot particle sizing began in 1995 where Roth and Filippov [55] used the ratio of LII signals at two or more times after initial particle heating to deduce primary particle size with a nanoscale heat transfer model. Also in 1995, Will et al. [56] was the first to apply LII to two-dimensional particle sizing through the ratio of the incandescent signal at two decay times. In 2004, Dankers and Leipertz [57] 
attemped to determine primary particle size and distribution width with the assumption of a distribution type and two signal decay rates. The application of LII to measuring size distributions, however, comes into question when analyzing the uncertainties in measuring particle size alone and the ability to accurately resolve small changes in the temperature decay rate used to determine the size distribution.

Quantitative advancements in measuring soot volume fraction began in 1994 with Vander Wal and Weiland [31] who first experimentally demonstrated a linear proportionality between the LII signal and soot volume fraction in comparison to light extinction measurements. Santoro et al. [58] utilized the linear proportionality by calibrating LII measured soot volume fractions by single point light extinction while also measuring soot particle size via elastic scattering. These works represented the next major advancement in calibration and determination of soot volume fraction by laser induced incandescence. While this technique relies on a formulation more fundamentally understood than the original relation by Melton [53], this procedure does suffer from its own inherent uncertainties. Most notably, the uncertainties in the optical properties of soot and changes in particle size that introduce time gate integrated signal uncertainties. The latter suggests short integration time gates are preferable for transfer calibration to LII at the expense of signal. The calibration method also suffers from spatial and temporal resolution issues inherent to line of sight extinction. In 1995, Santoro et al. [59] was the first to acquire two-dimensional images of soot volume fraction. In 2003 Hoffman et al. [60] first applied LII to measuring soot volume fraction in high pressure laboratory flames. In 2005 Snelling et al. [61] introduced a novel approach to LII calibration that relies strictly on the physics of LII and two-color pyrometry. By relating the spectral radiance of the soot particle incandescent signal at known temperature to an absolute irradiance or radiance calibration of the recording device, the soot volume fraction can be directly determined. While the original study was applied to point measurements of laser induced incandescence, the concept is robust and can be applied to two-dimensional imaging. The method only suffers from uncertainty 
in the known optical properties of soot, but offers potential for high temporal and spatial resolution. The absolute irradiance calibrated LII method is adopted in this work and is further extended and modified for two-dimensional imaging.

\subsection{Dissertation Objectives}

The effects of turbulence, complex fuel air mixing, and other real world combustion operating parameters all merit investigation. However, attention must first be devoted to the simplest of underlying effects due to the complexity and challenges in understanding the inception and growth of soot. One of the most elementary and important effects of soot formation and growth relevant to modern combustion engines is that of pressure. Utilizing the simple laminar, steady counterflow configuration, the goal of this work is to investigate and analyze the effect of elevated pressure on the soot characteristics of hydrocarbon combustion over a wide range of flow residence times.

\subsection{Dissertation Outline}

The organization of this dissertation continues as follows:

\section{Chapter 2 - Research Design and Methodology}

The general experimental procedure is presented for laser induced incandescence and particle image velocimetry measurements. The laser induced incandescence model and elastic light scattering and light extinction correction submodels are developed for extracting measured soot particle temperature, volume fraction, primary particle size, and number density. A thermophoretic sampling device is also developed.

\section{Chapter 3 - Uncertainty Analysis}

A detailed uncertainty analysis of laser induced incandescence and particle image 
velocimetry measurements is presented. Improvements to uncertainty estimates are suggested.

\section{Chapter 4 - Experimental Results}

Thermophoretically sampled soot is analyzed by transmission electron microscopy and soot characteristics including aggregate dimensions, primary particle diameter and aggregate size distributions, and particle morphology are presented. A detailed analysis of underlying laser induced incandescence measured soot characteristics and formation and growth mechanisms is presented. The effects of pressure and strain rate on formation and growth mechanisms is reported.

\section{Chapter 5 - Summary and Recommendations}

The significant conclusions of this dissertation and practical applications for industry are summarized. Recommended improvements to the experimental apparatus and avenues for future research are suggested. 


\section{Chapter 2}

\section{Research Design and Methodology}

\subsection{General Experimental Methodology}

A nonpremixed counterflow burner designed to operate at atmospheric and elevated pressures was used to conduct all experiments [62]. The high pressure chamber and LII system are shown in Fig. 2.1. A set of two nested convergent co-annular nozzles made up the counterflow burner. The counterflow burner assembly is shown in Fig. 2.2. The inner nozzles were used for the fuel and oxidizer streams while inert flows of nitrogen (or helium for high pressure experiments) were introduced through the outer annuli formed between the inner and outer nozzles to prevent secondary flames in the enclosed chamber. The convergent co-annular nozzles were machined from aluminum with a contour designed to minimize the boundary layer and boundary layer instabilities (Taylor-Görtler vortices) at the nozzle exit plane due to the nozzle radius of curvature $[63,64]$. The inner nozzle exit diameter was selected to be $6.5 \mathrm{~mm}$ with a convergent area ratio between the inner nozzle tube diameter and nozzle exit diameter of 19.1. The design ensured a consistent top hat velocity profile upon exit from the inner nozzles. The two sets of co-annular counterflow nozzles were mounted vertically in the chamber with the capability for varying the counterflow burner separation distance $L$ using spacers. Four two inch diameter ports sealed by one inch thick, 


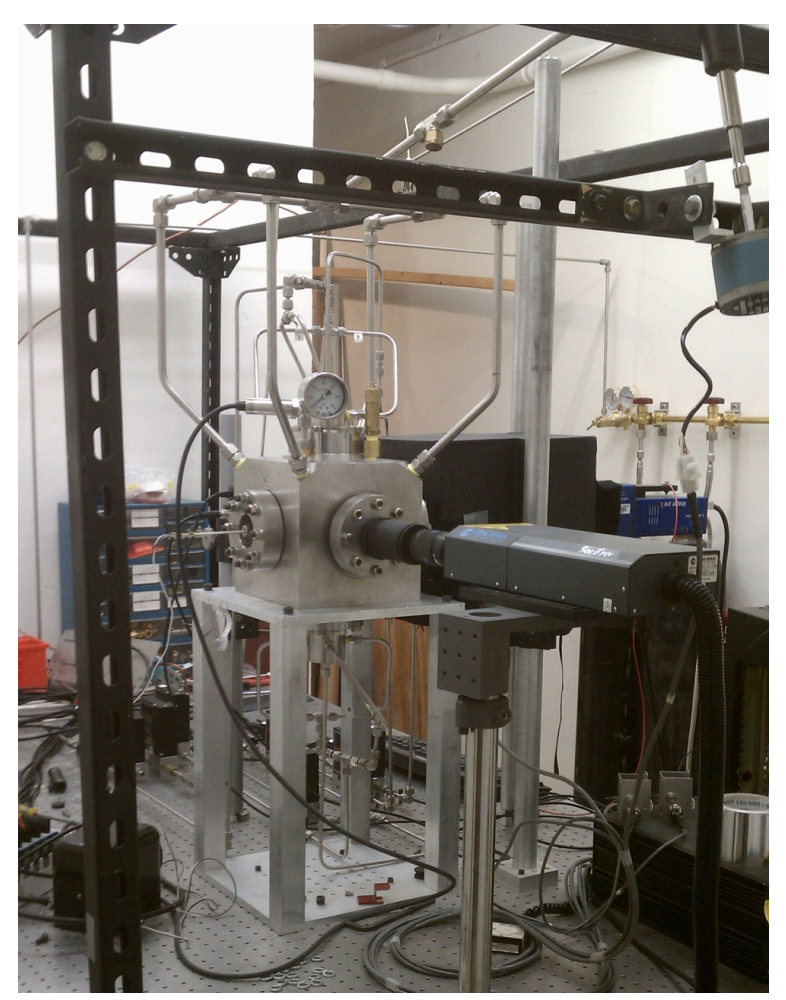

Figure 2.1: High pressure counterflow burner with assembled LII system. The motorized back pressure regulator is shown in the top right and flow meters are visible in the background.

three inch diameter fused silica windows arranged with one centered on each side of the square chamber allowed access for LII and PIV diagnostics and viewing of the flame. The fused silica windows were hydro tested to a pressure of $150 \mathrm{~atm}$ while the chamber itself was hydro tested to a pressure of $200 \mathrm{~atm}$ using aluminum blanks. In experiments, the oxidizer, fuel and inert gases were metered and controlled by a series of Sierra model 100 mass flow controllers (with a factory calibrated accuracy of $\pm 1 \%$ of full scale and repeatability of $\pm 0.2 \%$ of full scale including linearity at operating conditions) interfaced in a LabView data acquisition program. The chamber operating pressure was regulated via a Stravalve back-pressure regulator controlled by a stepper motor through LabView. For the case of atmospheric pressure data, the backpressure regulator was removed. Overpressure safety precautions were taken by additionally equipping the chamber with a pressure relief valve and burst disk staggered approximately 20 and 50 psi above operating pressure. Bulletproof 


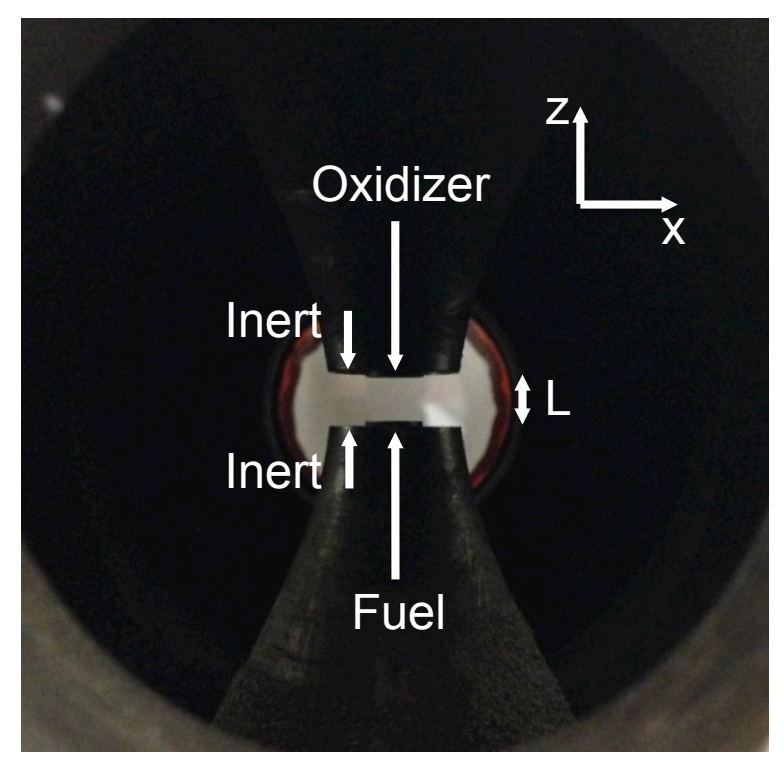

Figure 2.2: Counterflow burner nozzle assembly. Oxidizer is metered from the top inner nozzle with fuel metered from the bottom inner nozzle. Inert gas is introduced through the outer annulus formed between the inner and outer nozzles. The nozzle separation $L$ is shown in the $z$-dimension.

polycarbonate and aluminum plating shielded the operator for safety.

LII measurements utilized a New Wave Research Solo III 50 mJ, $532 \mathrm{~nm}$, pulsed (duration $8 \mathrm{~ns}$ FWHM) dual head Nd:YAG laser with collimated and apertured Gaussian sheet optics (adjustable height, $\sim 250 \mu \mathrm{m}$ width FWHM). Only one laser head was used for LII measurements. Laser fluence was controlled by adjusting the energy output of the flash lamp. A peak laser fluence around $0.1 \mathrm{~J} / \mathrm{cm}^{2}$ was used for all experiments to maintain peak soot particle temperatures below the $\sim 4000 \mathrm{~K}$ sublimation threshold of soot while maximizing incandescent signal in the low fluence regime and minimizing the potential for C2 Swan band laser induced fluorescent interference [65]. The soot particle incandescent signal was collected through a Nikon micro-Nikkor $105 \mathrm{~mm}$ lens, interchangeable $450 \mathrm{~nm}$ and $650 \mathrm{~nm}$ bandpass filters (10 nm FWHM), LaVision Intensified Relay Optics (IRO), and a LaVision Imager ProX4M 2048x2048 pixel CCD camera. The two bandpass filters were used to measure particle temperature via two color pyrometry where the center wavelengths and widths of the filters were chosen to further avoid potential C2 Swan band fluorescent transitions [65]. The bandpass filters were interchanged with a motorized 


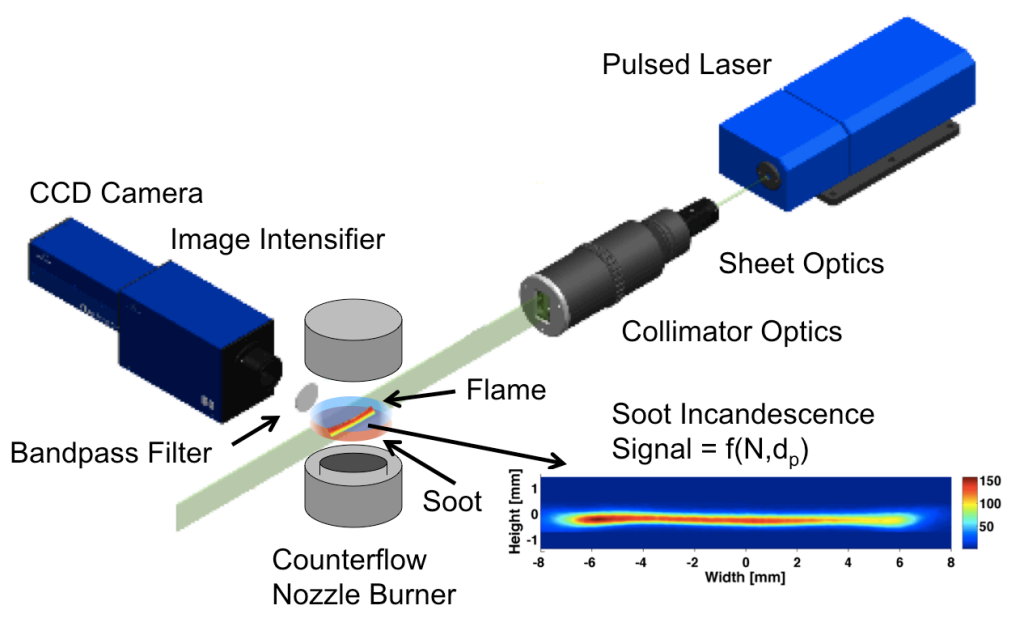

Figure 2.3: Schematic and general layout of absolute irradiance calibrated LII system. The same configuration applies to PIV without the image intensifier. The insert shows the typical image of 500 LII signals.

filter wheel built into the optical assembly. The equipment was controlled via Davis 7.2 and LaVision SootMaster software through a programmable timing unit with $10 \mathrm{~ns}$ resolution. The PIV system utilized the same laser and CCD camera with a Nikon micro-Nikkor $200 \mathrm{~mm}$ lens and $532 \mathrm{~nm}$ bandpass filter (10 nm FWHM). LaVision FlowMaster software controlled PIV data collection and processing.

The general experimental procedure adopted is as follows. The camera and laser sheet are aligned perpendicular, focused, and centered on the air nozzle exit axis of symmetry as shown in Fig. 2.3. The air nozzle exit plane is identified by $z=+L / 2$ while the fuel nozzle exit plane is identified by $z=-L / 2$. The $z$-dimension identifies the axis of symmetry coordinate with $L$ the nozzle separation distance and $z=0$ the midpoint between the two burner nozzles. The camera images are spatially calibrated in reference to the known inner nozzle exit diameter $(D)$. Opposed non-premixed fuel, oxidizer, and inert gas dilution streams are introduced via the inner nozzles, with momenta balanced $\left(\rho_{o x} v_{o x}^{2}=\rho_{\text {fuel }} v_{\text {fuel }}^{2}\right.$ where $\rho_{o x}$ is the oxidizer stream density, $v_{o x}$ the average oxidizer nozzle exit velocity, $\rho_{\text {fuel }}$ the fuel stream density, and $v_{f u e l}$ the average fuel nozzle exit velocity). The average nozzle exit velocities are determined by the ratio of the volume flow rate to nozzle cross sectional area. A co-flow of nitrogen or helium is introduced 
through the outer annulus of each nozzle with the same momentum balance. For a selected global strain rate (a function of inverse flow residence time), defined by $a_{g}=4 v_{o x} / L$, a LabView interface automatically assigns all flow meter settings. Once the chamber pressure is stabilized near the desired pressure, the mixture is ignited with a spark, and a stable planar axisymmetric flame is produced between the counterflow nozzles. For high pressure experiments beyond 8.45 atm the burner is ignited at a lower pressure around $6 \mathrm{~atm}$ and ramped up to the desired operating pressure. A global strain rate is chosen within the sooting regime of the given fuel-oxidizer mixture and two color LII image samples are collected from heated soot particles excited by the laser and filtered through the $450 \mathrm{~nm}$ and $650 \mathrm{~nm}$ bandpass filters with an exposure time of $10 \mathrm{~ns}$ dictated by the intensifier gate. The IRO delay time is varied to collect gated signals temporally as the particle temperature decays in order to characterize soot particle size. The prompt sample corresponding to peak temperature at the peak of the laser pulse is used to characterize soot volume fraction. In order to achieve sufficient signal, samples of five hundred LII images are collected and averaged. The validity of time averaging under the assumption of steady laminar flow has been previously verified with PIV measurements [35]. For characterizing flow residence time in the sooting regime with PIV, five hundred images are collected and processed using Davis 7.2 and the FlowMaster software to obtain mean and RMS 2D velocity data.

\subsection{Supporting Numerical Results}

The LII analysis requires knowledge of local gas temperature and physical properties including local species concentrations, the local gas thermal conductivity, and local gas heat capacity ratio. Since the near sooting flames considered in this study are far from extinction limits, only the mixing phenomena and thermo-chemistry control the flame structure. The mixing layer thickness can be well characterized by knowing the nozzle exit velocities and the gradients, easily measured by PIV as described in $[35,66]$. By implementing the 
above experimentally measured flow boundary conditions, together with the most recent compilation of thermochemical, transport, and untuned finite-rate chemical kinetic parameters $[67,68]$ in quasi one-dimensional simulations [69], the required gas phase properties

of ethylene-air flames were evaluated. The key flame properties extracted for LII analysis are the local temperature and gas composition. Both are used to calculate heat transfer variables for soot particle temperature decay analysis. Certain known soot precursors calculated from the present numerical approach are shown with LII data to lend support for scientific discussion but their uncertainties can be quite significant.

\subsection{Laser Induced Incandescence Methodology}

\subsubsection{Absolute Irradiance Calibration of ICCD Camera}

Following the calibration approach proposed by Snelling et al. [61], a light source of known spectral irradiance (model RS-10D from Gamma Scientific) was used for calibration of the detection system. The light source is shown in Fig. 2.4. A sample of five hundred images of the irradiance incident on the intensified CCD chip from the calibrated light source were collected over $10 \mathrm{~ns}$ and $20 \mathrm{~ns}$ gate widths and through two $10 \mathrm{~nm}$ bandwidth bandpass filters at $450 \mathrm{~nm}$ and $650 \mathrm{~nm}$. Samples were also collected over three different separations between the calibrated light source and the detector to test for repeatability. Irradiance incident on the detector scales with the inverse-square law of distance and the calibrated physical area represented by one pixel on the CCD. The energy collected by each pixel over a specified gate width is represented by a unit of "counts". These signal counts are related back to an irradiance scale by a calibration factor $\eta_{i}$ at the $i$-th bandpass filter center wavelength $(450 \mathrm{~nm}$ or $650 \mathrm{~nm}$ in this study),

$$
\eta_{i}=\frac{A_{c} I_{c}\left(\lambda_{i}\right) t_{g}}{I_{L S}}
$$




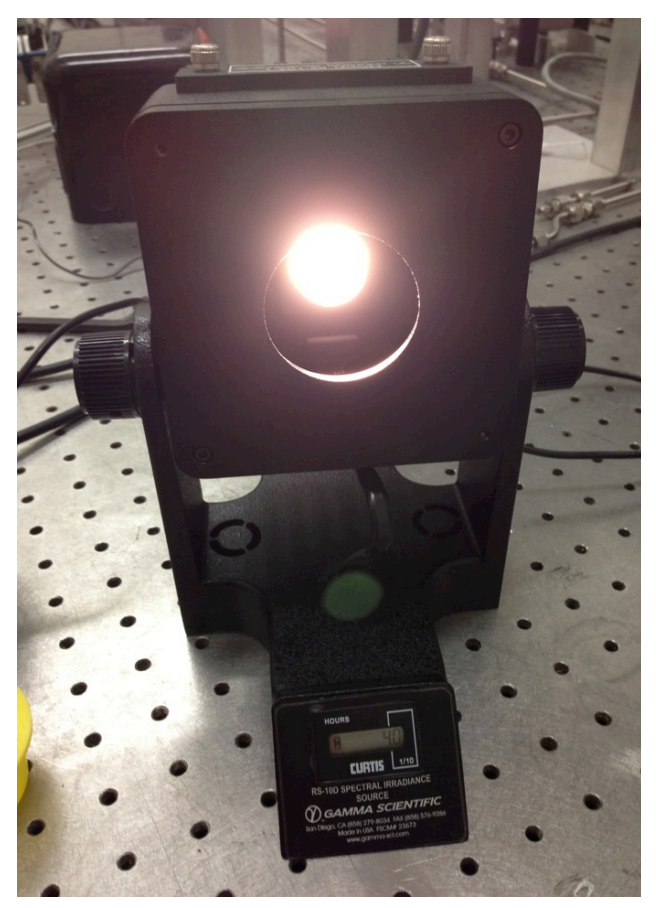

Figure 2.4: Gamma Scientific RS-10D calibrated light source used for imaging irradiance onto intensified camera.

where $I_{L S}$ is the five hundred image average energy response of the CCD represented as counts from the calibrated irradiance light source imaged onto the $\mathrm{CCD}, A_{c}$ is the calibrated area of one pixel of the CCD camera to image dimensions in the configuration used to conduct calibrations, $t_{g}$ the temporal gate width of the image intensifier, and $I_{c}\left(\lambda_{i}\right)$ the known absolute spectral irradiance at the bandpass filter center wavelength $\lambda_{i}$ from the calibrated light source. A test was conducted to determine mathematically that calibrating to the spectral irradiance of the filter center wavelength is simpler and results in negligible difference from results if the integrated signal across the filter bandwidth were used to calibrate. The procedure results in a calibration for direct measurement of soot volume fraction according to the absolute incandescent emission of soot particle radiation from Plank's law if the temperature of the laser heated soot particles is known. 


\subsubsection{Beam Profile and Fluence Calibration}

An accurate LII model also requires careful spatial characterization of the excitation laser source, as well as the calibration of energy per unit area (fluence). The measurements were conducted with a Thorlabs BC106-VIS CCD beam profiler and a total laser pulse energy transfer calibration from a Scientech AC2501 Astral Calorimeter with 3\% documented uncertainty. Measurements from five hundred successive laser pulses were recorded for a range of laser power settings covering the parameters defined by low to high fluence LII. Figure 2.5 shows the a) 2D profile and b) contour plot of the fluence calibrated beam sheet used in this study. The beam sheet appears mostly Gaussian with some minor Fresnel diffraction noticeable at the two ends in height. Collimation of the sheet minimized the diffraction pattern. The beam sheet profile along the center in height is clearly consistent and was configured so the soot layer was uniformly heated from this constant profile center in height (approximately $2.5 \mathrm{~mm}$ ). Shot to shot variation is significant and on the order of $10 \%$. Fluence variations between five hundred shot sample averages however are negligible. Additional details are given in Chapter 3.1.1.

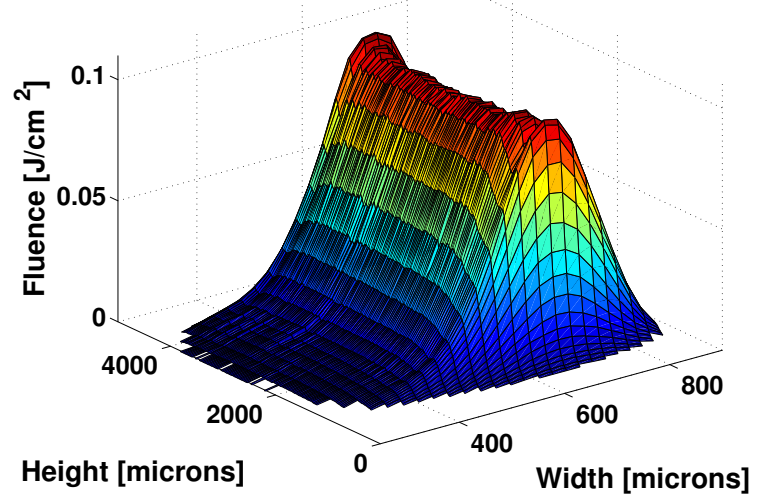

(a) 2-D profile

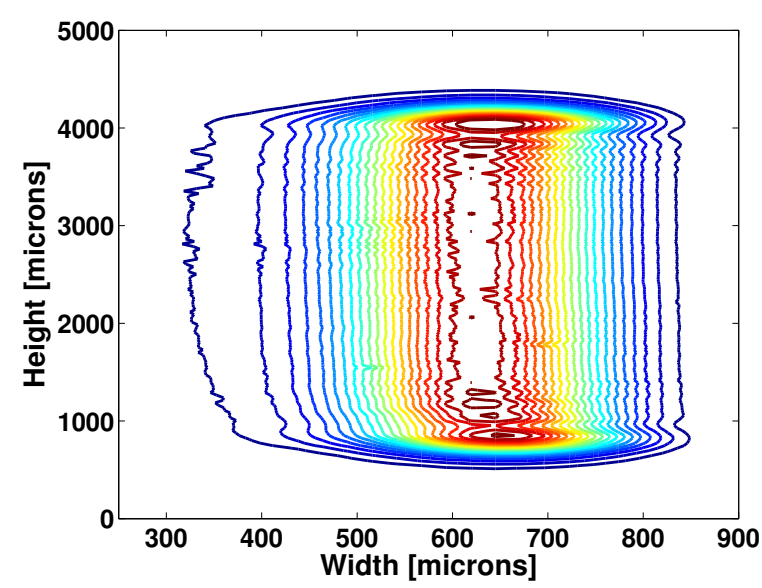

(b) Contour plot

Figure 2.5: Profile of laser sheet used in LII studies.

Images of the average laser sheet fluence profile were recorded by the beam profiler for a range of laser power settings. The images were imported and analyzed in MATLAB 
where a lookup table was generated of laser fluences and corresponding spatial locations across the excitation laser beam sheet width at center height. Figure 2.6 shows all laser beam sheet fluence profiles that were measured and calibrated for potential use in LII experiments. The fluences range from low to high fluence LII $\left(\sim 0.08\right.$ to $\left.0.26 \mathrm{~J} / \mathrm{cm}^{2}\right)$, the latter case defined by the point at which significant sublimation plays a role in the physics of LII. The highest and lowest calibrated fluence profiles were fitted with a Gaussian as a reference to the ideal laser profile expected. The lowest fluence profiles exhibited the best Gaussian fits.

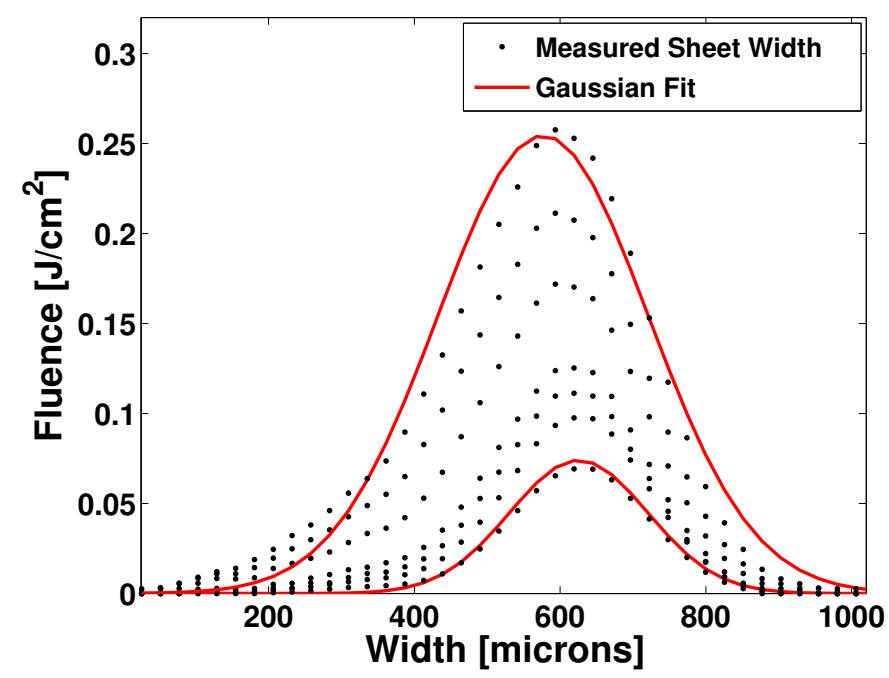

Figure 2.6: Fluence calibrated beam sheet width profiles for laser settings from low to high fluence LII (black dots) with Gaussian profile fits to the lowest and highest fluence profiles (red lines).

\subsubsection{Particle Temperature}

\section{Experimental Temperature}

The result of the above radiometric calibration can be used to determine properties of interest for each pixel in two dimensions including soot particle temperature, mean particle size, and volume fraction with the aid of a comprehensive soot particle heat transfer model. The experimental soot particle temperature is extracted from a combination of two-color 
pyrometry and incandescent signal decay. The peak particle temperature corresponding to maximum incandescence signal $\left(T_{L I I, p}\right)$ is determined by the following two-color pyrometry equation based on Planck's radiation law and the Wien approximation [61],

$$
T_{L I I, p}=\frac{h c}{k_{B}}\left(\frac{1}{\lambda_{2}}-\frac{1}{\lambda_{1}}\right) / \ln \left[\frac{I_{L I I, p}\left(\lambda_{1}\right) E(m) \eta_{1} \lambda_{1}^{6}}{I_{L I I, p}\left(\lambda_{2}\right) E(m) \eta_{2} \lambda_{2}^{6}}\right]
$$

Here, $h$ is the Planck constant, $c$ the speed of light, $k_{B}$ the Boltzmann constant, $I_{L I I, p}\left(\lambda_{i}\right)$ the incandescent signal at a given center detection wavelength in counts, the absorption function of soot $E(m)=-\operatorname{Im}\left[\frac{m^{2}-1}{m^{2}+2}\right]$ (a function of the complex index of refraction of soot $m=n+i \kappa)$ at a center detection wavelength, and $\lambda_{1}$ and $\lambda_{2}$ the center detection wavelengths $650 \mathrm{~nm}$ and $450 \mathrm{~nm}$ respectively. While the complex index of refraction is a function of wavelength, both $650 \mathrm{~nm}$ and $450 \mathrm{~nm}$ wavelengths are found to fall near a trough in the curve for the refraction index of soot based on dispersion theory [72]. The absorption function is therefore assumed constant between the two detection wavelengths. The equation assumes the Rayleigh limit of small particles $\pi d_{p} / \lambda<0.3$ is valid for volumetric light absorption and emission where $d_{p}$ is the particle diameter. After the peak particle temperature is reached, the temporal decay of soot particle temperature results in a rapid decay in incandescent signal according to Planck's radiation law. The incandescent signal decay and decreasing signal to noise ratio is most evident through the $450 \mathrm{~nm}$ bandpass filter and introduces a large uncertainty to measured particle temperatures by two color pyrometry using Eq. 2.2. To address this issue, a different approach was considered here in contrast to the original work by Snelling et al. [61], in which Eq. 2.2 was exclusively utilized. The resulting temporal decay of particle temperature $\left(T_{L I I, n}\right)$ after the peak particle temperature is determined here by the following equation based on the higher signal to noise ratio incandescent signal decay through the $650 \mathrm{~nm}$ filter from Planck's law,

$$
T_{L I I, n}=\frac{h c}{\lambda_{1} k_{B} \ln \left[\frac{I_{L I I, p}}{I_{L I I, n}} \exp ^{\left(h c / \lambda_{1} k_{B} T_{L I I, p}\right)}-\frac{I_{L I I, p}}{I_{L I I, n}}+1\right]} .
$$


The temperature decay calculated from Eq. 2.3 was tested against the use of Eq. 2.2 used exclusively and was found to provide less uncertain temperature measurements due to the strong incandescent signal decay through the $450 \mathrm{~nm}$ wavelength filter with decreasing temperature.

\section{Modeled Temperature}

With a LII heat transfer model solution, the modeled soot incandescent signal is given by,

$$
I_{m}\left(\lambda_{i}\right)=\frac{24 \pi^{2} E(m)}{\lambda_{i}} \int_{t_{0}}^{t_{f}} \int_{-\infty}^{\infty} \int_{0}^{\infty} \int_{1}^{\infty} B_{T} \operatorname{pdf}\left(N_{p}\right) \operatorname{pdf}\left(d_{p}\right) d N_{p} d d_{p} d y d t
$$

Here, the spectral radiance $B_{T}$ from Planck's law emanating from a particle at a given temperature is integrated over the intensifier gate time $t$, the beam sheet width $y$, an assumed primary particle distribution $\operatorname{pdf}\left(d_{p}\right)$, and an assumed distribution of the number of primary particles in a soot aggregate $\operatorname{pdf}\left(N_{p}\right)$ to match the experimental soot incandescent signal collected from the soot layer of a flame. The expression for spectral radiance is given by,

$$
B_{T}\left(N_{p}, d_{p}, y, t\right)=\frac{2 h c^{2}}{\lambda_{i}^{5}}\left[\exp \left(\frac{h c}{\lambda_{i} k_{B} T_{m}\left(N_{p}, d_{p}, y, t\right)}\right)-1\right]^{-1}
$$

The modeled particle temperature $\left(T_{m}\right)$ is calculated by two-color pyrometry using Eq. 2.6 in an attempt to exactly match the experimental temperature measurement procedure.

$$
T_{m}=\frac{h c}{k_{B}}\left(\frac{1}{\lambda_{2}}-\frac{1}{\lambda_{1}}\right) /\left[\ln \left(\frac{I_{m}\left(\lambda_{1}\right) \lambda_{1}^{6}}{I_{m}\left(\lambda_{2}\right) \lambda_{2}^{6}}\right)\right]
$$

\subsubsection{Primary Particle Size}

In order to determine primary particle size, the experimental particle temperature decay curve determined by Eqns. 2.2 and 2.3 must be fitted to the results of an LII heat transfer model representing the same particle temperature decay from Eq. 2.6. Primary particle size is solved by iterative weighted least-squares minimization of the modeled and experimental 
particle temperature decays where the peak temperatures are preferentially matched with a weight factor of 6. Different weight factors were tested and a value around 6 was found to give good volume fraction convergence between the $650 \mathrm{~nm}$ and $450 \mathrm{~nm}$ filters while also providing a good fit for particle size. An example of the fitting approach is given by Fig. 2.7 .

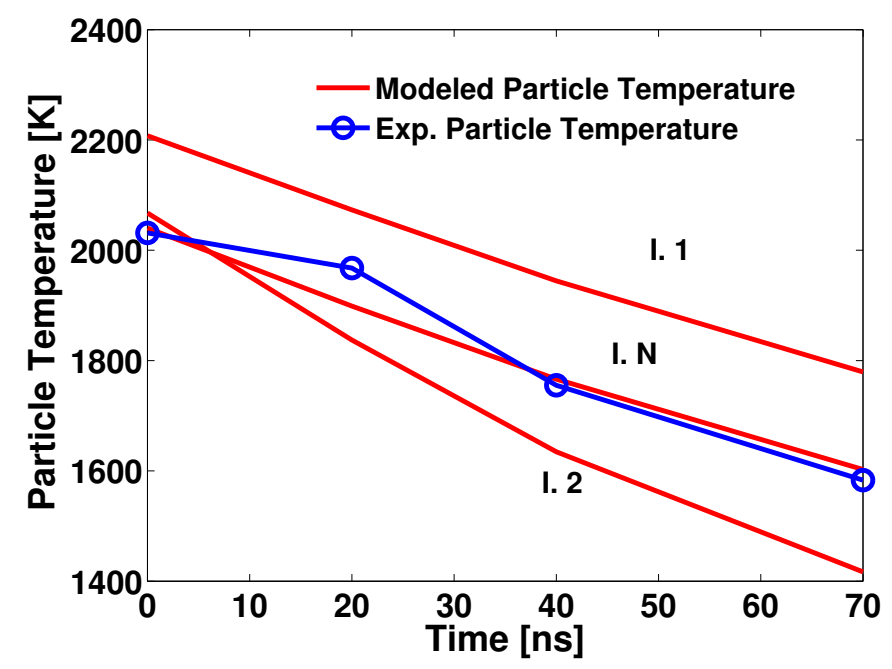

Figure 2.7: Example of iterative least-squares fitting of model and experimentally measured particle temperature decays.

\subsubsection{Soot Volume Fraction}

The generalized equation for volume fraction is a function of the number of primary soot particles per aggregate $\left(N_{p}\right)$, number density of aggregate particles $\left(N_{a g}\right)$ and primary particle diameter $\left(d_{p}\right)$ and is defined by Eq. 2.7 with the assumption of a particle diameter distribution and aggregate distribution,

$$
f_{v}=\frac{\pi N_{a g}}{6} \int_{0}^{\infty} \int_{1}^{\infty} N_{p} d_{p}^{3} \operatorname{pdf}\left(N_{p}\right) \operatorname{pdf}\left(d_{p}\right) d N_{p} d d_{p} .
$$

Physically, soot volume fraction represents the proportionality between the known radiance of soot at a measured temperature and the irradiance from a volume of soot in a total 
volume of space measured by the detector from the soot layer of a flame. As such, soot volume fraction is calculated from the measured peak incandescent signal at peak particle temperature (for best signal to noise) and the modeled spectral radiance of soot at the same temperature by Eq. 2.8 extended from Snelling et al. [61],

$$
f_{v, i}=\frac{I_{L I I, p}\left(\lambda_{i}\right) \pi \eta_{i}}{\Omega A_{e} I_{m, p}\left(\lambda_{i}\right)}
$$

$I_{L I I, p}\left(\lambda_{i}\right)$ is the average measured peak incandescent signal represented as counts for one pixel of the CCD camera at the filter wavelength $\lambda_{i}, \eta_{i}$ the calibration factor, $I_{m, p}\left(\lambda_{i}\right)$ the modeled peak incandescent signal from Eq. 2.4, $\Omega$ the measured solid angle of the detector optics, and $A_{e}$ the calibrated area of one pixel from a spatial calibration of the detection device in the configuration used to conduct LII experiments. In this study, the volume fraction measurement reported is the average from the two detection wavelengths, $f_{v}=\left(f_{v, 1}+f_{v, 2}\right) / 2$. However, proper choice of weight factors used in the primary particle diameter fitting routine results in good convergence between the two signal wavelengths and thus the measured volume fractions are nearly identical.

\subsubsection{Number Density}

The general form for total number density of primary soot particles in the local soot region is given by the physical definition,

$$
N=N_{a g} \int_{1}^{\infty} N_{p} \operatorname{pdf}\left(N_{p}\right) d N_{p} .
$$

Here, the number density $N$ is calculated using the best-fit primary particle size and assumed size distribution and the volume fraction from Eq. 2.8, yielding

$$
N=\frac{6 f_{v}}{\pi} /\left(\int_{0}^{\infty} d_{p}^{3} \operatorname{pdf}\left(d_{p}\right) d d_{p}\right)
$$




\subsubsection{Heat Transfer Model}

Accurate determination of LII extracted soot volume fraction $f_{v}$, primary particle diameter $d_{p}$, and number density $N$ requires a heat transfer model of the heat exchange between a particle and the surrounding gas. The details of the LII heat transfer model and solution procedure have been extensively documented in previous work [70,71]. Since many research groups have employed slightly different approaches to LII heat transfer modeling, we list below only a summary of the specific governing equations and modifications relevant to this study. An assumption of particle aggregation is allowed in this formulation and the common Rayleigh-Debye-Gans (RDG) approach is utilized as a modification to account for aggregation of primary soot particles. The heat transfer mechanisms controlling the temperature decay of soot particles are illustrated in Fig. 2.8. The governing equation

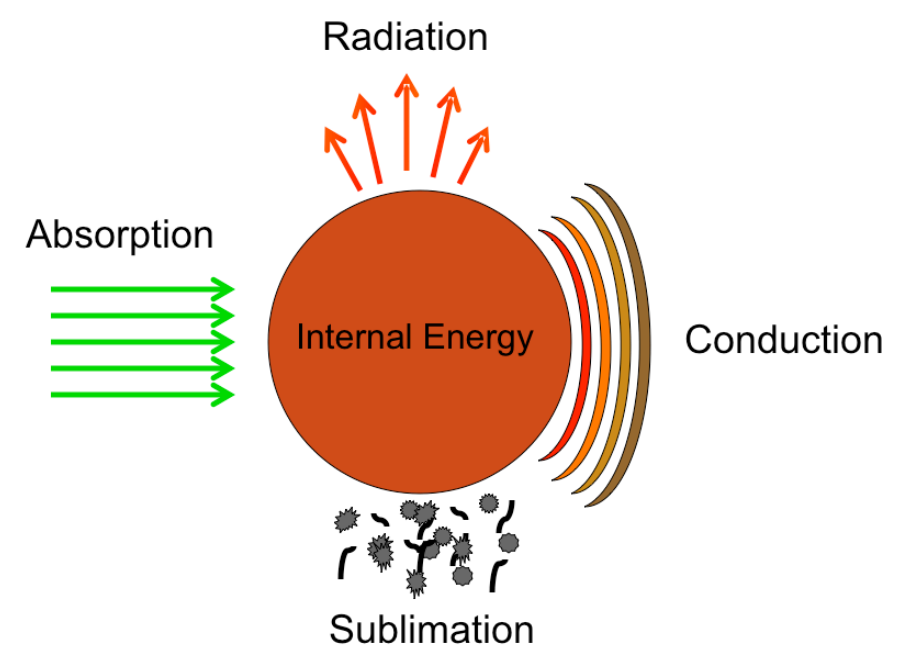

Figure 2.8: Illustration of the heat transfer mechanisms involved in the soot particle energy balance.

relating the rate of change of internal energy of a nano-sized primary soot particle is thus,

$$
\frac{d U_{\text {internal }}}{d t}=\dot{Q}_{\text {absorption }}-\dot{Q}_{\text {radiation }}-\dot{Q}_{\text {sublimation }}-\dot{Q}_{\text {conduction }} .
$$

Where the right hand side terms are the rates of pulsed laser energy absorbed by the soot particle and heat lost via conduction, radiation, and sublimation heat transfer mechanisms. 
Equation 2.11 can also be written in terms of the rate of change of particle temperature, size, and mass. In particular, the rate of change of internal energy of the particle can be written as,

$$
\frac{d U_{\text {internal }}}{d t}=\rho_{s} c_{s} \frac{\pi}{6} d_{p}^{3} N_{p} \frac{d T}{d t} .
$$

Where $\rho_{s}$ and $c_{s}$ are the density and specific heat of soot, $N_{p}$ the number of particles per aggregate where an aggregate or non-aggregated $\left(N_{p}=1\right)$ assumption can be made. The rate of pulsed laser energy absorbed by the particle is given by [90],

$$
\dot{Q}_{\text {absorption }}=\frac{\pi^{2} d_{p}^{3} E(m) N_{p}}{\lambda_{L}} \frac{F_{0} q(t)}{q_{1}}
$$

Where the Rayleigh approximation for volumetric absorption of light by small particles (given $\pi d_{p} / \lambda_{L}<0.3$ ) is assumed valid, $E(m)=-\operatorname{Im}\left[\frac{m^{2}-1}{m^{2}+2}\right]$ is the absorption function of soot at the laser wavelength $\lambda_{L}, F_{0}$ the laser fluence, and $q(t) / q_{1}$ the normalized laser temporal profile where integration over all time is equal to unity. A Gaussian temporal profile with a FWHM of $8 \mathrm{~ns}$ was assumed based on temporal profile measurements made on the laser used in this study. Light absorption by a soot particle is assumed here to exhibit a $1 / \lambda$ dependence in accordance with a majority of literature [72]. The rate of radiation heat transfer from Planck's law integrated over all wavelengths is given by the following equations.

$$
\begin{gathered}
\dot{Q}_{\text {radiation }}=\pi d_{p}^{2} N_{p} \int_{0}^{\infty} \epsilon_{\lambda} \frac{2 \pi h c^{2}}{\lambda^{5}\left[\exp \left(\frac{h c}{\lambda k_{B}\left(T-T_{0}\right)}\right)-1\right]} d \lambda \\
\epsilon_{\lambda}=4 \pi d_{p} E(m) / \lambda .
\end{gathered}
$$

The Rayleigh approximation for the emissivity $\epsilon_{\lambda}$ is assumed and is equal to the absorption efficiency under consideration of Kirchhoff's Law. Eq. 2.14 accounts for reabsorption of radiation at the local gas temperature $T_{0}$. The expression for the rate of heat loss due to 
sublimation is given by the following equations [70].

$$
\begin{gathered}
\dot{Q}_{\text {sublimation }}=-\frac{\Delta H_{v}}{W_{v}} \frac{d M}{d t} \\
\frac{d M}{d t}=\frac{-\pi d_{p}^{2} N_{p} W_{v} \alpha_{M} p_{v}}{R_{p} T} \sqrt{\frac{R_{m} T}{2 \pi W_{v}}} \\
p_{v}=p_{\text {ref }} \exp \left[-\frac{\Delta H_{v}}{R}\left(\frac{1}{T}-\frac{1}{T_{r e f}}\right)\right]
\end{gathered}
$$

$W_{v}$ is the molecular weight of sublimed carbon, $\Delta H_{v}$ the enthalpy of formation of sublimed carbon, $\alpha_{M}$ the mass accommodation coefficient, $p_{r e f}$ and $T_{r e f}$ the reference pressure and temperature, and $R, R_{p}$, and $R_{m}$ the universal gas constant expressed in different units. Conductive cooling of the soot particle to the surrounding gas is expressed by the Sherman model [73] in the transition regime,

$$
\begin{gathered}
\dot{Q}_{\text {conduction }}=\left[\frac{1}{\dot{Q}_{C}}+\frac{1}{\dot{Q}_{F M}}\right]^{-1}, \\
\dot{Q}_{C}=2 \pi D_{e f f} \kappa_{h}\left(T-T_{g}\right), \\
\dot{Q}_{F M}=\alpha_{T} \pi D_{\text {eff }}^{2} \frac{p_{0}}{8} \frac{\gamma_{h}+1}{\gamma_{h}-1} \sqrt{\frac{8 R_{m} T_{g}}{\pi W_{g}}}\left(\frac{T}{T_{g}}-1\right) .
\end{gathered}
$$

The validity and limitations of employing various conduction models are well documented (see [74] for an extensive review). $\dot{Q}_{C}$ is the continuum heat conduction rate, $\dot{Q}_{F M}$ is the free molecular heat conduction rate, $\kappa_{h}$ is the harmonic mean thermal conductivity, $\gamma_{h}$ the harmonic mean specific heat capacity ratio, $p_{0}$ the gas pressure, and $W_{g}$ the molecular weight of the gas. The expressions employ RDG theory to estimate an effective diameter $D_{\text {eff }}$ due to partial primary particle surface shielding from particle aggregation by Brasil et al. [75]. This formulation differs from that commonly applied in literature which uses a shielding relation based on 2D projected aggregate areas also proposed by Brasil et al. The original surface reduction relation adopted here has the added benefit of converging 
to the correct relation for particle diameter under the assumption of no aggregation i.e. $D_{e f f}=d_{p}$. The equation follows,

$$
D_{e f f}=d_{p} \sqrt{N_{p}\left[1-\phi C_{o v}\left(1-\frac{1}{N_{p}}\right)\right]} .
$$

The convention to mathematically represent fractal aggregate soot particles dimensions uses two parameters; the fractal prefactor and fractal dimension $k_{f}$ and $D_{f}[75,91,125]$. A typical value of $D_{f}=1.8$ is cited as common to many flame studies with generally little variation [75]. The fractal prefactor $k_{f}$ is more variable and dictates the extent of aggregate shielding through a primary particle overlap parameter,

$$
C_{o v}=-0.0735 k_{f}^{2}+0.5399 k_{f}-0.6398 .
$$

A fitting parameter $\phi$ (originally proposed as 1.3 by Brasil et al. [75]) was tuned to DSMC data for the effect of thermal accommodation on changes in aggregate surface shielding [76-78].

$$
\phi=1.7\left(0.7144 \alpha_{T}+0.2873\right)
$$

The effective diameter due to aggregate shielding is assumed equal for the continuum and free molecular expressions used in the Sherman formulation for transition regime heat conduction. The coupled differential equations for changes in soot particle mass and temperature are solved using a fourth-order Runge-Kutta method and a time step of $400 \mathrm{ps}$.

Local variation in gas temperature and variations in the specific heat ratio, thermal conductivity, and molecular weight due to species concentrations and gas temperature were calculated using the Smooke's counterflow code [69] with SERDP chemical kinetic model [68] or 56 species skeletal model at each spatial location along the axis of symmetry of the counterflow burner in an effort to best represent the spatially varying properties of the counterflow configuration. The skeletal model was obtained by applying the procedure 
in Esposito and Chelliah [79] to a C1-C4 baseline model from Sheen and Wang [80]. In the reduction procedure, global combustion properties of ethylene non-premixed extinction, flame propagation and ignition were all included. The temperature profiles calculated from SERDP, Sheen and Wang, and skeletal models at the strain rates of interest are indistinguishable. The molecular weight of species and the fitting parameters from the computational model for heat capacity and thermal conductivity as a function of temperature for each species were loaded and calculated in the LII model for temperatures from 300$4500 \mathrm{~K}$. The species mass fractions $Y_{k}$ at each spatial location were used to determine the mixture averaged molecular weight,

$$
W_{g}=\frac{1}{\sum_{k=1}^{K} Y_{k} / W_{k}} .
$$

The mixture averaged heat capacity and thermal conductivity were calculated at each spatial location over the temperature range of 300-4500 K. The equations for mixture averaged mole fraction, heat capacity, and thermal conductivity are given respectively and specified from Sandia Reports [81, 82],

$$
\begin{gathered}
X_{k}=Y_{k} \frac{W_{g}}{W_{k}}, \\
\bar{C}_{p}=\sum_{k=1}^{K} C_{p k} X_{k}, \\
\kappa=\frac{1}{2}\left(\sum_{k=1}^{K} X_{k} \kappa_{k}+\frac{1}{\sum_{k=1}^{K} X_{k} / \kappa_{k}}\right) .
\end{gathered}
$$

The heat capacity ratio can be calculated according to,

$$
\gamma=\frac{\bar{C}_{p}}{\bar{C}_{p}-R} .
$$

The thermal conductivity and heat capacity ratio as a function of temperature were fit to 2nd and 8th degree polynomials respectively and passed to the LII model to minimize processing time. The unique aspect of this work utilizes harmonic mean heat transfer variables 
for thermal conductivity $\kappa_{h}$ and specific heat capacity ratio $\gamma_{h}$ evaluated at local gas temperature and particle temperature. The exact formulation for all relevant LII variables are listed in Table 2.1.

Table 2.1: Parameters for heat transfer model

\begin{tabular}{ccc}
\hline \hline & Parameter & Description \\
\hline Internal & $\rho_{s}$ & $2.3031-7.3106 * 10^{-5} \mathrm{~T}[83]$ \\
& $c_{s}$ & T-dependent $[83,84]$ \\
\hline Absorption and Radiation & $E(m)$ & $\sim 0.24$ or allowed to vary \\
& $F$ & $0.098-0.111 \mathrm{~J} / \mathrm{cm}^{2}$ peak \\
\hline Sublimation & $p_{v}$ & Clausius-Clapeyron Eq. [70,85] \\
& $H_{v}$ & T-dependent $[70,85]$ \\
& $W_{v}$ & T-dependent $[70,85]$ \\
& $\alpha_{m}$ & $0.8[70]$ \\
\hline Conduction & $\alpha_{T}$ & $0.3[70]$ or local mixture averaged \\
& $\gamma_{h}$ & $2 /\left(\frac{1}{\gamma_{T_{g}}}+\frac{1}{\gamma_{T}}\right)$ \\
& $\kappa_{h}$ & $2 /\left(\frac{1}{\kappa_{T_{g}}}+\frac{1}{\kappa_{T}}\right)$ \\
$W_{g}$ & Local mixture averaged \\
& $D_{f}$ & $1.8[75]$ or calculated via TEM \\
$k_{f}$ & Calculated via TEM \\
\hline
\end{tabular}

\subsubsection{Heat Transfer Model Validation}

Liu et al. [74] recommended the use of Fuchs boundary sphere model with integrated mean heat transfer properties as a best fit to DSMC calculations of conduction heat transfer from a spherical particle. This approach requires iteration however, where a simpler non-iterative approach was desirable in this work. Two heat transfer formulations, the McCoy and Cha [86] and Sherman [73] models match the requirement of simply implementation without iteration. Figure 2.9 shows the performance of several heat conduction models in terms of Nusselt number as a function of Knudsen number and pressure analyzed by Liu et al. [74] including DSMC results for a particle temperature of $3400 \mathrm{~K}$, gas temperature of $300 \mathrm{~K}$, and particle size of $30 \mathrm{~nm}$. The Nusselt number corresponding to heat transfer was normalized by half the continuum heat transfer result with thermal conductivity of the surrounding gas 
evaluated at an ambient gas temperature of $300 \mathrm{~K}$. The experimental limit of pressure in this work is 30 atmospheres represented by the black vertical line in Fig. 2.9. Clearly the Fuchs approach suggested by Liu et al. and the Sherman approach with harmonic heat conduction properties are shown as equally valid for pressures up to 30 atm and only show significant variation at several hundred atmospheres. Most notably, the McCoy and Cha model exhibits persistent under-prediction of the particle heat transfer rate over all pressures and was therefore ruled out. Thus the harmonic Sherman approach was utilized in this work as an acceptable alternative to Fuchs formulation.

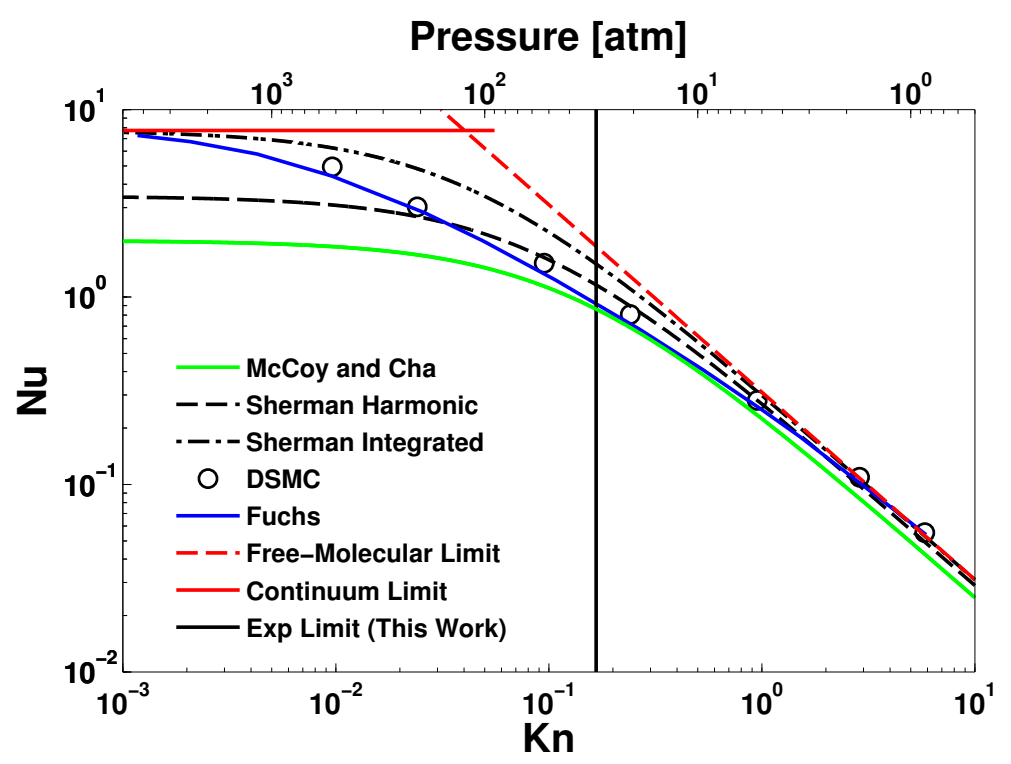

Figure 2.9: Nusselt numbers calculated from several heat conduction models including DSMC results by Liu et al. [74] for a range of Knudsen numbers and pressures at a gas temperature of $300 \mathrm{~K}$.

Additional validations were conducted on the heat transfer model based on a comparison of models in literature using both a fully constrained baseline model and an independently developed model with semi-constrained conditions [70]. Both model comparisons utilized fixed initial conditions assuming air at an ambient pressure of 1 bar, ambient temperature of $1800 \mathrm{~K}$, primary particle size of $30 \mathrm{~nm}$, a spatially uniform laser fluence of 0.05 $\mathrm{J} / \mathrm{cm}^{2}$ at $532 \mathrm{~nm}$ with a numerically defined temporal profile, and an incandescent signal represented at $500 \mathrm{~nm}$ integrated over the entire particle surface. The fluence used in this 


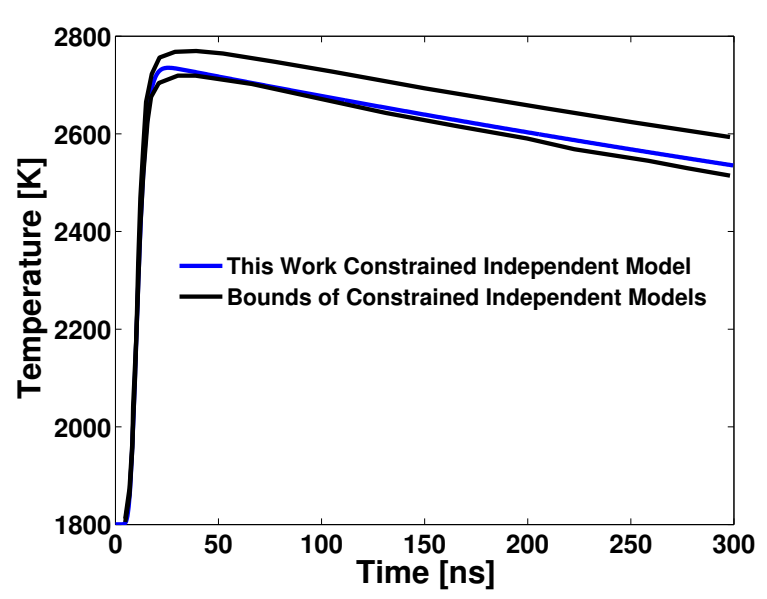

(a) Temperature

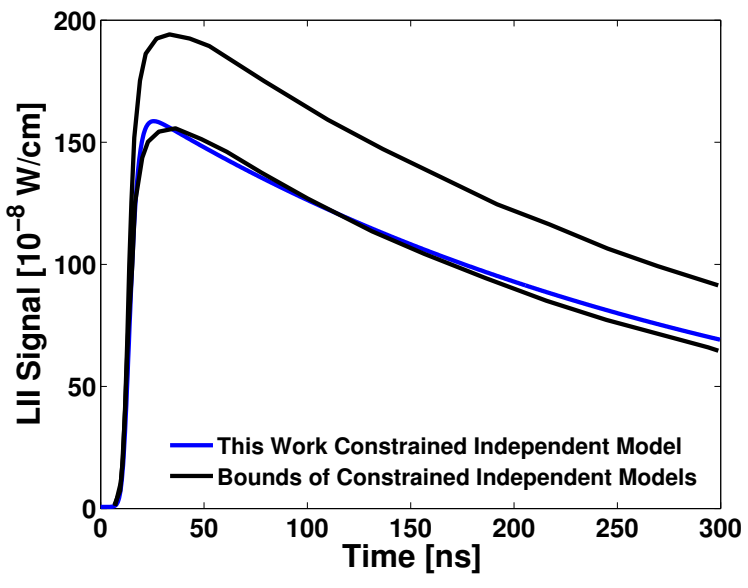

(b) LII Signal

Figure 2.10: Comparison of modeled a) temperature and b) LII signal decay from the semiconstrained independent models.

comparison is well within the low fluence regime where sublimation of carbon from the particle surface is avoided. Heat loss by radiation is also negligible in all but sub-atmospheric conditions. Thus heat loss by the particle in these comparisons is dominated by conduction. Fixed heat transfer expressions and model variables were used for the fully constrained model comparison and resulted in identical temporal decays of particle temperature and incandescent signal. The numerical laser temporal profile used in the workshop [70] was not available so a lognormal temporal profile was applied to the heat transfer model developed in this work. This resulted in a slightly steeper temperature rise near peak temperature during laser heating and is the only cause of variation in the fully constrained comparison. The second comparison utilized heat transfer models with heat transfer mechanisms independently developed by the research groups. Heat transfer variables $\rho_{s}, c_{s}, E(m), W_{g}$, $\alpha_{T}$, and $\alpha_{M}$ remained constrained while all other model variables were specified independently, the details of which can be found in the review paper by Michelsen et al. [70]. By constraining the most influential model variables, this comparison served to illustrate differences in conduction heat transfer mechanisms applied by the different research groups. Figure 2.10 shows the temporal temperature and incandescent signal decays for the semiconstrained model used in this work and the maximum and minimum bounds from the sam- 
ple of independent semi-constrained models reported in the summary article. The results of the model used in this work closely match that of Liu and Hoffmann near the minimum bound [70]. The agreement is due to the use of a conduction model formulation quantitatively similar to the iterative Fuchs boundary sphere method used by Liu and Hoffman. Additionally, average heat transfer properties $\gamma$ and $\kappa$ between the particle temperature and ambient gas temperature are applied to both approaches where harmonic mean properties

(i.e. $2 /\left(\frac{1}{\gamma_{T_{g}}}+\frac{1}{\gamma_{T}}\right), 2 /\left(\frac{1}{\kappa_{T g}}+\frac{1}{\kappa_{T}}\right)$ ) are used here and integrated mean properties are utilized in the Liu and Hoffmann formulations. This comparison serves to validate the low fluence LII heat transfer model used in this work under constrained parameters where model variables can then be freely optimized for specific LII experimental applications. The comparison also serves to validate soot primary particle sizing based on particle temperature decay. A baseline agreement in particle temperature decay between models in literature indicates all models would perform similarly when extracting primary soot particle sizes under similar experimental conditions and assumptions. Validation with other measurement techniques on the high pressure counterflow flame geometry is not possible due to spatial constraints on rapidly varying flame properties. However, numerous validations and comparisons to other measurement techniques have been conducted in literature on other burner configurations over a range of conditions with acceptable agreement $[24,87,88]$. This indicates the LII model developed in this study is acceptable for determining soot primary particle sizes within a prescribed uncertainty bound.

\subsection{Elastic Light Scattering Aggregate Submodel}

A model of elastic light scattering (ELS) was implemented to explore aggregate properties of soot if they were thought to exist in any counterflow flame experiments. The method described by Sorensen [30] and combined with LII by Reimann et al. [89] is adopted here. The treatment is the same as individual particle scattering from Bohren and Huffman [90] 
with an aggregate structure factor $S\left(q R_{g}\right)$ included. The scattering intensity $I_{s c a}$ per solid angle is expressed as

$$
I_{s c a}=I_{i n c} N_{a g} N_{p}^{2} \frac{d \sigma^{p}}{d \Omega} S\left(q R_{g}\right)
$$

The differential scattering cross section of a single primary particle is given by [90]

$$
\frac{d \sigma^{p}}{d \Omega}=\left(\frac{2 \pi}{\lambda}\right)^{4}\left(\frac{d_{p}}{2}\right)^{6}\left|\frac{m^{2}-1}{m^{2}+2}\right|^{2}
$$

where $I_{i n c}$ is the incident laser intensity, $N_{a g}$ the number density of aggregates in the probe volume, $N_{p}$ the number of primary particles in an aggregate where $N_{p} N_{a g}=N$, the scattering wave vector $q=\frac{4 \pi}{\lambda} \sin (\theta / 2)$, and $R_{g}$ the radius of gyration of the aggregate, $\lambda$ the laser wavelength, $\theta$ the scattering angle between the detector and laser, $d_{p}$ the primary particle diameter, and $m$ the complex index of refraction of soot. The number of primary particles in an aggregate and the radius of gyration of a fractal aggregate are related by the expression $[75,91,125]$

$$
N_{p}=k_{f}\left(\frac{2 R_{g}}{d_{p}}\right)^{D_{f}}
$$

where $D_{f}$ and $k_{f}$ are the fractal dimension and fractal prefactor representing the fractal aggregate dimensions. Assuming arbitrary distributions for primary particle diameter, $\operatorname{pdf}\left(d_{p}\right)$, and the number of particles per aggregate, $\operatorname{pdf}\left(N_{p}\right)$, the expression for scattering intensity becomes

$$
\begin{aligned}
& I_{s c a}=I_{i n c} N_{a g}\left(\frac{2 \pi}{\lambda}\right)^{4}\left|\frac{m^{2}-1}{m^{2}+2}\right|^{2} \\
& \int_{0}^{\infty} \int_{1}^{\infty} N_{p}^{2}\left(\frac{d_{p}}{2}\right)^{6} S\left(q R_{g}\left(N_{p}, d_{p}\right)\right) \operatorname{pdf}\left(N_{p}\right) \operatorname{pdf}\left(d_{p}\right) d N_{p} d d_{p} .
\end{aligned}
$$


Dividing Eq. 2.33 by the volume fraction of soot $f_{v}$ in the probe volume $V_{p}$ cancels the parameter $N_{a g}$ and results in

$$
\begin{aligned}
\frac{I_{s c a}}{f_{v}}=V_{p} I_{i n c}\left(\frac{2 \pi}{\lambda}\right)^{4}\left|\frac{m^{2}-1}{m^{2}+2}\right|^{2} & \frac{\int_{0}^{\infty} \int_{1}^{\infty} N_{p}^{2}\left(\frac{d_{p}}{2}\right)^{6} S\left(q R_{g}\left(N_{p}, d_{p}\right)\right) \operatorname{pdf}\left(N_{p}\right) \operatorname{pdf}\left(d_{p}\right) d N_{p} d d_{p}}{\frac{\pi}{6} \int_{0}^{\infty} \int_{1}^{\infty} N_{p} d_{p}^{3} \operatorname{pdf}\left(N_{p}\right) \operatorname{pdf}\left(d_{p}\right) d N_{p} d d_{p}}
\end{aligned}
$$

Two unknown parameters remain in Eq. 2.34 and are cancelled out by performing a calibration of scattering intensity from a pure gas with identical operating conditions to the soot scattering measurement. Nitrogen was chosen here for its relative availability and well known scattering properties. The expression governing the scattering intensity from nitrogen is

$$
I_{s c a, c}=I_{i n c} N_{N_{2}} V_{p} \frac{d \sigma_{N_{2}}}{d \Omega}
$$

The same incident laser intensity $I_{i n c}$ and probe volume $V_{p}$ are used as before. The number density of nitrogen molecules $N_{N_{2}}$ can be determined from the ideal gas law. The differential scattering cross section of nitrogen is determined from the total scattering cross section $\sigma_{N_{2}}=5.3 \times 10^{-27} \mathrm{~cm}^{2}$ at $532 \mathrm{~nm}$ laser wavelength by $\frac{d \sigma_{N_{2}}}{d \Omega}=\frac{3}{8 \pi} \sigma_{N_{2}}$ [92]. Dividing Eq. 2.34 by Eq. 2.35 cancels the remaining unknown variables $I_{i n c}$ and $V_{p}$ and results in the expression

$$
\begin{aligned}
\frac{I_{s c a}}{I_{s c a, c} f_{v}}=\left(\frac{2 \pi}{\lambda}\right)^{4} & \left|\frac{m^{2}-1}{m^{2}+2}\right|^{2} \\
& \frac{\int_{0}^{\infty} \int_{1}^{\infty} N_{p}^{2}\left(\frac{d_{p}}{2}\right)^{6} S\left(q R_{g}\left(N_{p}, d_{p}\right)\right) \operatorname{pdf}\left(N_{p}\right) \operatorname{pdf}\left(d_{p}\right) d N_{p} d d_{p}}{N_{N_{2}} \frac{d \sigma_{N_{2}}}{d \Omega} \frac{\pi}{6} \int_{0}^{\infty} \int_{1}^{\infty} N_{p} d_{p}^{3} \operatorname{pdf}\left(N_{p}\right) \operatorname{pdf}\left(d_{p}\right) d N_{p} d d_{p}}
\end{aligned}
$$


When simplified under the assumption of monodisperse primary particle diameter $\left(d_{p}\right)$ and number of primary particles per aggregate $\left(N_{p}\right)$, Eq. 2.36 reduces to the following equation

$$
\frac{I_{s c a}}{I_{s c a, c} f_{v}}=\frac{1}{N_{N_{2}} \frac{d \sigma_{N_{2}}}{d \Omega}} \frac{3 \pi^{3}}{2 \lambda^{4}} d_{p}^{3} N_{p}\left|\frac{m^{2}-1}{m^{2}+2}\right|^{2} S\left(q R_{g}\right) .
$$

Differential scattering cross sections can be used in place of full scattering cross sections without loss of accuracy due to the very small solid angle of the detector. Integrating the scattering expressions for changes in scattering intensity over the detector solid angle are negligible. Finally the structure factor is given based on the aggregate size regime,

$$
S\left(q R_{g}\right)=\left\{\begin{aligned}
1-q^{2} R_{g}^{2} / 3, & \mathrm{qR} R_{\mathrm{g}} \leq 1 \\
F_{1}\left[D_{f} / 2,3 / 2 ;-\left(q R_{g}\right)^{2} / D_{f}\right], & \mathrm{qR}_{\mathrm{g}}>1
\end{aligned}\right.
$$

The scalable dimension $q R_{g}$ determines the treatment of the structure factor. Under the limit of $q R_{g} \leq 1$ a simple approximate solution is given by $S\left(q R_{g}\right)=1-q^{2} R_{g}^{2} / 3$ [30]. As the radius of gyration increases and the size of the aggregate increases past the point of $q R_{g}>1$, the scattering intensity becomes less and less dependent on the aggregate size. The most well accepted cutoff function to represent the structure factor in this regime is given by a Gaussian cutoff with the solution given by Kummers hypergeometric function [30]. In general it is advisable to remain near or below the limit $q R_{g} \leq 1$ corresponding to an aggregate radius of gyration limit of approximately $60 \mathrm{~nm}$. The Eqs. 2.36 or 2.37 and 2.38 allow for least squares fitting of either the number of primary particles per aggregate, or the radius of gyration of the aggregate when the volume fraction and primary particle size is determined from LII using Rayleigh-Debye-Gans theory and a fractal aggregate model of soot particle heat transfer. With a scattering submodel considered, the volume fraction independent of particle and aggregate size is first solved. The particle size and aggregate size are then solved iteratively until the particle temperature decay from LII and the scattering intensity from ELS reach a converged solution where the combined squared 
error of both models is minimized.

\subsection{Extinction Correction}

Upon close inspection of the two dimensional intensity profile captured during LII experiments of sooting flames, it is clear that the measured LII signal exhibits a gradient in the radial direction, evidence of signal trapping across the soot layer. This gradient was most noticeable at higher soot concentrations and clearly identified a need to correct for errors due to signal trapping from extinction. The adopted correction procedure is similar to an LII extinction calibration and correction procedure by Choi and Jensen [93] for axisymmetric flames. The original work proposed an iterative technique to calibrate and correct LII data through a best-fit solution between a line of sight extinction measurement and LII signal under the assumption of a single calibration factor between LII signal and line of sight extinction. This assumption is considered relevant in the region of high-fluence LII where the LII signal plateaus once all particles have reached vaporization temperature. However, Choi and Jensen assumed no extinction of the laser fluence affected the high fluence assumption. The chance to infer particle size from the temporal signal decay was also lost due to large uncertainties in the vaporization regime of all nano-scale soot particle heat transfer models. The geometrical construct from Choi and Jensen is relevant to this work however, with additional considerations given to low-fluence LII. The geometry is shown in Fig. 2.11. First the calibrated laser fluence $F_{0}$ is corrected to the fluence expected after extinction through the soot layer by a path length from $x(1)=-R_{f}$ to a point of interest $x(i)$ given by the equation,

$$
F_{\text {ext }}(i)=F_{0}(i) \prod_{i} T_{i}
$$




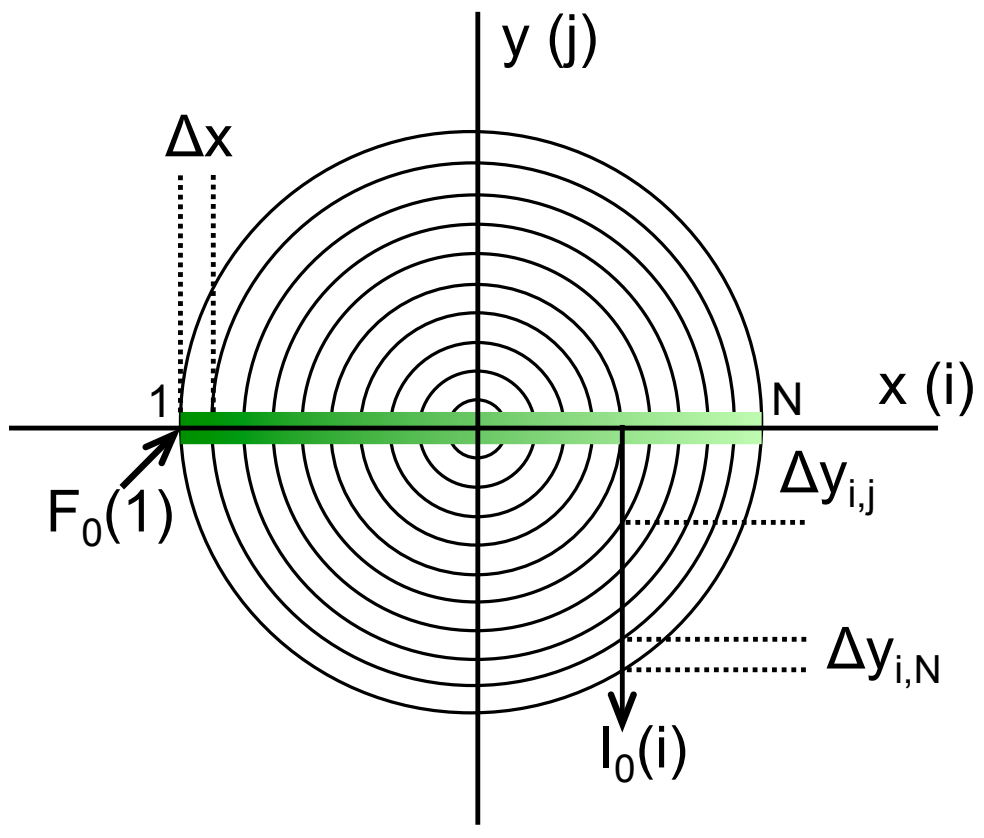

Figure 2.11: Flame geometry with the laser entering along the $x$-axis from the left at $y=0$. The LII signal is collected in the $y$-dimension after signal trapping. The soot region is divided into rings on the $x-y$ plane corresponding to pixels of LII data. The LII signal also varies in the axial or $z$-dimension in and out of the page.

The total fluence transmission is calculated by the product of signal transmission through each pixel shell defined by the Beer-Lambert law

$$
T_{i}=\exp \left[-f_{v}(i)\left(c_{a b s}(i)+c_{s c a}(i)\right) \Delta x_{i}\right]
$$

Where $f_{v}(i)$ is the volume fraction in the $i$ th shell, $\Delta x_{i}$ is the length of the $i$-th shell, and $c_{a b s}(i)$ and $c_{s c a}(i)$ are the specific particle volume absorption and scattering cross sections in the $i$-th shell respectively. The relations for $c_{a b s}(i)$ and $c_{s c a}(i)$ from Bohren and Huffman [90] are given by

$$
c_{a b s}(i)=\frac{C_{a b s}(i)}{V}=\frac{6 \pi E(m)_{i}}{\lambda},
$$




$$
\begin{aligned}
c_{\text {sca }}(i)=\frac{C_{\text {sca }}(i)}{V} & \\
& =\frac{4 \pi^{4} F(m) \int_{0}^{\infty} \int_{1}^{\infty} N_{p}^{2} d_{p}^{6} S\left(q R_{g}\left(N_{p}, d_{p}\right)\right) \operatorname{pdf}\left(N_{p}\right) \operatorname{pdf}\left(d_{p}\right) d N_{p} d d_{p}}{\lambda^{4} \int_{0}^{\infty} \int_{1}^{\infty} N_{p} d_{p}^{3} \operatorname{pdf}\left(N_{p}\right) \operatorname{pdf}\left(d_{p}\right) d N_{p} d d_{p}} .
\end{aligned}
$$

In the realm of highly absorbing small particles in the Rayleigh limit as is the case here, scattering is negligible but is included for rigor. An arbitrary assumption of a particle diameter distribution is also included. The laser wavelength is represented by $\lambda$ and the absorption and scattering functions, $E(m)=-\operatorname{Im}\left[\frac{m^{2}-1}{m^{2}+2}\right]$ and $F(m)=\left|\frac{m^{2}-1}{m^{2}+2}\right|^{2}$, are related to the complex index of refraction $m$ of soot. The unknown variable $F(m)$ is assumed 0.2 as an average from literature [72]. The effect of $F(m)$ on extinction correction results is generally negligible. The unknown variable $E(m)$ can be assumed from literature $[70,72]$ or iteratively solved for as described in Chapter 3.1.4. Given a monodisperse particle and aggregate assumption

$$
c_{s c a}(i)=\frac{C_{s c a}(i)}{V}=\frac{4 \pi^{4} F(m) N_{p} d_{p}^{3} S\left(q R_{g}\left(N_{p}, d_{p}\right)\right)}{\lambda^{4}} .
$$

Under the assumption of no aggregation, $S\left(q R_{g}\right)=1$ and $N_{p}=1$. After correcting for the remaining laser fluence transmitted to a point $x(i)$, the two incandescent signal profiles measured at $450 \mathrm{~nm}$ and $650 \mathrm{~nm}$ wavelengths are corrected for signal trapping in the $y$ dimension. Signal trapping is removed by the equation

$$
I_{L I I, e x t}(i)=I_{L I I, 0}(i) \prod_{j} \frac{1}{T_{i, j}}
$$

Where $I_{L I I, 0}(i)$ is the signal measured by the detector and $I_{L I I, \text { ext }}(i)$ is the signal measured by the detector if no signal trapping had occurred (corrected for extinction). The product of the signal transmission through each pixel shell given by

$$
T_{i, j}=\exp \left[-f_{v}(j)\left(c_{a b s}(j)+c_{s c a}(j)\right) \Delta y_{i, j}\right]
$$


Where the distance between two pixels in the $y$-dimension corresponding to a differential extinction length is given by

$$
\Delta y_{i, j}=\left\{\begin{array}{l}
\sqrt{x_{j}^{2}-x_{i}^{2}}-\sqrt{x_{j+1}^{2}-x_{i}^{2}} \quad \mathrm{x}_{\mathrm{i}}<0, \mathrm{j}=1: \mathrm{i}-1 \\
\sqrt{x_{j}^{2}-x_{i}^{2}}-\sqrt{x_{j-1}^{2}-x_{i}^{2}} \quad \mathrm{x}_{\mathrm{i}} \geq 0, \mathrm{j}=\mathrm{i}+1: \mathrm{N} .
\end{array}\right.
$$

If scattering is used to quantitfy aggregation, the scattering signal must also be corrected for extinction. In order to compare to the scattering calibration, the same laser intensity $I_{\text {inc }}$ must be used between calibration and experiment. Therefore the scattering signal is corrected for the loss of laser intensity and for signal trapping by the equation

$$
I_{s c a, e x t}(i)=I_{s c a, 0}(i) \prod_{i} \frac{1}{T_{i}} \prod_{j} \frac{1}{T_{i, j}}
$$

Where $I_{s c a, 0}(i)$ is the signal measured by the detector and $I_{\text {sca,ext }}(i)$ is the signal measured by the detector if no signal trapping had occurred.

A summary of the extinction correction procedure is as follows. The temporal LII intensity profile is loaded and particle peak and decay temperatures are calculated. The data is least squares fit to a model solution for particle diameter, soot volume fraction, and scattering if applicable without an extinction correction on the first iteration as an initial estimate. Equations 2.39-2.47 are applied to correct the laser fluence and LII and scattering signals. The corrected results are recalculated and corrected until convergence. Generally good convergence is observed on the third iteration. Equal $f_{v}, d_{p}$, and $N$ is assumed in the $x-y$ plane for extinction correction of one-dimensional LII measurements along the axial centerline of the counterflow burner. A comparison to two-dimensional results confirmed the assumption was acceptable. If two-dimensonal data is processed, results for $f_{v}, d_{p}$, and $N$ are used corresponding in the $y$-dimension to the same shell left or right of the centerline in the $x$-dimension in Fig. 2.11. 


\subsection{Thermophoretic Sampling Probe}

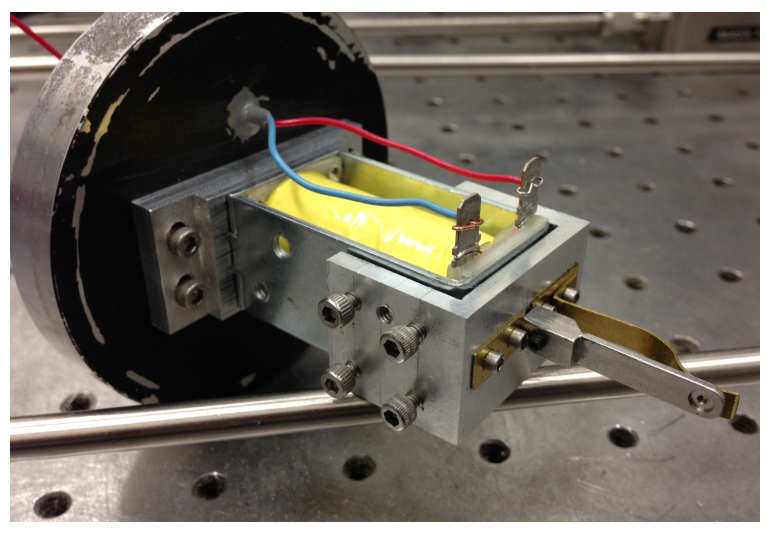

(a) Probing device

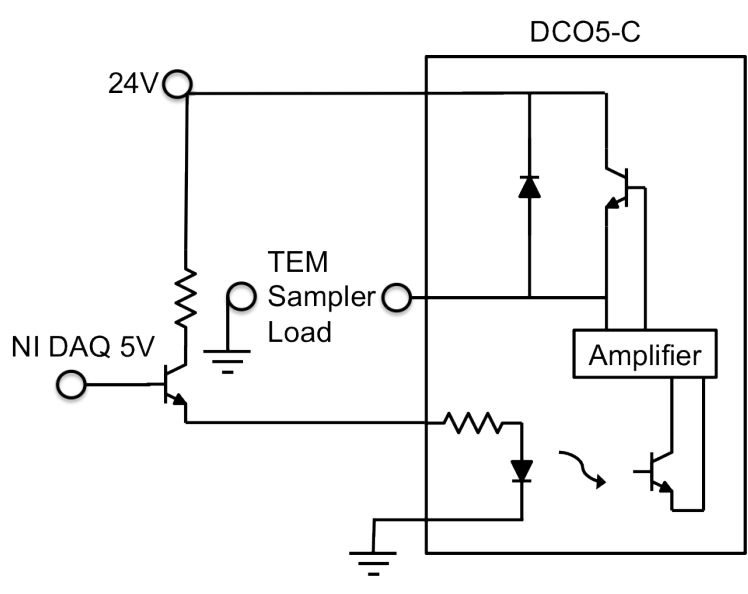

(b) Electrical circuit

Figure 2.12: a) Mechanical device and b) electrical circuit constructed for thermophoretic soot sampling on TEM grids.

A thermophoretic sampling probe was built based on optimized specifications by Lee et al. [94] to minimize vibrations and influence on the flame during actuation for Transmission Electron Microscopy (TEM) analysis of soot samples. High-pressure soot collection poses a challenge to pneumatically actuated systems and hence a linear solenoid was chosen as the actuating mechanism. Advantages of using solenoids for linear motion include reliability and ease of use at high pressure and a fast acting, well described, and electronically programmable actuation time. The circuit and mechanical device are shown in Fig. 2.12. The thermophoretic sampling probe is $1 \mathrm{~mm}$ thick and $4 \mathrm{~mm}$ tall to accommodate a $0.5 \mathrm{~mm}$ deep $3.1 \mathrm{~mm}$ diameter depression at the tip for placing a TEM grid (Electron Microscopy Sciences $\mathrm{CF} 400-\mathrm{Cu}$ ). The probe was filed to a knife-edge on all sides to minimize flame disturbance. The side with the depression is sealed during experimentation, and a $1 \mathrm{~mm}$ hole on the other side of the probe is used to thermophoretically deposit soot samples onto the TEM grid. An end of the probe is attached to the solenoid rod and the solenoid housing is attached to an adjustable mounting bracket so the center of the flame can be targeted with the probe height parallel to the flow. The entire system is fixed to an aluminum blank 
for placement in the high pressure chamber. A thin metal shield seals the thermophoretic sampling hole until the solenoid is actuated fully into the flame. The actuation time of the solenoid is specified and controlled in LabVIEW. The time response for full actuation of the solenoid is approximately $25 \mathrm{~ms}$. A sampling time or hold time at full actuation of approximately $100 \mathrm{~ms}$ was chosen based on work by Dobbins and Megaridis [95]. A twostage transistor circuit (2N3904 and Omega Engineering DC05-C) is used to amplify the NI DAQ current to a level large enough to actuate the solenoid. 


\section{Chapter 3}

\section{Uncertainty Analysis}

\subsection{Uncertainty}

\subsubsection{Detailed LII Model Parameter Uncertainty}

A concerted effort was undertaken to assess all sources and magnitudes of uncertainty from both measured experimental variables and assumed model variables. Details of each uncertainty are as follows.

$I_{L I I}$

Uncertainty of attenuation of the laser induced incandescence signal $\left(I_{L I I}\right)$ across the detector path due to suspended soot in the enclosed high-pressure chamber was calculated with a Thorlabs BC106-VIS beam profiler. An attenuated beam spanning the chamber due to soot absorption and scattering was compared to a reference beam under highly sooting conditions. The standard uncertainty estimate used here was a measured signal attenuation of $7 \%$ for highly sooting conditions. Soot coating the detector window was found negligible for individual datasets due to a relatively short data collection time. Windows were cleaned between datasets to eliminate soot buildup over time. 
The detector incandescent signal calibration factor $(\eta)$ uncertainty was calculated via error propagation of all independent uncertainties involved in the detector calibration. Measurement uncertainties due to distance (1.6\%), spectral irradiance $(2.5 \%)$, and CCD pixel area to image area calibration (2\%) were combined into a total measurement uncertainty by the square root of the sum squared error. A standard deviation repeatability uncertainty of $(5 \%)$ between the three calibration sets conducted was then error propagated with the total measurement uncertainty resulting in a final detector calibration factor standard uncertainty of $6 \%$.

$\Omega$

The uncertainty of the detector solid angle $(\Omega)$ measurement was estimated based on the repeatability in the distance measure between the detector and the soot signal due to the difficult geometry and arrangement of the equipment. The resulting standard uncertainty estimate was $2 \%$. The uncertainty in the measurement itself was negligible compared to repeatability due to the use of calipers.

$A_{e}$

Uncertainty of the CCD pixel area to image area calibration $\left(A_{e}\right)$ was calculated from the repeatability standard deviation of the calibration procedure for image to world mapping based on the known size of the counterflow burner nozzles in calibration images. The analysis resulted in a $2 \%$ uncertainty.

$T_{0}$

The local gas temperature $\left(T_{0}\right)$ profile is calculated from a quasi one-dimensional flowfield model coupled to a chemical kinetic model tuned for aromatic precursors and laminar flames for a set of conditions identical to each experimental sooting flame dataset [67-69]. The uncertainty arises from the matching of the modeled local gas temperature profile to the experimental soot incandescent profile and is based 
on a spatial uncertainty of the experimental flame location. Flow meter errors cause slight momentum changes in the opposed fuel and air streams of the counterflow burner resulting in small deviations of the flame location from the modeled case. Calibration and consistency of a zero image reference point relative to the exit of the air nozzle also plays a role in the estimated uncertainty of the local gas temperature. Large temperature gradients near the flame region are evident for all cases and increase with pressure and strain rate. The flame thickness controlling the temperature gradients can be shown to scale with the inverse square root of Reynolds number. This flame thickness scaling is convenient given the experimental requirement for a laminar flame, hence pinning the Reynolds number and flame thickness extremum. With the flame thickness minimum or temperature gradient maximum identified an estimate on the standard uncertainty of the local gas temperature due to spatial uncertainty was calculated as $250 \mathrm{~K}$. It is worth mentioning that as pressure is increased, additional uncertainty in modeled data is expected due to uncertainty in chemical reaction rates and third body efficiencies. However, these modeled uncertainties are largely unknown and difficult to quantify and hence are beyond the scope of this work. Radiation effects on the local gas temperature from large quantities of soot is also neglected but is shown to be at times significant under high soot loads and very low strain rates [96]. It is assumed that the uncertainty estimate due to spatial model to experimental matching is sufficient to appropriately represent the uncertainty in the local gas temperature.

The standard uncertainty in the local gas heat capacity ratio $(\gamma)$ is calculated based on the same spatial uncertainty method applied to the local gas temperature and resulted in a 0.014 uncertainty estimate. 
The standard uncertainty in the local gas thermal conductivity $(\kappa)$ is calculated based on the same spatial uncertainty method applied to the local gas temperature and resulted in a $0.0006 \mathrm{~W} / \mathrm{cmK}$ estimate.

$W_{g}$

The standard uncertainty in the local gas molar weight $\left(W_{g}\right)$ is calculated based on the same spatial uncertainty method applied to the local gas temperature and resulted in a $0.5 \mathrm{~g} / \mathrm{mol}$ estimate.

$y$

Uncertainty in the laser sheet spatial width $(y)$ was negligible after completion of a beam profile calibration.

$F_{0}$

The uncertainty of the laser fluence profile $\left(F_{0}\right)$ was determined by propagation of all independent error sources from a beam profile calibration using a Thorlabs BC106VIS beam profiler with a beam energy transfer calibration from a Scientech Astral Calorimeter. Shot to shot noise from the laser source was significant and on the order of $10 \%$ so all calibrations were conducted with 25 shot averages, the maximum allowable by the Thorlabs beam profiler. Uncertainties relevant to the calibration procedure were a $3 \%$ beam energy transfer calibration uncertainty and a $2.5 \%$ standard uncertainty due to beam energy drift inherent in successive 25 shot averages due to shot to shot noise. The square root of the sum-squared error resulted in $4 \%$ uncertainty. LII data samples are collected over 500 shot averages where the shot to shot noise becomes negligible and should tend toward the mean calibrated value. An additional uncertainty of $7 \%$ was considered due to laser fluence attenuation under highly sooting conditions. The result for the total sum squared standard uncertainty was $8 \%$. If no laser attenuation were present from soot particles along the line of sight, uncertainty in the fluence would be reduced to $4 \%$. 
Combustion chamber ambient pressure $\left(p_{0}\right)$ uncertainty was determined based on the average observable drift in the ambient pressure from several Omega PX409 series pressure transducers while experiments were conducted. The observable half width of approximately 0.06 atmospheres or less resulted in a standard uncertainty estimate $s=0.5 \cdot$ width $/ \sqrt{3}$ due to drift of 0.035 atmospheres based on a uniform distribution.

$\alpha_{T}$

In the case of soot particle heat transfer model input parameters, uncertainty in the heat conduction thermal accommodation coefficient $\left(\alpha_{T}\right)$ is significant and plays a large role in the uncertainty of the measured soot primary particle size. The standard strategy of the LII community is to apply one global value for thermal accommodation based on some form of experimentally or computationally derived data. The thermal accommodation coefficient represents the percentage of molecules that thermally accommodate upon collision with the laser heated soot particle, the rest undergoing specular reflection. When considering molecular geometry it is clear that the thermal accommodation coefficient is a function of the local gas composition as demonstrated using Direct Simulation Monte Carlo [97,98]. Many studies have utilized accommodation coefficient data from various molecular scattering experiments on carbon surfaces at temperatures of 700-1400 K [99, 100]. Questions arise however, on the validity of utilizing these results on the much higher temperature conditions relevant to laser induced incandescence of soot, most notably the potential effect of molecular dissociation or the influence of internal degrees of freedom. Other studies have attempted to determine the thermal accommodation coefficient by model fitting a measured soot particle temperature decay utilizing a well described sooting flame $[83,101,102]$. However, when carefully analyzing the residual uncertainties in even a well described flame, it was determined by Daun et al. [97] that an uncertainty of $48 \%$ is still inherent in the extracted values. It is also clear that 
the soot decay temperature fitting method would result in a thermal accommodation coefficient only valid for sooting flames with a similar local gas composition. Since local gas composition changes rapidly in the counterflow flames utilized in this study, a large thermal accommodation coefficient uncertainty is expected. When the variability in accommodation coefficients found in literature is evaluated along with the inherent uncertainties in LII derived thermal accommodation coefficients, it is estimated that an uncertainty of $50 \%$ is warranted when applying one global thermal accommodation coefficient applicable to the entire counterflow field.

$W_{v}$

The uncertainty in the molar weight of vaporized carbon due to sublimation $\left(W_{v}\right)$ was estimated from a fit to vaporized species calculations by Leider et al. [85]. The fit varies from 26 to 42 grams/mole between $2000 \mathrm{~K}$ and $4200 \mathrm{~K}$. Lacking any information on uncertainty from the original work, the standard uncertainty in the molar weight of vaporized carbon is estimated as 4 grams/mole largely based on the temperature variability. The use of low fluence LII in this work where sublimation of carbon from the surface of soot is small to negligible lends credence that the sublimation terms and their estimated uncertainty play a minimal role in the uncertainty of derived LII variables.

$\alpha_{M}$

The vaporization mass accommodation coefficient represents the fraction of carbon molecules escaping the heated soot particle surface to the molecules reabsorbed into the particle after surface collision. Individual accommodation coefficients are used by Michelsen $[70,83]$ to account for the vaporization of $\mathrm{C} 1$ and larger carbon clusters. However, in the regime of low fluence LII where vaporization plays a minimal role, many researchers utilize an average mass accommodation coefficient to account for the average mass of sublimated carbon clusters [70]. The mass accommodation 
coefficient remains relatively unknown, and as such, values for the mass accommodation coefficient vary in literature from 0.5 to 1 . Considering a uniform distribution with a width of 0.5 , the standard uncertainty in the vaporization mass accommodation coefficient used in this work is taken as 0.15 .

$p_{\text {ref }}$

The uncertainty in the reference pressure $\left(p_{r e f}\right)$ used in the Clausius-Clapeyron equation to calculate the equilibrium partial pressure of sublimed carbon is negligible since the reference pressure of 1 atmosphere was the total vapor pressure setpoint for calculation of the reference temperature.

$\Delta H_{v}$

The uncertainty in the enthalpy of formation $\left(\Delta H_{v}\right)$ for sublimated carbon clusters was estimated from two inferences. Data from Leider et al. [85] demonstrates evidence that C3 is the primary sublimated species at laser induced incandescence temperatures. NIST JANAF data [103] reported C3 carbon enthalpy of formation uncertainty as $\pm 13 \mathrm{KJ} / \mathrm{mol}$ at $2200 \mathrm{~K}$. It is beneficial to develop an average enthalpy of formation for all sublimed carbon clusters at high temperature. A comparison of data reveals that values reported by Melton [53], averages from Leider et al., and averaged NIST JANAF data for C1, C2, and C3 carbon clusters all agree to within $\pm 15 \mathrm{KJ} / \mathrm{mol}$. This analysis yielded a reasonable standard uncertainty estimate of $\pm 15 \mathrm{KJ} / \mathrm{mol}$ on the enthalpy of formation used in this work due to the agreement between documented uncertainty and independently published data.

$T_{\text {ref }}$

The uncertainty in the temperature at which the total pressure of sublimated carbon species reaches one atmospheric pressure, or the reference temperature $\left(T_{r e f}\right)$ was estimated from data by Lieder et al. [85] and NIST JANAF data [103] for C3 carbon. The nominal value published by Leider et al. is given as $3915 \mathrm{~K}$ from calculations 
using other measured thermal properties of carbon and in good agreement with carbon arc temperature measurements. NIST JANAF data provides a value of $4136 \mathrm{~K}$ for $\mathrm{C} 3$ carbon. The difference in the two reported values provides the width of the uniform distribution as $200 \mathrm{~K}$. The standard uncertainty estimate thus is set at $60 \mathrm{~K}$.

$E(m)$

The absorption function $E(m)$ is a function of the complex index of refraction of soot. Accurately determining a value for this variable has proven challenging to the scientific community. Hence the uncertainty on the absorption function is large due to several factors relevant to high temperature from LII. A thorough review on the index of refraction of soot by Bond and Bergstrom [72] report the absorption function ranging from 0.15 to 0.24 . The complex index of refraction data used to determine the absorption function was collected with a range of measurement techniques, flame configurations, and carbonaceous fuels at temperatures from room air ambient to flame temperature. It is well known however, that many substances undergo complex index of refraction changes with a change in temperature. While in general, temperature tends to affect infrared wavelength absorption more strongly than visible absorption, at LII temperatures of $3600 \mathrm{~K}, k_{B} T$ thermal energy begins to approach near infrared and visible photon energies and may start to affect near infrared and visible laser light absorption [90]. As such, research has been conducted utilizing high temperature LII and a well described flame to determine a more relevant value for the absorption function at high temperature. Snelling et al. [101] reported a value of around 0.4 . It is also worth noting that high temperature experiments may also give rise to phase change, and a corresponding change in the optical properties of the substance. Work by Michelsen [83] mentioned this possibility and the data suggests that the best fit between modeled and experimental LII data may lie somewhere between the absorption function of solid and liquid soot if some melting is achieved. While the phase diagram for graphite does not exhibit a liquid phase below 100 atm and 
$4000 \mathrm{~K}$, other soot morphologies may melt at LII conditions. Further investigation into these mechanisms would be beneficial to the scientific community. Taking all of these uncertainties into consideration, a standard uncertainty bound was estimated for the absorption function with a lower bound of 0.2 and upper bound of 0.38 .

$\rho_{s}$

Uncertainty in the density of soot $\left(\rho_{s}\right)$ is estimated through several considerations. Thermal expansion calculations compared to experimental data reported by Fried and Howard [84] agree within approximately $1 \%$ or less for temperatures up to $3500 \mathrm{~K}$. Upon consideration of the uncertainty of the exact morphology of soot formed by different fuel and flame configurations, measurements of soot density exhibit a spread of about $10 \%$ in literature [70]. A $10 \%$ uncertainty was also reached by Daun et al. [98] and will be adopted in this work as the standard uncertainty.

$c_{s}$

Uncertainty in the heat capacity of soot $\left(c_{s}\right)$ is analyzed from documented uncertainty and variation between published measurements. The temperature dependent formulation adopted in this work was originally published by Fried and Howard [84] and agrees well with JANAF data [103]. Uncertainties of 3-5\% at temperatures up to $3500 \mathrm{~K}$ were documented in the JANAF publication. Overall agreement between different experimental measurements reported in literature $[70,103]$ due to changes in soot morphology increases the standard uncertainty estimate to $10 \%$ in this work.

\section{$Q_{\text {conduction }}$}

The uncertainty of the conduction model ( $\left.Q_{\text {conduction }}\right)$ was estimated by examining the difference between the highest volumetric heat loss result (Sherman model [73] with integrated mean heat transfer properties) and the lowest (McCoy and Cha [86]) for the transition regime. While the difference between the two models is affected strongly by pressure and local gas temperature, an average deviation between the 
two models over the experimental operating conditions used in this work provided an estimate of approximately $12 \%$ for the conduction model standard uncertainty.

$\operatorname{pdf}\left(d_{p}\right), \operatorname{pdf}\left(N_{p}\right)$

This analysis does not include uncertainties due to particle diameter distribution or particle aggregation. Instead these are freely variable input assumptions based on other measurement techniques. Transmission electron microscopy (TEM), and elastic light scattering measurements are used in this work to provide insight into these input parameters. Aggregate soot particles do have a large effect on conduction heat transfer $(\sim 40 \%)$ and primary particle sizing so accurate knowledge of this input parameter is important. Primary particle size distribution plays a smaller role. Neither input has a significant effect on volume fraction measurements. Detailed work on the uncertainty of these quantities can be found in literature [76-78, 104].

A summary of identified variables and underlying uncertainties are listed in Table 3.1. The demarcation in the table identifies the experimental variables (top) and model variables (bottom).

\subsubsection{Uncertainty Analysis of LII Measurements}

An attempt was made to utilize proper statistical uncertainty methods where possible. All uncertainties documented here are standard uncertainty estimates. Expansion of uncertainty to $95 \%$ confidence is not particularly meaningful due to a lack of statistics and incomplete uncertainties on parameters found in literature. In the LII model, all variables were perturbed by their respective uncertainties. The analysis does not include second order effects. The resulting uncertainties corresponding to extracted variables of soot volume fraction, primary particle size, and soot number density are listed in Table 3.2 by descending order of importance. The total uncertainty listed at the bottom of the table is the square 
Table 3.1: List of parameters involved in Laser Induced Incandescent analysis and their associated uncertainties. Experimental and model variables/parameters are listed at the top and the bottom, respectively.

\begin{tabular}{lllll}
\hline \hline Param. & Uncert. & $\%$ & Units & Description \\
\hline$I_{L I I}$ & - & 7 & Counts & Soot Incandescence \\
$\eta$ & - & 6 & $\mathrm{~W} / \mathrm{nmCount}$ & Detector Calibration Factor \\
$\Omega$ & $7 \mathrm{E}-4$ & 2 & $\mathrm{Sr}$ & Detector Solid Angle \\
$A_{e}$ & $4.69 \mathrm{E}-8$ & 2 & $\mathrm{~cm}^{2}$ & Calibrated Experimental Pixel Area \\
$y$ & - & - & $\mathrm{cm}$ & Laser Sheet Spatial Width \\
$F_{0}$ & - & 8 & $\mathrm{~J} / \mathrm{cm}^{2}$ & Laser Fluence \\
$p_{0}$ & $3.5 \mathrm{E}-2$ & - & $\mathrm{atm}$ & Ambient Pressure \\
\hline$T_{0}$ & 250 & - & $\mathrm{K}$ & Local Gas Temperature \\
$\gamma$ & $1.4 \mathrm{E}-2$ & - & unitless & Local Gas Heat Capacity Ratio \\
$\kappa$ & $6 \mathrm{E}-4$ & - & $\mathrm{W} / \mathrm{cmK}$ & Local Gas Thermal Conductivity \\
$W_{g}$ & 0.5 & - & $\mathrm{g} / \mathrm{mol}$ & Local Gas Average Molar Weight \\
$\alpha_{T}$ & - & 50 & unitless & Heat Conduction Accommodation Coefficient \\
$W_{v}$ & 4 & - & $\mathrm{g} / \mathrm{mol}$ & Molar Weight of Vaporized Carbon \\
$\alpha_{M}$ & 0.15 & - & unitless & Vaporization Mass Accommodation Coefficient \\
$p_{r e f}$ & - & - & atm & Reference Pressure for Clausius-Clapeyron Eq. \\
$\Delta H_{v}$ & 15 & - & $\mathrm{kJ} / \mathrm{mol}$ & Enthalpy of Formation of Sublimed Carbon \\
$T_{r e f}$ & 60 & 1.5 & $\mathrm{~K}$ & Reference Temperature for Clausius-Clapeyron Eq. \\
$E(m)$ & $0.2-0.38$ & - & unitless & Absorption Function \\
$\rho_{s}$ & - & 10 & $\mathrm{~g} / \mathrm{cm}{ }^{3}$ & Soot Density \\
$c_{s}$ & - & 10 & $\mathrm{~J} / \mathrm{gK}$ & Soot Heat Capacity \\
$Q_{c o n d}$ & - & 12 & $\mathrm{~W}$ & Conduction Model \\
& & & &
\end{tabular}

root of the sum-squared error of all uncertainties (RSS). The generic equation is given as

$$
\Delta f_{v}, d_{p}=\sqrt{\sum_{i=1}^{N} \xi_{i}^{2}}
$$

Where $\xi$ is a generic LII model variable and the sum is over all $N$ variables. A simpler practical example of the origin of the RSS error method is given in Chapter 3.2. It is clear that the absorption function, which directly affects the measurement of incandescent signal and hence volume fraction, introduces the largest uncertainty on the volume fraction measurement. Also as expected, uncertainty in the primary particle size is dominated by uncertainties affecting the conduction heat transfer model. Sublimation terms are found to be negligible for all three extracted variables due to the use of low-fluence LII in this 
work. Since the number density is extracted from measurements of soot volume fraction and particle size, the uncertainty of the number density due to any variable and the total uncertainty is given by,

$$
\Delta N=\sqrt{3 \Delta d_{p}^{2}+\Delta f_{v}^{2}}
$$

yielding a much larger uncertainty compared to soot volume fraction and particle size measurements.

Table 3.2: Uncertainties in Laser Induced Incandescence extracted variables.

\begin{tabular}{llllll}
\hline \hline \multicolumn{2}{c}{ Volume Fraction } & \multicolumn{2}{c}{ Primary Particle Size } & \multicolumn{2}{c}{ Number Density } \\
\hline Parameter & Uncertainty & Parameter & Uncertainty & Parameter & Uncertainty \\
\hline$E(m)$ & $30 \%$ & $\alpha_{T}$ & $42 \%$ & $\alpha_{T}$ & $72 \%$ \\
$T_{0}$ & $17 \%$ & $T_{0}$ & $30 \%$ & $T_{0}$ & $55 \%$ \\
$I_{L I I}$ & $7 \%$ & $\gamma$ & $18 \%$ & $\gamma$ & $30 \%$ \\
$\eta$ & $6 \%$ & $\rho_{s}$ & $10 \%$ & $E(m)$ & $30 \%$ \\
$\gamma$ & $5 \%$ & $c_{s}$ & $10 \%$ & $\rho_{s}$ & $20 \%$ \\
$\rho_{s}$ & $5 \%$ & $Q_{\text {cond }}$ & $8 \%$ & $c_{s}$ & $20 \%$ \\
$c_{s}$ & $5 \%$ & $\kappa$ & $4 \%$ & $Q_{\text {cond }}$ & $14 \%$ \\
$\alpha_{T}$ & $4 \%$ & $F_{0}$ & $4 \%$ & $F_{0}$ & $8 \%$ \\
$W_{g}$ & $4 \%$ & $W_{g}$ & $4 \%$ & $W_{g}$ & $8 \%$ \\
$F_{0}$ & $4 \%$ & $p_{0}$ & $2 \%$ & $\kappa$ & $7 \%$ \\
$Q_{\text {cond }}$ & $2 \%$ & $\Delta H_{v}$ & 0 & $I_{L I I}$ & $7 \%$ \\
$\Omega$ & $2 \%$ & $T_{r e f}$ & 0 & $\eta$ & $6 \%$ \\
$A_{e}$ & $2 \%$ & $I_{L I I}$ & 0 & $p_{0}$ & $4 \%$ \\
$p_{0}$ & $2 \%$ & $\eta$ & 0 & $\Omega$ & $2 \%$ \\
$\kappa$ & 0 & $\Omega$ & 0 & $A_{e}$ & $2 \%$ \\
$\Delta H_{v}$ & 0 & $A_{e}$ & 0 & $\Delta H_{v}$ & 0 \\
$T_{r e f}$ & 0 & $y$ & 0 & $T_{r e f}$ & 0 \\
$y$ & 0 & $W_{v}$ & 0 & $y$ & 0 \\
$W_{v}$ & 0 & $\alpha_{M}$ & 0 & $W_{v}$ & 0 \\
$\alpha_{M}$ & 0 & $p_{r e f}$ & 0 & $\alpha_{M}$ & 0 \\
$p_{r e f}$ & 0 & $E(m)$ & 0 & $p_{r e f}$ & 0 \\
\hline Total & $38 \%$ & Total & $57 \%$ & Total & $106 \%$ \\
& & & & &
\end{tabular}

\subsubsection{Mixture Averaged Thermal Accommodation Coefficient, $\alpha_{T}$}

The reported uncertainties in Table 3.2 are conservative estimates of which there is clear room for improvement. By targeting some of the most important uncertainties impacting LII extracted variables we can hope to significantly improve the uncertainty in the LII tech- 
nique overall. Clearly the thermal accommodation coefficient between soot and the local gas species $i\left(\alpha_{T, i}\right)$ is the largest identified uncertainty on the primary particle size listed in Table 3.2. A thorough review of thermal accommodation literature provided enough data to explore a species mixture averaged $\alpha_{T}=\sum \alpha_{T, i} X_{i}$ at each spatial location in the flame. The concept is consistent with the current strategy of spatially varying mixture averaged $\kappa$ and $\gamma$ by solving gas-phase conservation equations. This strategy is motivated by a hypothesis that the uncertainty of $\alpha_{T}$ can be reduced. The strategy considered molecular dynamics (MD) determined values and experimental measurements compiled from literature. From quasi one-dimensional simulations as described in Chapter 2.2, the top 5 most important species were determined at each spatial location on a molar basis and they typically represent approximately $95 \%$ of the total molar concentration. By considering the top 5 species at all spatial locations and for all test cases, a final list of ten most important species were included in the subsequent uncertainty analysis. Figure 3.1 shows the computed species concentration profiles along the axis of symmetry for two test cases of non-premixed ethylene-oxygen counterflow flames (a) at 4.065 atmospheres with opposed nitrogen diluted fuel and air streams $\left(X_{C_{2} H_{4},-L / 2}=0.5\right.$ and $\left.X_{O_{2},+L / 2}=0.2101\right)$ at a strain rate of $119 \mathrm{~s}^{-1}$, and (b) at 14 atmospheres with opposed fuel and oxygen streams diluted with helium $\left(X_{C_{2} H_{4},-L / 2}=0.41\right.$ and $\left.X_{O_{2}, L / 2}=0.1723\right)$ at a strain rate of $135 \mathrm{~s}^{-1}$. The local gas temperature profiles are also shown. In Fig. 3.1 the fuel flow originates from the left boundary $(z=-L / 2)$ and the oxidizer from the right boundary $(z=+L / 2)$ where $L$ is the nozzle separation, setting up a fuel rich region in the negative $z$-direction, and an oxidizer rich region in the positive $z$-direction with $z=0$ identifying the center plane between the nozzles. The red dotted vertical lines show the region of interest corresponding to the soot layer location in the flow field. Narrowing the analysis to this region further reduces the list of most important species to eight, which are identified as $\mathrm{N}_{2}, \mathrm{He}, \mathrm{C}_{2} \mathrm{H}_{4}, \mathrm{H}_{2}, \mathrm{H}_{2} \mathrm{O}$, $\mathrm{CO}, \mathrm{CO}_{2}$, and $\mathrm{C}_{2} \mathrm{H}_{2} . \mathrm{O}_{2}$ is insignificant at the spatial region where soot is formed under all tested counterflow conditions. 


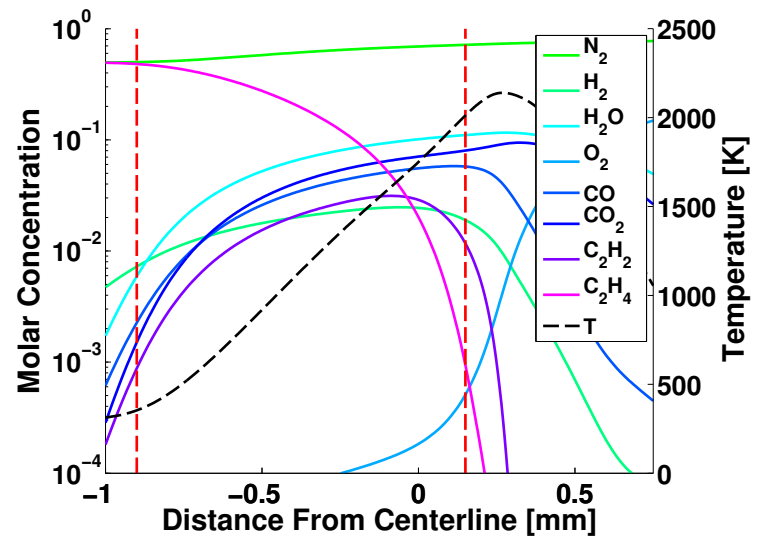

(a) Nitrogen diluted $X_{C 2 H 4,-L / 2}=0.5$ and $X_{O 2,+L / 2}=0.2101$ non-premixed counterflow flame structure at a pressure of $4.065 \mathrm{~atm}$ and strain rate of $119 \mathrm{~s}^{-1}$.

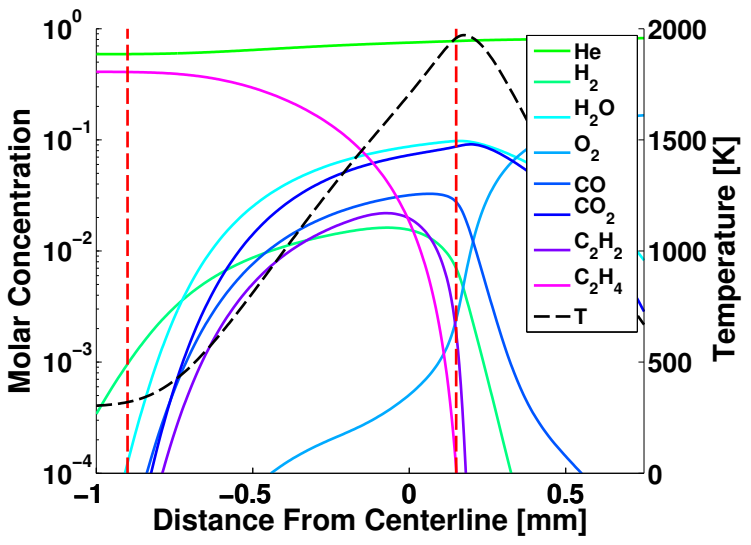

(b) Helium diluted $X_{C 2 H 4,-L / 2}=0.41$ and $X_{O 2,+L / 2}=0.1723$ non-premixed counterflow flame structure at a pressure of $14 \mathrm{~atm}$ and strain rate of $135 \mathrm{~s}^{-1}$

Figure 3.1: Profiles of temperature and major species mole fractions with identification of soot layers given by red vertical lines.

It is well accepted [105-107] that $\alpha_{T, i}$ increases with increasing gas molecular mass and decreases with gas molecule complexity. The former is at least partially influenced by increased gas-surface interaction time (lower speed and deeper/wider potential well for larger molecules) resulting in a higher probability that the gas molecule will equilibrate to the surface temperature. The latter is an effect of difficulty in accommodating internal energy modes. Thermal accommodation is additionally a function of gas and surface temperature. For monatomic gases, thermal accommodation generally increases with increasing gas temperature and remains constant or decreases slightly with increasing surface temperature due to complex functions of molecular mass and gas-surface interaction. The effects have been exhibited in both experiments and simulations with the exception of argon $[105,106]$. For polyatomic gases, evidence suggests that thermal accommodation decreases at both higher gas and surface temperatures dominated by ineffective accommodation to internal energy modes, especially vibrational modes. Low gas and surface temperatures increase interaction time between gas molecule and surface resulting in greater energy exchange to internal energy modes and higher thermal accommodation. The converse is true for higher temperature yet shows evidence of asymptotic behavior as temperature is increased 
to about $1000 \mathrm{~K}[98,106,108]$. The asymptotic behavior offers evidence that experimental data collected at gas and surface temperatures around $1000 \mathrm{~K}$ can be relevant for LII studies at much higher temperatures. It is thus assumed here that thermal accommodation is constant for the temperature range of approximately $1000 \mathrm{~K}$ and above. Literature sources with $\alpha_{T, i}$ values reported in the low temperature regime were not considered in the analysis. All $\alpha_{T, i}$ data reported were for species collision on a graphite or soot surface. It is assumed in this case that graphite and soot are acceptable analogs, as soot is believed to anneal around $2500 \mathrm{~K}$ forming a graphitic surface structure. All species dependent thermal accommodation coefficients reported in literature most relevant to LII (high gas and surface temperature) for the eight most important species supplemented with $\mathrm{O}_{2}$ and $\mathrm{CH}_{4}$ species common to other flame configurations are analyzed in detail. No data could be found for $\alpha_{T, i}$ of the $\mathrm{OH}$-graphite pair. Clearly $\mathrm{CO}_{2}, \mathrm{H}_{2} \mathrm{O}, \mathrm{OH}$, and $\mathrm{O}_{2}$ thermal accommodation measurements at high surface and gas temperatures are complicated by surface reactions. The lack of a sufficient population of data for any species makes the calculation of uncertainty difficult and inaccurate on statistical bounds. Instead the uncertainties were estimated conservatively based on the scatter in the data assuming a uniform distribution with consideration to the reported error in literature. The maximum error reported in literature was used as a conservative estimate of measurement error. The large uncertainty of LII derived thermal accommodation from Daun et al. [97,98] for individual species $(\sim 50 \%)$ was acknowledged but not directly considered in the uncertainty analysis when less uncertain results were available relevant to LII and high temperature conditions. Details for each species analyzed are as follows.

\section{$\mathrm{CO}_{2}$}

Three studies $[97,98,107]$ reported data for $\mathrm{CO}_{2}$ all in good agreement within $10 \%$. The standard deviation of the data assuming a uniform distribution is $3 \%$. The average was taken as the nominal value. The root sum squared error estimate for $\mathrm{CO}_{2}$ was calculated as $15 \%$ using the standard deviation of the data and the largest error 
reported in literature.

CO

Three studies $[97,98,109]$ reported values for CO. Simulations and experiments by Daun et al. [98] are in good agreement with data from Watt [109] reported somewhat higher. An analysis of the experimental method used by Watt shows data collected at high surface temperature with room temperature gas, resulting in accommodation coefficient measurements higher than that for high gas and surface temperature LII conditions. The nominal thermal accommodation value for $\mathrm{CO}$ was set at 0.3 . The standard deviation of $15 \%$ was calculated from the spread of the data assuming a uniform distribution. A total root sum squared error of $16 \%$ was calculated including the maximum reported measurement error of $5 \%$.

$\mathbf{N}_{2}$

Four studies $[97,98,100,107]$ reported thermal accommodation data for nitrogen, three of which were found in good agreement. Results by Kamat et al. [107] were significantly lower, possibly due to an in-house optimization of N-C molecular interaction parameters unique to their approach. Results for monatomic gases reported by Kamat et al. were in good agreement with other simulations. The nominal thermal accommodation for $\mathrm{N}_{2}$ was set at 0.26 , the median of the samples. Total root sum squared error was calculated as $20 \%$ based on the maximum reported error applied to the median (11\%) and standard deviation of the spread of the data $(17 \%)$.

\section{He}

All thermal accommodation values for helium reported in literature $[97,98,106,107]$ were in good agreement. The nominal value for helium was set at the average, and the root sum square standard uncertainty was calculated as $15 \%$ using the largest uncertainty specified in literature applied to the mean (15\%) and the standard deviation of the samples $(4 \%)$. 
$\mathrm{CH}_{4}$

The spread in the data for $\mathrm{CH}_{4}$ was more significant between the four sources in literature $[97,98,106,107]$ relevant to high temperature conditions. The average of the data was taken as the nominal value. The standard deviation from the spread in the data $(18 \%)$ and the maximum error reported in literature applied to the mean $(16 \%)$ resulted in a total standard error estimate of $24 \%$.

$\mathbf{H}_{2} \mathbf{O}$

Watt [109] reported the only thermal accommodation data for the $\mathrm{H}_{2} \mathrm{O}$-graphite pair for high surface temperature and a room temperature gas at low pressure. As a result, the reported value may be somewhat high. A conservative $50 \%$ error estimate was assumed, which covers the plausible thermal accommodation range of other simple nonlinear polyatomic molecules.

$\mathbf{H}_{2}$

Data reported for $\mathrm{H}_{2}[100,106,108-110]$ were in relatively good agreement. The mean was chosen as the nominal value. The standard deviation from the spread in the data $(10 \%)$ and maximum error reported in literature applied to the mean (14\%) resulted in a total standard error of $17 \%$.

$\mathbf{O}_{2}$

No thermal accommodation data for the $\mathrm{O}_{2}$-graphite pair were found in literature. Given that $\mathrm{O}_{2}$ is a linear polyatomic molecule, it should be expected to fall within a range of thermal accommodation values for other linear polyatomic molecules. Thermal accommodation by Rosenblatt et al. [111] for $\mathrm{N}_{2}$ and $\mathrm{O}_{2}$ on nickel, copper, and platinum surfaces were very similar albeit using a low temperature experimental approach. Taking this into account, thermal accommodation for $\mathrm{O}_{2}$ was set equal to that of $\mathrm{N}_{2}$ with an uncertainty set at $50 \%$, which covers the range of thermal accommodation values for other linear polyatomic molecules. 
$\mathbf{C}_{2} \mathbf{H}_{4}$

Only Daun et al. [97] reported thermal accommodation for $\mathrm{C}_{2} \mathrm{H}_{4}$ using a highly uncertain LII technique to determine the value. It is worth noting that the results for $\mathrm{C}_{2} \mathrm{H}_{4}$ were corrected from Daun et al. 2008 [97] to the values consistent in Daun et al. 2009 [98] due to a change in the heat transfer model used between the two published works. Values for other similar nonlinear polyatomic molecules $\left(\mathrm{CH}_{4}, \mathrm{C}_{2} \mathrm{H}_{6}\right)$ reported here and in literature provided additional insight. The nominal value of thermal accommodation for $\mathrm{C}_{2} \mathrm{H}_{4}$ was set at 0.1 with an uncertainty of $50 \%$ consistent with the uncertainty of the LII derived results. The uncertainty covers the range of values reported for other similar nonlinear polyatomic molecules.

\section{$\mathbf{C}_{2} \mathbf{H}_{2}$}

No sources were found for thermal accommodation of the $\mathrm{C}_{2} \mathrm{H}_{2}$-graphite pair. Low temperature thermal accommodation by Rosenblatt et al. [111] for $\mathrm{C}_{2} \mathrm{H}_{2}$ and $\mathrm{CO}_{2}$ on a copper surface was reported as similar. Considering both are linear polyatomic molecules of roughly equal complexity, the thermal accommodation coefficient of $\mathrm{C}_{2} \mathrm{H}_{2}$ was set equal to that for $\mathrm{CO}_{2}$ with an uncertainty of $50 \%$, covering a wide range of thermal accommodation values for other linear polyatomic molecules.

A summary of the results is given in Table 3.3.

Table 3.3: Relevant species thermal accommodation coefficients $\left(\alpha_{T, i}\right)$ and documented uncertainties from literature.

\begin{tabular}{|c|c|c|c|c|c|c|c|c|c|}
\hline \multicolumn{4}{|c|}{ Simulations } & LII Exp. & \multicolumn{5}{|c|}{ Experiments } \\
\hline Species & [107] & [98] & [108] & [97] [98] & [109] & [110] & [100] & [106] & [111] \\
\hline $\mathrm{CO}_{2}$ & $0.2 \pm 15 \%$ & $0.2 \pm 5 \%$ & & $0.18 \pm 50 \%$ & & & & & \\
\hline $\mathrm{CO}$ & & $0.3 \pm 5 \%$ & & $0.27 \pm 50 \%$ & 0.46 & & & & \\
\hline $\mathrm{N}_{2}$ & $0.12 \pm 25 \%$ & $0.27 \pm 5 \%$ & & $0.26 \pm 50 \%$ & & & $0.26 \pm 8 \%$ & & \\
\hline $\mathrm{He}$ & $0.22 \pm 14 \%$ & $0.22 \pm 5 \%$ & & $0.21 \pm 50 \%$ & & & & $0.19 \pm 10 \%$ & \\
\hline $\mathrm{CH}_{4}$ & $0.16 \pm 13 \%$ & $0.11 \pm 5 \%$ & & $0.09 \pm 50 \%$ & & & & $0.17 \pm 10 \%$ & \\
\hline $\mathrm{H}_{2} \mathrm{O}$ & & & & & 0.22 & & & & \\
\hline $\mathrm{H}_{2}$ & & & 0.12 & & 0.13 & 0.15 & $0.17 \pm 12 \%$ & $0.15 \pm 10 \%$ & \\
\hline $\mathrm{O}_{2}$ & & & & & & & & & $\sim 0.3$ \\
\hline $\mathrm{C}_{2} \mathrm{H}_{4}$ & & & & $0.1 \pm 50 \%$ & & & & & \\
\hline $\mathrm{C}_{2} \mathrm{H}_{2}$ & & & & & & & & & $\sim 0.19$ \\
\hline
\end{tabular}


The final values for single species-graphite pair thermal accommodation and their standard uncertainties are given in Table 3.4. The review of literature clearly shows additional work is necessary to provide a more thorough and less uncertain set of data for species dependent thermal accommodation coefficients. Species mixture averaging of the thermal accommodation data collected for the top five most important species shows that thermal accommodation varies in the sooting region between 0.18 and 0.26 for the nitrogen diluted flame at $4.065 \mathrm{~atm}$ described above and 0.16 to 0.22 for the helium diluted flame at $14 \mathrm{~atm}$. Both ranges are significantly lower than the nominal value reported in the LII literature [70], i.e. $0.28-0.38$. Considering the importance of the thermal accommodation coefficient, accurate fits for species-surface pair $\alpha_{T, i}$ as a function of gas and surface temperature with clearly defined uncertainty should be a major focus of the scientific community.

Table 3.4: List of top 10 most important species thermal accommodation coefficients $\left(\alpha_{T, i}\right)$ and uncertainty.

\begin{tabular}{lll}
\hline \hline Species & $\alpha_{T, i}$ & Uncert. \\
\hline $\mathrm{CO}_{2}$ & 0.193 & $15 \%$ \\
$\mathrm{CO}$ & 0.3 & $16 \%$ \\
$\mathrm{~N}_{2}$ & 0.26 & $20 \%$ \\
$\mathrm{He}$ & 0.21 & $15 \%$ \\
$\mathrm{CH}_{4}$ & 0.13 & $24 \%$ \\
$\mathrm{H}_{2} \mathrm{O}$ & 0.22 & $50 \%$ \\
$\mathrm{H}_{2}$ & 0.144 & $17 \%$ \\
$\mathrm{O}_{2}$ & 0.3 & $50 \%$ \\
$\mathrm{C}_{2} \mathrm{H}_{4}$ & 0.1 & $50 \%$ \\
$\mathrm{C}_{2} \mathrm{H}_{2}$ & 0.193 & $50 \%$
\end{tabular}

The uncertainty of the species mole fraction weighted thermal accommodation coefficient on soot volume fraction, particle size, and number density was analyzed by comparing the nominal mixture thermal accommodation results to the those perturbed by uncertainties of $\alpha_{T, i}$ listed in Table 3.4. Since all the species exhibit different estimated uncertainties and the order of species importance changes spatially, clearly the mixture uncertainty also changes spatially. The effect of spatial uncertainty on the total uncertainty for thermal accommodation was small $(<10 \%)$ compared to the mixture averaged uncertainties 
reported in Table 3.4, resulting in only a $2 \%$ increase in total error on primary particle size in the most extreme case. For completeness, this error is included in the total uncertainties reported here. For nitrogen-diluted flames, the maximum standard uncertainties on volume fraction, particle size, and number density were 5\%,32\%, and 56\% respectively. For helium-diluted flames, the maximum standard uncertainties on volume fraction, particle size, and number density were $4 \%, 22 \%$, and $38 \%$ respectively. The effect on the total uncertainty of volume fraction remained small and unchanged for both helium and nitrogen-diluted flames. The effect of decreased $\alpha_{T}$ uncertainty lowers the total uncertainty on particle size and number density for nitrogen-diluted flames to $50 \%$ and $92 \%$, respectively, while that of helium-diluted flames are decreased to $44 \%$ and $83 \%$, respectively.

\subsubsection{Estimation of Spatially Varying Absorption Function}

The absorption function is the most important variable for controlling the uncertainty on volume fraction, as shown in Table 3.2. The optical properties of soot are dependent on the three physical processes associated with free carriers, lattice vibrations, and electronic transitions, as well as impurities from molecular composition (e.g. H/C ratio) [112]. All these dominant processes are affected by changes in bond structure $\left(\mathrm{sp}^{2}, \mathrm{sp}^{3}, \pi\right.$, and free electrons) and temperature. In general lattice vibration effects on the polarizability of a material only apply to infrared and longer wavelengths. LII is primarily concerned with visible wavelengths, so the lattice vibration effects are neglected here. Uncertainty analysis for the visible spectrum using Drude-Lorentz dispersion theory from Lee and Tien [113] for graphite indicates the complex index of refraction changes significantly with large temperature gradients. An increase in temperature of greater than $1000 \mathrm{~K}$ increases absorption by approximately $40 \%[114,115]$. Thermal expansion also plays a role in affecting optical properties [112] but its affect is small compared to the temperature dependence of the polarizability of soot (see Michelsen et al. [116] for a nice review on soot thermal expansion). Variation in soot composition reflected by a change in electron number density by a factor 
of two also changes absorption by $30 \%$ or greater $[114,115]$.

It is known in literature that soot contains $\mathrm{sp}^{3}$ and $\mathrm{sp}^{2}$ hybridized bonds as well as impurities of $\mathrm{H}_{2}, \mathrm{O}_{2}$, and possibly other compounds depending on local gas composition in the flame [72]. From band-gap theory, it is suggested that the clustering of $\mathrm{sp}^{2}$ bonds determines the optical properties and the absorption characteristic of soot. An increase in $\mathrm{sp}^{2}$ bonds results in a decrease in the optical gap and an increase in absorption. This can be controlled by adding or removing specific species from the local environment (e.g. increased hydrogen decreases $\mathrm{sp}^{2}$ bonding [72]) or by raising or lowering the particle temperature to induce or inhibit graphitization. For the case of flame studies using LII, both an increase in flame temperature and laser heating of soot would result in increased annealing and graphitization. Similarly, increased residence time at higher temperature increases graphitization and optical absorption (See Michelsen [83] for a thorough review of annealing applied to LII). Particle growth has also been postulated to affect soot optical properties with evidence supported by theory and experiment. The addition of layer structure as particles grow in size has been observed to increase amorphous carbon and soot opacity [72,117].

The nonpremixed counterflow configuration employed in this study adds additional complexity with the competing effects of soot precursor concentration and temperature variation along the particle trajectory which is unique from other burner configurations. Several researchers ( [72] and citations within) have also suggested that aggregation of particles may increase absorption efficiencies by as much as $50 \%$ depending on the number and size of primary particles. The counterflow flame configuration and the controllable residence time of the flow field inhibits strong aggregation unlike other burner configurations, but some aggregation still may play a role in determining optical absorption. Uncertainties due to a phase change of soot are assumed negligible due to the use of low fluence LII which inhibits significant sublimation of carbon from the solid soot particle surface. A change in optical properties due to melting is also considered unlikely. The phase diagram for graphite indicates melting is not achievable under a pressure of $100 \mathrm{~atm}$ and tempera- 
ture under $4200 \mathrm{~K}$ yet may be possible if soot morphology is significantly different from graphite. The above combined effects on the optical properties and absorption of soot are highly speculative and are not well understood. It is clearly possible that the absorption function could suffer from uncertainties as high as $50 \%$ or greater from a nominal assumed value.

The absolute irradiance calibrated LII technique used in this study measures three quantities, (i) temperature from the ratio of two wavelength signal detection, (ii) the absolute magnitude of the detected signals, and (iii) the signal decay in time. Currently volume fraction is determined from signal magnitude, and particle size from the signal decay. It is proposed here that the measurement of temperature be used to quantitatively determine soot optical absorption by targeting the soot absorption function $E(m)$ using a two parameter fitting routine to simultaneously solve for the absorption function and primary particle size. A similar approach was taken by Eremin et. al [118] when investigating the effect of nanoparticle growth on the absorption function using a particle synthesis device. For moderate to heavily sooting flames a clear gradient in LII intensity is noticeable across the flame sheet from extinction of laser energy and LII signal as it passes through the soot layer. In these cases an extinction correction is used as an additional function of $E(m)$ and is iteratively matched with LII results until a converged solution is reached between the two techniques. The extinction correction procedure is outlined in Chapter 2.5 with an assumption of constant volume fraction along the laser beam and signal line of sight paths. An initial guess for the volume fraction is made using LII, where the incandescent signal and laser fluence are corrected until the volume fraction and absorption function reach a converged solution. With the local temperature along the axis of symmetry known from computations and the laser beam fluence profile accurately calibrated and corrected for extinction if present, the soot absorption function can be quantified spatially within an error bound determined from uncertainty analysis of all uncertain variables in the LII technique. This effectively determines the spatially varying absorption function due to various 
large and uncertain physical effects as described previously. The absorption function is assumed constant here between the detection wavelengths $450 \mathrm{~nm}$ and $650 \mathrm{~nm}$ and the laser absorption wavelength of $532 \mathrm{~nm}$. Dispersion theory and optical measurements provide evidence of little change in the complex index of refraction in the visible wavelength range considered here.

An example of a least squares fit for the local absorption function $E(m)$ to account for rapidly changing conditions in the counterflow geometry is shown in Fig. 3.2. The results correspond to flame conditions at $1 \mathrm{~atm}$, a local strain rate of $a_{l}=91 \mathrm{~s}^{-1}, X_{C_{2} H_{4}}=1.0$ with an opposed flow of air $\left(X_{O_{2}}=0.21\right)$. The fuel flow originates from the left boundary $(z=-L / 2)$ and the oxidizer from the right boundary $(z=+L / 2)$ where $L$ is the nozzle separation, setting up a fuel rich region in the negative $z$-direction, and an oxidizer rich region in the positive $z$-direction (stoichiometric flame location is at $z=0.45 \mathrm{~mm}$ where the mixture fraction is $Z_{s t}=1.84$ ). It is interesting to note that the fitted absorption function values shown fall within the range of values reported in the literature $[70,72]$, namely 0.14-0.4. Effects of aggregation are negligible as measured primary particle sizes are small $(\sim 10 \mathrm{~nm})$.

A comparison between LII extracted volume fraction results was also conducted to examine the validity of assuming a least squares fit $E(m)$ and a globally prescribed constant value for $E(m)$. A constant value of 0.24 was assumed for $E(m)$ which falls in the range of values reported by Bond and Bergstrom [72] ( 0.14-0.24) for measurements taken around flame temperature $\sim 2000 \mathrm{~K}$ or lower and values used in LII literature [70] $(\sim 0.18-0.4)$ for temperatures of $\sim 2600 \mathrm{~K}$ or higher. The value 0.24 also corresponds to a rough mean value for the locally fit range of $E(m)$ values determined throughout all LII studies conducted in this work. Figure 3.3 shows the soot volume fraction at $1 \mathrm{~atm}$, a local strain rate of $a_{l}=91 \mathrm{~s}^{-1}, X_{C_{2} H_{4}}=1.0$ with an opposed flow of air $\left(X_{O_{2}}=0.21\right)$. The assumption of constant $E(m)=0.24$ is given as the light blue line with the light blue shaded region representing the total standard uncertainty bounds from Table 3.5. The volume fraction 


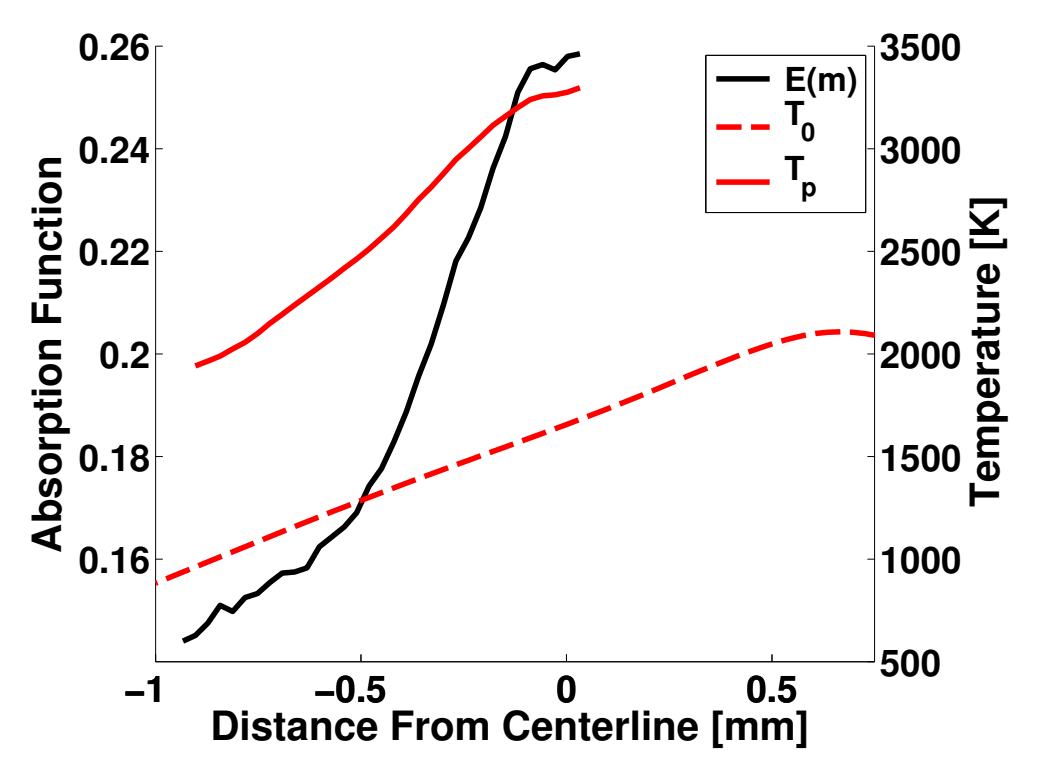

Figure 3.2: LII fitting results for spatially varying absorption function $E(m)$ at $1 \mathrm{~atm}$, $a_{l}=91 \mathrm{~s}^{-1}, X_{C_{2} H_{4}}=1.0$ with opposed flow of air. Also shown are the peak particle temperature (solid red line from experiments) and the local gas temperature (dashed red line from computations).

result from the least squares fit assumption of $E(m)$ is indicated by the black line with the gray shaded region representing the total standard uncertainty bounds from Table 3.5. The red dashed line represents the flow field temperature profile due to the flame where the soot is shown forming on the fuel rich side of the flame. It is evident that the uncertainty in the two results overlap, as they do for all cases examined during this study. Yet the nominal volume fraction profiles show markedly different peaks. This is explained upon consideration that the peak particle temperature near the maximum soot volume fraction is only $\sim 2000 \mathrm{~K}$ on the order of a typical flame temperature due to the local gas temperature being only $\sim 1000 \mathrm{~K}$. In this case, measurements taken at flame temperatures reported by Bond and Bergstrom [72] ( 0.14-0.24) are probably more relevant to the maximum volume fraction region. Furthermore, values for $E(m)$ assumed by the LII community [70] ( $\sim 0.18$ 0.4 ) are more appropriate for the soot inception region near $z=0$ in Fig. 3.3 where the peak particle temperature is high $\sim 3400 \mathrm{~K}$. Not surprisingly, the least squares fit $E(m)$ results shown in Fig. 3.2 support the above explanation. Thus, for the case of the counterflow flame geometry, the rapidly changing local conditions warrant a local fitting of $E(m)$ to 


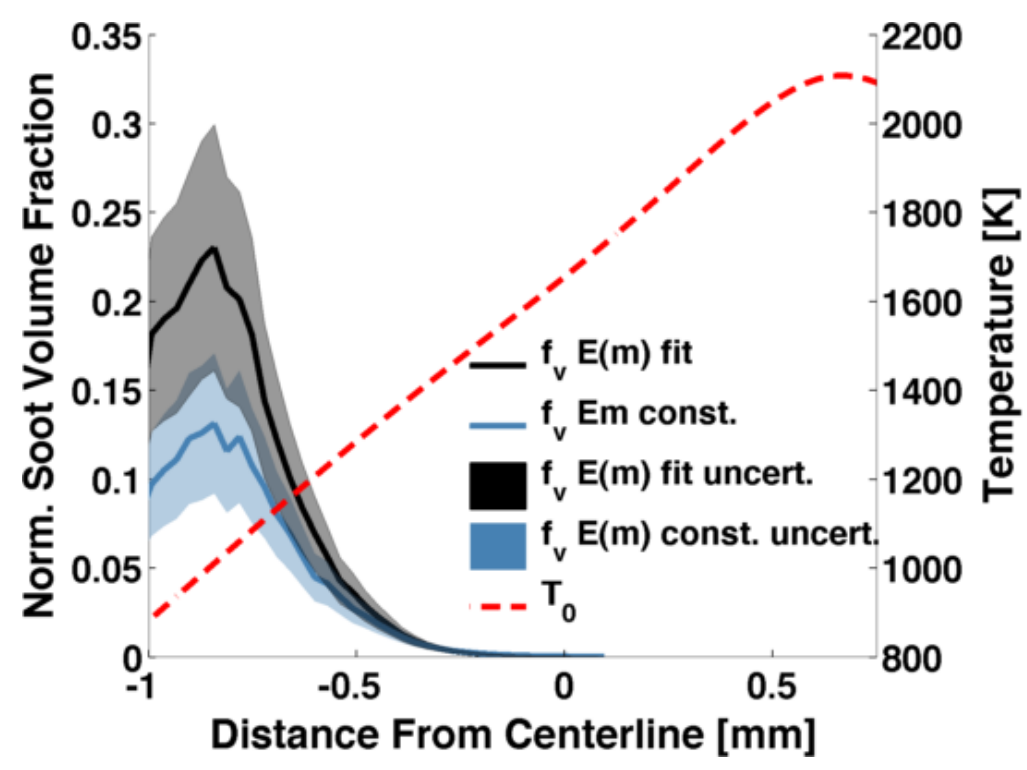

Figure 3.3: A comparison of LII determined normalized volume fraction assuming $E(m)=$ 0.24 (light blue line) with uncertainty (light blue shaded region), and $E(m)$ least squares fit (black line) with uncertainty (gray shaded region) at $1 \mathrm{~atm}, a_{l}=91 \mathrm{~s}^{-1}, X_{C_{2} H_{4}}=1.0$ with opposed flow of air.

best represent the LII physics. For other flame configurations where flame temperatures are more spatially uniform with small gradients, it may be sufficient to assume a global value for $E(m)$. However, it is suggested that a fitting procedure for $E(m)$ is always used where the uncertainties are lower than assuming a constant global value for $E(m)$ without precise knowledge of the true value.

\subsubsection{Final LII Measurement Uncertainty}

Allowing the absorption function to change spatially as a best fit to temperature anchors $E(m)$ to the results of volume fraction causing a second order effect on the uncertainties of the determined variables. With the dependency of volume fraction on the absorption function assumed and the thermal accommodation locally mixture averaged, the uncertainties for all LII determined variables are recalculated in Table 3.5. Assuming no particle aggregation and a sufficiently calibrated laser sheet fluence profile, it is notable that the uncertainty bounds for the absorption function determined by LII are lower than the range 
Table 3.5: Revised uncertainties in Laser Induced Incandescence extracted variables.

\begin{tabular}{|c|c|c|c|c|c|c|c|}
\hline \multicolumn{2}{|c|}{ 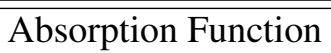 } & \multicolumn{2}{|c|}{ Volume Fraction } & \multicolumn{2}{|c|}{ Primary Particle Size } & \multicolumn{2}{|c|}{ Number Density } \\
\hline Param. & Uncert. & Param. & Uncert. & Param. & Uncert. & Param. & Uncert. \\
\hline$T_{0}$ & $18 \%$ & $T_{0}$ & $20 \%$ & $T_{0}$ & $30 \%$ & $T_{0}$ & $56 \%$ \\
\hline$\rho_{s}$ & $10 \%$ & $\rho_{s}$ & $10 \%$ & $\alpha_{T}$ & $22-32 \%$ & $\alpha_{T}$ & $38-56 \%$ \\
\hline$c_{s}$ & $10 \%$ & $c_{s}$ & $10 \%$ & $\gamma$ & $15 \%$ & $\gamma$ & $26 \%$ \\
\hline$F_{0}$ & $8 \%$ & $F_{0}$ & $8 \%$ & $\rho_{s}$ & $10 \%$ & $\rho_{s}$ & $20 \%$ \\
\hline$\eta$ & $4 \%$ & $I_{L I I}$ & $6 \%$ & $c_{s}$ & $10 \%$ & $c_{s}$ & $20 \%$ \\
\hline$\Omega$ & $4 \%$ & $\eta$ & $6 \%$ & $Q_{\text {cond }}$ & $8 \%$ & $Q_{\text {cond }}$ & $14 \%$ \\
\hline$A_{e}$ & $4 \%$ & $\Omega$ & $5 \%$ & $F_{0}$ & $6 \%$ & $F_{0}$ & $13 \%$ \\
\hline$I_{L I I}$ & $5 \%$ & $A_{e}$ & $5 \%$ & $\kappa$ & $4 \%$ & $W_{g}$ & $8 \%$ \\
\hline$\alpha_{T}$ & $3 \%$ & $\alpha_{T}$ & $5 \%$ & $W_{g}$ & $4 \%$ & $\kappa$ & $8 \%$ \\
\hline$\kappa$ & $2 \%$ & $\kappa$ & $4 \%$ & $p_{0}$ & $4 \%$ & $p_{0}$ & $8 \%$ \\
\hline$\gamma$ & $2 \%$ & $\gamma$ & $4 \%$ & $y$ & 0 & $I_{L I I}$ & $6 \%$ \\
\hline$Q_{\text {cond }}$ & $2 \%$ & $Q_{\text {cond }}$ & $4 \%$ & $W_{v}$ & 0 & $\eta$ & $6 \%$ \\
\hline$W_{g}$ & $2 \%$ & $W_{g}$ & $4 \%$ & $\alpha_{M}$ & 0 & $\Omega$ & $5 \%$ \\
\hline$p_{0}$ & $2 \%$ & $p_{0}$ & $4 \%$ & $p_{\text {ref }}$ & 0 & $A_{e}$ & $5 \%$ \\
\hline$y$ & 0 & $y$ & 0 & $\Delta H_{v}$ & 0 & $y$ & 0 \\
\hline$W_{v}$ & 0 & $W_{v}$ & 0 & $T_{r e f}$ & 0 & $W_{v}$ & 0 \\
\hline$\alpha_{M}$ & 0 & $\alpha_{M}$ & 0 & $I_{L I I}$ & 0 & $\alpha_{M}$ & 0 \\
\hline$p_{r e f}$ & 0 & $p_{\text {ref }}$ & 0 & $\eta$ & 0 & $p_{r e f}$ & 0 \\
\hline$\Delta H_{v}$ & 0 & $\Delta H_{v}$ & 0 & $\Omega$ & 0 & $\Delta H_{v}$ & 0 \\
\hline$T_{r e f}$ & 0 & $T_{r e f}$ & 0 & $A_{e}$ & 0 & $T_{r e f}$ & 0 \\
\hline Total & $26 \%$ & Total & $30 \%$ & Total & $44-50 \%$ & Total & $82-91 \%$ \\
\hline
\end{tabular}

reported in literature by more than $20 \%$. While a significant uncertainty still remains, attempting to determine the local absorption function value spatially in situ within a theoretical framework by a convergent solution between extinction and LII as opposed to applying a global nominal value can be attributed to the above reduction in uncertainty. It is also clear from Table 3.5 that relatively few parameters make up a majority of the uncertainty in LII variables. Of all the variables, the easiest to improve upon is the thermal accommodation coefficient with an effort by the scientific community to develop a more complete species-surface pair thermal accommodation database for soot as a function of gas and surface temperature both experimentally and though MD simulations with clearly documented uncertainty. This effort would go a long way in improving the confidence of thermal accommodation and LII particle sizing results. 


\subsubsection{Repeatability Uncertainty}

A repeatability study was conducted to determine the magnitude of repeatability uncertainty on LII extracted measurements of volume fraction $\left(f_{v}\right)$, primary particle diameter $\left(d_{p}\right)$, and number density $(N)$. A full repeatability study of all LII collected data would have been cost and time prohibitive due to the time required to conduct individual measurements, the cost of high purity gases, and the large dataset collected over a range of pressures, strain rates, and fuel-oxidizer compositions. Instead, three samples were collected at 8.45 atm under different fuel-oxidizer compositions and soot loadings:

(a) Three measurements at a local strain rate of $163 \mathrm{~s}^{-1}, 0.46 \mathrm{C}_{2} \mathrm{H}_{4}+0.54 \mathrm{~N}_{2}, 0.1933 \mathrm{O}_{2}$ $+0.8067 \mathrm{~N}_{2}$.

(b) Three measurements at a local strain rate of $163 \mathrm{~s}^{-1}, 0.42 \mathrm{C}_{2} \mathrm{H}_{4}+0.58 \mathrm{~N}_{2}, 0.1765 \mathrm{O}_{2}$ $+0.8235 \mathrm{~N}_{2}$.

(c) Three measurements at a local strain rate of $186 \mathrm{~s}^{-1}, 0.3982 \mathrm{C}_{2} \mathrm{H}_{4}+0.0046$ toluene + $0.5972 \mathrm{~N}_{2}, 0.1681 \mathrm{O}_{2}+0.8319 \mathrm{~N}_{2}$.

Samples at 8.45 atm were chosen because they represented challenging experiments to collect and thus good predictors for conservative repeatability bounds. Figures 3.4 a), b), and c) show the results of volume fraction repeatability for the same three samples described above. The solid black lines show normalized volume fraction profiles of individual measurements from the samples. The point of maximum flame temperature represented by the vertical dashed red line is shown for spatial reference. Points left of the maximum flame temperature indicate the fuel rich region while points to the left indicate the oxygen rich region of the counterflow field. The point $z=0$ represents the centerline between the two counterflow nozzles. In all cases soot is formed in the fuel rich region. Good agreement is exhibited concerning repeatability between the individual measurements in each sample. The blue shaded area in the figures shows the repeatability band one standard deviation 


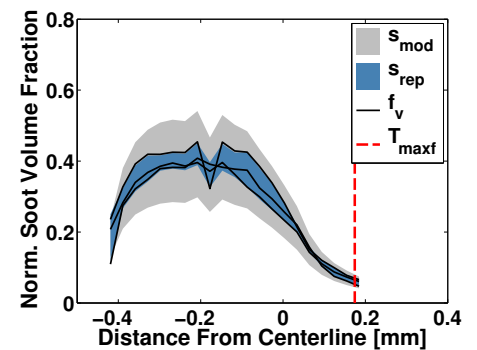

(a)

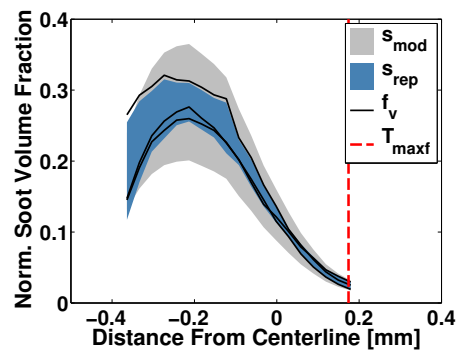

(b)

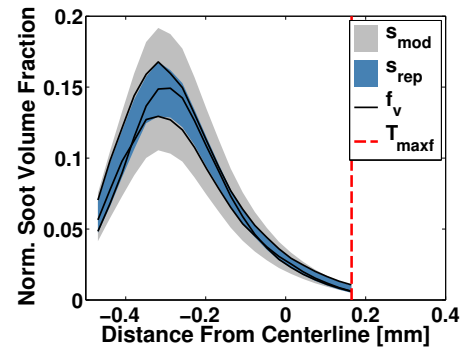

(c)

Figure 3.4: Volume fraction repeatability for samples a), b), and c) described above. The figure includes individual normalized volume fraction measurements (black solid line), point of maximum flame temperature (red dashed line), repeatability standard deviation band (blue shaded region), and standard model uncertainty band (grey shaded region).

above and below the mean of the sample. The standard LII measurement uncertainty calculated in Chapter 3.1.5 is also shown for comparison by the gray shaded region. Overall the results show good agreement in the magnitude of repeatability uncertainty $(10-15 \%)$ between the three samples. For all cases the relative standard LII measurement uncertainty is larger than the repeatability uncertainty by about a factor of two for the majority of the volume fraction profile. The extreme edges do show increased relative uncertainty due to decreased volume fraction and an increase in incandescent signal to noise at the edges.

Figures $3.5 \mathrm{a}$ ), b), and c) show the results of primary particle diameter repeatability for the three repeatability samples described above. The same plotting designations apply as above with the solid black lines showing the normalized primary particle diameter profiles of individual measurements from the samples. The individual samples are all in good agreement with a magnitude of repeatability uncertainty around $10-15 \%$ on average. In this case the relative standard LII measurement uncertainty is much larger than the repeatability uncertainty by a factor of four to five at all but particle inception near the peak particle temperature. Larger repeatability uncertainty of $30 \%$ or greater at small particle size near particle inception at the peak flame temperature is observed likely due to signal to noise defficiency near the edges of the incandescent signal profile. 


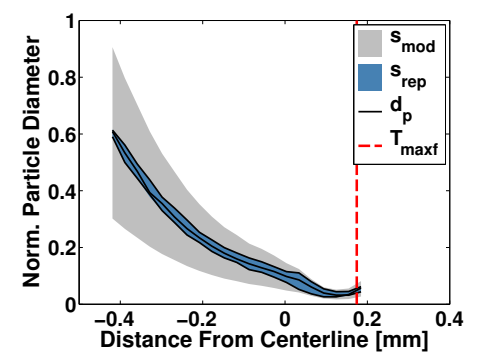

(a)

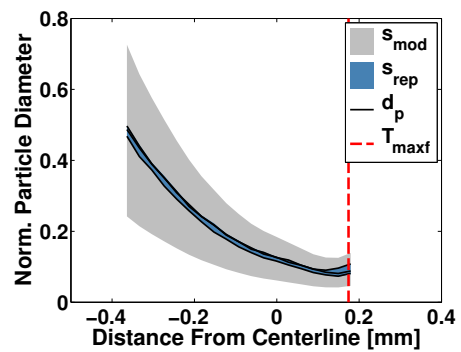

(b)

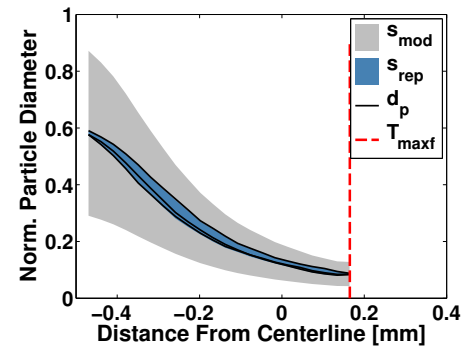

(c)

Figure 3.5: Primary particle diameter repeatability for samples a), b), and c) described above. The figure includes individual normalized particle diameter measurements (black solid line), point of maximum flame temperature (red dashed line), repeatability standard deviation band (blue shaded region), and standard model uncertainty band (grey shaded region).

Figures 3.6 a), b), and c) show the results of number density repeatability for the three repeatability samples described above. The solid black lines show the normalized number density profiles of individual measurements from the samples. While for soot volume fraction and primary particle diameter, the repeatability uncertainty is consistently less than model uncertainty, in some cases, e.g. Fig. 3.6 a), the repeatability uncertainty for number density is large and on the order of model uncertainty especially at the peak of the profile. This is expected given the equation for calculating number density $N=6 f_{v} / \pi d_{p}^{3}$ makes use of both the measured volume fraction and primary particle diameter. The expected peak of the number density profile coincides around particle inception near the peak flame temperature where the relative repeatability uncertainty of volume fraction and primary particle diameter is at a maximum at the edge. Thus the repeatability uncertainty around maximum number density is large. In any case, it is shown in Fig. 3.6 a)-c) that number density changes by more than two orders of magnitude for the test samples considered. In fact peak number density changes by upwards of four to five orders of magnitude across all pressure, residence time, and fuel-oxidizer compositions considered. Hence useful trends in number density can still be garnered with a large repeatability and model uncertainty.

For soot volume fraction and primary particle size, model uncertainty remains the dominant uncertainty for LII measurements even after the uncertainty improvements to the mea- 


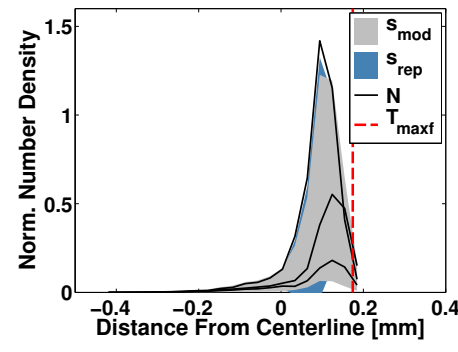

(a)

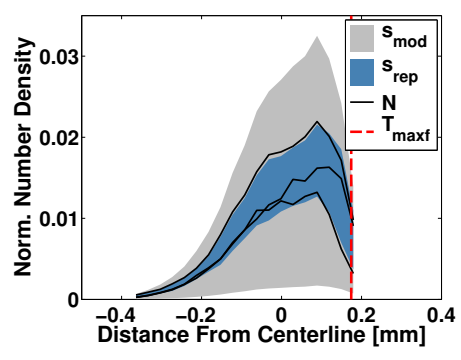

(b)

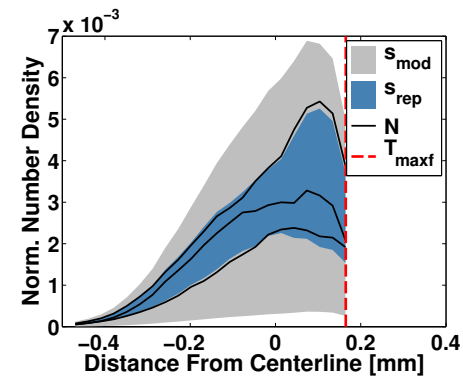

(c)

Figure 3.6: Number density repeatability for samples a), b), and c) described above. The figure includes individual normalized number density measurements (black solid line), point of maximum flame temperature (red dashed line), repeatability standard deviation band (blue shaded region), and standard model uncertainty band (grey shaded region).

surement procedure proposed in Chapter 3.1.5. However, a reduction in uncertainty on the local ambient gas temperature or thermal accommodation to levels more in line with the other documented measurement uncertainties would quickly result in repeatability and LII measurement uncertainties of the same magnitude. In this case further improvements to the accuracy of the measurement system would be challenging.

\subsubsection{Local Gas Properties and Other Uncertainties}

The uncertainty in local gas temperature, heat capacity ratio, thermal conductivity, and mixture-averaged molecular weight is all dominated by spatial uncertainty between experiments and computations of approximately $0.15 \mathrm{~mm}$. A method of exactly anchoring the LII data and modeled data to a precise spatial location would remove most of the error on these quantities and greatly improve LII results. If a different burner configuration were used with a more certain and less varying temperature, a decrease in uncertainty would be realized. However, uncertainty due to aggregation would be greatly increased from an inability to control residence time. Reducing the local temperature and thermal accommodation uncertainties under more well described conditions (assuming $\sim \pm 50 \mathrm{~K}$ and $\sim 15 \%$ respectively) would reduce the uncertainty on $E(m), f_{v}, d_{p}$, and $N$ to approximately $18 \%$, $21 \%, 26 \%$, and $48 \%$, respectively. This is considered an approximate lower limit for the 
total standard uncertainty of LII extracted variables.

\subsection{Particle Image Velocimetry Uncertainty}

Particle image velocimetry was employed to measure the two dimensional velocity profile between the counterflow nozzles in order to characterize the flow residence time critical for accurate chemical-kinetic simulation. The relatively large velocity samples collected at small spatial increments (50-100 microns) resulted in well defined velocity profiles both in the axial and radial directions. The local strain rate was chosen as the measure to represent flow residence time. The local strain rate was determined via a second order polynomial regression fit to the mean oxidizer stream axial velocity along the axial centerline from oxidizer nozzle exit to the thermal mixing layer of the flame. A generic example of the regression bounds is given in Fig. 3.7. The fuel nozzle exit is located on the left at $6 \mathrm{~mm}$ and the oxidizer nozzle on the right at $+6 \mathrm{~mm}$. Indications of the thermal mixing layer and flame location are shown by the peak in the profile to the right of the centerline. The black line indicates the regression bounds for calculating the local strain rate which also includes the red region. The maximum velocity gradient on the air side just prior to the thermal mixing layer was identified as the characteristic local strain rate for each experiment and is illustrated by the region in red. Determination of uncertainty in the local strain rate was conducted using the procedure outlined in the NIST/SEMATECH eHandbook of Statistical Methods with additional procedures developed to take into account the estimated uncertainty of each velocity point measured with PIV. The procedure is as follows.

\subsubsection{Measurement Uncertainty}

PIV requires two images of a seed particle laden flow taken a known time interval apart. Two laser pulses illuminate the particles while the two images record the particle spatial lo- 


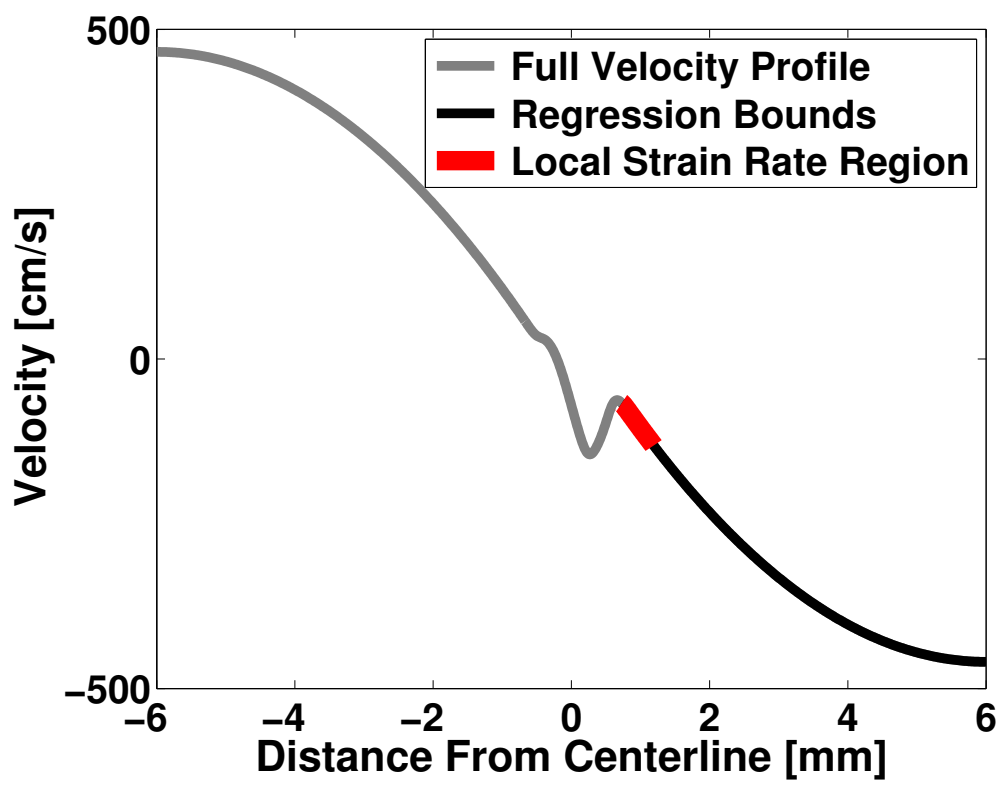

Figure 3.7: A generic example of the full reacting axial velocity profile between the counterflow nozzles along the axial centerline.

cations at two different times. The procdecure also requires an accurate spatial calibration to determine local velocities from the distance the particles have traveled over the known time i.e. $v=\Delta z / \Delta t$. A sample size of five hundred was used for a single PIV experiment to calculate the average velocity of the test. The average velocity is used for all error analysis, the validity of which was determined previously [35]. Each experiment was repeated several times to provide a sample of average velocity measurements. The mean and standard deviation of the instantaneous five hundred image velocity population measured at a spatial location by PIV is unknown. It is therefore necessary to estimate the standard deviation of the population $\sigma$ using the standard deviation of the sample $s$. Additional sources of measurement uncertainty were identified and included in the uncertainty analysis at each measurement point in the flow field. Uncertainty in the spatial calibration was estimated based on the known outer diameter of the inner co-annular oxidizer nozzle. The uncertainty in the time between laser pulses was taken from hardware documentation. An inability of large seed particles to perfectly follow the local gas velocity must also be considered. Uncertainty in particle velocity due to flow slip was calculated from literature as $0.67 \%$ due 
to 1 micron particles used for seeding to achieve adequate particle illumination. The low error is a result of using low velocity laminar flows. High-speed turbulent flows can exhibit large slip velocity errors. The equation for calculating relative uncertainty due to particle slip velocity is given by $[119,120]$

$$
\Delta v_{s l i p}=\frac{d_{s}^{2} \rho_{\text {seed }} \zeta}{18 \mu}
$$

Where $d_{s}$ is the seed particle diameter, $\rho_{\text {seed }}$ the seed particle density, $\zeta$ the maximum velocity gradient expected in the flow field, and $\mu$ the fluid dynamic viscosity. A summary of estimated PIV measurement errors are given in Table 3.6.

Table 3.6: Estimated PIV measurement uncertainty.

\begin{tabular}{lll}
\hline \hline Variable & Description & Uncertainty \\
\hline$\Delta z, r$ & Spatial coordinates & $1.1 \%$ \\
$\Delta t$ & Time between laser pulses & $10 \mathrm{~ns}$ (negligible) \\
$\Delta v_{\text {slip }}$ & Particle velocity uncertainty due to slip & $0.67 \%[119,120]$
\end{tabular}

Error propagation was conducted using all applicable measurement errors. The root sum square (RSS) error method was first used to estimate the propagation of error from the uncertainty identified in the variables $z$ and $t$ used to calculate velocity from the equation $v=\Delta z / \Delta t$ assuming no covariances. The relative uncertainty $(\Delta)$ of each variable was used to obtain

$$
\Delta v_{P I V}^{2}=\left(\Delta z \frac{\delta v_{P I V}}{\delta z}\right)^{2}+\left(\Delta t \frac{\delta v_{P I V}}{\delta t}\right)^{2}
$$

Which reduces to the more commonly used equation for propagation of error [121],

$$
\Delta v_{P I V}=\sqrt{\Delta z^{2}+\Delta t^{2}}
$$

The uncertainty of PIV derived velocity is dominated by the spatial calibration uncertainty and is assumed to have a standard deviation of $1.1 \%$ from a uniform distribution. Errors from particle slip and the velocity calculation were then combined with the standard sample 
uncertainty in an RSS error equation to estimate a total standard uncertainty at each spatial measurement point

$$
\Delta v_{t o t}^{2}=\left(\Delta v_{s l i p} \frac{\delta v_{t o t}}{\delta v_{s l i p}}\right)^{2}+\left(s \frac{\delta v_{t o t}}{\delta s}\right)^{2}+\left(\Delta v_{P I V} \frac{\delta v_{t o t}}{\delta v_{P I V}}\right)^{2}
$$

or

$$
\Delta v_{t o t}=\sqrt{\Delta v_{\text {slip }}^{2}+s^{2}+\Delta v_{P I V}^{2}}
$$

The total error estimate was between 6-25\% at each measurement point and was dominated by the sample standard deviation.

\subsubsection{Uncertainty of the Regression Model}

It is worth noting that a parabolic regression fit could have been calculated for one experiment from the sample mean velocity at each spatial location and the uncertainty of the regression coefficients calculated using a linear least-squares approach [122]. A regression on this data, however, would not accurately represent the sample uncertainty since it does not take into account additional measurement errors and the sample uncertainty at each spatial location. A Monte Carlo simulation was utilized to account for these errors by reproducing a random dataset from a normal distribution with a mean of the measured sample velocity and a standard deviation of the total standard uncertainty estimated by Eq. 3.7 at each spatial location. A sample of five hundred randomly generated velocity curves were model fit using a parabolic linear least-squares regression and standard error intervals were calculated for all three polynomial coefficients using Matlab and the regress function [123]. The spatial location corresponding to the local strain rate and the standard deviation of the first and second degree coefficients were input into the propagation of error equation to estimate the uncertainty in the local strain rate $\left(\Delta a_{l}\right)$ measured from the regression of the Monte Carlo generated sample for each independent experiment $k$. The equation is given 
by

$$
\Delta a_{l, k}=\sqrt{\left(2 z \Delta \beta_{2, k}\right)^{2}+\Delta \beta_{1, k}^{2}}
$$

Where $\Delta \beta_{1, k}$ and $\Delta \beta_{2, k}$ are the uncertainties of the first and second-order coefficients of the $k$-th experiment. These estimates serve as the most appropriate standard uncertainty for the local strain rate. Depending on the degrees of freedom of the regression and the quality of the sample data, the standard uncertainty estimate on the local strain rate varies from between approximately 8 and $25 \%$ and generally increases with increasing pressure. The increase is primarily due to optical distortion issues from flame heat release and particle clumping with increasing pressure. The final standard uncertainty in the local strain rate from each independent measurement due to regression was calculated via the propagation of error equation given by

$$
\Delta a_{l, r e g}=\sqrt{\frac{1}{J} \sum_{k=1}^{J} \Delta a_{l, k}^{2}}
$$

\subsubsection{Repeatability}

Local extinction strain rate samples were collected from a number of repetitions for each fuel-air composition, strain rate, and pressure case considered. A temporal uncertainty was calculated and included in the error analysis to account for deviation across samples and the temporal errors due to environmental and experimental process control uncertainties. The level-1 repeatability standard deviation $\Delta a_{l, r e p}$, was calculated by

$$
\Delta a_{l, r e p}=\sqrt{\frac{1}{J-1} \sum_{k=1}^{J}\left(a_{l, k}-\bar{a}_{l}\right)^{2}} .
$$

Where $J$ is the number of repetitions taken on the sample in question, $a_{l, k}$ the local strain rate of the $k$-th repetition, and $\bar{a}_{l}$ the mean over all repetitions [121]. 


\subsubsection{Total Uncertainty}

The total regression uncertainty and the temporal standard deviation were combined via the propagation of error equation

$$
\Delta a_{l, t o t}=\sqrt{\Delta a_{l, r e g}^{2}+\Delta a_{l, r e p}^{2}}
$$

This method effectively provides a high level of confidence to all variables of interest. Standard error estimates for local strain rates at pressures from 1 atm to $14 \mathrm{~atm}$ varies between 8 and $25 \%$ with results at pressures of $20 \mathrm{~atm}$ and $30 \mathrm{~atm}$ higher around $30-65 \%$ due to issues of optical distortion and particle clumping as previously mentioned. A 95\% confidence interval can be estimated by multiplying the total standard uncertainty by 2 . Detailed $95 \%$ confidence interval error analysis was conducted and confirmed the validity of the simple factor of 2 estimation. 


\section{Chapter 4}

\section{Experimental Results}

\subsection{Transmission Electron Microscopy Results}

Laser induced incandescence provides insight into several key soot formation and growth characteristics including soot volume fraction, number density, effective particle size, and some hints into soot morphology from optical absorption. Yet several unresolved questions remain that influence necessary inputs to a detailed LII heat transfer model. Most importantly: What particle size distribution assumption is appropriate? If soot aggregates are present, what are the fractal dimensions unique to the flame being measured and what aggregate distribution assumption should be made? The thermophoretic sampling procedure for soot outlined in Chapter 2.6 coupled with transmission electron microscopy (TEM) analysis offers answers to some of these unresolved questions. Additional clues into soot morphology unique to the counterflow diffusion flame configuration can also be assessed. Due to the thin nature of counterflow flames at high pressure and rapid changes due to soot formation and growth in a thin sooting region, precise spatial sampling of soot is not possible. The sampling width of the thermophoretic probe $(1 \mathrm{~mm})$ is on the order of the soot formation region width $(\sim 0.5-1 \mathrm{~mm})$ and thus the entire sooting region is sampled on one grid. We can however still determine several key variables of global relevance to the high 


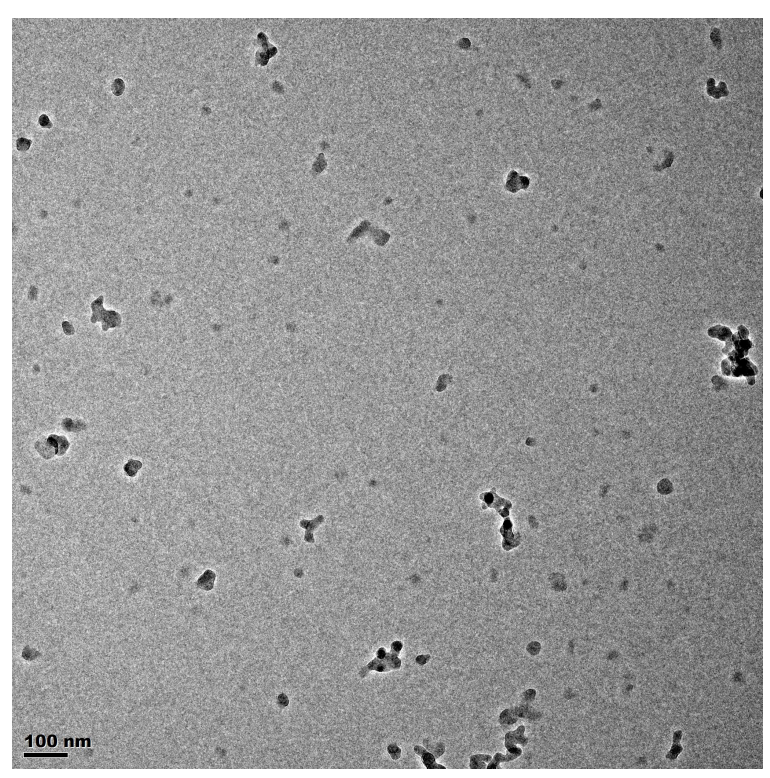

(a) TEM image of a sample of aggregates.

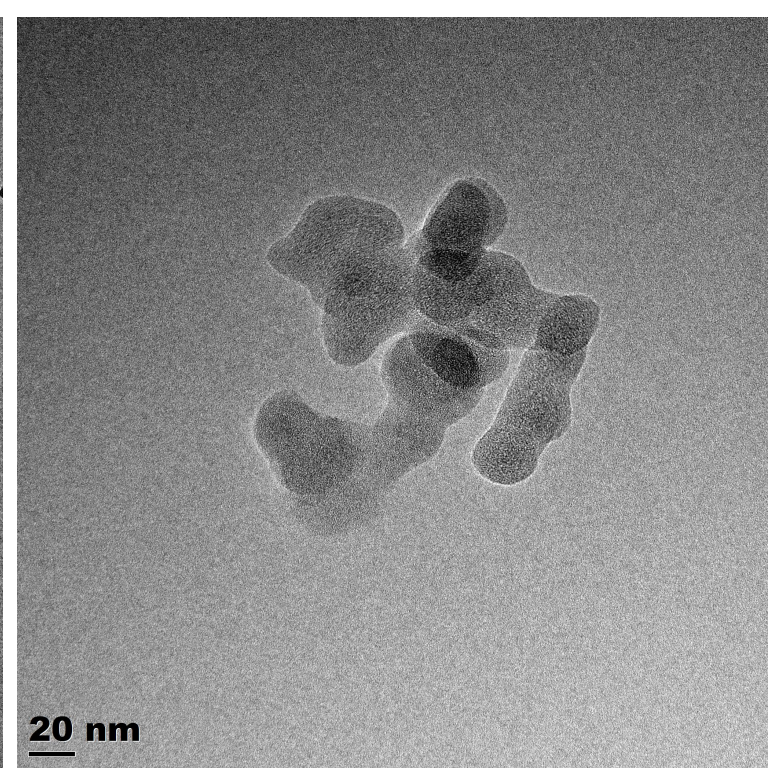

(b) TEM image of a single aggregate.

Figure 4.1: TEM aggregate images at a pressure of $8.45 \mathrm{~atm}, a_{l}=163 \mathrm{~s}^{-1}, X_{C_{2} H_{4}}=$ $0.46+X_{N_{2}}=0.54$ with opposed flow $X_{O_{2}}=0.1933+X_{N_{2}}=0.8067$.

pressure counterflow flame configuration. Most notably particle size distribution, aggregate size distribution, and aggregate dimensions along with some clues into the morphology of soot can be determined.

\subsubsection{Fractal Aggregate Dimensions}

In most instances, the residence time in the non-premixed counterflow flame was too short to induce significant aggregation of soot from the fuel and oxidizer compositions used in this study. A notable exception was data collected at $8.45 \mathrm{~atm}$. Thermophoretic samples of soot were collected from the counterflow burner at a pressure of $8.45 \mathrm{~atm}, a_{l}=163 \mathrm{~s}^{-1}$, $X_{C_{2} H_{4}}=0.46+X_{N_{2}}=0.54$ with opposed flow $X_{O_{2}}=0.1933+X_{N_{2}}=0.8067$. A survey of TEM images like that illustrated in Fig. 4.1 a) show soot particles of varying sizes from primary particles to aggregates containing on average $20-30$ primary particles and up to approximately 60 in some instances. No aggregates making up more than 60 primary particles were observed in any TEM images. While the residence times are not known exactly as discussed previously, correlations with LII results indicate the TEM images represent 
residence times on the order of $10 \mathrm{~ms}$. The aggregates formed using the counterflow burner appear generally smaller than those formed in a co-flow diffusion burner [39] or premixed flat flame burner [24] at similar pressures and using the same $\mathrm{C}_{2} \mathrm{H}_{4}$ fuel making it a good candidate for focusing on early soot nucleation and growth.

A relation $[75,91]$ is often used in LII $[76,89,102,124]$, and combined extinction and scattering [125-127] literature to relate the number of primary particles in an aggregate $N_{p}$ to the radius of gyration of the aggregate $R_{g}$ and the average primary particle radius $d_{p}$ using a fractal prefactor $k_{f}$ and fractal dimension $D_{f}$. The relation was introduced in Chapter 2.4 and is given by

$$
N_{p}=k_{f}\left(\frac{2 R_{g}}{d_{p}}\right)^{D_{f}}
$$

An analysis of images of individual aggregates like that shown in Fig. $4.1 \mathrm{~b}$ ) using the procedure pioneered by Brasil et al. [75] allowed for an estimation of the aggregate dimensions unique to the high pressure counterflow burner. Figure 4.2 shows measurements of the number of primary particles per aggregate $N_{p}$ plotted versus maximum aggregate length $L$ and average primary particle diameter $d_{p}$. Given the relation $N_{p}=k_{L}\left(L / d_{p}\right)^{D_{f L}}$ for projected fractal properties [75], the data from Fig. 4.2 was regression fit resulting in prefactor parameter $k_{L}=1.64$ and slope parameter $D_{f L}=1.41$. Using the supporting relations from Brasil et al. $D_{f}=D_{f L}$ and $k_{f}=k_{L}(1.5)^{D_{f}}$ the aggregate dimensions for the high pressure counterflow burner used in this study were calculated as $D_{f}=1.41$ and $k_{f}=2.9$. Repeating the measurements resulted in similar values. Determination of these burner specific fractal aggregate dimensions allows for the potential application of elastic light scattering (ELS) data to determine $d_{p}$ and $N_{p}$ from formed aggregates. The unique dimensions also shed light on a structure noticeably different than soot produced from other burners. The fractal dimension $D_{f}$ is lower than the 1.6-1.9 range generally cited in literature [91] for premixed burners. More importantly the fractal prefactor $k_{f}=2.9$ is in the range of values reported in literature between $k_{f}=1.62$ [125] to $k_{f}>4$ [91] with recent LII and scattering literature $[89,128,129]$ generally assuming nominal values around 


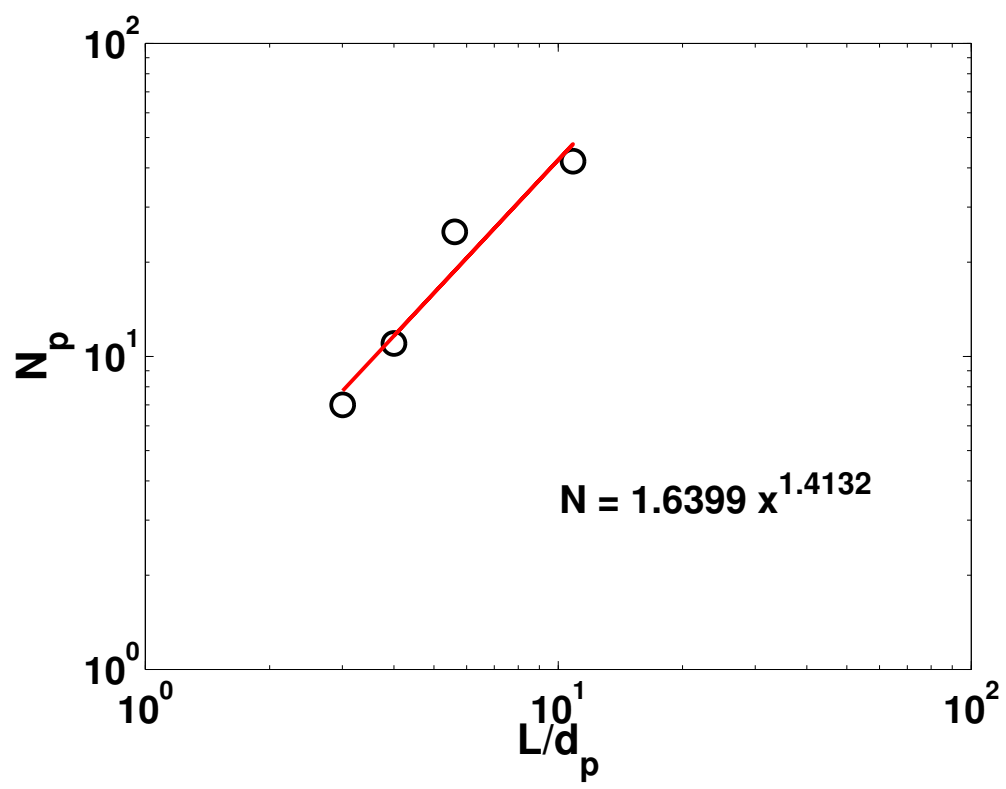

Figure 4.2: Number of primary particles per aggregate $N_{p}$ as a function of $L / d_{p}$ where $L$ is the maximum aggregate length and $d_{p}$ the soot primary particle diameter.

$D_{f}=1.7$ and $k_{f}=1.9$. A larger value for $k_{f}$ is representative of greater overlap between primary particles and lower effective surface area available for heat loss or surface reactions. The overlap between primary particles is clearly evident in Fig. 4.1 b). A relation was suggested by Brasil et al. [75]

$$
S_{a} / S_{a\left(C_{o v}=0\right)}=1-1.3 C_{o v}(1-1 / N)
$$

correlating the reduction in surface area of an aggregate relative to an aggregate with point contact between primary particles (no overlap). Given a relation for the overlap parameter from Chapter 2.3.7, $C_{o v}=-0.0735 k_{f}^{2}+0.5399 k_{f}-0.6398$, the available surface area of aggregates in this study is reduced to only $63 \%$ of the total (no overlap) surface area for an aggregate with 10 primary particles. This reduction in available surface area for heat loss or surface reactions is significant and illustrates that the soot particles produced in a high pressure counterflow burner are densely packed. This is suggestive of early primary particle coagulation combined with subsequent surface growth [14]. The dense clusters may pose additional challenges for combined scattering and LII determination of primary 
particle sizes as the definition of primary and coagulated particles becomes blurred due to the closely packed dimensions.

\subsubsection{Primary Particle Diameter and Aggregate Size Distributions}

Additional analysis of TEM images allows for determination of distribution parameters for $d_{p}$ and $N_{p}$. As previously mentioned, spatially resolved particle and aggregate sizes cannot be measured in high pressure counterflow flames for direct comparison with LII due to lack of precise knowledge of the sampling location. Thus calculated median values are relevant to only one unknown spatial location, and are not particularly useful for comparison to other measurements. However, the distribution shapes and widths calculated at one position are assumed relevant to all locations within the sooting region without definitive evidence in literature to suggest otherwise. Normalized lognormal distributions for $d_{p}$ and $N_{p}$ are shown in Fig. 4.3 a) and b) respectively and result in good fits to the data collected from TEM images. The median primary particle diameter is in agreement with LII results at longer particle residence time but no precise location can be determined. The standard deviation of the natural logarithm of the diameter distribution $\sigma_{d_{p}, g}=1.2$ is in agreement with other results in literature $[24,78,130]$ using several burner configurations. The bimodal diameter distribution cited by many $[13,48,88]$ to occur in flames was not observed here. Individual soot primary particle diameters were determined from images of single aggregates. Incipient soot on the order of $5 \mathrm{~nm}$ was unresolvable in the background noise of more expansive images representing a larger area and more aggregates. Thus the first peak of the bimodal distribution due to recent soot inception was not captured but is acknowledged to coincide with soot inception as a commonly observed phenomenon. The lognormal distribution fit for $N_{p}$ resulted in $\sigma_{N_{p}, g}=2.58$ on the low end of the range of values reported in literature ( [128] and citations within) using several flame configurations. This may be due to the relatively small aggregates observed from TEM images where the distribution width may increase with increasing aggregate size and additional time for new 


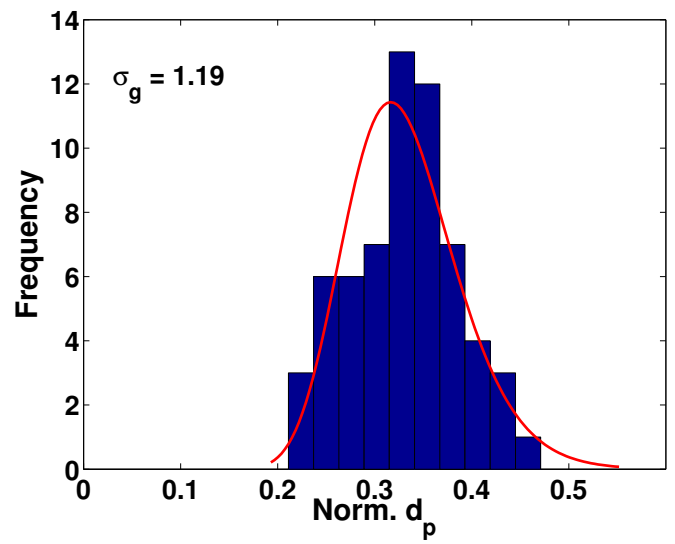

(a) Normalized soot primary particle sizes counted from TEM images.

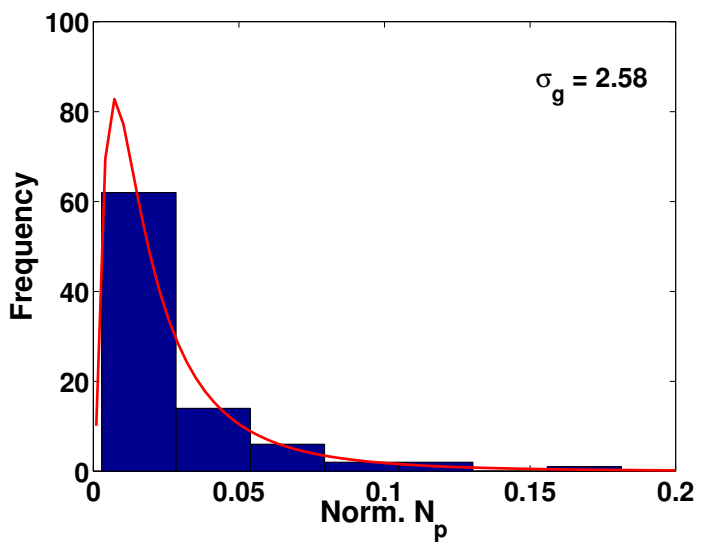

(b) Normalized number of primary soot particles per aggregate counted from TEM images.

Figure 4.3: Histogram of a) normalized primary particle diameter and b) normalized number of primary soot particles per aggregate counted from TEM images. Lognormal fits to the data are provided with the median and standard deviation of the natural logarithm of the distributions.

soot nucleation, growth, and coagulation. In any case, the narrow distributions of $d_{p}$ and $N_{p}$ observed in this study provide the option to make some simplifying assumptions in LII modeling with the counterflow configuration. Assumptions of monodisperse particle diameter and aggregate distributions were tested against the TEM determined distribution parameters. The differences to extracted variables $f_{v}, d_{p}$, and $N$ were generally less than 5-10\%. Taking into consideration the uncertainties on LII derived quantities on the order of $30-100 \%$, monodisperse distribution assumptions should be considered satisfactory and are utilized here to reduce data processing time.

\subsubsection{Soot Particle Morphology}

Little is known about the morphological structure of soot formed in counterflow flames and even less so at elevated pressure. The majority of analysis has been conducted using soot samples collected from premixed or co-flow diffusion burners. Several key observations have been deduced providing clues into optical properties, structure, and formation mechanisms. Kim et al. examined the effect of changing particle size with pressure 
and fuel/oxidizer concentrations. Clear trends of decreasing primary particle size with increased oxidative attack was observed. An increase in primary particle size was attributed to increasing pressure due to greater species concentrations involved in soot growth and increased residence time [39]. Heer and Ugarte [131] and Kimura et al. [132] used electric arc and hot filament fuel pyrolysis respectively to form soot samples and examined the effect of temperature on formation, annealing, absorption features, and structure. Increasing formation temperature was found to decrease carbon grain size and change absorption features. Infrared spectroscopy was used to determine bond structure where an increase in $\mathrm{sp}^{2}$ hybridized bonds were evident with increasing temperature corresponding to a decrease in grain size [132]. After formation, increased heat treatment temperature and time was found to dramatically increase soot graphitization [131]. Michelsen et al. [13] investigated soot formed from a premixed McKenna burner and reported TEM images of soot aggregates with noticeable graphitic structure. This was compared to the same soot irradiated with laser energy at high fluence. Noticeable changes to the long range ordered structure of the carbon layers was observed indicating vaporization and recombination to form the new structure. Near-edge x-ray absorption fine structure (NEXAFS) spectroscopy of the soot samples suggested noticeable changes in bond structure from over $70 \% \mathrm{sp}^{2}$ hybridized bonding for non irradiated soot indicating a graphitic structure to $21 \%$ at high fluence indicating a more amorphous arrangement. Recent evidence has been building indicating that soot formed under some conditions may exhibit a liquid like structure. TEM, infrared spectroscopy, and mass spectrometry data analyzed of soot collected from a premixed $\mathrm{C}_{2} \mathrm{H}_{4}-\mathrm{O}_{2}$-Ar flat flame burner by Wang and colleagues ( [6] and references within) indicates soot possessing an aromatic core and aliphatic liquid like shell. The results suggest new and unique soot growth mechanisms not yet entirely understood by the scientific community.

This author is unaware of any reported data regarding soot sampled from high pressure counterflow flames and analyzed by TEM or other analysis techniques. The counterflow 


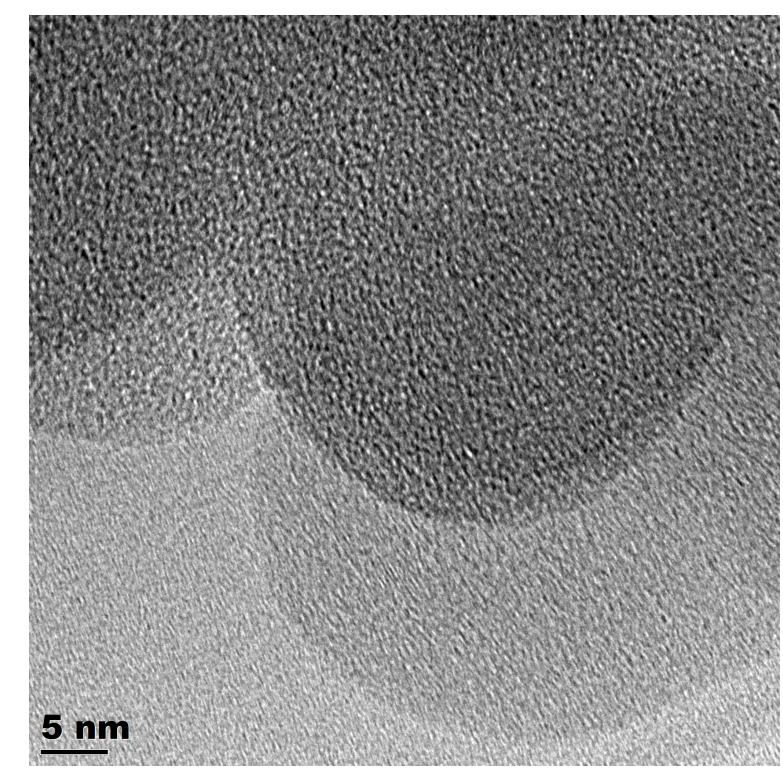

(a) TEM image of amorphous structure.

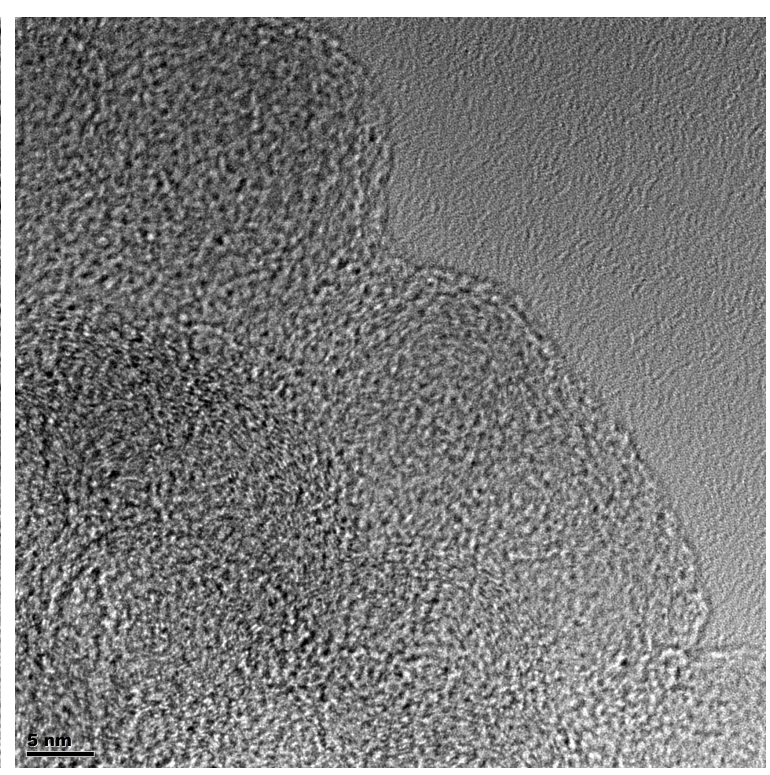

(b) TEM image of ordered layer structure.

Figure 4.4: High resolution TEM images of soot structure at a pressure of $8.45 \mathrm{~atm}, a_{l}=$ $163 \mathrm{~s}^{-1}, X_{C_{2} H_{4}}=0.46+X_{N_{2}}=0.54$ with opposed flow $X_{O_{2}}=0.1933+X_{N_{2}}=0.8067$.

burner offers a unique set of flame and flow field characteristics by which soot nucleation and growth mechanisms can be tested and explored. Figure 4.4 shows magnified views of the same soot samples shown in Fig. 4.1 above. The particles clearly exhibit a mostly randomized amorphous structure indicating interspersed $\mathrm{sp}^{2}$ and $\mathrm{sp}^{3}$ hybridized bonds. Slight indications of layered graphitic structure are observed in some images like Fig. 4.4 b) but are nonexistent in others like Fig. 4.4 a). When the fine-layered structure is observed it appears more toward the inner region of the particles in agreement with a greater probability of annealing in the high temperature region of the counterflow flame where soot inception and early growth occurs to form small primary particles. Particle inception and growth through a hydrogen abstraction $\mathrm{C}_{2} \mathrm{H}_{2}$ addition (HACA) mechanism has also been suggested to form a more graphitic like structure [14]. The HACA mechanism is widely considered a governing inception and growth mechanism at high temperature $[6,25,48,133]$. With the flame sitting on the oxidizer side, particles are initiated near the high temperature region primarily due to fuel pyrolysis with a residence time of approximately $4 \mathrm{~ms}$ in the high temperature region $\sim 2000-1600 \mathrm{~K}$. Nascent particles are then convected down a strong 
temperature gradient towards the fuel rich side of the flow field and remain in this region for approximately 5-10 ms at low temperatures in the range $1500-1000 \mathrm{~K}$. A significant portion of soot growth appears to occur at lower temperatures and observed graphitization is not prevalent. Neither do the particles exhibit the solid core and liquid shell structure observed from soot produced in some premixed flames [6] but instead appear uniformly distributed in their structure. This indicates that even though the temperature is low in the soot growth region, the mechanism for growth is likely not dominated by aliphatic surface reaction mechanisms. Instead a low temperature polycyclic aromatic hydrocarbon (PAH) surface growth mechanism is likely the key driver for this burner configuration $[25,48]$. PAH surface growth is considered less likely to form a long range ordered graphitic structure due to the range of sizes and orientations when bonding with the soot surface [14]. The hypothesis is in accordance with observations.

\subsection{Soot Particle Temperature}

Figure 4.5 shows the measured peak particle temperature from two-color pyrometry (solid red line) and the numerically calculated local gas temperature profile (dashed red line). The fuel flow originates from the left boundary $(z=-L / 2)$ and the oxidizer from the right boundary $(z=+L / 2)$ resulting in a fuel rich region in the negative $z$-direction, and an oxidizer rich region in the positive $z$-direction. $L$ is the nozzle separation where the $z=0$ point is the centerline between the two nozzles. The stoichiometric flame location is at $z=0.23 \mathrm{~mm}$ for $4.065 \mathrm{~atm}$ and $z=0.14 \mathrm{~mm}$ for $14 \mathrm{~atm}$ where the mixture fractions are $Z_{s t}=3.46$ and $Z_{s t}=1.59$ respectively. Profiles for a pressure of $4.065 \mathrm{~atm}, X_{C_{2} H_{4}}=0.5+X_{N_{2}}=0.5, X_{O_{2}}=0.21+X_{N_{2}}=0.79, a_{l}=119 \mathrm{~s}^{-1}$ and $a_{l}=408 \mathrm{~s}^{-1}$ in a) and b) and a pressure of $14 \mathrm{~atm}, X_{C_{2} H_{4}}=0.41+X_{H e}=0.59$, $X_{O_{2}}=0.1723+X_{H e}=0.8277, a_{l}=135 \mathrm{~s}^{-1}$ and $a_{l}=190 \mathrm{~s}^{-1}$ in c) and d) are presented. In addition, the numerically calculated and normalized soot precursor profiles of 

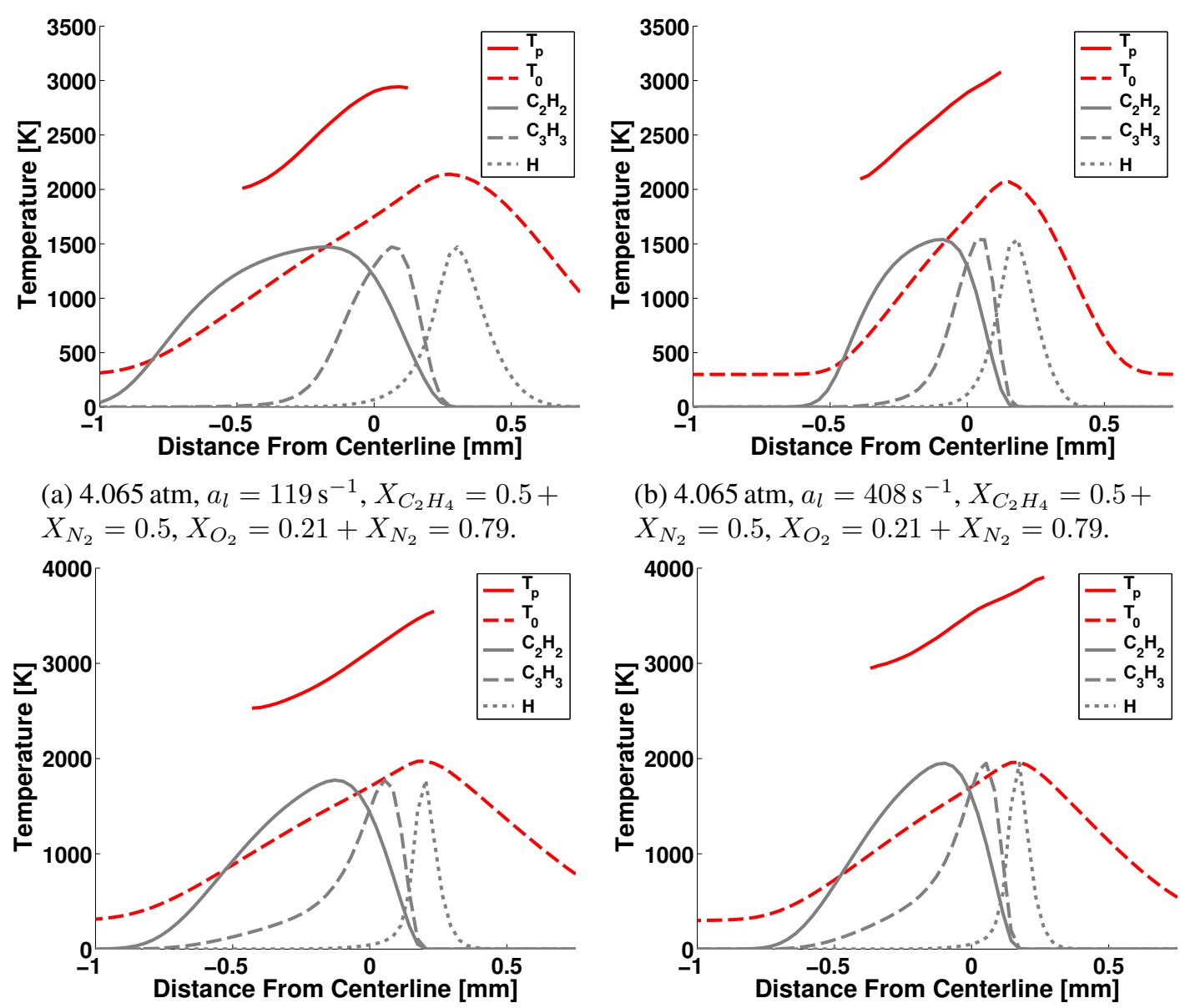

(b) $4.065 \mathrm{~atm}, a_{l}=408 \mathrm{~s}^{-1}, X_{C_{2} H_{4}}=0.5+$ $X_{N_{2}}=0.5, X_{O_{2}}=0.21+X_{N_{2}}=0.79$.

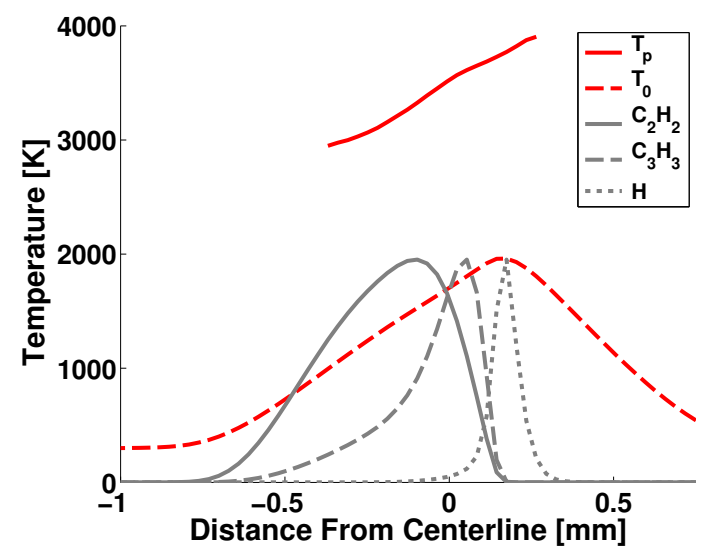

(c) $14 \mathrm{~atm}, a_{l}=135 \mathrm{~s}^{-1}, X_{C_{2} H_{4}}=0.41+$ (d) $14 \mathrm{~atm}, a_{l}=190 \mathrm{~s}^{-1}, X_{C_{2} H_{4}}=0.41+$ $X_{\mathrm{He}}=0.59, X_{\mathrm{O}_{2}}=0.1723+X_{\mathrm{He}}=$ 0.8277 . $X_{\mathrm{He}}=0.59, X_{\mathrm{O}_{2}}=0.1723+X_{\mathrm{He}}=$ 0.8277 .

Figure 4.5: Temperature profiles for several local strain rates at $4.065 \mathrm{~atm}$ and $14 \mathrm{~atm}$. Red line: LII measured peak particle temperature. Dashed red line: gas temperature. Gray lines: Normalized soot precursors $\mathrm{C}_{2} \mathrm{H}_{2}, \mathrm{C}_{3} \mathrm{H}_{3}$, and $\mathrm{H}$.

$\mathrm{C}_{2} \mathrm{H}_{2}, \mathrm{C}_{3} \mathrm{H}_{3}$, and $\mathrm{H}$ are shown to illustrate the expected sooting region on the fuel rich side of the counterflow flame configuration. Peak particle temperatures are maintained below the sublimation threshold around $4000 \mathrm{~K}$ for all cases presented. The peak temperature profiles are well described and follow the same general trend as the gradient in the local gas temperature. $\mathrm{C}_{2}$ laser induced fluorescence (LIF) interference is considered unlikely given the smooth peak particle temperature profiles and the use of a low peak laser fluence no greater than $0.11 \mathrm{~J} / \mathrm{cm}^{2}$ at $532 \mathrm{~nm}$. Spectroscopic tests for interference by Michelsen et al. [134] using a co-flow diffusion burner and Hofmann et al. [135] using a premixed flat 
flame burner at fluences of 0.15 and $0.115 \mathrm{~J} / \mathrm{cm}^{2}$ at $532 \mathrm{~nm}$ respectively exhibited no LIF interference. The reaction layer thickness defined by the width of the temperature profile scales as a function of $[28,136]$

$$
\delta / \delta_{0}=\sqrt{\frac{p_{0}}{p} \cdot \frac{a_{g, 0}}{a_{g}}} .
$$

Where $\delta$ is the reaction layer thickness and $a_{g}$ the global strain rate $a_{g}=4 v_{o x} / L$ where $v_{o x}$ is the oxidizer stream average velocity. The ambient pressure is represented by $p$. The 0 subscript represents a reference state. With a change in diffusion species from nitrogen to helium, the change in thermal diffusion must be taken into account and the more general expression holds for the reaction layer thickness using the mixture thermal diffusivity $\alpha$ $[28,136]$.

$$
\delta / \delta_{0}=\sqrt{\frac{\alpha}{\alpha_{0}} \cdot \frac{a_{g, 0}}{a_{g}}} .
$$

\subsection{Soot Particle Number Density}

Organizing the soot life cycle chronologically, this study begins with soot inception tied to the number density of nascent soot formed in the counterflow field. The counterflow configuration is ideal for studying and targeting the effects of soot inception due to its unique flame structure. Figure 4.6 shows several representative samples of normalized soot number density profiles (solid black line) along the counterflow burner axis of symmetry calculated from volume fraction and primary particle measurements using the relation $f_{v}=$ $\frac{\pi}{6} N d_{p}^{3}$. The same plot dimensions and definitions apply as in Fig. 4.5 above. The point of maximum flame temperature is represented by the vertical dashed red line. Gas velocity is calculated numerically and represented as the solid blue line with gas stagnation the vertical dashed blue line. The solid green line represents particle velocity in the flow with particle stagnation given by the vertical dashed green line. Molar species concentration profiles of $\mathrm{C}_{2} \mathrm{H}_{2}, \mathrm{C}_{3} \mathrm{H}_{3}$, and $\mathrm{H}$ are numerically calculated and normalized representing key 
precursor and soot growth species expected in the sooting region on the fuel rich side of the counterflow flame configuration. Particle velocity differs from the gas velocity due to

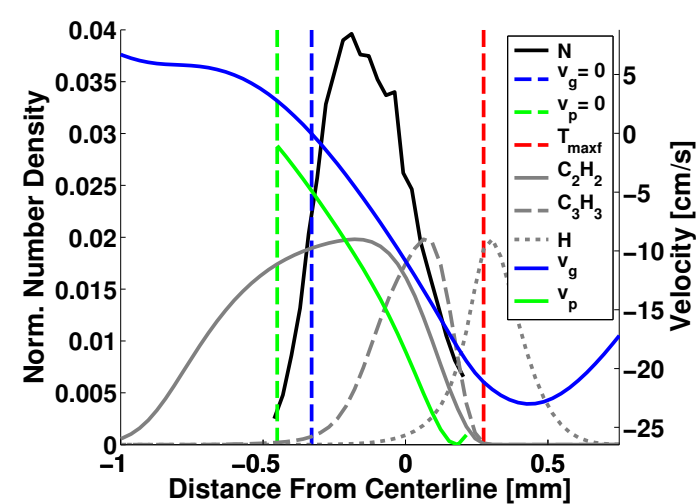

(a) $4.065 \mathrm{~atm}, a_{l}=119 \mathrm{~s}^{-1}, X_{C_{2} H_{4}}=0.5+$ $X_{N_{2}}=0.5, X_{O_{2}}=0.21+X_{N_{2}}=0.79$.

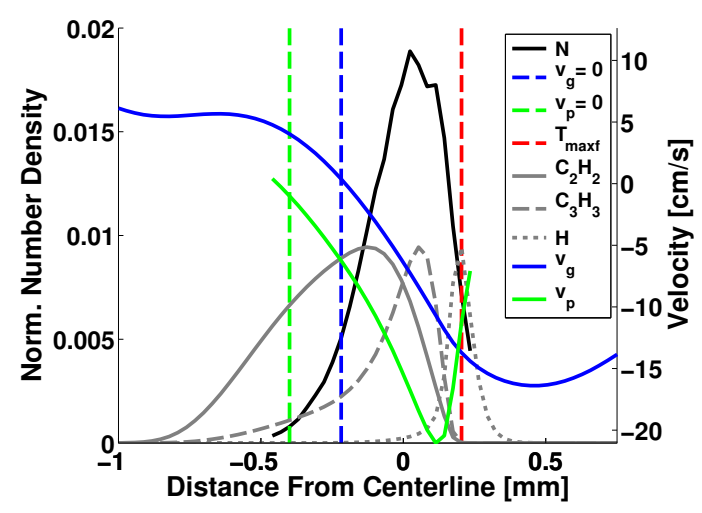

(c) $14 \mathrm{~atm}, a_{l}=135 \mathrm{~s}^{-1}, X_{C_{2} H_{4}}=0.41+$ $X_{\mathrm{He}}=0.59, X_{\mathrm{O}_{2}}=0.1723+X_{\mathrm{He}}=$ 0.8277 .

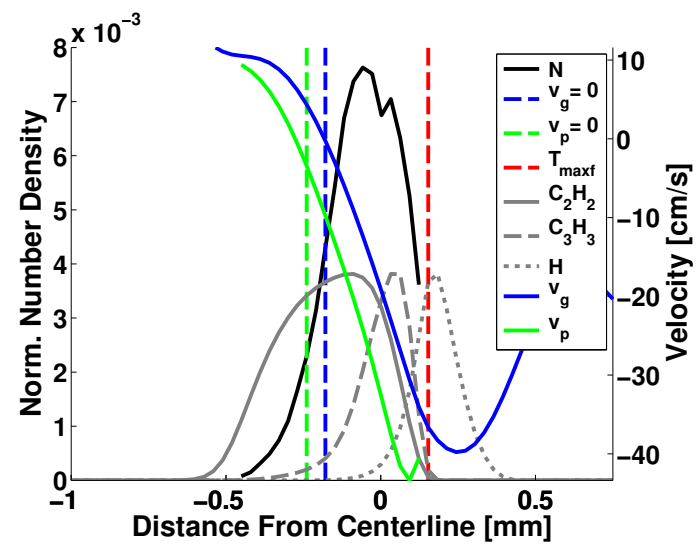

(b) $4.065 \mathrm{~atm}, a_{l}=408 \mathrm{~s}^{-1}, X_{C_{2} H_{4}}=0.5+$ $X_{N_{2}}=0.5, X_{O_{2}}=0.21+X_{N_{2}}=0.79$.

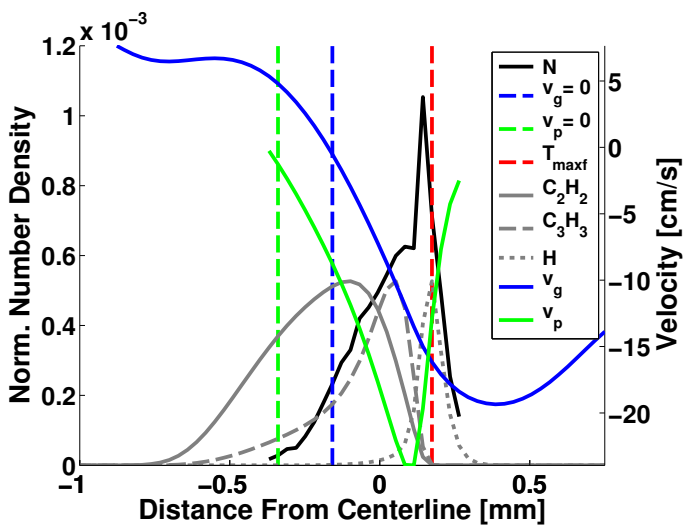

(d) $14 \mathrm{~atm}, a_{l}=190 \mathrm{~s}^{-1}, X_{C_{2} H_{4}}=0.41+$ $X_{\mathrm{He}}=0.59, X_{\mathrm{O}_{2}}=0.1723+X_{\mathrm{He}}=$ 0.8277 .

Figure 4.6: Normalized soot particle number density for several local strain rates at $4.065 \mathrm{~atm}$ and $14 \mathrm{~atm}$. Dashed red line: Point of maximum flame temperature. Gray lines: Normalized soot precursors $\mathrm{C}_{2} \mathrm{H}_{2}, \mathrm{C}_{3} \mathrm{H}_{3}$, and $\mathrm{H}$. Blue line: Local gas velocity. Dashed blue line: Gas stagnation. Green line: Local particle velocity. Dashed green line: Particle stagnation.

thermophoretic forces associated with the strong temperature gradient in the counterflow field effectively pushing the particle stagnation plane past gas stagnation in the negative $z$-direction. In order to analyze soot formation and growth, residence time must be well understood and therefore the thermophoretic force, $F_{T}$, should not be neglected in the counterflow field. Under the assumption of a continuum gas and particle to gas interaction 
in the transition regime, a model proposed by Talbot et al. [137],

$$
F_{T}=-\frac{12 \pi \mu \nu r_{p} C_{s}\left(\frac{\kappa}{\kappa_{s}}+C_{t} \frac{\lambda}{r_{p}}\right) \nabla T}{T\left(1+3 C_{m} \frac{\lambda}{r_{p}}\right)\left(1+2 \frac{\kappa}{\kappa_{s}}+2 C_{t} \frac{\lambda}{r_{p}}\right)},
$$

valid for all Knudsen numbers from free molecular to continuum was adopted here. The above formulation assumes a thermal accommodation coefficient $\alpha_{T}$ of unity where a contrary assumption is not expected to introduce significant errors according to analysis by Talbot et al. [137]. With the assumption of balance between thermophoretic force and Stokes drag force using the Millikian formula,

$$
F_{v}=-\frac{6 \pi \mu v_{T} r_{p}}{1+\frac{\lambda}{r_{p}}\left(A+B \exp ^{-C r_{p} / \lambda}\right)}
$$

the thermoporetic velocity, $v_{T}$ can be readily evaluated. Here, $\mu, \nu, \kappa, \lambda, T, \nabla T$ are the local gas mixture dynamic viscosity, the kinematic viscosity, thermal conductivity, mean free path, gas temperature, and gas temperature gradient respectively, while $r_{p}$ is the radius of the soot particle, and $\kappa_{s}$ the particle thermal conductivity. Optimum values for the thermal slip coefficient $C_{s}=1.17$, temperature jump coefficient $C_{t}=2.18$, and momentum exchange coefficient $C_{m}=1.14$ were determined from kinetic theory and provided in the work by Talbot et al. [137]. Coefficients A, B, and C in the Millikan drag formula were given as $1.2,0.41$, and 0.88 , respectively. The mean free path of the local gas mixture $\lambda$ is defined as,

$$
\lambda=\frac{\mu}{\rho} \sqrt{\frac{\pi W_{g}}{2 R T}} .
$$

The gas mixture viscosity can be obtained from numerical simulation or the simplified model using thermal conductivity for polyatomic gases and the Eucken formula for $f$ given by,

$$
\mu=\frac{\kappa W_{g}}{f R}(\gamma-1)
$$




$$
f=\frac{9 \gamma-5}{4}
$$

Utilizing the relation $\rho=p_{0} W_{g} / R T$ where $\rho$ is the gas density and $R$ the universal gas constant, the force balance between Eqs. 4.5 and 4.6 are solved for the thermophoretic velocity $v_{T}$

$$
v_{T}=-\frac{2 \kappa(\gamma-1) C_{s}\left(\frac{\kappa}{\kappa_{s}}+C_{t} \frac{\lambda}{r_{p}}\right)\left[1+\frac{\lambda}{r_{p}}\left(A+B \exp ^{-C r_{p} / \lambda}\right)\right] \nabla T}{f p_{0}\left(1+3 C_{m} \frac{\lambda}{r_{p}}\right)\left(1+2 \frac{\kappa}{\kappa_{s}}+2 C_{t} \frac{\lambda}{r_{p}}\right)},
$$

as a function of gas mixture thermal conductivity, heat capacity ratio $\gamma$, and ambient pressure $p_{0}$. The total particle velocity is a summation of the thermophoretic velocity and local gas velocity. The results of this formulation are in acceptable agreement with experiments in literature $[137,138]$. Uncertainties in the thermophoretic force mostly result from uncertainty in the thermal conductivity of soot. An average thermal conductivity of $1.4 \cdot 10^{-3}$ W/cmK was applied in this work without precise knowledge of the true value [139]. Uncertainty in the thermal conductivity results in up to a $20 \%$ change in the location of the particle stagnation plane. Additional model uncertainties inherent in the numerical simulations for gas velocity may be significant especially at high pressure. However, the magnitudes of model uncertainty are unknown and were not considered here.

It is useful to keep in mind that uncertainty calculated for number density is $91 \%$ for the nitrogen diluted cases at 4.065 atm and $82 \%$ for the helium diluted cases at 14 atm due to propagation of uncertainty from $d_{p}$ and $f_{v}$ using the relation between the three variables. In general however, uncertainties at one pressure taken over a range of strain rates and the same fuel and oxidizer concentrations should exhibit nearly the same uncertainty biases since the majority of variable uncertainties are shared over one experimental run at a given pressure. Additionally, the number density profiles change by around an order of magnitude or more over a range of strain rates making the trends in number density still useful quantities to analyze. The patterns inferred from the number density profiles in Fig. 
4.6 are clear whereby particles are nucleated at high temperature and maximum soot precursor concentration in the fuel rich region and reach a peak in number density early in terms of soot particle residence time in the flow. Peak number density is reached within the first $4 \mathrm{~ms}$ or less where total particle residence time is generally $10-20 \mathrm{~ms}$ in the counterflow flame. The early peak in number density is consistent with maximum nucleation rates at high temperature and maximum soot precursor concentration. Unlike premixed flames where soot nucleation and precursor formation rates must overcome oxidation rates at high temperature, the low concentration of oxidizing species in the sooting region of counterflow diffusion flames allows increasing temperature to predominantly increase fuel pyrolysis reactions and thereby increase precursor and soot nucleation rates [29]. Early onset of maximum soot number density is also consistent with soot formation times on the order of a few milliseconds [6] as opposed to 10-20 ms of total soot particle residence time in a typical counterflow flame flow field. Number Density then rapidly drops in the negative $z$-direction primarily due to a loss of temperature to sustain nucleation, primary particle coagulation, and the divergence in the counterflow field introducing a sink where particles are transported out of the measured centerline of interest.

The number density of soot nucleated early in the counterflow flame is the key to understanding soot processes further downstream. If a global reaction mechanism is considered, it is theorized that the maximum number density of soot is proportional to a measure of the global reaction rate $X_{c} e^{-E_{a} / R T_{\max }}$ where $X_{c}$ represents a molar concentration of some unknown species controlling soot nucleation, $E_{a}$ the activation energy of the global reaction, $R$ the universal gas constant, and $T_{\max }$ the maximum flame temperature. In Fig. 4.7, normalized maximum number densities of soot are plotted as a function of $1 / R T$ for pressures from 1 to $30 \mathrm{~atm}$ and a range of local strain rates. The exponential dependency of maximum number density on maximum flame temperature is evident for all cases. Along with a temperature dependency, nucleation of soot is believed to be second order in PAH concentration [6]. The use of a skeletal mechanism in modeling the flame structure and 


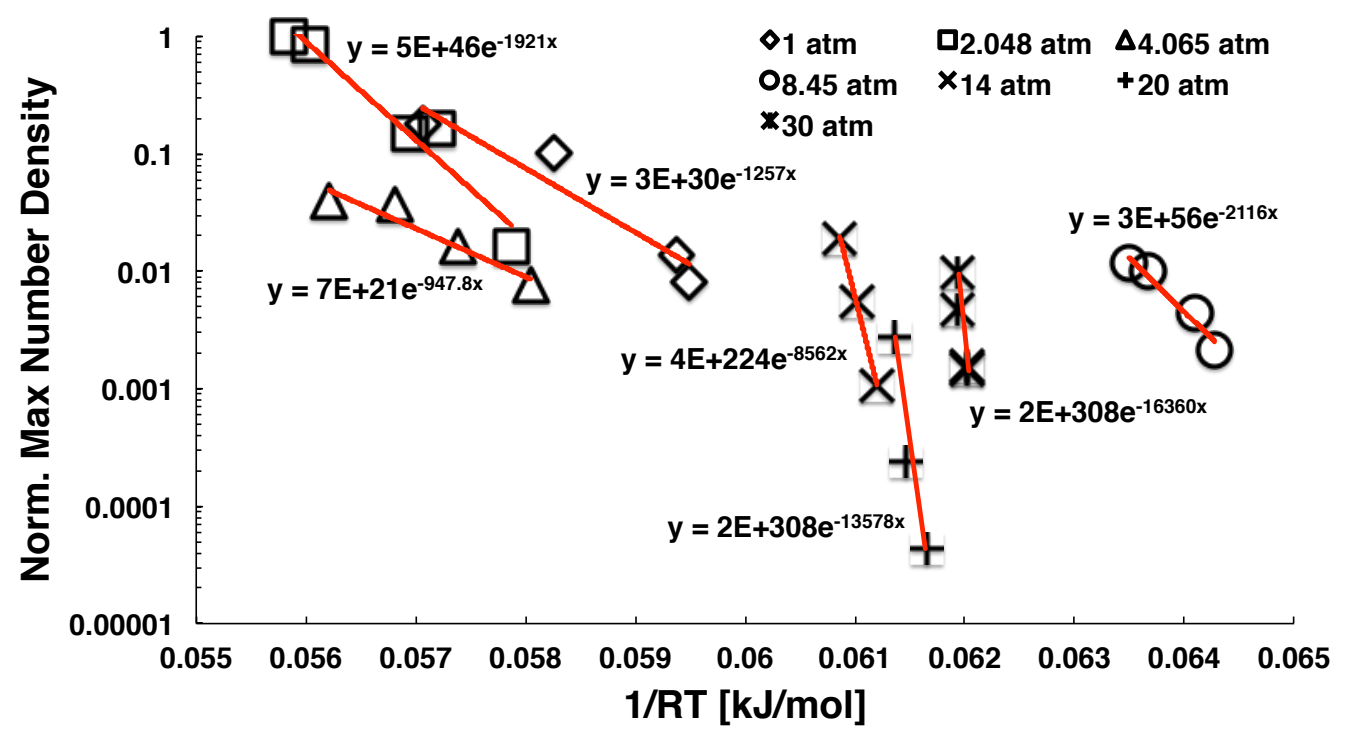

Figure 4.7: LII measured normalized maximum soot particle number density as a function of 1/RT over a range of pressures from 1 to $30 \mathrm{~atm}$ and a range of strain rates. Diamonds: $1 \mathrm{~atm}, a_{l}=91,202,335,367 \mathrm{~s}^{-1}, X_{C_{2} H_{4}}=1, X_{O_{2}}=0.21+X_{N_{2}}=0.79$. Squares: $2.048 \mathrm{~atm}, a_{l}=188,210,364,424,587 \mathrm{~s}^{-1}, X_{C_{2} H_{4}}=1, X_{O_{2}}=0.21+X_{N_{2}}=0.79$. Triangles: $4.065 \mathrm{~atm}, a_{l}=119,195,289,408 \mathrm{~s}^{-1}, X_{C_{2} H_{4}}=0.5+X_{N_{2}}=0.5, X_{O_{2}}=$ $0.21+X_{N_{2}}=0.79$. Circles: $8.45 \mathrm{~atm}, a_{l}=104,119,163,186 \mathrm{~s}^{-1}, X_{C_{2} H_{4}}=0.4+X_{N_{2}}=$ 0.6, $X_{O_{2}}=0.1681+X_{N_{2}}=0.8319 . \mathrm{X}: 14 \mathrm{~atm}, a_{l}=135,158,190 \mathrm{~s}^{-1}, X_{C_{2} H_{4}}=0.41+$ $X_{H e}=0.59, X_{O_{2}}=0.1723+X_{H e}=0.8277$. Cross: $20 \mathrm{~atm}, a_{l}=110,128,160 \mathrm{~s}^{-1}$, $X_{C_{2} H_{4}}=0.4+X_{H e}=0.6, X_{O_{2}}=0.1681+X_{H e}=0.8319$. Double Cross: $30 \mathrm{~atm}$, $a_{l}=88,100 \mathrm{~s}^{-1}, X_{C_{2} H_{4}}=0.39+X_{H e}=0.61, X_{O_{2}}=0.1639+X_{H e}=0.8361$.

species concentrations did not allow for higher order PAH species to be analyzed. The accuracy in predicting molar concentrations of any higher order species is also questionable. $\mathrm{C}_{2} \mathrm{H}_{2}$ is theorized to play a significant role in PAH formation and soot nucleation $[6,133]$ and its relative abundance in the flame region make it a useful potential marker to explore. The effect of $\mathrm{C}_{2} \mathrm{H}_{2}$ concentration, diffusive and convective fuel flux, and total fuel density was explored as a marker for soot nucleation. No consistent reaction rate dependency was observed for any of the target species. An analysis of the slopes in Fig 4.7 illustrate considerable variation between different pressures and fuel-oxidizer-diluent concentrations. Taking the data from 1 to $8.45 \mathrm{~atm}$, all flames are diluted with nitrogen. We can extract the effective activation energy of the global reaction from the slopes of the curves of each sample in Fig. 4.7. For nitrogen diluted flames at pressures from 1 to $8.45 \mathrm{~atm}$, the activation 
energy is in the range of 1000-2000 kJ/mol. A marked difference is evident when analyzing the data from 14 to 30 atm diluted with helium. The activation energy increases by an order of magnitude to $9000-16000 \mathrm{~kJ} / \mathrm{mol}$ for the high pressure helium diluted samples. Comparing all activation energies suggests that activation energy increases with increasing pressure and a change in diluent from nitrogen to helium. Increasing activation energy suggests a greatly increased sensitivity on temperature and difficulty in nucleating soot. The analysis is complicated however, as many competing effects occur at once including effects due to changes in pressure, gas composition, fuel or soot precursor species concentrations, flame structure changes, and changes in residence time.

Figure 4.6 is useful for illustrating the spatial changes in soot number density in the counterflow flame. Yet the rate chemistry driving soot formation and particle surface growth are better understood from a temporal perspective. Normalized soot number density profiles (black lines) along the burner axis of symmetry as a function of soot particle residence time are given in Fig. 4.8. Cases at a pressure of $4.065 \mathrm{~atm}$ and local strain rates of $a_{l}=119,195,289$, and $408 \mathrm{~s}^{-1}$ are shown in Fig 4.8 a) and b). Datasets at a pressure of $14 \mathrm{~atm}$ and local strain rates of $a_{l}=135,158$, and $190 \mathrm{~s}^{-1}$ are given in c) and d). Fig. 4.8 a) and c) illustrate the local gas temperature variation (red lines) as a function of particle residence time for the stated local strain rate cases along with the variation in soot number density. Fig. 4.8 b) and d) indicate acetylene molar concentration (gray lines) as a function of particle residence time along with soot number density. The point of maximum flame temperature was employed as the $t=0$ point of soot nucleation for comparison between different strain rates. Particle residence time was calculated by $t=\sum \Delta x / v(x)$ using the trapezoidal rule. The total particle velocity was employed taking into account the thermophoretic force acting on the soot particles in the presence of the steep flame temperature gradient. As in the spatial representation of number density in Fig. 4.6, the right hand side of the figure corresponding to $t=0$ is on the oxidizer side at the point of maximum flame temperature. The left side corresponds to the fuel rich region. When analyzing all given 
cases, it is evident that the maximum number density shifts to shorter residence time with increasing strain rate and decreases in magnitude. Temperature was previously identified as the most dominant mechanism controlling soot nucleation in a global sense. The effect of changes in local temperature on maximum soot number density are most obvious in Fig. 4.8 a) for the nitrogen diluted $4.065 \mathrm{~atm}$ samples. Increased flame strain marked by increasing local strain rate reduces reaction time and thus maximum flame temperature. The temperature profile also drops rapidly towards shorter residence time with increasing strain rate. The loss of high temperature earlier in the flow field at higher strain rates effectively stifles further nucleation of soot. Thus the maximum soot particle number density is manifested more rapidly and is halted more rapidly at higher strain rates. The change in temperature is not as obvious for the $14 \mathrm{~atm}$ case shown in Fig. $4.8 \mathrm{c}$ ). However, the effect of temperature on soot nucleation is much more pronounced at 14 atm with helium dilution and is manifested by a greater global activation energy determined previously from Fig. 4.7. The temporal shift in number density profiles may also be partially explained by the very similar shift in the peak acetylene molar concentration towards shorter residence time. Acetylene molar concentration is a useful marker and widely considered governing species for soot nucleation. Other contributors including PAH species and radicals are also considered to play a role [6]. PAH species profiles likely exhibit the same temporal shift towards shorter residence time due to higher local velocities from increased flame strain and higher global flow velocity. The temporal shift of $\mathrm{C}_{2} \mathrm{H}_{2}$ molar concentration and number density is most evident for the $4.065 \mathrm{~atm}$ case given in Fig. $4.8 \mathrm{~b}$ ) but is still observed for the 14 atm case in $d$ ), the difference being a greater magnitude change in local strain rates for the 4.065 atm case. Changes in flame structure from the nitrogen diluted 4.065 atm flame to the 14 atm helium diluted flame may also play a role manifested primarily by changes in species diffusion rates.

It is useful to recall that the maximum soot particle number density is achieved at the point where the soot particle nucleation and particle transport rates are overtaken by rates 
of coagulation and mass loss from flow divergence. This maximum soot number density occurs around $3 \mathrm{~ms}$ at $4.065 \mathrm{~atm}, a_{l}=119 \mathrm{~s}^{-1}$ and decreases in time with higher strain rate to approximately $1 \mathrm{~ms}$ with pressure constant for $a_{l}=408 \mathrm{~s}^{-1}$ due primarily to an earlier cut off in the nucleation rate from temperature and species effects. Peak number density occurs around $1.5 \mathrm{~ms}$ at $14 \mathrm{~atm}, a_{l}=135 \mathrm{~s}^{-1}$ and decreases to fractions of a millisecond at $14 \mathrm{~atm}, a_{l}=190 \mathrm{~s}^{-1}$. While the effect of temperature is most evident globally, the controlling mechanisms for soot number density are clearly a coupled species and temperature effect and their magnitudes on the rate of soot nucleation competing against rates of particle coagulation and mass loss in residence time space.
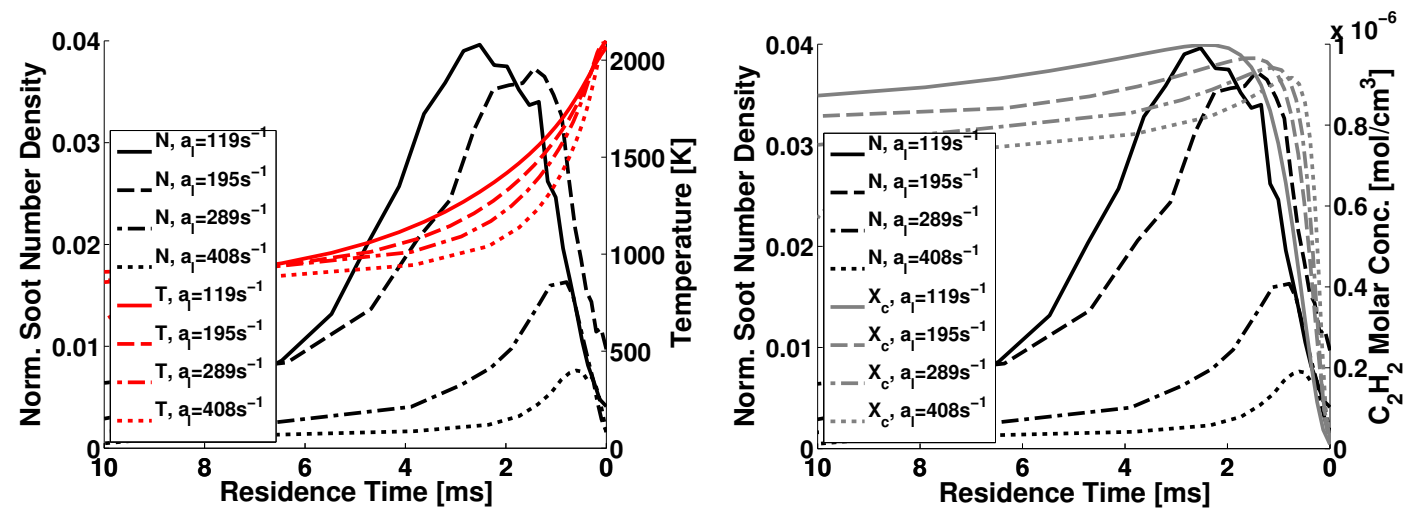

(a) $4.065 \mathrm{~atm}, X_{C_{2} H_{4}}=0.5+X_{N_{2}}=0.5$, $X_{\mathrm{O}_{2}}=0.21+X_{N_{2}}=0.79$.

(b) $4.065 \mathrm{~atm}, X_{C_{2} H_{4}}=0.5+X_{N_{2}}=0.5$, $X_{O_{2}}=0.21+X_{N_{2}}=0.79$.

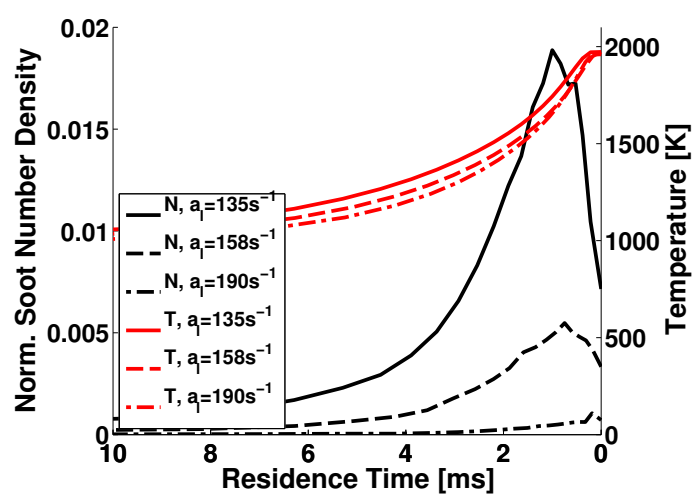

(c) $14 \mathrm{~atm}, X_{C_{2} H_{4}}=0.41+X_{H e}=0.59$, $X_{O_{2}}=0.1723+X_{H e}=0.8277$.

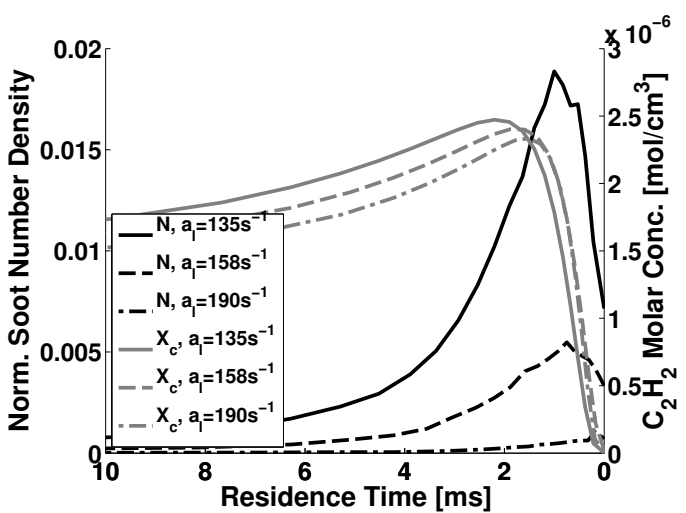

(d) $14 \mathrm{~atm}, X_{C_{2} H_{4}}=0.41+X_{H e}=0.59$, $X_{\mathrm{O}_{2}}=0.1723+X_{\mathrm{He}}=0.8277$.

Figure 4.8: Normalized soot particle number density as a function of particle residence time for several local strain rates at $4.065 \mathrm{~atm}$ and $14 \mathrm{~atm}$. Red lines: Local gas temperature. Gray lines: $\mathrm{C}_{2} \mathrm{H}_{2}$ molar concentration. 
Analyzing the rate of soot particle nucleation provides further insight into the chemical kinetics at play in the soot layer. Figure 4.9 shows the normalized soot number density growth rate (black lines) as a function of particle residence time in the soot layer. Temperature profiles (red lines) are also given for reference. Correcting for the rate of particle number density lost due to flow divergence is important. Assuming a monodisperse particle distribution i.e. equal mass of all particles, the mass loss rate due to flowfield divergence is proportional to the number density. The number density loss rate is calculated according to $d N / d t=2 \pi U(z) N$ where $U(z)$ is the radial velocity gradient parameter and $N$ the local number density of soot particles. By conservation of mass in the counterflow configuration the axial velocity gradient $d v_{z} / d z=-2 U$ and the radial velocity $v_{r}=r U(z)$. The rate of number density lost is added back to the number density growth rate to correct the total rate of particle nucleation produced in the flame. First considering the case of $4.065 \mathrm{~atm}$, $a_{l}=125 \mathrm{~s}^{-1}$ from Fig. 4.9 a), the soot nucleation growth rate increases from zero to a maximum at a particle residence time of $1.5 \mathrm{~ms}$. The rate decays back to zero within a particle residence time of $5 \mathrm{~ms}$. For $4.065 \mathrm{~atm}, a_{l}=435 \mathrm{~s}^{-1}$ the nucleation rate rate increases from zero to a maximum at a particle residence time of $0.1 \mathrm{~ms}$ and decays rapidly to zero within $1.5 \mathrm{~ms}$. The increased time for nucleation growth with decreasing strain rate is primarily an effect of additional residence time at higher temperature and higher species concentrations. The coagulation rate at $4.065 \mathrm{~atm}$ is likely too small to have an effect on the nucleation rate. The maximum loss in number density due to coagulation at $4.065 \mathrm{~atm}, a_{l}=125 \mathrm{~s}^{-1}$ is nearly five orders of magnitude lower than the maximum nucleation rate. Coagulation rates were calculated using Fuchs model for particle coagulation in the transition regime from Kazakov and Frenklach [140]. Assumptions include a monodisperse particle diameter distribution equal to local LII measured particle diameter, a soot density of $1.8 \mathrm{~g} / \mathrm{cm}^{3}$, and local gas properties provided by numerical simulation. Perturbing assumptions by estimated uncertainties does not significantly affect the resulting collision rate. Rates of small soot particles colliding with larger particles are higher and may play a greater role than 


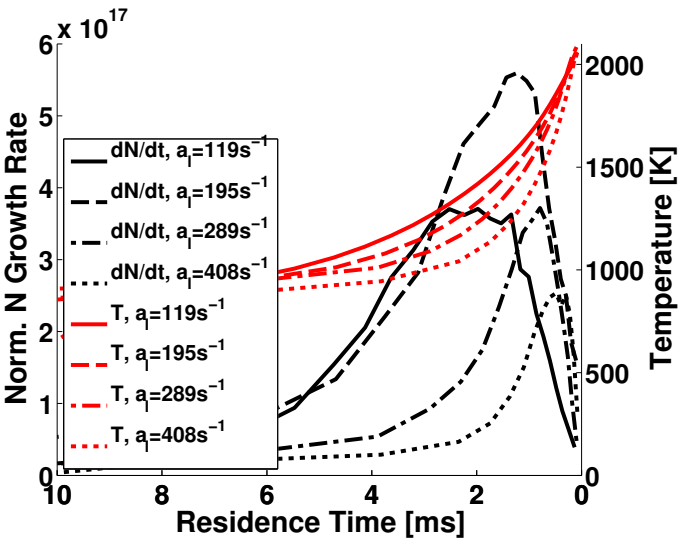

(a) $4.065 \mathrm{~atm}, X_{C_{2} H_{4}}=0.5+X_{N_{2}}=0.5$, $X_{O_{2}}=0.21+X_{N_{2}}=0.79$.

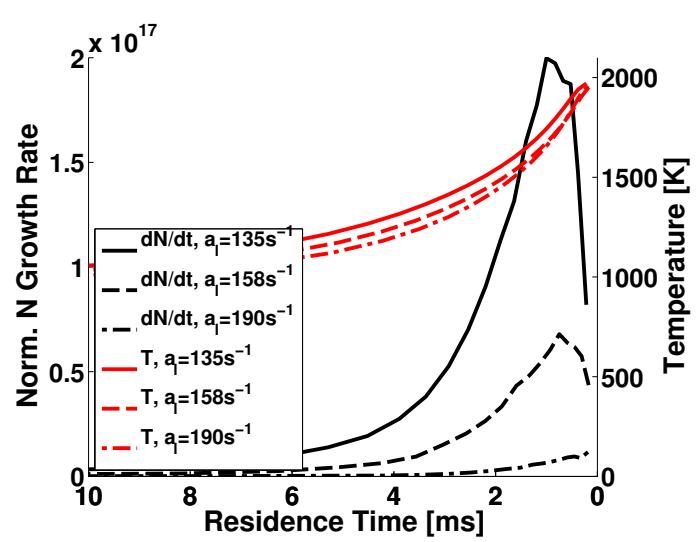

(b) $14 \mathrm{~atm}, X_{\mathrm{C}_{2} \mathrm{H}_{4}}=0.41+X_{\mathrm{He}}=0.59$, $X_{\mathrm{O}_{2}}=0.1723+X_{\mathrm{He}}=0.8277$.

Figure 4.9: Normalized soot particle number density growth rates as a function of particle residence time for several local strain rates at $4.065 \mathrm{~atm}$ and $14 \mathrm{~atm}$. Red lines: Local gas temperature.

monodisperse coagulation. However, the effect is not accurately quantifiable without detailed soot modeling or experimental knowledge of the local particle distribution. For the case of $14 \mathrm{~atm}, a_{l}=135 \mathrm{~s}^{-1}$ in Fig. $4.9 \mathrm{~b}$ ), the nucleation growth rate increases to a maximum at a particle residence time of $0.4 \mathrm{~ms}$ and decays to zero within $3 \mathrm{~ms}$. Lastly, for the case of $14 \mathrm{~atm}, a_{l}=190 \mathrm{~s}^{-1}$ the number density growth rate increases to a maximum at $0.2 \mathrm{~ms}$ of particle residence time and decays to zero within $1.5 \mathrm{~ms}$. Number density loss rates due to particle coagulation are still insignificant at $14 \mathrm{~atm}$.

Assuming that growth in number density can only be due to nucleation, a lower temperature limit on the nucleation growth rate mechanism can be identified for the 4.065 atm and 14 atm cases by analyzing the time at which the rate of number density growth is halted. For the $4.065 \mathrm{~atm}$ cases presented, decay times of $5 \mathrm{~ms}$ for $a_{l}=125 \mathrm{~s}^{-1}$ and $1.5 \mathrm{~ms}$ for $a_{l}=435 \mathrm{~s}^{-1}$ result in a lower limit temperature range of $1040-1200 \mathrm{~K}$. For the cases analyzed at $14 \mathrm{~atm}$, decay times of $3 \mathrm{~ms}$ and $1.5 \mathrm{~ms}$ for local strain rates of $a_{l}=135 \mathrm{~s}^{-1}$ and $a_{l}=190 \mathrm{~s}^{-1}$ respectively results in a volumetric soot growth cutoff temperature range of $1400-1530 \mathrm{~K}$. Changes in soot precursor species concentrations clearly also play a role in the temperature range of the nucleation cutoff. However, significant $\mathrm{C}_{2} \mathrm{H}_{2}$ and likely 
other precursor species are still present in the region where nucleation is halted at $14 \mathrm{~atm}$ so the effect appears to be primarily due to temperature, pressure, and flame diluent and not precursor species. Several explanations for nucleation behavior can be developed after analyzing the full spectrum of data. Measurements at 1, 2.048, and 4.065 atm demonstrate a low cutoff temperature range around 1000-1200 K and the lowest global activation energies from Fig. 4.7 indicating a pressure and diluent effect on the chemical kinetics. This results in $1,2.048$, and 4.065 atm being the most conducive for soot nucleation. Conversely, the cutoff temperature range is several hundred degrees higher around $1400-1550 \mathrm{~K}$ and the global activation energy an order of magnitude higher at 14, 20, and 30 atm indicating a much greater barrier to soot nucleation and a significant change from low pressure nitrogen diluted flames. The nucleation cutoff temperature is consistent over all pressures analyzed using the same diluent species. This suggests the change in nucleation cutoff temperature is a diluent effect due to the necessity of changing from nitrogen to helium at higher pressure to maintain laminar flow. The observation also explains the order of magnitude change in global activation energy with a change to helium diluent as shown in Fig. 4.7. However, a consistent decrease in nucleation rates with increasing pressure while accounting for changes in temperature still indicate a pressure effect on the chemical kinetics. Additional evidence can be disseminated by analyzing global activation energies presented in Fig. 4.7 which increase with increasing pressure with diluent held fixed. Perturbing the results by more than measurement uncertainty does not change the consistent trend. Detailed causes for the effects of pressure and diluent on the chemical kinetics of soot nucleation are beyond the scope of the global perspective analyzed here.

The full mechanism for soot nucleation is largely speculative in literature. It is generally accepted that some form of hydrogen abstraction $\mathrm{C}_{2} \mathrm{H}_{2}$ addition (HACA) and propargyl recombination chemical mechanisms play a role in forming soot precursors at high temperature. Among the proposed chemical mechanisms, other pathways including a physical PAH coagulation and condensation process have been proposed especially applicable at 
lower temperatures $[6,29,48,133,141,142]$. The low inception cutoff temperature range of $1040-1200 \mathrm{~K}$ at $4.065 \mathrm{~atm}$ suggests both a high temperature chemical pathway and a low temperature PAH coagulation pathway is active. Calculations reported by Totton et al. [143] indicate at temperatures of 1000-1300 K hexabenzocoronene and circumcoronene are the most likely candidates for physical dimerization initiating low temperature soot nucleation. Conversely at $14 \mathrm{~atm}$, the high cutoff temperature range of $1400-1530 \mathrm{~K}$ indicates a high temperature chemical pathway may be dominant with a low temperature PAH coagulation mechanism stifled. However, these explanations are largely speculative due to the highly uncertain nature of the nucleation mechanism.

It is important to examine the uncertainty associated with the estimated rates of nucleation. Uncertainties result from the number density measurement, the thermal conductivity of soot dictating the thermophoretic force and soot particle residence time, and particle distribution assumption. Number density measurement uncertainty is approximately $82 \%$ for helium-diluted flames and $91 \%$ for nitrogen-diluted flames. Assuming a monodisperse particle diameter distribution when calculating the number density of the ensemble intro-

duces an error of approximately 15\%. Assuming a lognormal distribution with $\sigma_{d_{p, g}}=1.19$ removes most of the error owing to the mean of both distributions being nearly identical as solutions to the LII model. Residence time uncertainty is estimated as approximately $15 \%$ not accounting for unknown uncertainties in gas velocity from numerical modeling. Measurement uncertainty is dominant resulting in total uncertainties of $85 \%$ for heliumdiluted flames and $93 \%$ for nitrogen-diluted flames. Conservative practice would dictate that uncertainty around one order of magnitude is advisable.

\subsection{Soot Volume Fraction}

Many authors have studied the pressure effect on total volume fraction of soot produced in flames using various burner configurations. Diverse proportionalities of soot loading with 
pressure applicable to different pressure ranges were reported for a range of burners and fuels $[9,29]$. This study instead attempts to focus on underlying mechanisms. The growth of soot volume fraction and the growth of soot particle size are closely tied to the surface growth mechanisms present in the counterflow flame configuration. Figure 4.10 shows normalized soot volume fraction profiles along the burner axis of symmetry calculated from the peak soot incandescence signal at the measured peak temperature using LII. The same plot dimensions and definitions apply as in Fig. 4.6 above. A volume of soot in the flame initiates during particle nucleation near the maximum flame temperature. The nascent soot particles are convected through the flow in the negative $z$-direction due to Stokes drag and thermophoresis from the steep temperature gradient in the sooting region. As the particles traverse this region soot surface growth mechanisms and new particle nucleation increase the local soot volume fraction while the divergence in the counterflow field simultaneously introduces a sink, removing soot mass (or volume) from the measurement plane. Particle coagulation has no effect on soot volume fraction from a volume conservation perspective. Soot volume fraction reaches a peak when the loss of mass due to flow divergence is equal to the new rates of mass growth on the particle surface and particle nucleation. The peak is observed near the gas stagnation plane where maximum flow divergence is achieved. The local volume fraction of soot drops rapidly after the peak due to strong flow divergence and steeply decreasing local temperature staunching further surface growth and nucleation. Comparing the reaction layer thickness defined by the thickness of the temperature profile to the soot layer thickness for consistency results in an interesting observation. Reaction layer thickness and soot layer thickness are proportional for all samples at pressures between $1 \mathrm{~atm}$ and $14 \mathrm{~atm}$. The relationship begins to diverge at $20 \mathrm{~atm}$ and significantly changes for $30 \mathrm{~atm}$ samples where the soot layer thickness increases in a nonlinear manner compared to reaction layer thickness. The reason for this effect is unknown. The effect is not believed to be due to a turbulent transition. All data was collected below a previously identified turbulent Reynolds number limit of approximately 2600 and no significant turbu- 
lent breakup of the soot layer was observed. In all cases the soot volume fraction profiles are well formed and are associated with the lowest measurement uncertainty of all LII derived variables at $30 \%$. Order of magnitude changes in soot loading can be explored with the counterflow burner providing valuable data trends.

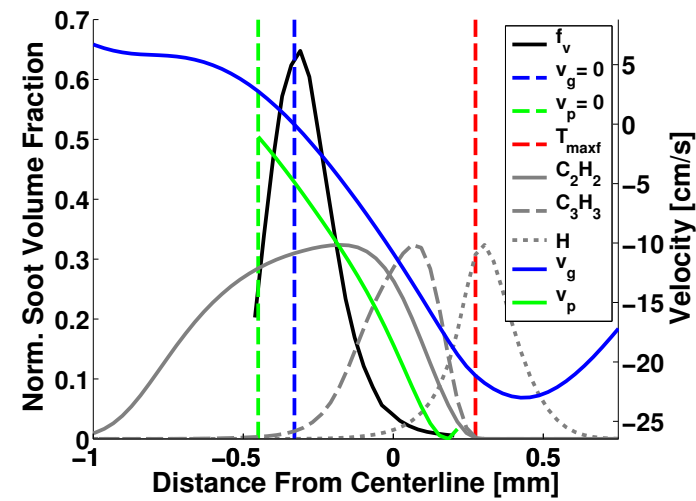

(a) $4.065 \mathrm{~atm}, a_{l}=119 \mathrm{~s}^{-1}, X_{C_{2} H_{4}}=0.5+$ $X_{N_{2}}=0.5, X_{O_{2}}=0.21+X_{N_{2}}=0.79$.

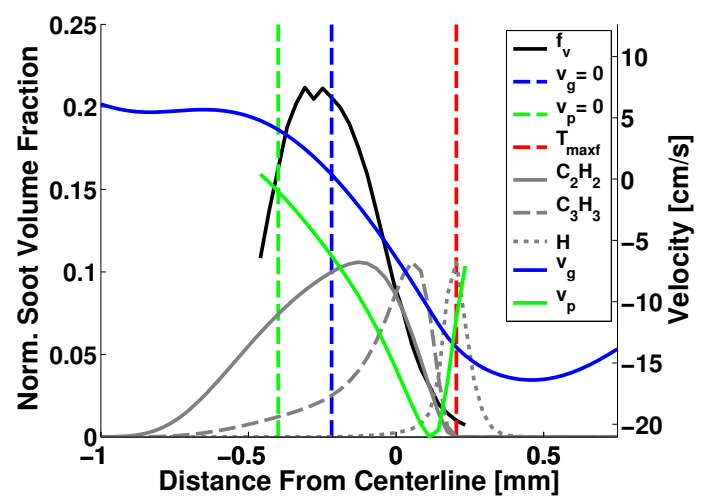

(c) $14 \mathrm{~atm}, a_{l}=135 \mathrm{~s}^{-1}, X_{C_{2} H_{4}}=0.41+$ $X_{\mathrm{He}}=0.59, X_{\mathrm{O}_{2}}=0.1723+X_{\mathrm{He}}=$ 0.8277 .

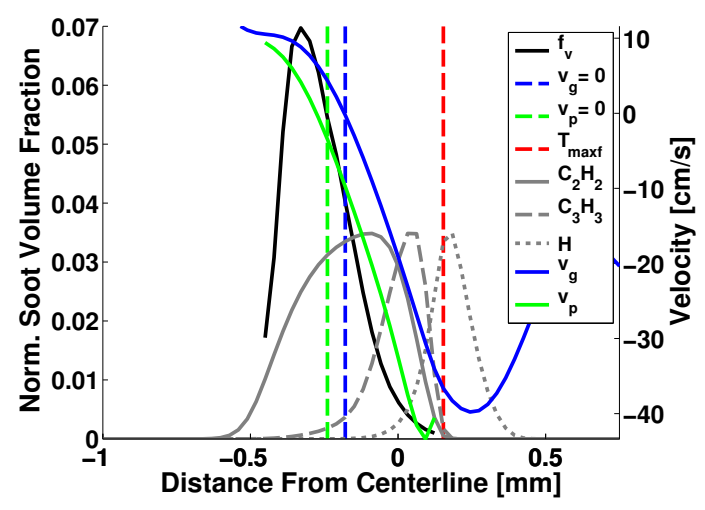

(b) $4.065 \mathrm{~atm}, a_{l}=408 \mathrm{~s}^{-1}, X_{C_{2} H_{4}}=0.5+$ $X_{N_{2}}=0.5, X_{O_{2}}=0.21+X_{N_{2}}=0.79$.

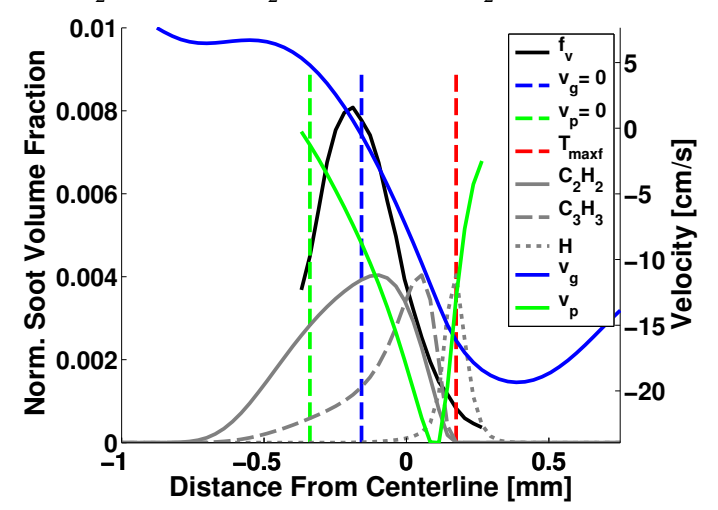

(d) $14 \mathrm{~atm}, a_{l}=190 \mathrm{~s}^{-1}, X_{C_{2} H_{4}}=0.41+$ $X_{\mathrm{He}}=0.59, X_{\mathrm{O}_{2}}=0.1723+X_{\mathrm{He}}=$ 0.8277 .

Figure 4.10: Normalized soot volume fraction for several local strain rates at $4.065 \mathrm{~atm}$ and $14 \mathrm{~atm}$. Dashed red line: Point of maximum flame temperature. Gray lines: Normalized soot precursors $\mathrm{C}_{2} \mathrm{H}_{2}, \mathrm{C}_{3} \mathrm{H}_{3}$, and $\mathrm{H}$. Blue line: Local gas velocity. Dashed blue line: Gas stagnation. Green line: Local particle velocity. Dashed green line: Particle stagnation.

Maintaining a global perspective, Fig. 4.11 demonstrates the relationship between normalized peak soot particle number density and normalized peak soot volume fraction produced in counterflow flames over a range of pressures, fuel-oxidizer concentrations, and strain rates. Linear trend lines are provided to guide the eye. A linear relationship be- 
tween maximum number density and maximum soot volume fraction was evident for all pressures over a range of local strain rates. This indicates that on a global scale the key factor controlling the volume fraction of soot produced in a counterflow flame is the initial maximum number density of soot particles first nucleated in the flame. Some consensus in literature ( [7] and citations within) has reached the same conclusion. Furthermore, the trend indicates that the mechanism controlling the particle surface growth rate is relatively insensitive to changes in local strain rate and that PAH species concentrations, particle residence time, and temperature must be similar in the particle growth region of the soot layer.

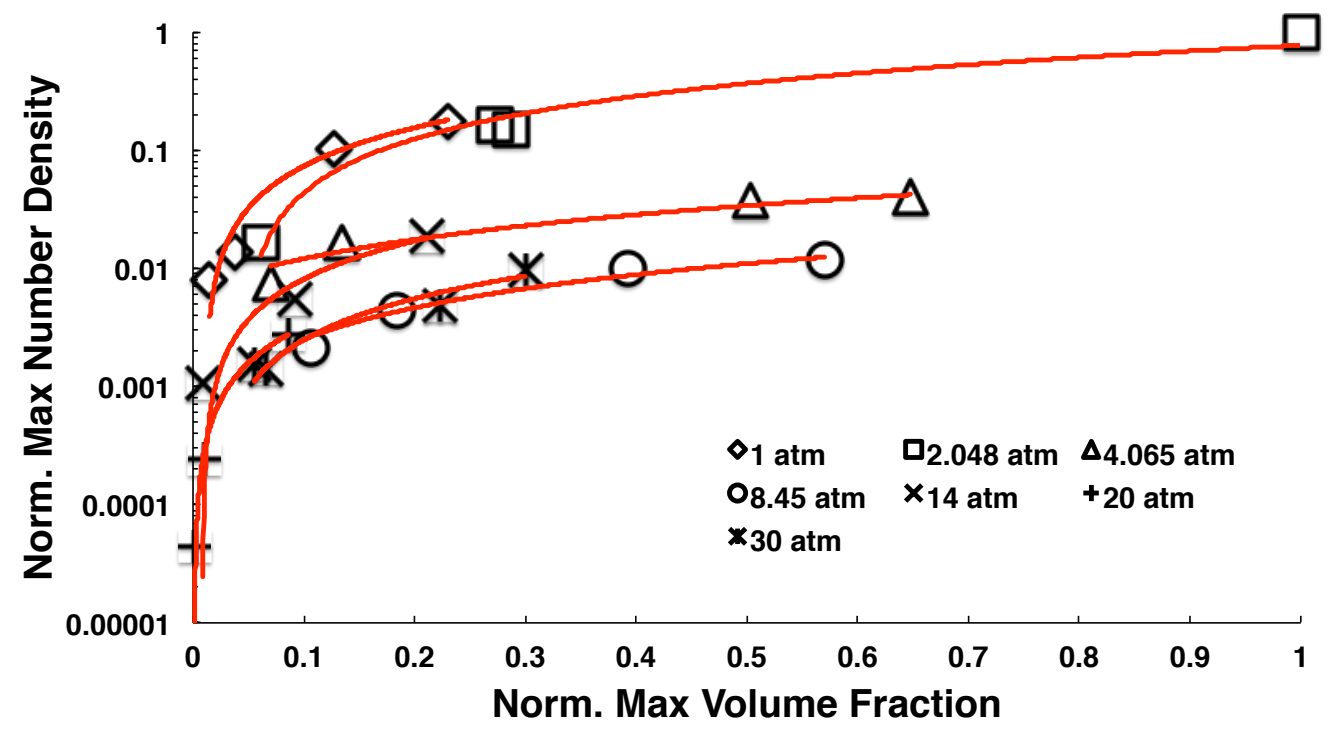

Figure 4.11: LII measured normalized maximum soot particle number density as a function of volume fraction over a range of pressures from 1 to $30 \mathrm{~atm}$ and a range of strain rates. Diamonds: $1 \mathrm{~atm}, a_{l}=91,202,335,367 \mathrm{~s}^{-1}, X_{C_{2} H_{4}}=1, X_{O_{2}}=0.21+X_{N_{2}}=0.79$. Squares: $2.048 \mathrm{~atm}, a_{l}=188,210,364,424,587 \mathrm{~s}^{-1}, X_{C_{2} H_{4}}=1, X_{O_{2}}=0.21+X_{N_{2}}=$ 0.79. Triangles: $4.065 \mathrm{~atm}, a_{l}=119,195,289,408 \mathrm{~s}^{-1}, X_{C_{2} H_{4}}=0.5+X_{N_{2}}=0.5$, $X_{O_{2}}=0.21+X_{N_{2}}=0.79$. Circles: $8.45 \mathrm{~atm}, a_{l}=104,119,163,186 \mathrm{~s}^{-1}, X_{C_{2} H_{4}}=0.4+$ $X_{N_{2}}=0.6, X_{O_{2}}=0.1681+X_{N_{2}}=0.8319 . \mathrm{X}: 14 \mathrm{~atm}, a_{l}=135,158,190 \mathrm{~s}^{-1}, X_{C_{2} H_{4}}=$ $0.41+X_{H e}=0.59, X_{O_{2}}=0.1723+X_{H e}=0.8277$. Cross: 20 atm, $a_{l}=110,128,160 \mathrm{~s}^{-1}$, $X_{C_{2} H_{4}}=0.4+X_{H e}=0.6, X_{O_{2}}=0.1681+X_{H e}=0.8319$. Double Cross: $30 \mathrm{~atm}$, $a_{l}=88,100 \mathrm{~s}^{-1}, X_{C_{2} H_{4}}=0.39+X_{H e}=0.61, X_{O_{2}}=0.1639+X_{H e}=0.8361$.

Seeking greater detail, the temporal evolution of soot volume fraction is given in Fig. 4.12 illustrating LII measured normalized soot volume fraction profiles (black lines) along 
the axial centerline of the counterflow burner as a function of particle residence time in the soot layer. Samples at the same two pressures of $4.065 \mathrm{~atm}$ and $14 \mathrm{~atm}$ are presented as above. At $4.065 \mathrm{~atm}$, soot volume fraction profiles for local strain rates of $a_{l}=119,195,289$, and $408 \mathrm{~s}^{-1}$ are given next to the corresponding local gas temperature profiles (red lines) in Fig. 4.12 a) and $\mathrm{C}_{2} \mathrm{H}_{2}$ concentration profiles (gray lines) in Fig $4.12 \mathrm{~b}$ ). At $14 \mathrm{~atm}$ soot volume fraction profiles for local strain rates of $a_{l}=135,158$, and $190 \mathrm{~s}^{-1}$ are given next to the corresponding local gas temperature profiles in Fig. 4.12 c) and $\mathrm{C}_{2} \mathrm{H}_{2}$ concentration profiles in d). Acetylene is believed to be the primary species involved in surface reactions $[6,133]$. The same orientation and characterization applies as was presented in Fig. 4.8 above. A clear trend of decreasing soot volume fraction with increasing strain rate is evident. The decrease in maximum soot volume fraction with increasing strain rate is due primarily to a decrease in maximum flame temperature, a decrease in residence time at high temperature, and a decrease in particle nucleation. Fig. 4.12 a) and c) illustrates that the temperature profiles decay earlier with increasing strain rate and correspond to a similar temporal change in the growth of soot volume fraction. Thus the driving mechanism of the shift to earlier residence time is likely a drop in temperature below a limit where rapid soot growth and nucleation are halted. Comparing Figs. 4.8 and 4.12 shows that maximum volume fraction and number density profiles exhibit similar proportionality indicating the number density of nascent soot plays a large role in determining maximum soot volume fraction later downstream. Residence time between maximum soot number density and maximum soot volume fraction remain approximately constant ( $2 \mathrm{~ms}$ for $4.065 \mathrm{~atm}, 3 \mathrm{~ms}$ for $14 \mathrm{~atm}$ ) only shifted to earlier time, indicating a constant growth period at similar temperatures consistent with the linear relationship between volume fraction and number density. The molar concentrations of $\mathrm{C}_{2} \mathrm{H}_{2}$ given in Fig. 4.12 b) and d) show a variation of only 15-20\% between the lowest and highest strain rate cases presented. The small variation is likely inadequate to cause large variations in nucleation and soot surface growth with changes in strain rate. A more detailed modeling effort may 
uncover finer changes due to temperature, species, and residence time than can be observed here. The evidence clearly suggests that maximum soot nucleation number density is the determinant of maximum soot volume fraction later in the soot layer with other effects including species, temperature, and residence time changes playing a lesser role.

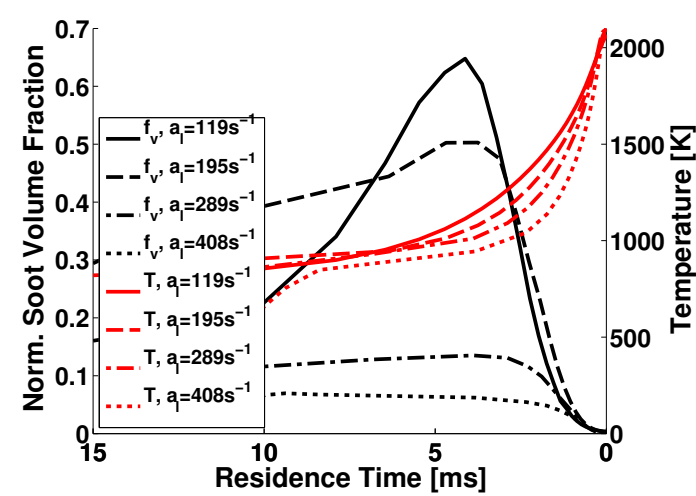

(a) $4.065 \mathrm{~atm}, X_{\mathrm{C}_{2} \mathrm{H}_{4}}=0.5+X_{N_{2}}=0.5$, $X_{O_{2}}=0.21+X_{N_{2}}=0.79$.

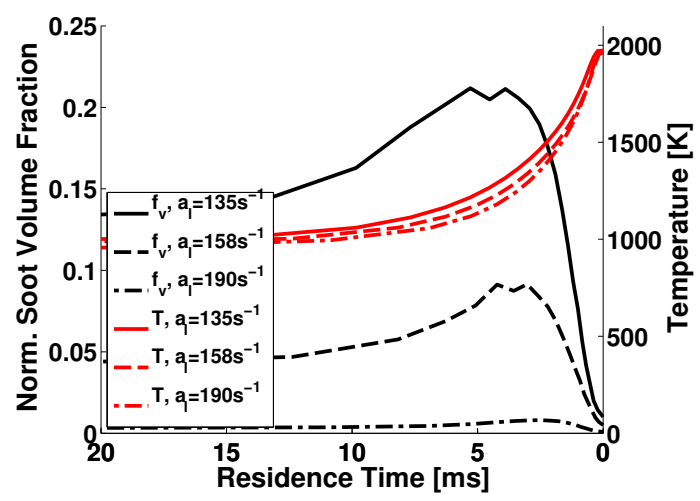

(c) $14 \mathrm{~atm}, X_{\mathrm{C}_{2} \mathrm{H}_{4}}=0.41+X_{\mathrm{He}}=0.59$, $X_{\mathrm{O}_{2}}=0.1723+X_{\mathrm{He}}=0.8277$.

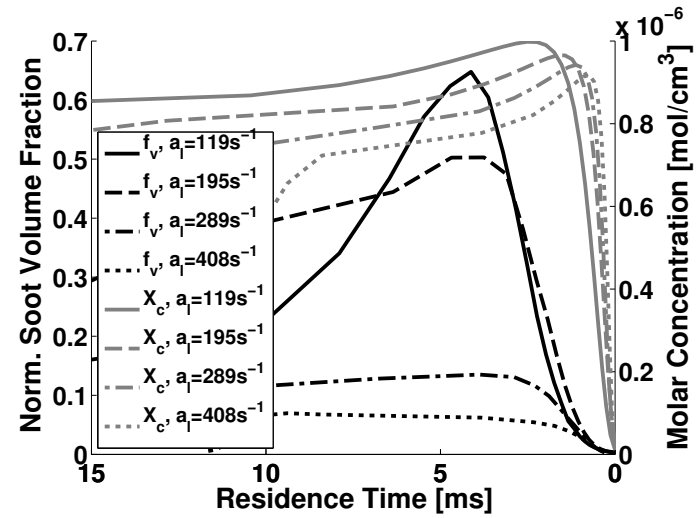

(b) $4.065 \mathrm{~atm}, X_{C_{2} H_{4}}=0.5+X_{N_{2}}=0.5$, $X_{\mathrm{O}_{2}}=0.21+X_{N_{2}}=0.79$.

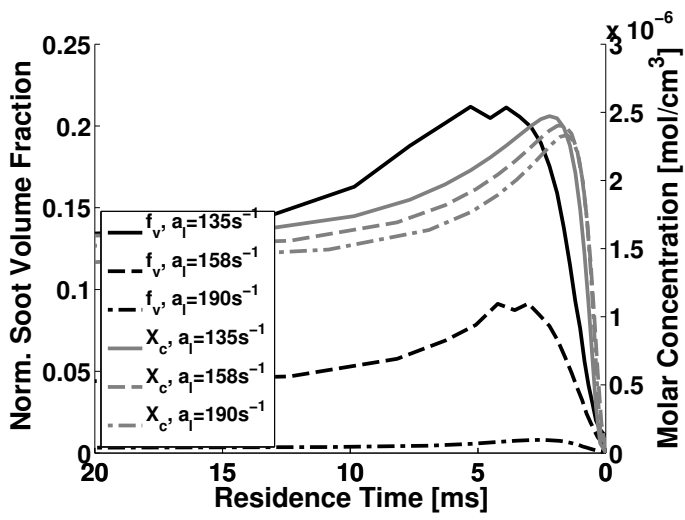

(d) $14 \mathrm{~atm}, X_{C_{2} H_{4}}=0.41+X_{H e}=0.59$, $X_{\mathrm{O}_{2}}=0.1723+X_{\mathrm{He}}=0.8277$.

Figure 4.12: Normalized soot volume fraction as a function of particle residence time for several local strain rates at $4.065 \mathrm{~atm}$ and $14 \mathrm{~atm}$. Red lines: Local gas temperature. Gray lines: $\mathrm{C}_{2} \mathrm{H}_{2}$ molar concentration.

Volume fraction at any point in the soot layer is a function of a particle nucleation rate term, growth rate term, and mass (or volume) sink term from flow divergence in the counterflow configuration. A particle coagulation rate term may also have some effect on reducing available soot surface area for surface mass growth. Evidence suggests the effect is minor [127]. It is possible to make several key observations by analyzing the rates of soot 
volume fraction growth and mass loss inherent in the temporal plots of soot volume fraction in Fig. 4.12. Assuming a constant soot particle density, mass and volume growth of soot are proportional. For simplicity, growth and sink rates will be left in units of normalized volume fraction growth per unit time, specifically $\mathrm{ms}^{-1}$. Figure 4.13 shows the normalized soot volume fraction growth rate (black lines) as a function of particle residence time in the soot layer. Temperature profiles (red lines) are also given for reference. Correcting for the rate of volume fraction lost due to flow divergence is important. The volumetric sink rate due to flowfield divergence is calculated according to $d f_{v} / d t=2 \pi U(z) f_{v}$ where $U(z)$ is the radial velocity gradient parameter and $f_{v}$ the local volume fraction of soot. The sink rate of volume fraction lost is added back to the volume fraction growth rate to correct the total rate of soot volume fraction produced in the flame. The time within which the effect of nucleation dominates volume fraction growth is short. Assuming a nascent soot particle diameter of approximately $5 \mathrm{~nm}$, nucleation is the dominant mechanism controlling the volume fraction growth rate only within the first $0.5 \mathrm{~ms}$ of particle residence time at $4.065 \mathrm{~atm}$. The effect decays to one third of the total volume fraction growth rate within the first millisecond, quickly becoming negligible thereafter. At $14 \mathrm{~atm}$, nucleation is the dominant mechanism in the total volume fraction growth rate within the first $0.1 \mathrm{~ms}$ of particle residence time. The effect decays to one third of the total volume fraction growth rate within the $0.3 \mathrm{~ms}$, becoming negligible thereafter.

First considering the case of $4.065 \mathrm{~atm}, a_{l}=125 \mathrm{~s}^{-1}$ from Fig. $4.13 \mathrm{a}$ ), the soot volumetric growth rate increases from zero to a maximum at a particle residence time of $4 \mathrm{~ms}$ and decays thereafter. For the sample $4.065 \mathrm{~atm}, a_{l}=435 \mathrm{~s}^{-1}$ the volumetric growth rate increases from zero to a maximum at a particle residence time of $1 \mathrm{~ms}$ and decays with increasing residence time. For $14 \mathrm{~atm}, a_{l}=135 \mathrm{~s}^{-1}$ in Fig. $4.13 \mathrm{~b}$ ), the volumetric soot growth rate increases to a maximum at a particle residence time of $4 \mathrm{~ms}$. Lastly, for $14 \mathrm{~atm}$, $a_{l}=190 \mathrm{~s}^{-1}$ the volumetric growth rate increases to a maximum at $2.5 \mathrm{~ms}$ of particle residence. It is accepted that an acetylene driven hydrogen abstraction $\mathrm{C}_{2} \mathrm{H}_{2}$ addition (HACA) 


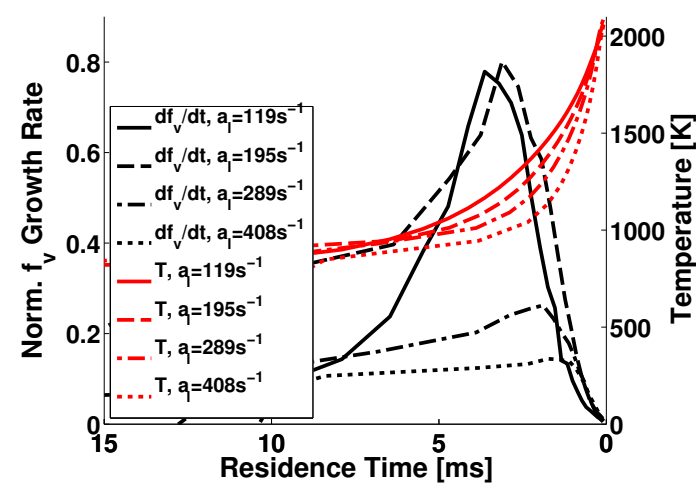

(a) $4.065 \mathrm{~atm}, X_{C_{2} H_{4}}=0.5+X_{N_{2}}=0.5$, $X_{O_{2}}=0.21+X_{N_{2}}=0.79$.

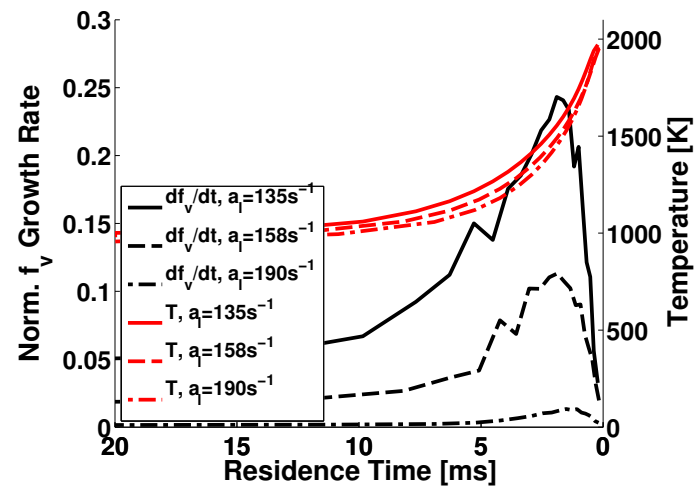

(b) $14 \mathrm{~atm}, X_{\mathrm{C}_{2} \mathrm{H}_{4}}=0.41+X_{H e}=0.59$, $X_{\mathrm{O}_{2}}=0.1723+X_{H e}=0.8277$.

Figure 4.13: Normalized soot volume fraction growth rates as a function of particle residence time for several local strain rates at $4.065 \mathrm{~atm}$ and $14 \mathrm{~atm}$. Red lines: Local gas temperature.

mechanism is a dominant surface mass growth pathway [6,133]. Fig. 4.10 illustrates that few $\mathrm{H}$ atoms are present in the counterflow soot layer indicating that other radicals besides the commonly assumed $\mathrm{H}$ atom are responsible for driving the reaction. The same conclusion was drawn by other authors $[25,144,145]$ with the $\mathrm{H}$ abstraction step postulated to occur via other radicals present in the counterflow soot layer including but not limited to $\mathrm{CH}_{3}, \mathrm{C}_{2} \mathrm{H}$, or $\mathrm{C}_{3} \mathrm{H}_{3}$. Preliminary modeling comparisons with experimental data yielded much improved agreement to trends in soot growth at high temperatures [25]. The reported cutoff temperature of the HACA soot surface growth mechanism is approximately $1400 \mathrm{~K}[6]$. A continuation in volume fraction growth at low temperature around $1000 \mathrm{~K}$ indicates a different surface growth mechanism is active in the region. A PAH-soot surface coagulation growth mechanism has been suggested [6,7,25,48]. Applying both soot growth mechanisms, preliminary modeling results were in agreement with the trend in growth rates at both high and low temperature [25]. Evidence of persistent free radicals contained in the soot particles allowing for surface reactions with gas phase PAH species may also play a role in low temperature soot growth $[6,146]$.

Clearly the rates of soot volume fraction growth change significantly with pressure and strain rate. Examining the rate of volume fraction growth as a function of total particle 
surface area $\left(d f_{v} / d t\right) / 4 \pi d_{p}^{2} N$, it is possible to narrow down the cause of the change in the volume fraction growth rate. For all strain rate cases at 4.065 atm and 14 atm the volume fraction growth rates as a function of total surface area increase to a consistent maximum rate early in the high temperature region and decay by approximately an order of magnitude within $10 \mathrm{~ms}$ in the low temperature region around $1000 \mathrm{~K}$. The growth rates exhibit a clear temperature dependency. Uncertainties are significant for the volumetric growth rate per surface area estimation, but the general trend is consistent. The insensitivity of volumetric growth rates as a function of surface area to pressure and strain rate was also consistent with data at other pressures beyond $4.065 \mathrm{~atm}$ and $14 \mathrm{~atm}$. The results indicate that pressure and strain do not strongly affect soot surface growth rates. The rate of volume fraction growth is primarily from surface growth mechanisms proportionally magnified by the number density of soot in the region providing the surface area foundation. Thus the pressure and strain or residence time effects dictating the total soot volume fraction produced in a flame are mostly manifested in the kinetic mechanism of soot nucleation. Charest et al. [147] drew differing conclusions using a laminar co-flow diffusion burner. Changes in soot loading were found primarily due to nucleation and the time available to the nucleation mechanism.

Lastly, the uncertainties associated with the estimated rates of volume fraction growth are estimated. The dominant uncertainties result from an uncertainty in the volume fraction measurement and uncertainty in the thermal conductivity of soot dictating the thermophoretic force and soot particle residence time. Soot volume fraction measurement uncertainty is approximately $30 \%$ for all flames. Residence time uncertainty is estimated as approximately $15 \%$ not accounting for unknown uncertainties in gas velocity from numerical modeling. The total uncertainty associated with the volume fraction growth rate is estimated at $34 \%$. 


\subsection{Primary Soot Particle Diameter}

Figure 4.14 shows normalized soot primary particle diameter profiles extracted from the measured incandescent signal temporal decay using LII. The plots illustrate that the soot particles are nucleated near the maximum flame temperature on the fuel rich side, are convected down the gas temperature gradient due to a combination of Stokes drag from the gas velocity and a thermophoretic force from the strong temperature gradient in the sooting region. As the particles travel through the reacting layer, they continuously grow in size due to soot surface growth mechanisms and particle-particle collision and coagulation from Brownian motion $[6,148]$. Initial particle growth is initiated by small nascent soot particle coagulation from collisions and chemical kinetic surface growth smoothing the surface resulting in primary particles reasonably spherical in shape [148]. The particles reach a maximum size as they approach the particle stagnation plane where a convective sink carries them out of the centerline measurement region due to the divergence in the flow field.

LII derived primary particle diameter exhibits a total uncertainty of $44 \%$ for a helium diluted flame and $50 \%$ for a nitrogen diluted flame. The large range of particle sizes observed during particle growth in the soot region allow for useful trends to be established even when taking into account the large uncertainty of the particle diameter measurement. Aggregate soot particles made of many primary particle spheroids are observed obeying a consistent fractal scaling [75] with increasing residence time for continued particle-particle collisions and reduced surface growth [148]. In general however, particle residence times in the counterflow flame on the order of $10 \mathrm{~ms}$ are low enough to inhibit significant formation of fractal aggregate soot particles. This is not the case for premixed burners driven by buoyancy where residence times are several tens of milliseconds and significant aggregation is observed. Data at $8.45 \mathrm{~atm}$ under high soot loading and measurements at $30 \mathrm{~atm}$ exhibit soot particles past the estimated particle stagnation plane. Measurements at 20 atm are also slightly affected. This indicates potential unquantifiable errors in calculating temperature, 
velocity, and species profiles inherent in the numerical model or a poor soot thermal conductivity assumption in the affected results. Data at 8.45, 20, and 30 atm all indicate large maximum particle sizes beyond the typical regime of primary soot particles. A spherical particle assumption is considered valid up to approximately $50 \mathrm{~nm}$ in size [149]. The results are indicative of some aggregation that may exist near particle stagnation in the final stages of soot particle growth. Indeed, small aggregate particles from thermophoretic sampling at $8.45 \mathrm{~atm}$ are shown in Fig. 4.1. Evidence in literature suggests aggregates experience a stronger thermophoretic force than primary particles and may also partially explain under high soot loading and larger soot particles why they are observed beyond the calculated particle stagnation plane [127].

At first glance in Fig. 4.14, the lack of change in maximum particle size with strain rate is counterintuitive. Examining the normalized particle diameter profiles in residence time space provides some answers. Fig. 4.15 illustrates the continuous growth in particle diameter up to the particle stagnation plane as a function of particle residence time along the axial centerline of the counterflow burner. The black lines represent normalized soot particle diameter profiles over a range of strain rates. The same strain rates and pressures of $4.065 \mathrm{~atm}$ and $14 \mathrm{~atm}$ are presented as above. Local gas temperature profiles (red lines) are also shown in Fig. 4.15 a) and c) with $\mathrm{C}_{2} \mathrm{H}_{2}$ concentration profiles (gray lines) in Fig $4.15 \mathrm{~b})$ and d) as the primary species governing soot surface reactions [6,133] especially at high temperatures. The figures indicate that even though strain rate is varied, particle residence time remains mostly unchanged due to a squeezing effect at higher strain rates pushing more of the soot layer closer to the stagnation plane. The results then suggest that particle surface growth rates must be similar with temperature over a range of strain rates. Looking more carefully at Fig. 4.15 reveals two unique regimes of surface growth. A more rapid growth period is observed at high temperature and early residence time within 3-5 ms at $4.065 \mathrm{~atm}$ and approximately $6 \mathrm{~ms}$ at $14 \mathrm{~atm}$. Increased particle growth occurs earlier with increasing strain rate, likely due to the temporal shift of precursor species towards 

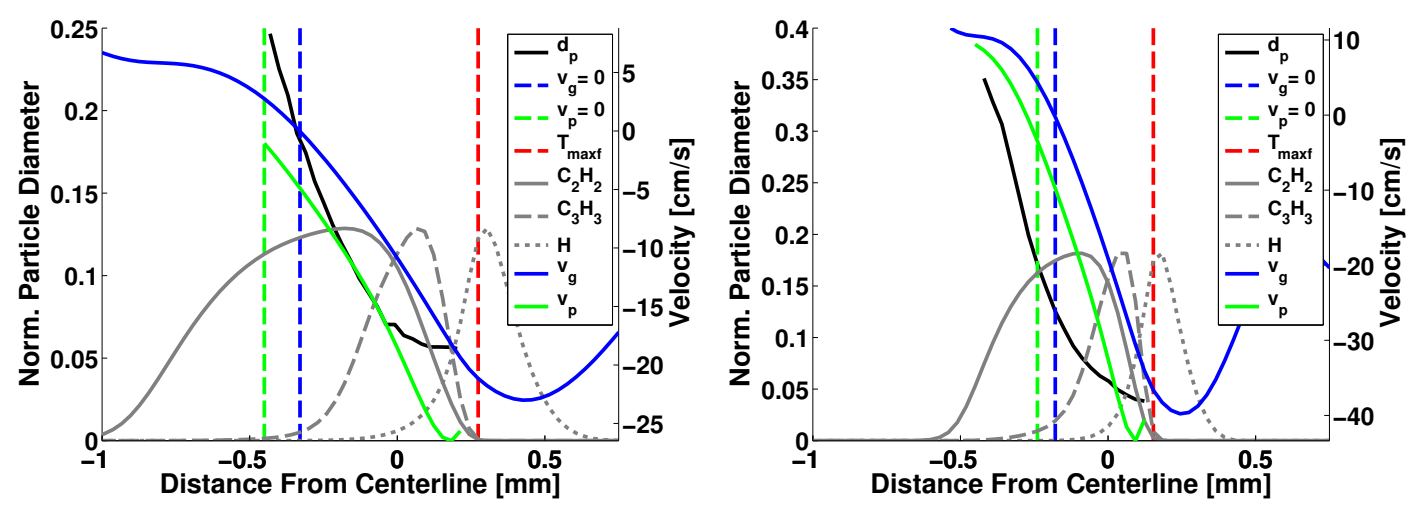

(a) $4.065 \mathrm{~atm}, a_{l}=119 \mathrm{~s}^{-1}, X_{C_{2} H_{4}}=0.5+$ $X_{N_{2}}=0.5, X_{O_{2}}=0.21+X_{N_{2}}=0.79$.

(b) $4.065 \mathrm{~atm}, a_{l}=408 \mathrm{~s}^{-1}, X_{C_{2} H_{4}}=0.5+$

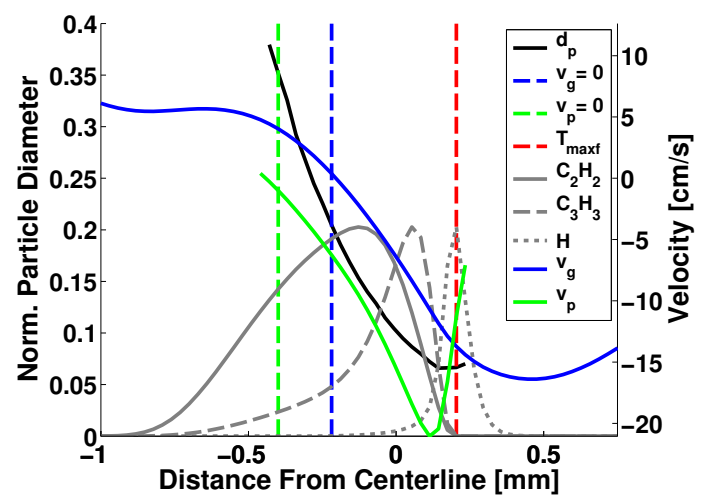

$X_{N_{2}}=0.5, X_{O_{2}}=0.21+X_{N_{2}}=0.79$.

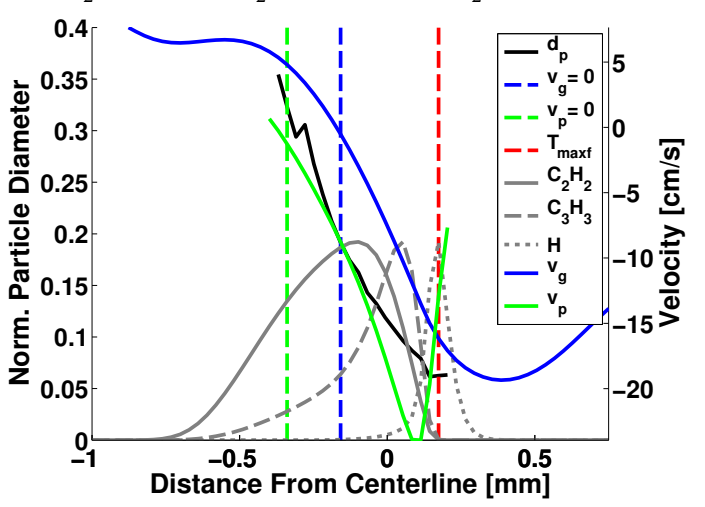

(c) $14 \mathrm{~atm}, a_{l}=135 \mathrm{~s}^{-1}, X_{C_{2} H_{4}}=0.41+$ $X_{H e}=0.59, X_{O_{2}}=0.1723+X_{H e}=$ 0.8277 .

(d) $14 \mathrm{~atm}, a_{l}=190 \mathrm{~s}^{-1}, X_{C_{2} H_{4}}=0.41+$ $X_{H e}=0.59, X_{O_{2}}=0.1723+X_{H e}=$ 0.8277 .

Figure 4.14: Normalized soot primary particle diameter for several local strain rates at $4.065 \mathrm{~atm}$ and $14 \mathrm{~atm}$. Dashed red line: Point of maximum flame temperature. Gray lines: Normalized soot precursors $\mathrm{C}_{2} \mathrm{H}_{2}, \mathrm{C}_{3} \mathrm{H}_{3}$, and $\mathrm{H}$. Blue line: Local gas velocity. Dashed blue line: Gas stagnation. Green line: Local particle velocity. Dashed green line: Particle stagnation.

shorter residence time providing increased $\mathrm{C}_{2} \mathrm{H}_{2}$ concentration to drive surface growth. The rapid surface growth region is halted earlier with increasing strain rate due to the drop in local temperature, possibly below a level where a high temperature HACA surface growth mechanism is active $[6,25,48]$. A slower surface growth period in the low temperature region around $1000 \mathrm{~K}$ is observed thereafter at both pressures in agreement with the nonzero soot volume fraction growth rates previously determined at low temperature and long particle residence times. Evidence suggests that low temperature particle growth is governed by surface growth and not particle coagulation for $4.065 \mathrm{~atm}$ and $14 \mathrm{~atm}$ but may 
play a larger role at higher pressures. Coagulation processes are ongoing throughout the entire soot lifecycle dependent on pressure and particle size i.e. Knudsen number, local gas properties, local temperature, and the distribution and concentration of soot particles in the region [140]. Soot number density changes significantly with strain rate while negligible changes in particle size growth are observed in the two cases presented. Thus the order of magnitude of the particle coagulation term on particle size growth is likely negligible at $4.065 \mathrm{~atm}$ and $14 \mathrm{~atm}$. Additional evidence can be built when considering particle collision dynamics. Collision frequencies are highest between small particles coagulating on large particle surfaces [6]. Small particle coagulation is unlikely to greatly affect particle surface growth due to their small volume. The same argument was proven valid for the effect of nucleation on volume fraction growth previously. Detailed modeling would better determine the magnitudes of all particle growth mechanisms especially at higher pressures.

Figure 4.16 shows the normalized primary soot particle volumetric growth rate (black lines) as a function of particle residence time in the soot layer. Temperature profiles (red lines) are also given for reference. First considering the case of $4.065 \mathrm{~atm}, a_{l}=125 \mathrm{~s}^{-1}$ from Fig. 4.16 a), the normalized primary soot volumetric growth rate increases from zero to a maximum at a particle residence time of approximately $6 \mathrm{~ms}$. The rate then decays by nearly a factor of three within a particle residence time of $10 \mathrm{~ms}$. For the sample $4.065 \mathrm{~atm}$, $a_{l}=435 \mathrm{~s}^{-1}$ the soot particle volumetric growth rate increases from zero to the same maximum at a particle residence time of $2 \mathrm{~ms}$ and decays down around the same minimum within $10 \mathrm{~ms}$. For $14 \mathrm{~atm}, a_{l}=135 \mathrm{~s}^{-1}$ in Fig. $4.16 \mathrm{~b}$ ), the soot particle volumetric soot growth rate increases to a maximum at a particle residence time of $7 \mathrm{~ms}$ then decays by a factor of three within $15 \mathrm{~ms}$. Lastly, for $14 \mathrm{~atm}, a_{l}=190 \mathrm{~s}^{-1}$ the volumetric growth rate increases to a maximum at $4 \mathrm{~ms}$ and decreases by a factor of four within $15 \mathrm{~ms}$. Considering the uncertainties in the growth estimates, the growth profiles should be considered consistent. Primary particle diameter volumetric growth is observed active at low temperatures in agreement with volume fraction growth rates shown previously in Fig. 4.13. The results 

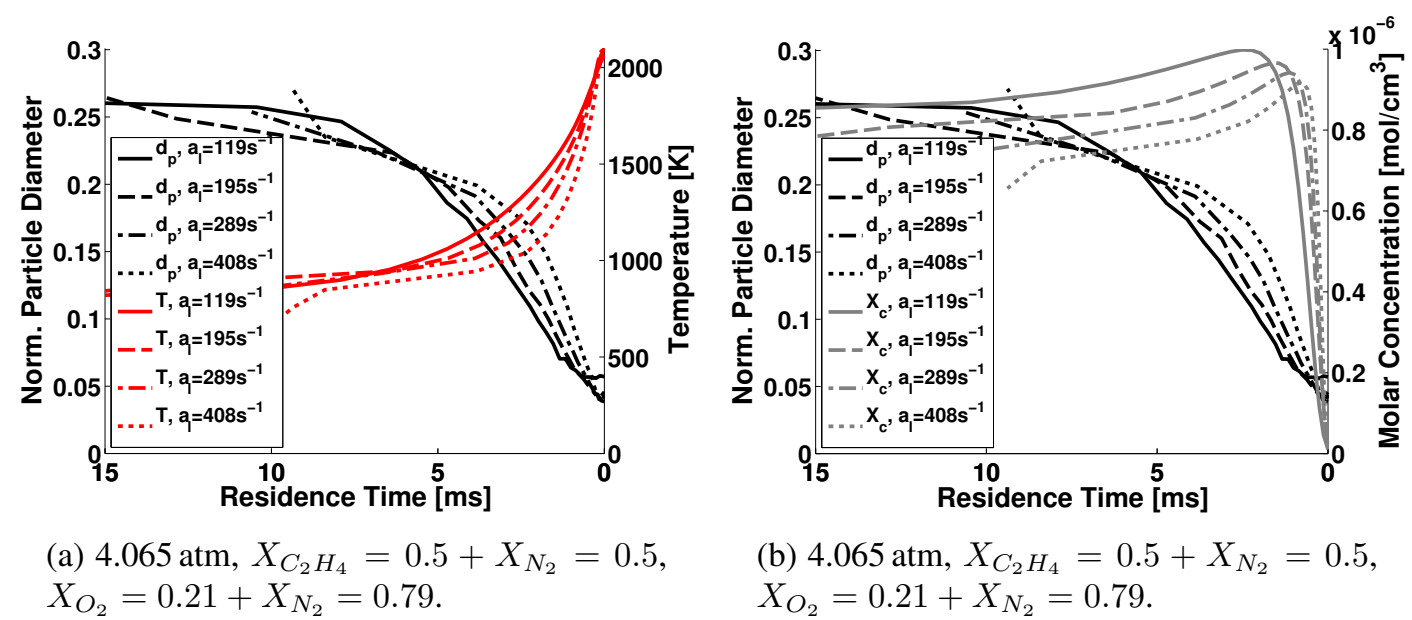

(b) $4.065 \mathrm{~atm}, X_{C_{2} H_{4}}=0.5+X_{N_{2}}=0.5$, $X_{O_{2}}=0.21+X_{N_{2}}=0.79$.

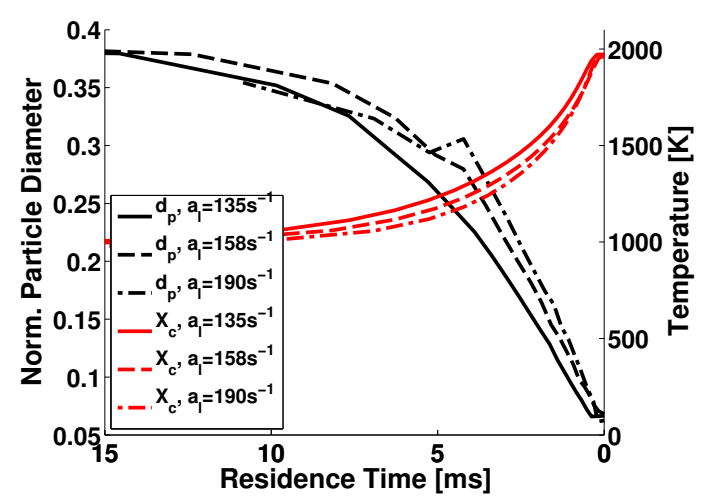

(c) $14 \mathrm{~atm}, X_{\mathrm{C}_{2} \mathrm{H}_{4}}=0.41+X_{\mathrm{He}}=0.59$, $X_{\mathrm{O}_{2}}=0.1723+X_{\mathrm{He}}=0.8277$.

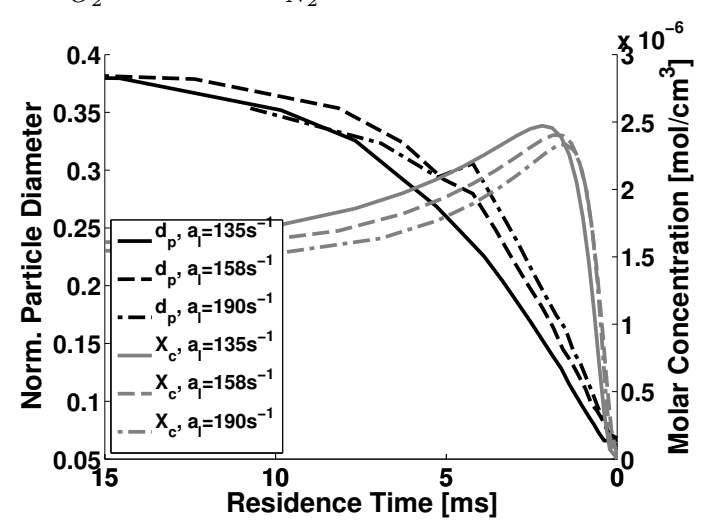

(d) $14 \mathrm{~atm}, X_{\mathrm{C}_{2} \mathrm{H}_{4}}=0.41+X_{\mathrm{He}}=0.59$, $X_{O_{2}}=0.1723+X_{H e}=0.8277$.

Figure 4.15: Normalized primary soot particle diameter as a function of particle residence time for several local strain rates at $4.065 \mathrm{~atm}$ and $14 \mathrm{~atm}$. Red lines: Local gas temperature. Gray lines: $\mathrm{C}_{2} \mathrm{H}_{2}$ molar concentration.

further prove that a low temperature growth mechanism is active beyond the typical high temperature HACA mechanism.

The rate of normalized primary soot particle volumetric growth as a function of total particle surface area is given by the black lines in Fig. 4.17 for the same strain rate cases at $4.065 \mathrm{~atm}$ and $14 \mathrm{~atm}$. Temperature profiles are included in red. The profiles contain the lowest possible uncertainty for an estimate of volumetric growth as a function of surface area due to independence on volume fraction and number density and their respective uncertainties. A comparison of all cases shown indicate that soot volume growth as a function of particle surface area is relatively insensitive to changes in pressure and strain rate. 


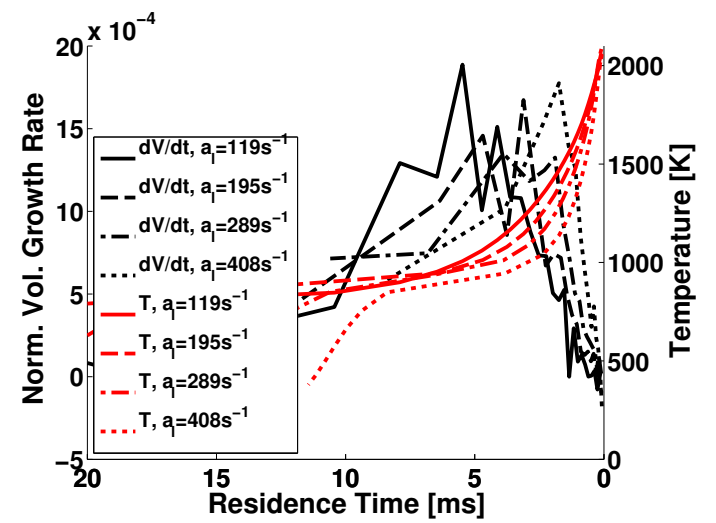

$$
\text { (a) } 4.065 \mathrm{~atm}, X_{C_{2} H_{4}}=0.5+X_{N_{2}}=0.5 \text {, }
$$
$X_{\mathrm{O}_{2}}=0.21+X_{N_{2}}=0.79$.

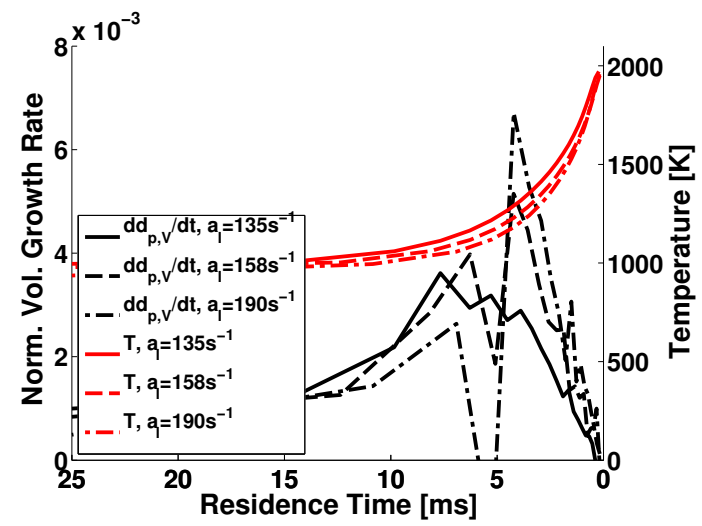

(b) $14 \mathrm{~atm}, X_{\mathrm{C}_{2} \mathrm{H}_{4}}=0.41+X_{\mathrm{He}}=0.59$, $X_{O_{2}}=0.1723+X_{H e}=0.8277$.

Figure 4.16: Normalized primary soot particle volumetric growth rates as a function of particle residence time for several local strain rates at $4.065 \mathrm{~atm}$ and $14 \mathrm{~atm}$. Red lines: Local gas temperature.

The magnitudes of volumetric surface growth also retain the same proportionality with temperature. Growth rates are maximum at early particle residence times and decay with increasing residence time and decreasing temperature until the particle stagnation plane. Volumetric growth rates as a function of surface area were also tested for consistency at higher and lower pressures beyond the $4.065 \mathrm{~atm}$ and $14 \mathrm{~atm}$ cases presented. All results exhibited the same magnitudes and temperature dependency. The phenomenon is in qualitative agreement with both chemical HACA and physical PAH coagulation mechanisms which both exhibit temperature dependencies $[25,133]$. The intriguing results reinforce the hypothesis that the rate of soot nucleation is the driver of the total soot volume produced and is strongly affected by temperature, residence time, and pressure. The surface growth rate remains relatively constant with changes in strain rate and pressure and is activated on the number of nascent soot particles produced in the flame. Future modeling efforts should focus on soot nucleation for maximum impact.

The uncertainty associated with the estimated rates of soot primary particle diameter growth includes uncertainty in the primary particle diameter measurement and soot particle residence time. Measurement uncertainty is approximately $40 \%$ for helium-diluted flames and 50\% for nitrogen-diluted flames. Residence time uncertainty is estimated as 


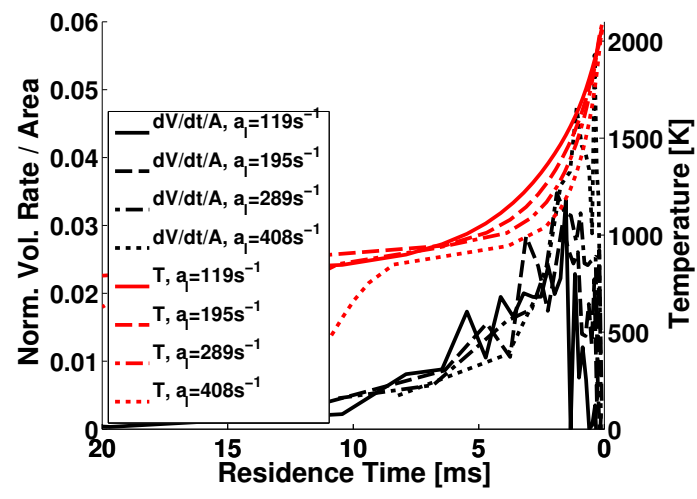

(a) $4.065 \mathrm{~atm}, X_{C_{2} H_{4}}=0.5+X_{N_{2}}=0.5$, $X_{O_{2}}=0.21+X_{N_{2}}=0.79$.

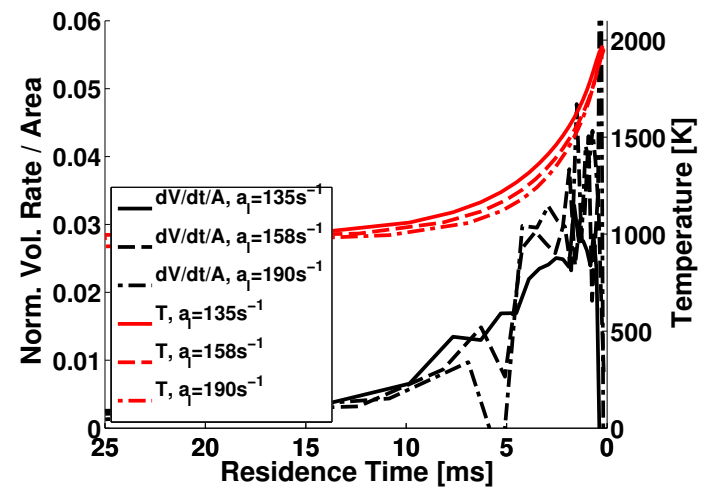

(b) $14 \mathrm{~atm}, X_{\mathrm{C}_{2} \mathrm{H}_{4}}=0.41+X_{H e}=0.59$, $X_{\mathrm{O}_{2}}=0.1723+X_{\mathrm{He}}=0.8277$.

Figure 4.17: Normalized primary soot particle volumetric growth rates per surface area as a function of particle residence time for several local strain rates at $4.065 \mathrm{~atm}$ and $14 \mathrm{~atm}$. Red lines: Local gas temperature.

approximately $15 \%$ not accounting for unknown uncertainties in gas velocity from numerical modeling. Measurement uncertainty is dominant resulting in total uncertainties for primary particle diameter growth of $43 \%$ for helium-diluted flames and $52 \%$ for nitrogendiluted flames. Additional uncertainties apply to estimated volume growth rates. Assuming a monodisperse particle diameter distribution when calculating the particle surface volume growth rate introduces an error of approximately $12 \%$. Assuming a lognormal distribution with $\sigma_{d_{p, g}}=1.19$ removes most of the error owing to the mean of both distributions being nearly identical as solutions to the LII model. Regardless of the chosen distribution assumption, the uncertainty from the distribution parameter remains negligible. Volumetric particle growth rates per particle surface area are $44 \%$ and $54 \%$ for helium-diluted and nitrogen-diluted flames respectively. Volumetric particle growth rates are $72 \%$ and $89 \%$ for helium-diluted and nitrogen-diluted flames respectively.

\subsection{Elastic Light Scattering Results}

The majority of LII data collected exhibited soot particle sizes in the regime of primary particles with the exception of data at $8.45,20$, and 30 atm which all indicated large po- 
tentially aggregate particles were present. A spherical particle assumption is considered valid up to approximately $50 \mathrm{~nm}$ in size [149]. The results at 8.45, 20, and 30 atm all show some indication of aggregation in the final stages of soot particle growth. Additional evidence is shown in Fig. 4.1 by the small aggregate particles from thermophoretic sampling at $8.45 \mathrm{~atm}$. Elastic light scattering (ELS) data was collected and the combined LII and ELS model outlined in Chapter 2.4 was applied to datasets with indications of aggregation. Figure 4.18 show particle temperature and normalized soot volume fraction, primary particle size, and number density extracted without the ELS submodel at a pressure of $8.45 \mathrm{~atm}$, $a_{l}=163 \mathrm{~s}^{-1}, X_{C_{2} H_{4}}=0.46+X_{N_{2}}=0.64, X_{O_{2}}=0.1933+X_{N_{2}}=0.8067$. The same definitions apply as in all other spatial figures of LII variables presented in this chapter. Most notably, the maximum particle diameter is beyond the approximate limit of spherical primary particles. More importantly, a strong ELS signal was measured indicating the potential for aggregation. Applying the combined LII and ELS model to the same dataset at $8.45 \mathrm{~atm}, a_{l}=163 \mathrm{~s}^{-1}, X_{C_{2} H_{4}}=0.46+X_{N_{2}}=0.64, X_{O_{2}}=0.1933+X_{N_{2}}=0.8067$ resulted in the measurements given in Fig. 4.19. Particle temperature remained unchanged so the normalized number of primary particles per aggregate $N_{p}$ was given instead in Fig. 4.19 a). The data resulted in a general trend of the number of primary particles per aggregate relatively small near the maximum flame temperature and particle nucleation. The number of primary particles per aggregate then increase to a maximum near particle stagnation. The primary particle diameter profile is altered and the maximum primary particle size is significantly reduced. The number of primary particle per aggregate and maximum primary particle sizes are in general agreement with TEM data analyzed in Chapter 4.1. The combined LII and ELS model also have a noticable effect on the extracted soot volume fraction and number density when comparing Figs. 4.18 and 4.19. The data should be scrutinized with caution however, as many challenges were discovered during the fitting procedure. The ELS error surface generally exhibited no definitive minimum and instead gave a leveled off low error region. The LII error surface provided the error minimum 


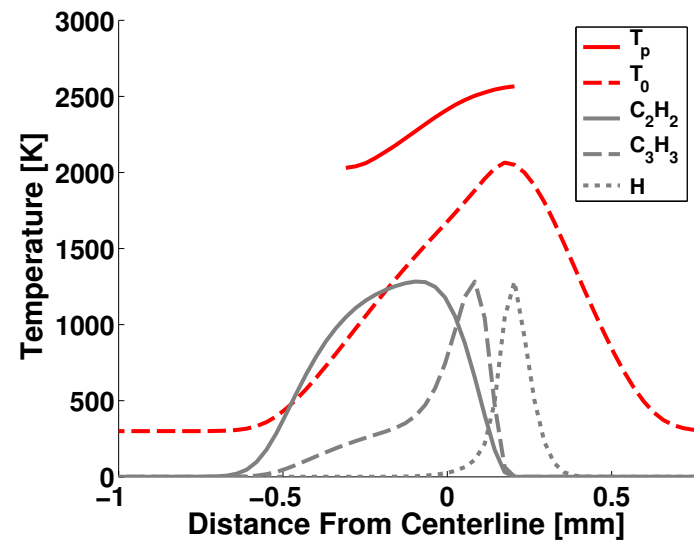

(a) Particle and gas temperature.

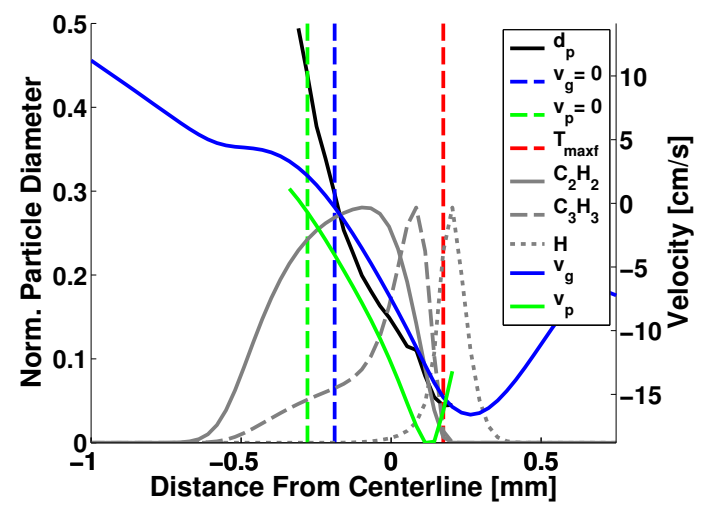

(c) Primary particle diameter.

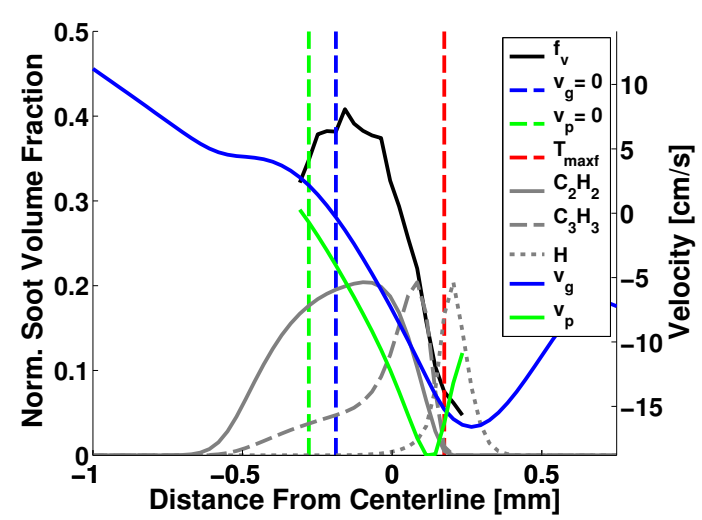

(b) Volume fraction.

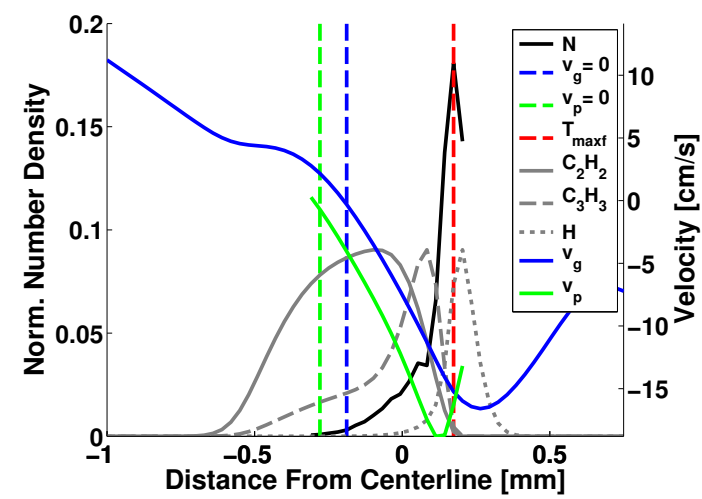

(d) Number density.

Figure 4.18: LII extracted measurements of a) particle temperature (red line) and modeled gas temperature (red dashed profile), b) normalized soot volume fraction, c) normalized primary particle diameter, and d) normalized number density at $8.45 \mathrm{~atm}, a_{l}=163 \mathrm{~s}^{-1}$, $X_{C_{2} H_{4}}=0.46+X_{N_{2}}=0.64, X_{O_{2}}=0.1933+X_{N_{2}}=0.8067$. Vertical dashed red line: Point of maximum flame temperature. Gray lines: Normalized soot precursors $\mathrm{C}_{2} \mathrm{H}_{2}$, $\mathrm{C}_{3} \mathrm{H}_{3}$, and $\mathrm{H}$. Blue line: Local gas velocity. Vertical dashed blue line: Gas stagnation. Green line: Local particle velocity. Vertical dashed green line: Particle stagnation.

where the two surfaces met. This phenomenon was at odds with ELS data collected on a premixed flat flame burner which exhibited a clear minimum. The result indicates poor characterization of aggregate properties from counterflow diffusion flames using the ELS procedure outlined in Chapter 2.4. Elastic light scattering data collected at 20 and $30 \mathrm{~atm}$ was of even poorer quality and lacked sufficient signal to attempt a successful combined LII and ELS model data extraction. Similar results were reported in literature [9] on diffusion flames. The issues were associated with strong morphology and concentration gradients inherent to diffusion flames. Wide angle light scattering was proposed $[129,150]$ as a po- 


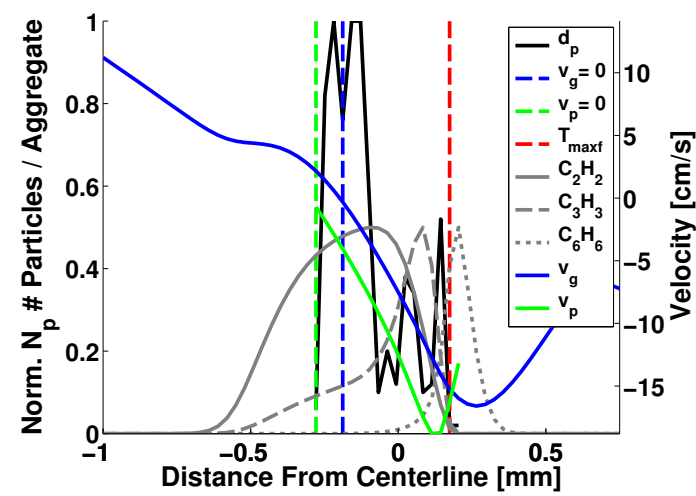

(a) Number of primary particles per aggregate.

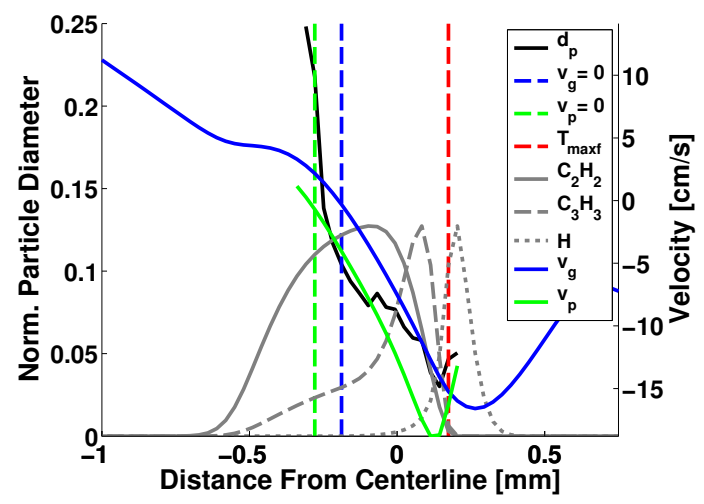

(c) Primary particle diameter.

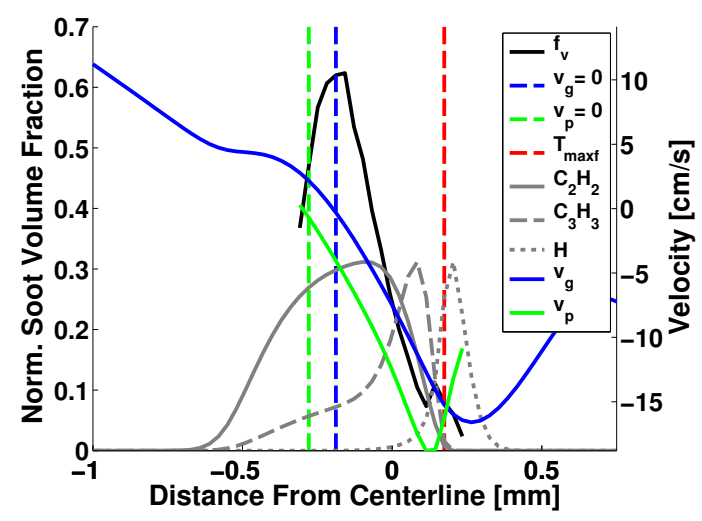

(b) Volume fraction.

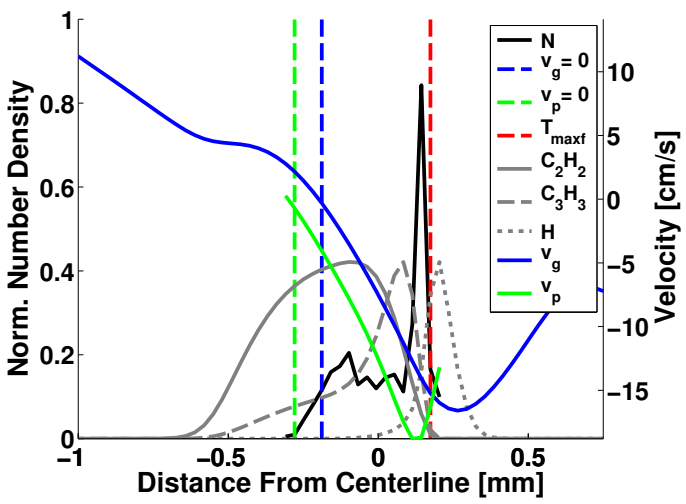

(d) Number density.

Figure 4.19: LII and ELS extracted measurements of a) normalized number of primary particles per aggregate, b) normalized soot volume fraction, c) normalized primary particle diameter, and d) normalized number density at $8.45 \mathrm{~atm}, a_{l}=163 \mathrm{~s}^{-1}, X_{C_{2} H_{4}}=0.46+$ $X_{N_{2}}=0.64, X_{O_{2}}=0.1933+X_{N_{2}}=0.8067$. Dashed red line: Point of maximum flame temperature. Gray lines: Normalized soot precursors $\mathrm{C}_{2} \mathrm{H}_{2}, \mathrm{C}_{3} \mathrm{H}_{3}$, and $\mathrm{H}$. Blue line: Local gas velocity. Dashed blue line: Gas stagnation. Green line: Local particle velocity. Dashed green line: Particle stagnation.

tential solution and improvement over the single angle scattering procedure developed in this study with encouraging results.

\subsection{Sooting Limits}

Accurate prediction of soot nucleation poses the greatest challenge to the scientific community $[6,133]$. Mounting evidence from this study suggests the process of soot nucleation is the primary mechanism driving the amount of soot produced in counterflow diffusion 
flames. Some consensus on the theory of the importance of soot nucleation has developed for other burner configurations as well $[7,147]$. The counterflow burner is particularly valuable for targeting uncertain nucleation processes by adjusting flow strain rate and fueloxidizer composition. The limiting flow strain or residence time at which no perceptible soot is formed is an especially useful metric for testing the sensitivity and accuracy of soot nucleation models. There are however, several caveats that must be regarded when attempting to identify sooting limits. The number density of nascent soot formed in the counterflow flame was shown in Fig. 4.7 to exhibit an exponential dependency on temperature in accordance with an Arrhenius global reaction rate description. Figure 4.11 also illustrated the linear dependency between number density and soot volume fraction. The number density of nascent soot serves as the foundation for soot mass and volume growth in the counterflow burner used in this study. This leads to the conclusion that soot limits should not be linearly extrapolated from trends of volume fraction at lightly sooting conditions, a technique that appears plausible when examining soot volume fraction alone without heed to the underlying relationships. Instead a sooting limit can be identified based on any target volume fraction where formation of soot below the stated target is deemed acceptable for a specific application. An extrapolation of the exponential decay of soot volume fraction with respect to strain rate can then be performed. Choosing a target of zero is not strictly possible by definition. A target sooting limit was chosen representative of the approximate sensitivity limit of the LII system developed in this study. Fig. 4.20 illustrates the relationship between maximum soot volume fraction and local strain rate with changes in pressure. A clear trend representative of an exponential decay is observed at all pressures. Marked changes in the relationship between maximum soot volume fraction and local strain rate are also evident with changes in pressure. The slopes of the curves increase with increasing pressure. Not accounting for changes in species composition, the slopes indicate an increased sensitivity on the formation of soot volume fraction with increasing pressure due to a proportional relationship between strain rate and temperature. A decrease in volume 
fraction and local strain rate with increasing pressure is also evident, however temperature and species concentration effects also play a role. The results are indicative of a greater barrier to soot formation at higher pressure in accordance with the global activation energy arguments proposed from Fig. 4.7.

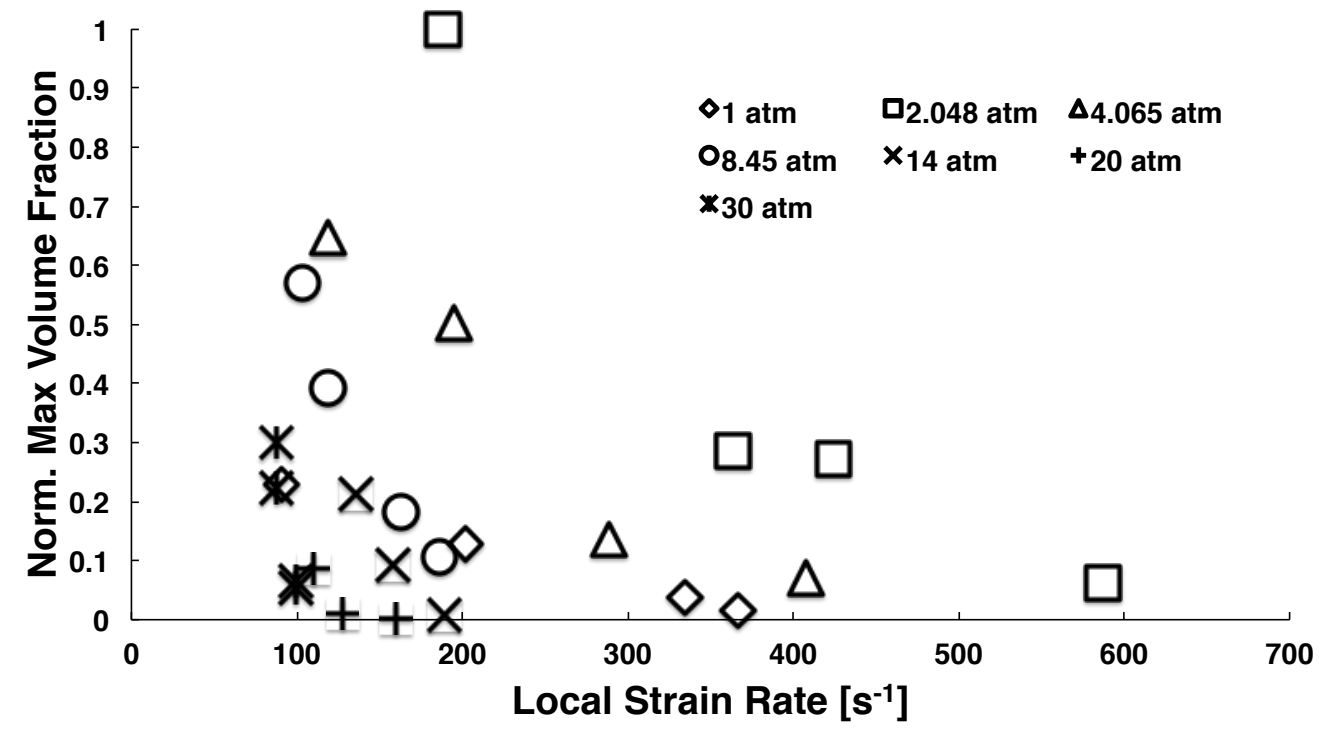

Figure 4.20: LII measured normalized maximum soot volume fraction as a function of local strain rate over a range of pressures from 1 to $30 \mathrm{~atm}$. Diamonds: $1 \mathrm{~atm}, a_{l}=$ $91,202,335,367 \mathrm{~s}^{-1}, X_{C_{2} H_{4}}=1, X_{O_{2}}=0.21+X_{N_{2}}=0.79$. Squares: $2.048 \mathrm{~atm}$, $a_{l}=188,210,364,424,587 \mathrm{~s}^{-1}, X_{C_{2} H_{4}}=1, X_{O_{2}}=0.21+X_{N_{2}}=0.79$. Triangles: $4.065 \mathrm{~atm}, a_{l}=119,195,289,408 \mathrm{~s}^{-1}, X_{C_{2} H_{4}}=0.5+X_{N_{2}}=0.5, X_{O_{2}}=0.21+X_{N_{2}}=$ 0.79. Circles: $8.45 \mathrm{~atm}, a_{l}=104,119,163,186 \mathrm{~s}^{-1}, X_{C_{2} H_{4}}=0.4+X_{N_{2}}=0.6, X_{O_{2}}=$ $0.1681+X_{N_{2}}=0.8319$. X: $14 \mathrm{~atm}, a_{l}=135,158,190 \mathrm{~s}^{-1}, X_{C_{2} H_{4}}=0.41+X_{H e}=0.59$, $X_{\mathrm{O}_{2}}=0.1723+X_{H e}=0.8277$. Cross: $20 \mathrm{~atm}, a_{l}=110,128,160 \mathrm{~s}^{-1}, X_{C_{2} H_{4}}=$ $0.4+X_{H e}=0.6, X_{O_{2}}=0.1681+X_{H e}=0.8319$. Double Cross: $30 \mathrm{~atm}, a_{l}=88,100 \mathrm{~s}^{-1}$, $X_{C_{2} H_{4}}=0.39+X_{H e}=0.61, X_{O_{2}}=0.1639+X_{H e}=0.8361$.

The soot limits were estimated as $819 \mathrm{~s}^{-1} \pm 31 \%$ at $1 \mathrm{~atm}, 1370 \mathrm{~s}^{-1} \pm 23 \%$ at $2.048 \mathrm{~atm}$, $1044 \mathrm{~s}^{-1} \pm 30 \%$ at $4.065 \mathrm{~atm}, 481 \mathrm{~s}^{-1} \pm 42 \%$ at $8.45 \mathrm{~atm}, 245 \mathrm{~s}^{-1} \pm 91 \%$ at $14 \mathrm{~atm}, 171 \mathrm{~s}^{-1}$ $\pm 124 \%$ at $20 \mathrm{~atm}$, and $140 \mathrm{~s}^{-1} \pm 26 \%$ at $30 \mathrm{~atm}$. Uncertainty was estimated from the uncertainty in the local strain rate and volume fraction measurements. The data was linearized by the natural log of volume fraction and a linear regression was applied. A Monte Carlo approach was used where random samples were produced from the standard uncertainties in each measurement. Five hundred random samples were generated and the standard de- 
viation in the regression coefficients was calculated on the samples. Uncertainty in the soot limit strain rate was estimated according to $\Delta a_{l} / a_{l}=\sqrt{\left(\Delta \beta_{1} / \beta_{1}\right)^{2}+\left(\Delta \beta_{0} / \beta_{0}\right)^{2}}$. Where $\beta_{1}$ and $\beta_{0}$ are the first and zeroth order regression coefficients. The results at 20 atm exhibited an uncertainty above $100 \%$ primarily due to the close proximity of the data points in the sample. A negative soot limit strain rate is not physically possible so the soot limit strain rate range should technically be considered between 0 and $383 \mathrm{~s}^{-1}$ at $20 \mathrm{~atm}$. Maximum temperature limits corresponding to the determined strain rate limits are in a range between $1800 \mathrm{~K}$ and $1950 \mathrm{~K}$. Conclusions drawn from strain rate limits are complicated due to varying temperature, species, and flame structure effects with strain rate, pressure, and diluent. However, several observations can be made. Law et al. [10] suggested a linear pressure scaling between density weighted strain rate and fuel concentration according to $\rho_{o x} a \propto p_{0} X_{f}^{0.5}$ up to the $2.5 \mathrm{~atm}$ limit of pressure investigated. $\rho_{o x}$ corresponds to the density of the oxidizer stream mixture, $a$ a local or global strain rate, $X_{f}$ the fuel mole fraction, and $p_{0}$ corresponding to pressure. Interestingly, this scaling holds true in this work up to 4.065 atm but diverges significantly thereafter. Soot limit strain rates initially rise from $1 \mathrm{~atm}$ to between $2.048 \mathrm{~atm}$ and 4.065 atm and consistently drop with increasing pressure thereafter even after accounting for uncertainties. The decreasing trend in soot limits levels off with pressure past 14 atm. Probing soot limits required adjusting fuel-oxidizer concentrations and strain rates to maintain flames below a turbulent limit Reynolds number of approximately 2600. Thus fuel-oxidizer settings could not be maintained constant over all pressures. Regardless of the constraints, fuel-oxidizer mole fractions remain nearly constant between 8.45 and 30 atm and likely cannot account for the changes in soot limit strain rate. The maximum soot limit strain rates at $2.048 \mathrm{~atm}$ and $4.065 \mathrm{~atm}$ indicate the greatest ease in forming soot out of all pressures by requiring the highest strain rates and shortest residence times to halt soot production. The changes in soot loading with pressure and strain rate using a counterflow burner clearly indicate the effect of pressure on soot loading is not due to an increase in residence time to form soot as concluded by Charest et 
al. [147] using a co-flow diffusion burner. Data at higher pressure was taken at lower strain rates indicating additional time available to form soot at higher temperatures and yet nucleation rates are reduced. The effect of pressure on soot nucleation and total soot volume fraction produced appears chemical kinetic in nature. The evidence is in agreement with global activation energies determined earlier from Fig. 4.7. Increasing activation energies with increasing pressure indicates higher temperatures are required for soot nucleation to commence. 


\section{Chapter 5}

\section{Summary and Recommendations}

The societal need for energy is ever-present. Hydrocarbon combustion has and will continue to support a large portion of global demand. The numerous health and environmental risks associated with soot formed from hydrocarbon combustion have driven research into formation and growth mechanisms with emphasis on developing procedures for mitigation. Aside from the negative externalities, controlled flame generated carbon nanoparticle synthesis has the potential to increase efficiency and reduce cost for a variety of industrial and technical applications. The primary goal of any fundamental flame study is to relate experimental properties to the real world operating conditions of modern engines. Modern engines exhibit complex turbulence, flow strain, and fuel air mixing under high pressure. As such, the goal of this work was to investigate and analyze the effect of elevated pressure on the soot characteristics of hydrocarbon combustion over a wide range of flow strain and residence time.

A high pressure counterflow non-premixed burner was chosen as the experimental con-

figuration. Laser induced incandescence and particle image velocimetry were chosen to conduct measurements of soot characteristics and flow strain respectively. Thermophoretic soot sampling and transmission electron microscopy analysis was also utilized to provide insight into soot characteristics and morphology. The development of the absolute irradi- 
ance calibrated laser induced incandescence system was reported in detail including submodels for processing elastic light scattering data and laser and signal extinction effects.

An extensive analysis was conducted upon the development of the LII system applied to soot formation in the non-premixed counterflow flame geometry. A detailed uncertainty analysis was completed to identify key uncertain parameters dominating the uncertainty of LII measured soot quantities. Several improvements were suggested to reduce measured uncertainties including the use of a mixture averaged species thermal accommodation coefficient to best match local species concentrations. The preliminary effort reduced uncertainties related to thermal accommodation and identified a need in literature for a complete library of temperature dependent species thermal accommodation data. An additional uncertainty reduction strategy was proposed with a local absorption function fitting procedure. While serving to reduce uncertainty on the measurement of volume fraction, the strategy fixed the absorption function more in line with the local LII physics and added further insight into changes in local soot morphology.

Recommended improvements to LII measurement uncertainties were successful yet total measurement uncertainties remained significant. The final LII measurement uncertainties applicable to this study for the absorption function of soot $E(m)$, soot volume fraction $f_{v}$, primary particle diameter $d_{p}$, and number density $N$ were $26 \%, 30 \%, 44-50 \%$, and $82-91 \%$ respectively with the range for $d_{p}$ and $N$ given depending on a flame diluent gas of either helium or nitrogen. Focused efforts on only a few key variables namely the local temperature and thermal accommodation would reduce the uncertainty on $E(m), f_{v}, d_{p}$, and $N$ to approximately $18 \%, 21 \%, 26 \%$, and $48 \%$ respectively and represent an approximate floor to LII model uncertainty. Repeatability studies indicated that uncertainty due to repeatability was much less than model uncertainty on the order of $\sim 10-15 \%$ for soot volume fraction and primary particle size.

A detailed uncertainty analysis of particle image velocimetry measurements was also conducted. A Monte Carlo approach was employed to account for all additional mea- 
surement uncertainties while using linear least squares regression to determine flow strain. Standard error estimates for local strain rates at pressures from 1 atm to 14 atm varied between 8 and $25 \%$ with results at pressures of $20 \mathrm{~atm}$ and $30 \mathrm{~atm}$ around $30-65 \%$ due to issues of optical distortion and seed particle coagulation.

Experimental results were reported for TEM analysis of thermophoretically sampled soot. While not applicable to the majority of collected data, soot aggregate dimensions were determined from data at 8.45 atm where aggregation was observed. The results gave fractal dimensions $D_{f}=1.41$ and $k_{f}=2.9$ indicating densely packed aggregates where persistent particle surface growth was evident. Primary particle size and aggregate size distributions were also determined to exhibit a narrow lognormal distribution width. Finely ordered annealed soot structure was not prevalent in the soot samples collected indicating significant PAH surface growth at low temperature.

An extensive analysis of soot number density, volume fraction, and primary particle size was conducted from LII data of sooting counterflow flames over a range of strain rates and pressures. A sample of the data collected for particle temperature, soot volume fraction $f_{v}$, primary particle diameter $d_{p}$, and number density $N$ were reported with extensive effort committed to determined the effects of pressure and strain rate on soot nucleation and surface growth mechanisms. The elastic scattering submodel was also tested under conditions of observed soot aggregation. The results presented were in acceptable agreement with TEM data but suffered from issues of poor fitting and indefinite solution where significant improvement to the procedure should be explored. Finally, strain rates corresponding to sooting limits were reported. The results served to target the sensitivity of highly uncertain soot nucleation mechanisms.

With the development of the LII system described in this work and a completed dataset of soot properties collected over a range of strain rates and pressures, some key conclusions can be drawn.

- The absolute irradiance calibrated LII system offers an excellent method for collect- 
ing quantitative soot incandescence data in the counterflow geometry and requires no other external or inline calibrations. LII extracted profiles of soot incancescence even under relatively low soot loading and low signal to noise still result in well described profiles of peak particle temperature, primary particle size, soot volume fraction, and number density.

- Measurement uncertainties on LII extracted quantities are high and significantly larger than repeatability uncertainty indicating a need for additional improvements to uncertain LII model variables including the local gas temperature and thermal accommodation between a soot particle surface and the surrounding gas.

- An analysis of LII extracted soot properties of volume fraction $f_{v}$, primary particle diameter $d_{p}$, and number density $N$ indicates particle nucleation is the driver of total soot loading in the counterflow configuration and exhibits a clear temperature and pressure dependency. Additionally, soot surface growth as a function of temperature remains relatively constant with changes in pressure. This results in constant maximum particle size with changes in strain rate where particle residence time in the reaction layer remains relatively unchanged.

Applying the conclusions developed in this work to practical engine design is of paramount importance. Modern engines generally exhibit highly turbulent flows where local flamelets and flow strain conditions evolve rapidly over time. In an instant a rich mixing and reacting layer in an engine can in many ways be related to the same physics governing counterflow flames. The connection allows for an extension of the conclusions made in this study. For a rich mixing and reacting flow, increasing pressure past approximately 4 atm has the effect of staunching soot nucleation at higher temperatures. This indicates increasing combustion pressure could significantly reduce the total amount of soot produced in an engine. Lastly, increasing pressure indicates higher combustion temperatures could also be employed before soot nucleation is triggered. From thermodynamic first principles, 
increasing pressure and temperature are directly related to increased engine efficiency and energy density. Strictly concerning soot formation, the findings are encouraging for industry and indicate a scenario with the potential to improve efficiency while also reducing a key combustion pollutant.

Several avenues are suggested for future exploration and improvement to the measurement procedure developed in this study.

- A means of anchoring model and experimental data to remove temperature uncertainty in the counterflow configuration would significantly improve measurement uncertainty. A means of precisely measuring the gas temperature would also accomplish the same objective.

- A wide angle light scattering submodel and experimental configuration should be implemented for accurately measuring soot aggregate sizes.

- An instantaneous LII system could be developed using two intensified cameras. Incandescent signals at two wavelengths could be recorded with one camera using the proper splitting optics with the second camera recording a later signal of the incandescent signal decay for particle sizing. A third camera for recording wide angle elastic light scattering could also be applied.

- The measurement procedure developed in this study could be extended to other fuels and configurations including oxidation to target other soot mechanisms. 


\section{Bibliography}

[1] Energy Information Administration (US). Annual Energy Outlook 2011, With Projections to 2035, volume 0383. Energy Information Administration, 2011.

[2] I. Glassman and R. Yetter. Combustion. Elsevier Science, 2008.

[3] A. Seaton, W. MacNee, K. Donaldson, and D. Godden. Particulate air pollution and acute health effects. Lancet, 345(8943):176-8, January 1995.

[4] W. L. Chameides and M. Bergin. Climate change. Soot takes center stage. Science (New York, N.Y.), 297(5590):2214-5, September 2002.

[5] J. Fenger. Air pollution in the last 50 years-From local to global. Atmospheric Environment, 43(1):13-22, January 2009.

[6] H. Wang. Formation of nascent soot and other condensed-phase materials in flames. Proceedings of the Combustion Institute, 33(1):41-67, 2011.

[7] I. M. Kennedy. Models of soot formation and oxidation. Progress in Energy and Combustion Science, 23:95-132, 1997.

[8] Directive 2008/50/EC of the European Parliament and of the Council of 21 May 2008 on ambient air quality and cleaner air for Europe, 2008.

[9] P. Desgroux, X. Mercier, and K. A. Thomson. Study of the formation of soot and its precursors in flames using optical diagnostics. Proceedings of the Combustion Institute, 34(1):1713-1738, January 2013.

[10] D. X. Du, H. Wang, and C. K. Law. Soot Formation in Counterflow Ethylene Diffusion Flames from 1 to 2.5 Atmospheres. Combustion and flame, 270:264-270, 1998.

[11] D. Bento, K. A. Thomson, and Ö. L. Gülder. Soot formation and temperature field structure in laminar propane-air diffusion flames at elevated pressures. Combustion and Flame, 145(4):765-778, June 2006.

[12] M. S. Tsurikov, K. P. Geigle, V. Krüger, Y. Schneider-Kühnle, W. Stricker, R. Lückerath, R. Hadef, and M. Aigner. Laser-Based Investigation of Soot Formation in Laminar Premixed Flames At Atmospheric and Elevated Pressures. Combustion Science and Technology, 177(10):1835-1862, October 2005. 
[13] H. a. Michelsen, A. V. Tivanski, M. K. Gilles, L. H. van Poppel, M. a. Dansson, and P. R. Buseck. Particle formation from pulsed laser irradiation of soot aggregates studied with a scanning mobility particle sizer, a transmission electron microscope, and a scanning transmission x-ray microscope. Applied optics, 46(6):959-77, February 2007.

[14] R. L. Vander Wal, V. M. Bryg, and C.-H. Huang. Aircraft engine particulate matter: Macro- micro- and nanostructure by HRTEM and chemistry by XPS. Combustion and Flame, September 2013.

[15] M. P. Johnson, M. Hilton, D. R. Waterman, and J. D. Black. Development of techniques to characterize particulates emitted from gas turbine exhausts. Measurement Science and Technology, 1146, 2003.

[16] J. S. Kinsey. Characterization of emissions from commercial aircraft engines during the Aircraft Particle Emissions eXperiment (APEX) 1 to 3. Technical Report October, U.S. Environmental Protection Agency, Washington DC, 2009.

[17] M. Di Domenico, P. Gerlinger, and M. Aigner. Development and validation of a new soot formation model for gas turbine combustor simulations. Combustion and Flame, 157(2):246-258, February 2010.

[18] D. R. Snelling, G. J. Smallwood, R. A. Sawchuk, W. S. Neill, D. Gareau, W. L. Chippior, F. Liu, Ö. L. Gülder, and W. D. Bachalo. Particulate Matter Measurements in a Diesel Engine Exhaust by Laser-Induced Incandescence and the Standard Gravimetric Procedure. 1999.

[19] A. Kay and M. Grätzel. Low cost photovoltaic modules based on dye sensitized nanocrystalline titanium dioxide and carbon powder. Solar Energy Materials and Solar Cells, 44:99-117, 1996.

[20] R. Taylor, S. Coulombe, T. Otanicar, P. Phelan, A. Gunawan, W. Lv, G. Rosengarten, R. Prasher, and H. Tyagi. Small particles, big impacts: A review of the diverse applications of nanofluids. Journal of Applied Physics, 113(1):011301, 2013.

[21] C. Portet, G. Yushin, and Y. Gogotsi. Electrochemical performance of carbon onions, nanodiamonds, carbon black and multiwalled nanotubes in electrical double layer capacitors. Carbon, 45(13):2511-2518, November 2007.

[22] H. Richter and J. B. Howard. Formation of polycyclic aromatic hydrocarbons and their growth to soot-a review of chemical reaction pathways. Progress in Energy and Combustion Science, 26:565-608, 2000.

[23] K. A. Thomson, Ö. L. Gülder, E. Weckman, R. Fraser, G. J. Smallwood, and D. R. Snelling. Soot concentration and temperature measurements in co-annular, nonpremixed $\mathrm{CH} 4 /$ air laminar flames at pressures up to $4 \mathrm{MPa}$. Combustion and Flame, 140(3):222-232, February 2005. 
[24] M. Hofmann, B. F. Kock, T. Dreier, H. Jander, and C. Schulz. Laser-induced incandescence for soot-particle sizing at elevated pressure. Applied Physics B, 90(34):629-639, December 2007.

[25] J. Y. Hwang and S. H. Chung. Growth of Soot Particles in Counterflow Diffusion Flames of Ethylene. Combustion and Flame, 762:752-762, 2001.

[26] H. I. Joo and Ö. L. Gülder. Experimental study of soot and temperature field structure of laminar co-flow ethylene-air diffusion flames with nitrogen dilution at elevated pressures. Combustion and Flame, 158(3):416-422, March 2011.

[27] M. Hofmann. Laser-Induced Incandescence for Soot Diagnostics at High Pressures. PhD thesis, University of Heidelberg, April 2006.

[28] L. Figura and A. Gomez. Laminar counterflow steady diffusion flames under high pressure $(\mathrm{P}<=3 \mathrm{MPa})$ conditions. Combustion and Flame, 159(1):142-150, July 2011.

[29] A. E. Karatas and Ö. L. Gülder. Soot formation in high pressure laminar diffusion flames. Progress in Energy and Combustion Science, 38(6):818-845, December 2012.

[30] C. M. Sorensen. Light Scattering by Fractal Aggregates : A Review. Aerosol Science \& Technology, (June 2013):37-41, 2001.

[31] R. L. Vander Wal and K. J. Weiland. Laser-induced incandescence: Development and characterization towards a measurement of soot-volume fraction. Applied Physics B: Lasers and Optics, 59:445-452, 1994.

[32] K. T. Kang, J. Y. Hwang, S. H. Chung, and W. Lee. Soot Zone Structure and Sooting Limit in Diffusion Flames : Comparison of Counterflow and Co-Flow Flames. Combustion and Flame, 281:266-281, 1997.

[33] C. P. Leusden and N. Peters. Experimental and numerical analysis of the influence of oxygen on soot formation in laminar counterflow flames of acetylene. Proceedings of the Combustion Institute, 28(2):2619-2625, January 2000.

[34] K. A. Thomson, D. R. Snelling, G. J. Smallwood, and F. Liu. Laser induced incandescence measurements of soot volume fraction and effective particle size in a laminar co-annular non-premixed methane/air flame at pressures between 0.5-4.0 MPa. Applied Physics B, 83(3):469-475, April 2006.

[35] B. G. Sarnacki, G. Esposito, R. H. Krauss, and H. K. Chelliah. Extinction limits and associated uncertainties of nonpremixed counterflow flames of methane, ethylene, propylene and n-butane in air. Combustion and Flame, 159(3):1026-1043, November 2011. 
[36] R. L. Vander Wal, T. M. Ticich, and A. Brock Stephens. Can soot primary particle size be determined using laser-induced incandescence? Combustion and Flame, 116(1-2):291-296, January 1999.

[37] M. Mikofski, T. Williams, C. Shaddix, A. Fernandez-Pello, and L. Blevins. Structure of laminar sooting inverse diffusion flames. Combustion and Flame, 149(4):463478, June 2007.

[38] L. L. McCrain and W. L. Roberts. Measurements of the soot volume field in laminar diffusion flames at elevated pressures. Combustion and Flame, 140(1-2):60-69, January 2005.

[39] C. H. Kim, F. Xu, and G. M. Faeth. Soot surface growth and oxidation at pressures up to 8.0 atm in laminar nonpremixed and partially premixed flames. Combustion and Flame, 152(3):301-316, February 2008.

[40] T. Yelverton and W. L. Roberts. Soot surface temperature measurements in pure and diluted flames at atmospheric and elevated pressures. Experimental Thermal and Fluid Science, 33(1):17-22, October 2008.

[41] F. Xu, P. B. Sunderland, and G. M. Faeth. Soot formation in laminar premixed ethylene/air flames at atmospheric pressure. Combustion and Flame, 108(4):471493, 1997.

[42] F. Inal and S. M. Senkan. Effects of Equivalence Ratio on Species and Soot Concentrations in Premixed N-Heptane Flames. Combustion and Flame, 131:16-28, 2002.

[43] A. Ergut, R. J. Therrien, Y. a. Levendis, H. Richter, J. B. Howard, and J. B. Carlson. The effect of temperature on the soot onset chemistry in one-dimensional, atmospheric-pressure, premixed ethylbenzene flames. Combustion and Flame, 155(1-2):232-246, October 2008.

[44] H. I. Joo. Soot Formation in Non-Premixed Laminar Flames at Subcritical and Supercritical Pressures. 2010.

[45] K. Mcnesby, A. Miziolek, T. Nguyen, F. Delucia, R. Reedskaggs, and T. A. Litzinger. Experimental and computational studies of oxidizer and fuel side addition of ethanol to opposed flow air/ethylene flames. Combustion and Flame, 142(4):413427, September 2005.

[46] M. Yamamoto, S. Duan, and S. M. Senkan. The effect of strain rate on polycyclic aromatic hydrocarbon (PAH) formation in acetylene diffusion flames. Combustion and Flame, 151(3):532-541, November 2007.

[47] K. C. Lin and G. M. Faeth. Effects of Hydrodynamics on Soot Formation in Laminar Opposed-Jet Diffusion Flames. Journal of Propulsion and Power, 12(4):691-698, 1996. 
[48] A. D’anna, M. Commodo, M. Sirignano, P. Minutolo, and R. Pagliara. Particle formation in opposed-flow diffusion flames of ethylene: An experimental and numerical study. Proceedings of the Combustion Institute, 32(1):793-801, 2009.

[49] C. J. Sung, B. Li, H. Wang, and C. K. Law. Structure and sooting limits in counterflow methane/air and propane/air diffusion flames from 1 to 5 atmospheres. Symposium (International) on Combustion, pages 1523-1529, 1998.

[50] R. V. Ravikrishna, S. V. Naik, C. S. Cooper, and N. M. Laurendeau. Quantitative laser-induced fluorescence measurements and modeling of nitric oxide in highpressure (6-15atm) counter-flow diffusion flames. Combustion Science and Technology, 176, 2004.

[51] R. W. Weeks and W. W. Duley. Aerosol-particle sizes from light emission during excitation by TEA CO2 laser pulses. Journal of Applied Physics, 45(10):4661, 1974.

[52] A. C. Eckbreth. Effects of laser-modulated particulate incandescence on Raman scattering diagnostics. Journal of Applied Physics, 48(11):4473, 1977.

[53] L a Melton. Soot diagnostics based on laser heating. Applied optics, 23(13):2201, July 1984.

[54] J. E. Dec, A. O. zur Loye, and D. L. Siebers. Soot Distribution in a D.I. Diesel Engine Using 2-D Laser-Induced Incandescence Imaging. SAE Technical Paper 910224, 1991.

[55] P. Roth and A. Filippov. In Situ Ultrafine Particle Sizing By A Combination of Pulsed Laser Heatup and Particle Thermal Emission. Science, 27(1):95-104, 1996.

[56] S. Will, S. Schraml, and A. Leipertz. Two-dimensional soot-particle sizing by timeresolved laser-induced incandescence. Optics letters, 20(22):2342, November 1995.

[57] S. Dankers and A. Leipertz. Determination of primary particle size distributions from time-resolved laser-induced incandescence measurements. Applied Optics, 43(18):3726-3731, 2004.

[58] B. Quay, T. Lee, T. Ni, and R. Santoro. Spatially Resolved Measurements of Soot Volume Fraction Using Laser-Induced Incandescence. Combustion and Flame, 97:384-392, 1994.

[59] T. Ni, J. a. Pinson, S. Gupta, and R. Santoro. Two-dimensional imaging of soot volume fraction by the use of laser-induced incandescence. Applied optics, 34(30):7083-91, October 1995.

[60] M. Hofmann, W. G. Bessler, C. Schulz, and H. Jander. Laser-Induced Incandescence for Soot Diagnostics at High Pressures. Applied Optics, 42(12):2052-2062, 2003. 
[61] D. R. Snelling, G. J. Smallwood, F. Liu, Ö. L. Gülder, and W. D. Bachalo. A calibration-independent laser-induced incandescence technique for soot measurement by detecting absolute light intensity. Applied optics, 44(31):6773-85, November 2005 .

[62] M. Hazelgrove, D. Levick, and N. Le. Design of an Apparatus to Study Soot Formation in High-Pressure Gas Turbines. B.s. thesis, University of Virginia, 2010.

[63] J. M. Bergthorson, K. Sone, T. W. Mattner, P. E. Dimotakis, D. G. Goodwin, and D. I. Meiron. Impinging laminar jets at moderate Reynolds numbers and separation distances. Physical review. E, Statistical, nonlinear, and soft matter physics, 72(6 Pt 2):066307, December 2005.

[64] D. Hoffman. Design, Prototyping, and Fabrication of an Optimal Counterflow Burner Nozzle. B.s. thesis, University of Virginia, 2012.

[65] R. B. King. Relative Transition Probabilities of the Swan Bands of Carbon. Astrophysical Journal, 108:429, 1948.

[66] H. K. Chelliah, C. K. Law, T. Ueda, M. D. Smooke, and F. A. Williams. An experimental and theoretical investigation of the dilution, pressure and flow-field effects on the extinction condition of methane-air-nitrogen diffusion flames. Symposium (International) on Combustion, 23(1):503-511, 1991.

[67] H. Wang, E. Dames, B. Sirjean, D. A. Sheen, R. Tangko, A. Violi, J. Y. W. Lai, F. N. Egolfopoulos, D. F. Davidson, R. K. Hanson, C. T. Bowman, C. K. Law, W. Tsang, N. P. Cernansky, D. L. Miller, and R. P. Lindstedt. A high-temperature chemical kinetic model of n-alkane (up to n-dodecane), cyclohexane, and methyl-, ethyl-, npropyl and n-butyl-cyclohexane oxidation at high temperatures (jetsurf 2.0). Technical report, Combustion Kinetics Laboratory, University of Southern California, 2011.

[68] H. Wang and M. B. Colket. Validation of SERDP Model (ver 0.1) of PAH formation in Ethylene Combustion. Technical report, SERDP, 2011.

[69] M. D. Smooke, J. Crump, K. Seshadri, and V. Giovangigli. Comparison between experimental measurements and numerical calculations of the structure of counterflow, diluted, methane-air, premixed flames. Symposium (International) on Combustion, 23(1):463-470, 1991.

[70] H. a. Michelsen, F. Liu, B. F. Kock, H. Bladh, a. Boiarciuc, M. Charwath, T. Dreier, R. Hadef, M. Hofmann, J. Reimann, S. Will, P.-E. Bengtsson, H. Bockhorn, F. Foucher, K. P. Geigle, C. Mounaïm-Rousselle, C. Schulz, R. Stirn, B. Tribalet, and R. Suntz. Modeling laser-induced incandescence of soot: a summary and comparison of LII models. Applied Physics B, 87(3):503-521, April 2007.

[71] C. Schulz, B. F. Kock, M. Hofmann, H. a. Michelsen, S. Will, B. Bougie, R. Suntz, and G. J. Smallwood. Laser-induced incandescence: recent trends and current questions. Applied Physics B, 83(3):333-354, May 2006. 
[72] T. Bond and R. Bergstrom. Light Absorption by Carbonaceous Particles: An Investigative Review. Aerosol Science \& Technology, 40(1):27-67, January 2006.

[73] F. S. Sherman. A Survey of Experimental Results and Methods for the Transition Regime of Rarefied Gas Dynamics. In J. A. Lauermann, editor, Rarefied Gas Dynamics, Volume 2, page 228. Academic Press, New York, 1963.

[74] F. Liu, K. J. Daun, D. R. Snelling, and G. J. Smallwood. Heat conduction from a spherical nano-particle: status of modeling heat conduction in laser-induced incandescence. Applied Physics B, 83(3):355-382, April 2006.

[75] A. M. Brasil, T. L. Farias, and M. G. Carvalho. a Recipe for Image Characterization of Fractal-Like Aggregates. Journal of Aerosol Science, 30(10):1379-1389, December 1999.

[76] K. J. Daun. Effect of Selective Accommodation on Soot Aggregate Shielding in Time-Resolved Laser-Induced Incandescence Experiments. Journal of Heat Transfer, 132(9):091202, 2010.

[77] A. Filippov, M. Zurita, and D. E. Rosner. Fractal-like Aggregates: Relation between Morphology and Physical Properties. Journal of colloid and interface science, 229(1):261-273, September 2000.

[78] F. Liu, M. Yang, F. a. Hill, D. R. Snelling, and G. J. Smallwood. Influence of polydisperse distributions of both primary particle and aggregate size on soot temperature in low-fluence LII. Applied Physics B, 83(3):383-395, April 2006.

[79] G. Esposito and H. K. Chelliah. Skeletal reaction models based on principal component analysis: Application to ethylene-air ignition, propagation, and extinction phenomena. Combustion and Flame, 158(3):477-489, March 2011.

[80] D. A. Sheen and H. Wang. Combustion kinetic modeling using multispecies time histories in shock-tube oxidation of heptane. Combustion and Flame, 158(4):645656, April 2011.

[81] R. J. Kee, F. M. Rupley, and J. A. Miller. CHEMKIN-II: A FORTRAN Chemical Kinetics Package for the Analysis of Gas-Phase Chemical Kinetics. Technical report, 1989.

[82] R. J. Kee, G. Dixon-lewis, J. Warnatz, M. E. Coltrin, and J. A. Miller. A Fortran Computer Code Package For The Evaluation Of Gas-Phase, Multicomponent Transport Properties. Technical report, 1986.

[83] H. a. Michelsen. Understanding and predicting the temporal response of laserinduced incandescence from carbonaceous particles. The Journal of Chemical Physics, 118(15):7012, 2003.

[84] L. E. Fried and W. M. Howard. Explicit Gibbs free energy equation of state applied to the carbon phase diagram. Physical Review B, 61(13):8734-8743, 2000. 
[85] H. Leider, O. Krikorian, and D. Young. Thermodynamic properties of carbon up to the critical point. Carbon, 11(5):555-563, October 1973.

[86] B. J. McCoy and C. Y. Cha. Transport phenomena in the rarefied gas transition regime. Chemical Engineering Science, 29(2):381-388, February 1974.

[87] B. F. Kock, C. Kayan, J. Knipping, H. R. Orthner, and P. Roth. Comparison of LII and TEM sizing during synthesis of iron particle chains. Proceedings of the Combustion Institute, 30(1):1689-1697, January 2005.

[88] R. Stirn, T. G. Baquet, S. Kanjarkar, W. Meier, K. P. Geigle, H. H. Grotheer, C. Wahl, and M. Aigner. Comparison of Particle Size Measurements with LaserInduced Incandescence, Mass Spectroscopy, and Scanning Mobility Particle Sizing in a Laminar Premixed Ethylene/Air Flame. Combustion Science and Technology, 181(2):329-349, January 2009.

[89] J. Reimann, S. A. Kuhlmann, and S. Will. 2D aggregate sizing by combining laserinduced incandescence (LII) and elastic light scattering (ELS). Applied Physics B, 96(4):583-592, May 2009.

[90] C. F. Bohren and D. R. Huffman. Absorption and scattering of light by small particles. Wiley science paperback series. Wiley, 1983.

[91] O. Koylu, G. M. Faeth, T. L. Farias, and M. G. Carvalho. Fractal and Projected Structure Properties of Soot Aggregates. Combustion and Flame, 633(94):621-633, 1995.

[92] M. Sneep and W. Ubachs. Direct measurement of the Rayleigh scattering cross section in various gases. Journal of Quantitative Spectroscopy and Radiative Transfer, 92(3):293-310, May 2005.

[93] M. Y. Choi and K. A. Jensen. Calibration and correction of laser-induced incandescence for soot volume fraction measurements. Combustion and Flame, 112(4):485491, 1998.

[94] J. Lee, I. Altman, and M. Y. Choi. Design of thermophoretic probe for precise particle sampling. Journal of Aerosol Science, 39(5):418-431, May 2008.

[95] R. A. Dobbins and C. M. Megaridis. Morphology of flame-generated soot as determined by thermophoretic sampling. Langmuir, (11):254-259, 1987.

[96] X. L. Zhu and J. P. Gore. Radiation effects on combustion and pollutant emissions of high-pressure opposed flow methane/air diffusion flames. Combustion and Flame, 141(1-2):118-130, April 2005.

[97] K. J. Daun, G. J. Smallwood, and F. Liu. Investigation of Thermal Accommodation Coefficients in Time-Resolved Laser-Induced Incandescence. Journal of Heat Transfer, 130(12):121201, 2008. 
[98] K. J. Daun. Thermal accommodation coefficients between polyatomic gas molecules and soot in laser-induced incandescence experiments. International Journal of Heat and Mass Transfer, 52(21-22):5081-5089, October 2009.

[99] J. Häger, D. Glatzer, H. Kuze, M. Fink, and H. Walther. Rotationally excited NO molecules incident on a graphite surface: molecular rotation and translation after scattering. Surface Science, 374(1-3):181-190, March 1997.

[100] O. Leroy, J. Perrin, J. Jolly, M. Péalat, and M. Lefebvre. Thermal accommodation of a gas on a surface and heat transfer in CVD and PECVD experiments. Journal of Physics D: Applied Physics, 30(4):499-509, February 1997.

[101] D. R. Snelling, F. Liu, G. J. Smallwood, and Ö. L. Gülder. Determination of the soot absorption function and thermal accommodation coefficient using low-fluence LII in a laminar coflow ethylene diffusion flame. Combustion and Flame, 136(1-2):180190, January 2004.

[102] S. A. Kuhlmann, J. Reimann, and S. Will. On heat conduction between laser-heated nanoparticles and a surrounding gas. Journal of Aerosol Science, 37(12):1696-1716, December 2006.

[103] M. W. Chase. NIST-JANAF thermochemical tables. 1998.

[104] F. Liu, B. Stagg, D. R. Snelling, and G. J. Smallwood. Effects of primary soot particle size distribution on the temperature of soot particles heated by a nanosecond pulsed laser in an atmospheric laminar diffusion flame. International Journal of Heat and Mass Transfer, 49(3-4):777-788, February 2006.

[105] K. J. Daun, G. J. Smallwood, and F. Liu. Molecular dynamics simulations of translational thermal accommodation coefficients for time-resolved LII. Applied Physics B, 94(1):39-49, November 2008.

[106] S. C. Saxena and R. K. Joshi. Thermal accommodation and adsorption coefficients of gases. Hemisphere Publishing Corporation, 1989.

[107] A. M. Kamat, A. C. T. van Duin, and A. Yakovlev. Molecular dynamics simulations of laser-induced incandescence of soot using an extended ReaxFF reactive force field. The journal of physical chemistry. A, 114(48):12561-72, December 2010.

[108] V. Kovalev, A. Yakunchikov, and F. Li. Tangential momentum and thermal accommodation coefficients for hydrogen molecules on graphite surface. Acta Astronautica, 69(7-8):744-746, September 2011.

[109] W. Watt. Carbon work at the royal aircraft establishment. Carbon, 10(2):121-143, April 1972.

[110] N. Herlin, M. Pealat, M. Lefebvre, P. Alnot, and J. Perrin. Rotational energy transfer on a hot surface in a low-pressure flow studied by CARS. Surface Science, 258(13):381-388, 1991. 
[111] G. M. Rosenblatt, R. S. Lemons, and C. W. Draper. Thermal accommodation coefficients by high speed vibration of solid samples. IV. Determination of translational and internal energy accommodation coefficients. The Journal of Chemical Physics, 67(3):1099, 1977.

[112] M. E. Thomas. Temperature dependence of the complex index of refraction. Handbook of Optical constants of Solids II, pages 177-201, 1991.

[113] S. C. Lee and C. L. Tien. Optical constants of soot in hydrocarbon flames. Symposium (International) on Combustion, 18(1):1159-1166, 1981.

[114] T. T. Charalampopoulos, H. Chang, and B. Stagg. The effects of temperature and composition on the complex refractive index of flame soot. Fuel, 68:1173-1179, 1989.

[115] S. di Stasio and P. Massoli. Influence of the soot property uncertainties in temperature and volume-fraction measurements by two-colour pyrometry. Measurement Science and Technology, 5:1453-1465, 1999.

[116] H. a. Michelsen, P. Schrader, and F. Goulay. Wavelength and temperature dependences of the absorption and scattering cross sections of soot. Carbon, 48(8):21752191, July 2010.

[117] M. Bruna and S. Borini. Optical constants of graphene layers in the visible range. Applied Physics Letters, 94(3):031901, 2009.

[118] a. V. Eremin, E. V. Gurentsov, E. Popova, and K. Priemchenko. Size dependence of complex refractive index function of growing nanoparticles. Applied Physics B, 104(2):285-295, February 2011.

[119] S. Wang, J. Mao, and G. Liu. Uncertainty of the PIV testing results for gas flow field caused by tracer particles and seeding method. AIP Conference Proceedings, (May 2009):148-155, 2007.

[120] M. Samimy and S. K. Lele. Motion of particles with inertia in a compressible free shear layer. Physics of Fluids, 3:1915-1923, August 1991.

[121] Carroll Croarkin and Paul Tobias, editors. NIST/SEMATECH e-Handbook of Statistical Methods. 2012.

[122] T. P. Ryan. Modern Engineering Statistics. Wiley, 2007.

[123] J. Neter and W. Wasserman. Applied Linear Statistical Models. The Irwin series in statistics. WCB/McGraw-Hill, 1996.

[124] F. Liu, G. J. Smallwood, and D. R. Snelling. Effects of primary particle diameter and aggregate size distribution on the temperature of soot particles heated by pulsed lasers. Journal of Quantitative Spectroscopy and Radiative Transfer, 93(1-3):301312, June 2005. 
[125] C. M. Sorensen, J. Cai, and N. Lu. Light-scattering measurements of monomer size, monomers per aggregate, and fractal dimension for soot aggregates in flames. Applied optics, 31(30):6547-57, October 1992.

[126] S. De Iuliis, F. Cignoli, S. Benecchi, and G. Zizak. Determination of soot parameters by a two-angle scattering-extinction technique in an ethylene diffusion flame. Applied optics, 37(33):7865-74, November 1998.

[127] C. P. Leusden, C. Hasse, and N. Peters. Aggregate Formation in Sooting Counterflow Diffusion Flames. Proceedings of the Combustion Institute, 29:2383-2390, 2002.

[128] D. R. Snelling, O. Link, K. A. Thomson, and G. J. Smallwood. Measurement of soot morphology by integrated LII and elastic light scattering. Applied Physics B, pages 385-397, 2011.

[129] H. Oltmann, J. Reimann, and S. Will. Single-shot measurement of soot aggregate sizes by wide-angle light scattering (WALS). Applied Physics B, 106(1):171-183, October 2011.

[130] V. Krüger, C. Wahl, R. Hadef, K. P. Geigle, W. Stricker, and M. Aigner. Comparison of laser-induced incandescence method with scanning mobility particle sizer technique: the influence of probe sampling and laser heating on soot particle size distribution. Measurement Science and Technology, 16(7):1477-1486, July 2005.

[131] W. A. de Heer and D. Ugarte. Carbon onions produced by heat treatment of carbon soot and their relation to the $217.5 \mathrm{~nm}$ interstellar absorption feature. Chemical physics letters, 207(4), 1993.

[132] Y. Kimura, T. Sato, and C. Kaito. Production and structural characterization of carbon soot with narrow UV absorption feature. Carbon, 42(1):33-38, January 2004.

[133] M. Frenklach. Reaction mechanism of soot formation in flames. Physical Chemistry Chemical Physics, 4(11):2028-2037, May 2002.

[134] F. Goulay, P. Schrader, L. Nemes, M. a. Dansson, and H. a. Michelsen. Photochemical interferences for laser-induced incandescence of flame-generated soot. Proceedings of the Combustion Institute, 32(1):963-970, 2009.

[135] M. Hofmann, B. F. Kock, T. Dreier, H. Jander, and C. Schulz. Laser-induced incandescence for soot-particle sizing at elevated pressure. Applied Physics B, 90(34):629-639, December 2007.

[136] F. A. Williams. Combustion Theory: The Fundamental Theory of Chemically Reacting Flow Systems. Combustion science and engineering series. Perseus Books, 1985.

[137] L. Talbot, R. K. Cheng, R. W. Schefer, and D. R. Willis. Thermophoresis of Particles in a Heated Boundary Layer. Journal of Fluid Mechanics, 101:737, 1980. 
[138] F. Zheng. Thermophoresis of spherical and non-spherical particles: a review of theories and experiments. Advances in colloid and interface science, 97(1-3):25578, March 2002.

[139] I. Preciado. Study of Soot Deposition in Flames and Its Effect on Heat Transfer to Metal Surfaces. The University of Utah, 2009.

[140] A. Kazakov and M. Frenklach. Dynamic Modeling of Soot Particle Coagulation and Aggregation: Implementation With the Method of Moments and Application to High-Pressure Laminar Premixed Flames. Combustion and Flame, 114(3-4):484501, August 1998.

[141] S. H. Chung and A. Violi. Insights on the nanoparticle formation process in counterflow diffusion flames. Carbon, 45(12):2400-2410, October 2007.

[142] A. D’anna. Combustion Generated Fine Carbonaceous Particles. Karlsruhe University Press, Karlsruhe, 2009.

[143] T. S. Totton, A. J. Misquitta, and M. Kraft. A quantitative study of the clustering of polycyclic aromatic hydrocarbons at high temperatures. Physical chemistry chemical physics : PCCP, 14(12):4081-94, March 2012.

[144] M. Frenklach and J. Warnatz. Detailed Modeling Of PAH Profiles In A Sooting LowPressure Acetylene Flame. Combustion Science and Technology, 51(4-6):265-283, 1987.

[145] H. Pitsch. Detailed Kinetic Reaction Mechanism For Ignition and Oxidation of Alpha-Methylnaphthalene. Twenty-Sixth Symposium (International) on Combustion, pages 721-728, 1996.

[146] H. Wang, D. X. Du, C. J. Sung, and C. K. Law. Experiments and Numerical Simulation on Soot Formation in Opposed-Jet Ethylene Diffusion Flames. Twenty-Sixth Symposium (International) on Combustion, pages 2359-2368, 1996.

[147] M.R. J. Charest, C. P. T. Groth, and Ö. L. Gülder. A numerical study on the effects of pressure and gravity in laminar ethylene diffusion flames. Combustion and Flame, 158(10):1933-1945, October 2011.

[148] P. Mitchell and M. Frenklach. Particle aggregation with simultaneous surface growth. Physical Review E, 67(6):061407, June 2003.

[149] H. a. Michelsen, P. O. Witze, D. Kayes, and S. Hochgreb. Time-resolved laserinduced incandescence of soot: the influence of experimental factors and microphysical mechanisms. Applied optics, 42(27):5577-90, September 2003.

[150] B. Yang and O. Koylu. Detailed soot field in a turbulent non-premixed ethylene/air flame from laser scattering and extinction experiments. Combustion and Flame, 141(1-2):55-65, April 2005. 